\title{
Towards proactive care for potentially frail older people in general practice : Development, feasibility, and effectiveness of the [G]OLD preventive home visitation programme
}

Citation for published version (APA):

Stijnen, M. M. N. (2015). Towards proactive care for potentially frail older people in general practice : Development, feasibility, and effectiveness of the [G]OLD preventive home visitation programme. [Doctoral Thesis, Maastricht University]. Datawyse / Universitaire Pers Maastricht. https://doi.org/10.26481/dis.20150212ms

Document status and date:

Published: 01/01/2015

DOI:

10.26481/dis.20150212ms

Document Version:

Publisher's PDF, also known as Version of record

Please check the document version of this publication:

- A submitted manuscript is the version of the article upon submission and before peer-review. There can be important differences between the submitted version and the official published version of record. People interested in the research are advised to contact the author for the final version of the publication, or visit the $\mathrm{DOI}$ to the publisher's website.

- The final author version and the galley proof are versions of the publication after peer review.

- The final published version features the final layout of the paper including the volume, issue and page numbers.

Link to publication

\footnotetext{
General rights rights.

- You may freely distribute the URL identifying the publication in the public portal. please follow below link for the End User Agreement:

www.umlib.nl/taverne-license

Take down policy

If you believe that this document breaches copyright please contact us at:

repository@maastrichtuniversity.nl

providing details and we will investigate your claim.
}

Copyright and moral rights for the publications made accessible in the public portal are retained by the authors and/or other copyright owners and it is a condition of accessing publications that users recognise and abide by the legal requirements associated with these

- Users may download and print one copy of any publication from the public portal for the purpose of private study or research.

- You may not further distribute the material or use it for any profit-making activity or commercial gain

If the publication is distributed under the terms of Article $25 \mathrm{fa}$ of the Dutch Copyright Act, indicated by the "Taverne" license above, 


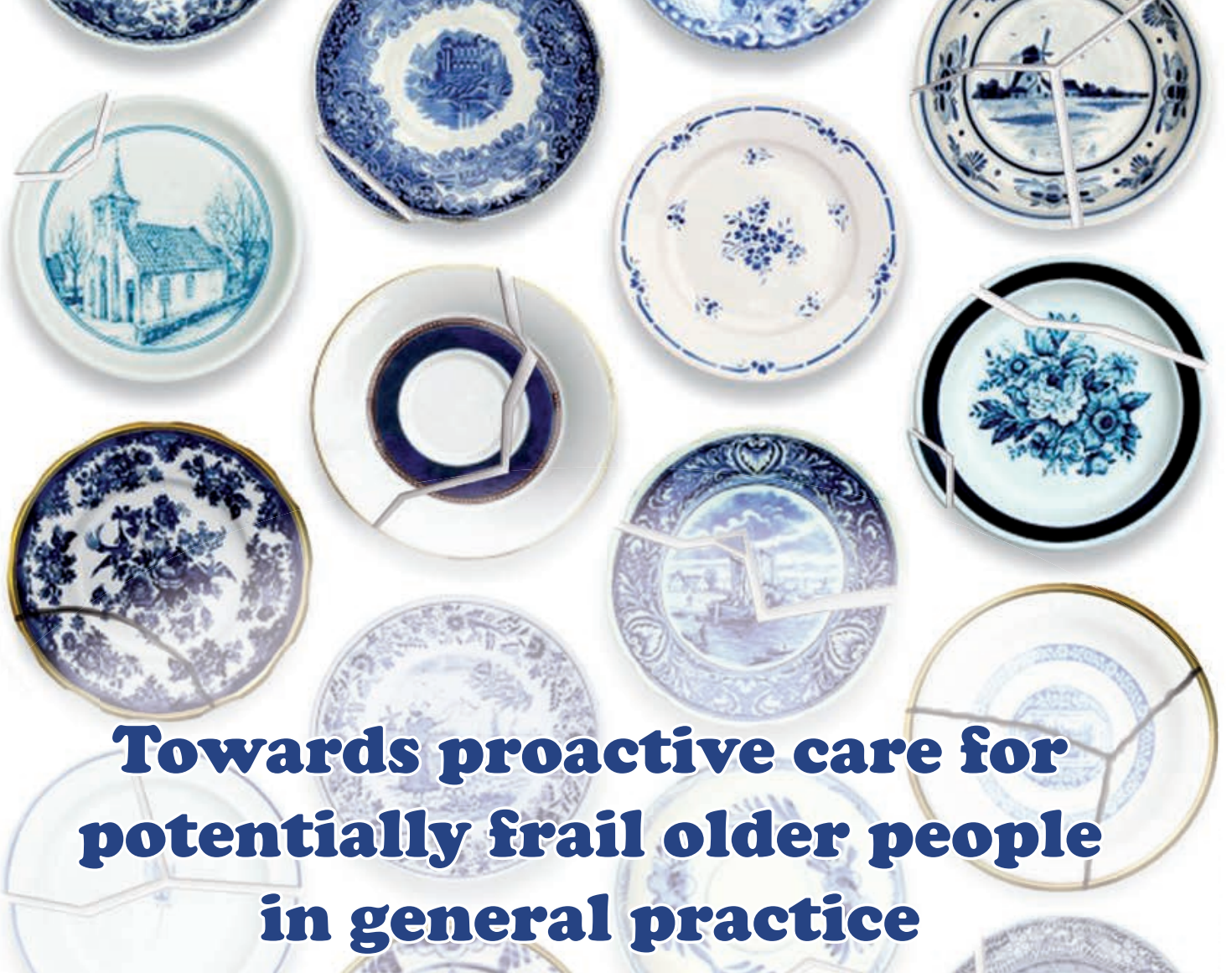

Development, feasibility, and effectiveness of the [G]OLD preventive home visitation programme
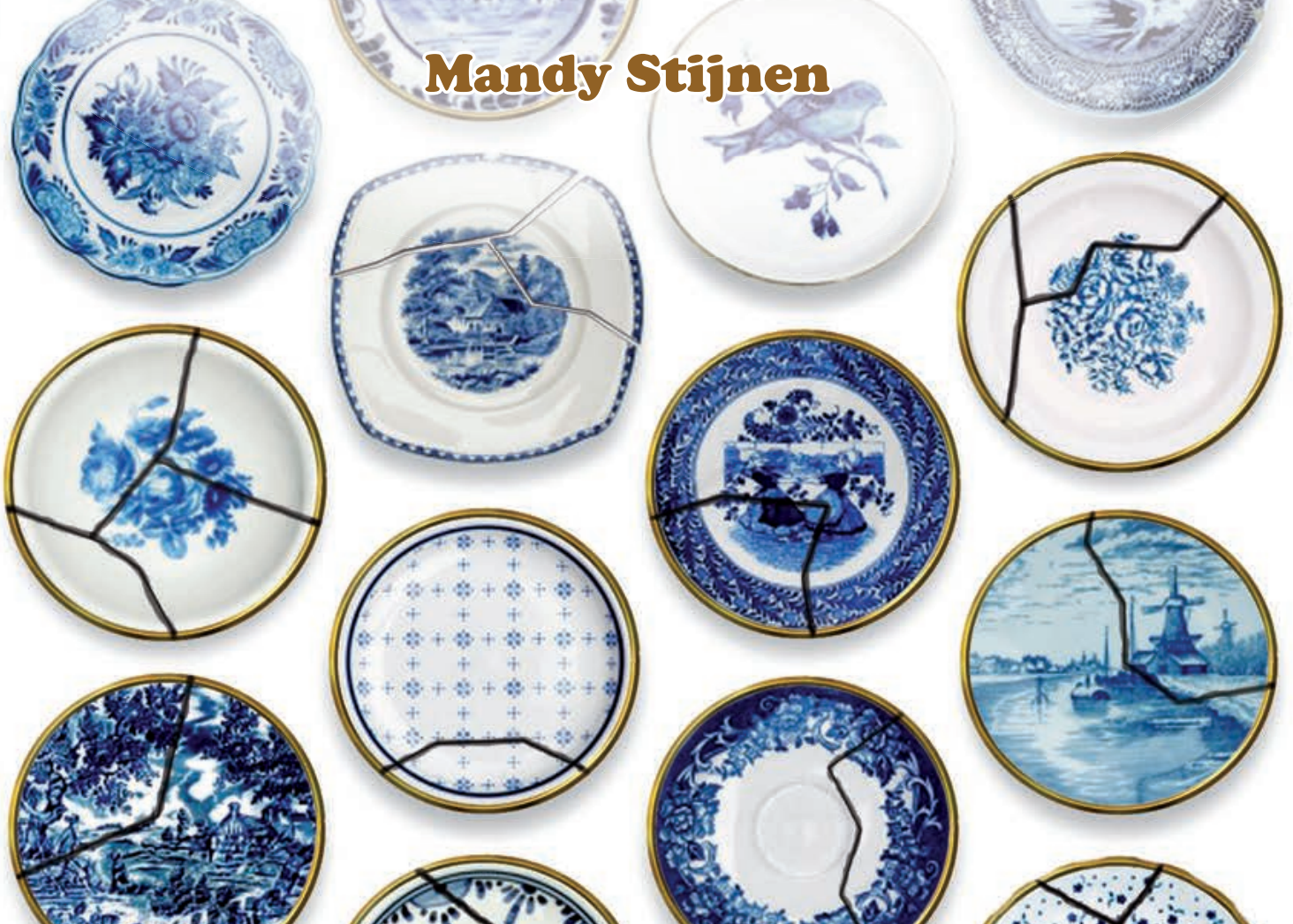


\section{Colophon}

Production: Datawyse | Universitaire Pers Maastricht Cover design: Deborah Stijnen

(c) Copyright Mandy Stijnen, Maastricht 2015

ISBN 9789461593924

The research presented in this thesis was conducted at the School for Public Health and Primary Care (CAPHRI), Department of Family Medicine, Maastricht University. CAPHRI participates in the Netherlands School of Primary Care Research ( $\mathrm{CaRe}$ ), acknowledged by the Royal Dutch Academy of Science (KNAW). CAPHRI was classified as 'excellent' by the external evaluation committee of leading international experts that reviewed CAPHRI in December 2010.

The studies presented in this thesis were funded by the Netherlands Organisation for Health Research and Development ('ZonMw', grant number 311070303), the Hague, the Netherlands, as part of The National Care for the Elderly Programme. 


\title{
Towards proactive care for potentially frail older people in general practice
}

\author{
Development, feasibility, and effectiveness of the \\ [G]OLD preventive home visitation programme
}

\author{
PROEFSCHRIFT \\ ter verkrijging van de graad van doctor aan de Universiteit Maastricht, \\ op gezag van de Rector Magnificus, Prof. dr. L.L.G. Soete, \\ volgens het besluit van het College van Decanen, \\ in het openbaar te verdedigen, \\ op donderdag 12 februari 2015 om 12.00 uur
}

door

Mandy Mathilda Nicolaas Stijnen

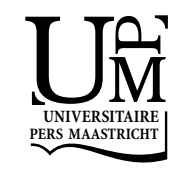




\section{Promotores}

Prof. dr. H.J.M. Vrijhoef (Tilburg University)

Prof. dr. ir. M.W.J. Jansen

\section{Copromotor}

Dr. I.G.P. Duimel-Peeters

\section{Beoordelingscommissie}

Prof. dr. J.F.M. Metsemakers (voorzitter)

Prof. dr. R. Huijsman (Erasmus Universiteit Rotterdam)

Prof. dr. G.I.J.M. Kempen

Prof dr. K.G. Luijkx (Tilburg University)

Prof. dr. G.D.E.M. van der Weijden 


\section{Contents}

$\begin{array}{lll}\text { Chapter } 1 \text { General introduction } & 7\end{array}$

Chapter 2 Development of a home visitation programme for the early detection of health problems in potentially frail community-dwelling older people by general practices

Chapter 3 Process evaluation plan of a patient-centred home visitation program for potentially frail community-dwelling older people in general practice

Chapter 4 Nurse-led home visitation programme to improve health-related quality of life and reduce disability among potentially frail community-dwelling older people in general practice: a theorybased process evaluation

Chapter 5 Formulation of and adherence to a care plan for potentially frail community-dwelling older people by practice nurses in the Netherlands

Chapter 6 Early detection of health problems in potentially frail communitydwelling older people by general practices - project [G]OLD: design of a longitudinal, quasi-experimental study

Chapter 7 Effectiveness of a nurse-led home visitation programme on healthrelated quality of life and disability among potentially frail community-dwelling older people in primary care: a longitudinal, quasi-experimental study

Chapter 8 Detected health and well-being problems following comprehensive geriatric assessment during a home visit among community-dwelling older people: who benefits most?

Chapter 9 General discussion

Valorisation

Summary

Samenvatting

Dankwoord

Curriculum Vitae

Publication list 



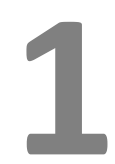

General introduction 



\section{INTRODUCTION}

As we grow old, we all hope to spend the last years of our lives as healthy and independently as possible in our own homes. Yet, with the rapid ageing of our population and increasing prevalence of chronic diseases, a growing number of older people is considered vulnerable. They face complex health and well-being problems at a certain point in their lives which restrict the performance of meaningful daily activities and threaten their ability to maintain independent living. Recent figures derived from a systematic review show that more than half of the population aged 60 years and older is confronted with a complex interplay of multiple, co-occurring health problems and/or chronic conditions, ${ }^{1}$ with prevalence numbers rising up to $71.8 \%$ in the general population aged 75 years old. ${ }^{2}$ For the older people concerned, the consequences are considerable, as they are at increased risk to suffer from, among others, poor healthrelated quality of life and disability. ${ }^{1}$ Healthcare systems are thus challenged with organising efficient and effective care to adequately respond to the emerging needs of the ageing population. ${ }^{3}$ It stands to reason that healthy ageing is therefore one of the core themes of national and international public health policy and research. ${ }^{4,5}$

Different concepts have been used interchangeably to denote the vulnerable subset of the older population requiring enhanced care, namely comorbidity, disability, or frailty. ${ }^{6}$ Although all three may co-occur in one person, they should be explicitly distinguished from one another. That is, comorbidity is a risk factor for frailty and disability is an outcome of frailty, and each confers its own care needs in older people. ${ }^{6,7}$ In achieving healthy ageing, functional decline and disability have been increasingly designated as important targets for prevention besides preventing or postponing (chronic) diseases. ${ }^{8,9}$ Since frailty is a precursor of disability, it seems worthwhile to concentrate preventive initiatives on frail older people. The focus of this dissertation is on a preventive home visitation programme for potentially frail community-dwelling older people ( $\geq 75$ years) within general practices. This chapter will further elaborate on the challenges imposed by population ageing in terms of frailty and disability, present the changes needed in our healthcare system to adequately care for older people, explore the appropriateness of the primary care setting for improving care for older people, summarise current evidence regarding preventive home visitation programmes, and identify gaps in the literature that serve as a starting point for the work presented in this dissertation. 


\section{POPULATION AGEING, FRAILTY AND DISABILITY}

Declining birth rates and increasing longevity have caused a rise in the number of older people who live into old age. Global projections reveal that population ageing will continue throughout the $21^{\text {st }}$ century and even at an accelerating pace in the coming decades. ${ }^{10}$ In the Netherlands, the proportion of people aged 65 years and older will increase from $15 \%$ in 2009 to nearly $26 \%$ in $2040 .{ }^{11}$ Not only will there be a large proportion of older people, but they also live longer lives. It is expected that more than $70 \%$ of the Dutch population aged 65 years in 2010 will reach the age of 80 years by 2025. Opposing viewpoints exist whether these substantial gains in life expectancy are accompanied by a postponement of disability and hence more years in good health. ${ }^{3,12}$ Disability is most commonly defined as dependency in carrying out daily activities essential to living independently at home, such as household tasks and self-care, as well as activities of importance for an older person's quality of life. ${ }^{6}$ It seems that in most European countries, rates of severe disability have declined over the past decades at the expense of a rise in mild disability and functional impairment. ${ }^{3}$

In addition, a growing proportion of older adults is considered frail. Frailty is often described as a state of vulnerability to sudden adverse health outcomes caused by minor stressor events as result of the accumulation of age-related and/or diseaseassociated deteriorations in physiological systems. ${ }^{6,13,14}$ Despite the emergent recognition of frailty as an important concept for clinical practice, there is a lack of agreement regarding its exact definition. ${ }^{15}$ Over the years, various conceptual and operational definitions of frailty have been proposed. ${ }^{15,16}$ In the frailty literature, two models for understanding and assessing frailty prevail: the frailty phenotype model ${ }^{7}$ and the cumulative deficit model. ${ }^{17}$ According to the frailty phenotype, five factors should be considered, that is unintentional weight loss, weak grip strength, poor endurance and energy, slow gait speed, and low physical activity level. People are considered frail when three or more of the above factors are present and those with one or two factors are judged to be pre-frail. ${ }^{7}$ The cumulative deficit model assumes that frailty is the result of the accumulative presence of deficits (e.g., symptoms, clinical signs, diseases, disabilities). As a result, a frailty index can be calculated that accounts for the number of deficits present out of all deficits considered. ${ }^{18,19}$ The more deficits are present, the more likely someone is frail. While the former model exclusively focuses on physical aspects of frailty, the latter also includes other aspects of health, such as medical conditions and health attitudes. In the work presented in this dissertation, frailty is considered a multifactorial concept resulting from the accumulation of problems related to physical, psychological, mental, and social functioning, lifestyle and medication use.

Prevalence rates of frailty in community-dwelling older people vary widely depending on the definition of frailty used. A systematic review of frailty prevalence rates 
by Collard and colleagues ${ }^{20}$ revealed that overall, $10.7 \%$ of community-dwelling older people aged 65 years and over were frail and $41.6 \%$ were pre-frail. Various studies have shown that frailty steadily increases with advancing age ${ }^{19,20}$ and is associated with adverse events, such as an increased risk of falls, hospitalisation, disability, morbidity, and mortality. ${ }^{7,19,21,22}$ Furthermore, frailty is a dynamic process which means that older people can move between different frailty states (i.e., non-frail, pre-frail, frail), with transitions to greater frailty being more common than transitions to lesser frailty. $^{23}$

\section{TOWARDS PATIENT-CENTRED, COHERENT, AND PROACTIVE CARE}

Our healthcare systems are not yet prepared to adequately respond to the changing needs of the fastest growing segment of our population. Up to now, healthcare systems have been largely configured according to a disease-oriented approach in which the prevention, diagnosis and treatment of individual diseases is the main focus of attention of care professionals. This is evidenced by the various disease management programmes introduced in primary care to improve healthcare quality and reduce healthcare costs of chronic diseases, such as diabetes mellitus, chronic obstructive pulmonary disease, and coronary heart disease. ${ }^{24,25}$ Especially frail older people who present themselves with complex problems in multiple health and well-being domains would benefit from a more holistic approach to care delivery in which the patient instead of the disease is the central point of attention. Furthermore, care for older people is fragmented. ${ }^{26}$ Frail older adults experience complex problems that require actions from both formal and informal caregivers, which are often poorly coordinated. In overcoming fragmentation, coherent care and continuity of care are required to maintain independent living. ${ }^{27}$ Finally, since different frailty states exist, ${ }^{7,23}$ frail older people should be identified at an early stage to prevent unnecessary neediness and maintain or improve independent functioning. This directs the attention away from a reactive approach to care delivery towards proactive care. There is a growing recognition that the primary care setting, and particularly general practices, have the potential to deliver patient-centred, coherent, and proactive care in line with older people's health and well-being needs. ${ }^{28}$

\section{CARE FOR OLDER PEOPLE IN GENERAL PRACTICES}

General practitioners (GPs) are increasingly confronted with older people who present themselves with complex care needs. In the Netherlands, the GP is often the first pro- 
fessional whom older people will approach in case they experience any health complaints, caused by the gatekeeping position of Dutch GPs to specialist care. ${ }^{29}$ Approximately five out of six Dutch adults aged 75 years and older had contact with their GP at least once in $2012 .^{30}$ Care for older people poses challenges to GPs, among others due to their vulnerability to rapid declines in health status and communication problems (e.g., caused by hearing problems or cognitive impairment). ${ }^{31}$ The resulting workload for GPs is huge. In 2012, the average number of contacts with general practice was 9.3 contacts for Dutch older people between 75 and 84 years of age and 13.2 contacts for the over-85-year-olds, compared to an average of 4.2 contacts across all age groups. ${ }^{32}$ In order to adequately address older people's problems in multiple health domains, shifting of care from the GP to other professionals within general practice is advocated. ${ }^{33}$ In relieving their workload, Dutch GPs therefore increasingly delegate care to the practice nurse (PN). PNs are employed by the GP with the initial aim to complement the GP in chronic care management by offering screening, treatment, care and education to chronically ill patients. Using a community-based case management approach, PNs deliver proactive care according to a cyclical process of assessment, care planning, implementation, monitoring, and evaluation. ${ }^{34}$ As a result, they are familiar with a patient-centred, holistic approach in providing care.

Besides the growing proportion of frail older people in need of care and available expertise in general practice to identify and address health problems in frail older people, frailty is also considered an important concept for general practices due to their biopsychosocial focus, patient-centredness, and familiarity with preventive measures. ${ }^{26,35}$ Furthermore, general practices have access to a range of services related to health, well-being, and social care in the older person's neighbourhood. They are therefore in the unique position to organise and coordinate proactive care. Also the identification of potentially frail older people could be carried out in general practice, as the early stages of frailty are most commonly seen in community-dwelling older people. $^{28}$

\section{PREVENTIVE HOME VISITATION PROGRAMMES}

Numerous interventions have been developed that aim to prevent the onset and/or progression of disability in community-dwelling frail older people. ${ }^{36}$ In particular, a large body of research is devoted to preventive home visitation programmes. They are defined as visits to independently living older people comprising a multidimensional assessment of older people's health, well-being, and functional status, which leads to specific health-enhancing recommendations in agreement with the older person's needs, and follow-up contacts to address identified health problems and the recom- 
mendations made earlier. ${ }^{37}$ Over the past two decades, several systematic reviews have been conducted regarding the effectiveness of preventive home visits for older people, yet the results remain inconclusive. Whereas some conclude that preventive home visits produce favourable outcomes in older people, ${ }^{38,39}$ others found inconsistent or no evidence for the effectiveness of preventive home visits. ${ }^{40-43}$ Nevertheless, it is stated that preventive home visitation programmes may work (i.e., have health promoting effects), ${ }^{44,}{ }^{45}$ however that heterogeneity in findings is caused by discrepancies in intervention content, population characteristics, adherence to the intervention protocol and recommendations from professionals, and setting. ${ }^{37,41,46}$

The multifaceted nature and a lack of detailed descriptions of the content of preventive home visitation programmes hamper the disentanglement of the independent effects of programme components on patient outcomes. ${ }^{38}$ Nevertheless, certain elements have been identified that appear to be conducive to favourable outcomes in older people, including a comprehensive geriatric assessment (CGA). ${ }^{39}$ The CGA goes beyond a standard medical evaluation by evaluating an older person's functional status, physical health, cognition, mental health, and socio-environmental circumstances. ${ }^{47}$ Systematic reviews have shown modest beneficial effects of CGA on functional status, health-related quality of life, and mortality. ${ }^{48,49}$ Moreover, a CGA can identify unmet needs in older people when applied in the general practice setting, ${ }^{50,51}$ though routine CGA for the general population of older adults is subject of discussion. ${ }^{52}$ To date, it is still unclear which older people benefit most from the CGA as well as from preventive home visits in general. ${ }^{53}$ Nonetheless, by means of a CGA, multiple and potentially co-existing health and well-being problems and risks can be addressed which is proposed as the most promising approach for older people at low risk of adverse events. ${ }^{53}$ Therefore, the focus is on the detection of health and wellbeing problems in potentially frail older people.

In sum, there is still an ongoing debate concerning the effectiveness of preventive home visits and whether they should be part of regular care for older people. Although it has been advocated to embed preventive home visits into clinical practice ${ }^{41}$ and general practices seem to be the ideal setting for this, little is known regarding the effectiveness of preventive home visitation programmes for older people in general practices. In addition to studying the effectiveness, available knowledge in this field would greatly benefit from a clear program theory guiding intervention design, a detailed description of program components, and a comprehensive process evaluation to evaluate the implementation of the intervention in the complex and dynamic general practice setting. Finally, in prioritising older people for the CGA from a practical point of view, GPs should be advised which subgroups of potentially frail older people benefit most from an in-home CGA. 


\section{AIM AND OBJECTIVES}

The aim of the research described in this dissertation is to assess the feasibility and effectiveness of the [G]OLD ('Getting OLD the healthy way') home visitation programme for potentially frail community-dwelling older people (aged $\geq 75$ years) in general practices. In order to achieve this, the main objectives that guided the individual studies as described in this dissertation were as follows:

1. To evaluate the preliminary feasibility of the [G]OLD home visitation programme in general practices.

2. To investigate whether the [G]OLD home visitation programme was implemented as planned and the extent to which general practices successfully redesigned their care delivery from reactive, disease-oriented care towards proactive, patientoriented care.

3. To investigate the effectiveness of the [G]OLD home visitation programme on the primary outcomes health-related quality of life and disability, and the secondary outcome attitude towards ageing.

4. To examine which community-dwelling older people benefit most from the [G]OLD comprehensive geriatric assessment in terms of detected health and/or well-being problems.

Besides these objectives, the dissertation provides a detailed overview of the design of a comprehensive, theory-based process evaluation plan for the [G]OLD home visitation programme, as well as the design of a cluster quasi-experimental controlled trial to examine to effectiveness of the [G]OLD home visitation programme.

\section{OUTLINE OF THE DISSERTATION}

In line with the framework of the Medical Research Council for the development and evaluation of complex interventions to improve health, ${ }^{54}$ the initial step was to determine intervention content, followed by a pilot test among a purpose sample of general practices to obtain insight into the preliminary feasibility of the [G]OLD home visitation programme. The process of developing the [G]OLD home visitation programme and the [G]OLD comprehensive geriatric assessment instrument used during the home visit, and findings regarding the preliminary feasibility according to general practices is described in Chapter 2. The results from this pilot study were used to improve the [G]OLD home visitation programme to allow for delivery of the intervention as planned. At the same time, a comprehensive evaluation plan comprising the design and procedures for assessing both the large-scale feasibility and effectiveness of the [G]OLD home visitation programme was written. Relatively uncommon, but of sub- 
stantial importance in evaluating complex interventions is a comprehensive, preplanned process evaluation to obtain a thorough understanding whether the [G]OLD home visitation programme was implemented as planned in general practices. This goes beyond assessing feasibility as done in the pilot study by providing in-depth insight into fidelity and quality of implementation of the [G]OLD home visitation programme in general practices and identifying factors that explain the success or failure of the parallel trial. Chapter 3 clarifies the rationale for and the steps undertaken in developing a theory-based process evaluation plan and the findings of the process evaluation are described in Chapter 4. Both the pilot study and the process evaluation revealed that one of the elements of the [G]OLD home visitation programme, the formulation of a care and treatment plan, was not implemented as planned. Therefore, an additional mixed-model study was designed and conducted to obtain a more detailed understanding of practice nurses' actions and views regarding the formulation of and adherence to the care and treatment plan, which is the focus of Chapter 5. Chapter 6 is devoted to presenting the design of a cluster quasi-experimental controlled trial in 24 general practices to investigate the effectiveness of the [G]OLD home visitation programme on the primary outcome measures health-related quality of life and disability at 6,12 , and 18 months follow-up. The findings of the quasi-experimental trial are reported in Chapter 7. Besides this, from the GP's point of view it is also relevant to know which older people benefit most from the [G]OLD home visitation programme in terms of the number of health and well-being problems detected by means of the [G]OLD-instrument. Chapter 8 explores this issue by differentiating between random selection of older people versus purposeful selection based on the GP's opinion whether certain pre-determined criteria are present in an older person that are indicative of experiencing more health and/or well-being problems now or at a near point in the future. Finally, Chapter 9 summarises and critically reflects on the main findings, elaborates on methodological considerations of the work presented in this dissertation, and discusses the implications for further research and for practice. 


\section{REFERENCES}

1. Marengoni A, Angleman S, Melis R, Mangialasche F, Karp A, Garmen A, et al. Aging with multimorbidity: a systematic review of the literature. Ageing Res Rev 2011;10:430-439.

2. Fortin $M$, Stewart $M$, Poitras $M$, Almirall J, Maddocks $H$. A systematic review of prevalence studies on multimorbidity: toward a more uniform methodology. Ann Fam Med 2012;10:142-151.

3. Rechel B, Doyle Y, Grundy E, McKee M. How can health systems respond to population ageing? Copenhagen: World Health Organization, 2009.

4. Infeld DL, Whitelaw N. Policy initiatives to promote healthy aging. Clin Geriatr Med 2002;18:627-642.

5. The Swedish National Institute of Public Health. Healthy ageing. A challenge for Europe. Stockholm: The Swedish National Institute of Public Health, 2006 Contract No.: R 2006:29.

6. Fried LP, Ferrucci L, Darer J, Williamson JD, Anderson G. Untangling the concepts of disability, frailty, and comorbidity: implications for improved targeting and care. J Gerontol A Biol Sci Med Sci 2004;59:255-263.

7. Fried LP, Tangen CM, Walston J, Newman AB, Hirsch C, Gottdiener J, et al. Frailty in older adults: evidence for a phenotype. J Gerontol A Biol Sci Med Sci 2001;56:M146-M157.

8. Health Council of the Netherlands. Prevention in the elderly: focus on functioning in daily life. The Hague: Health Council of the Netherlands, 2009 Contract No.: Publication no. 2009/07.

9. Hébert R. Functional decline in old age. Can Med Assoc J 1997;157:1037-1045.

10. Lutz W, Sanderson W, Scherbov S. The coming acceleration of global population ageing. Nature 2008;451:716-719.

11. Planbureau voor de Leefomgeving. Regionale prognose 2009-2040: Vergrijzing en omslag van groei naar krimp [Regional prognosis 2009-2040: Population ageing and shift from growth to shrinkage]. Bilthoven: Planbureau voor de Leefomgeving, 2010.

12. Christensen K, Doblhammer G, Rau R, Vaupel JW. Ageing populations: the challenges ahead. Lancet 2009;374:1196-1208.

13. Clegg A, Young J, Iliffe S, Olde Rikkert M, Rockwood K. Frailty in elderly people. Lancet 2013;381:752762.

14. Slaets JPJ. Vulnerability in the elderly: frailty. Med Clin North Am 2006;90:593-601.

15. Abellan Van Kan G, Rolland Y, Bergman H, Morley JE, Kritchevsky SB, Vellas B. The I.A.N.A. task force on frailty assessment of older people in clinical practice. J Nutr Health Aging 2008;12:29-37.

16. Gobbens RJ, Luijkx KG, Wijnen-Sponselee MT, Schols JM. Toward a conceptual definition of frail community dwelling older people. Nurs Outlook 2010;58:76-86.

17. Mitnitski AB, Mogilner AJ, Rockwood K. Accumulation of deficits as a proxy measure of aging. Sci World 2001;1:323-336.

18. Rockwood K, Song X, MacKnight C, Bergman H, Hogan DB, McDowell I, et al. A global clinical measure of fitness and frailty in elderly people. Can Med Assoc J 2005;173:489-495.

19. Song X, Mitnitski A, Rockwood K. Prevalence and 10-year outcomes of frailty in older adults in relation to deficit accumulation. J Am Geriatr Soc 2010;58:681-687.

20. Collard RM, Boter H, Schoevers RA, Oude Voshaar RC. Prevalence of frailty in community-dwelling older persons: a systematic review. J Am Geriatr Soc 2012;60:1487-1492.

21. Klein BEK, Klein R, Knudtson MD, Lee KE. Frailty, morbidity and survival. Arch Gerontol Geriatr 2005;41:141-149.

22. Shamliyan T, Talleyc KMC, Ramakrishnana R, Kane RL. Association of frailty with survival: a systematic literature review. Ageing Res Rev 2012.

23. Gill TM, Gahbauer EA, Allore HG, Han L. Transitions between frailty states among community-living older persons. Arch Intern Med 2006;166:418-423. 
24. Dennis SM, Zwar N, Grifftiths R, Roland M, Hasan I, Davies GP, et al. Chronic disease management in primary care: from evidence to policy. Med J Aust 2008;188:S53-S56.

25. Elissen AMJ. Going beyond the 'grand mean'. Advancing disease management science and evidence [PhD-thesis]. Maastricht: Maastricht University; 2013.

26. De Lepeleire J, Iliffe S, Mann E, Degryse J. Frailty: an emerging concept for general practice. Br J Gen Pract 2009;59:e177-e182.

27. Hallberg IR, Kristensson J. Preventive home care of frail older people: a review of recent case management studies. J Clin Nurs 2004;13:112-120.

28. Boeckxstaens P, De Graaf P. Primary care and care for older persons: position paper of the European Forum for Primary Care. Qual Prim Care 2011;19:369-389.

29. Willems DL. Balancing rationalities: gatekeeping in health care. J Med Ethics 2001;27:25-29.

30. Central Bureau of Statistics. Medische contacten, ziekenhuisopname, medicijnen; persoonskenmerken [Medical contacts, hospital admissions, medication; characteristics of individuals] Den Haag/Heerlen: Statline; 2014 [cited 2014 May 20th]. Available from: http://statline.cbs.nl/StatWeb/publication/?DM=SLNL\&PA=81027NED\&D1=0-44,46-58\&D2=0-2,13\&D3=0\&D4=I\&HDR=G3,G2,G1\&STB=T\&VW=T.

31. Adams WL, Mcllvain HE, Lacy NL, Magsi H, Crabtree BF, Yenny SK, et al. Primary care for elderly people. Why do doctors find it so hard? Gerontologist 2002;42:835-842.

32. NIVEL. Contacten huisartsenpraktijk [Contacts with general practice] Utrecht: NIVEL; 2014 [cited 2014 June 8th]. Available from: http://www.nivel.nl/NZR/contacten-huisartsenpraktijk.

33. Junius-Walker U, Wrede J, Schleef T, Diederichs-Egidi H, Wiese B, Hummers-Pradier E, et al. What is important, what needs treating? How GPs perceive older patients' multiple health problems: a mixed method research study. BMC Res Notes 2012;5:443.

34. Iliffe S, Drennan V. Primary care for older people. Oxford: Oxford University Press; 2000.

35. Lacas A, Rockwood K. Frailty in primary care: a review of its conceptualization and implications for practice. BMC Med 2012;10:4.

36. Daniels R, Metzelthin S, Van Rossum E, De Witte L, Van den Heuvel W. Interventions to prevent disability in frail community-dwelling older persons: an overview. Eur J Ageing 2010;7:37-55.

37. Markle-Reid M, Browne G, Weir R, Gafni A, Roberts J, Henderson SR. The effectiveness and efficiency of home-based nursing health promotion for older people: a review of the literature. Med Care Res Rev 2006;63:531-569.

38. Elkan R, Kendrick D, Dewey M, Hewitt M, Robinson J, Blair M, et al. Effectiveness of home based support for older people: systematic review and meta-analysis. Br Med J 2001;323:719-725.

39. Stuck AE, Egger M, Hammer A, Minder CE, Beck JC. Home visits to prevent nursing home admission and functional decline in elderly people: systematic review and meta-regression analysis. J Am Med Assoc 2002;287:1022-1029.

40. Bouman A, Van Rossum E, Nelemans P, Kempen GIJM, Knipschild P. Effects of intensive home visiting programs for older people with poor health status: a systematic review. BMC Health Serv Res 2008;8:74.

41. Huss A, Stuck AE, Rubenstein LZ, Egger M, Clough-Gorr KM. Multidimensional preventive home visit programs for community-dwelling older adults: a systematic review and meta-analysis of randomized controlled trials. J Gerontol A Biol Sci Med Sci 2008;63A:298-307.

42. Mayo-Wilson E, Grant S, Burton J, Parsons A, Underhill K, Montgomery P. Preventive home visits for mortality, morbidity, and institutionalization in older adults: a systematic review and meta-analysis. PLoS ONE 2014;9:e89257.

43. Van Haastregt JCM, Diederiks JPM, Van Rossum E, De Witte LP, Crebolder HFJM. Effects of preventive home visits to elderly people living in the community: systematic review. Br Med J 2000;320:754-758.

44. Fagerström L, Wikblad A, Nilsson J. An integrative research review of preventive home visits among older people - is an individual health resource perspective a vision or a reality? Scand J Caring Sci 2008;23:558-568. 
45. Egger M. Commentary: when, where, and why do preventive home visits work? $\mathrm{Br}$ Med J 2001;323:724-725.

46. Rubenstein LZ, Stuck AE. Preventive home visits for older people: defining criteria for success. Age Ageing 2001;30:107-109.

47. Elsawy B, Higgins KE. The geriatric assessment. Am Fam Physician 2011;83:48-56.

48. Stuck AE, Siu AL, Wieland GD, Adams J, Rubenstein LZ. Comprehensive geriatric assessment: a metaanalysis of controlled trials. Lancet 1993;342:1032-1036.

49. Van Mulligen-Van de Belt ECM, Smalbrugge M, Depla MFIA. Bewijs voor effectiviteit van Comprehensive Geriatric Assessment in de thuissituatie nog mager: een literatuurreview [Limited evidence for in-home comprehensive geriatric assessment: a review of literature]. Tijdschr Gerontol Geriatr 2013;44:215-227.

50. Lucchetti G, Granero AL. Use of comprehensive geriatric assessment in general practice: results from the 'Senta Pua' project in Brazil. Eur J Gen Pract 2011;17:20-27.

51. Piccoliori G, Gerolimon E, Abholz H. Geriatric assessment in general practice using a screening instrument: is it worth the effort? Results of a South Tyrol Study. Age Ageing 2008;37:647-652.

52. Iliffe $S$, Orrell M. Identifying unmet health needs in older people: comprehensive screening is not the answer. Br J Gen Pract 2006;56:404-406.

53. Stuck AE, Kane RL. Whom do preventive home visits help? J Am Geriatr Soc 2008;56:561-563.

54. Medical Research Council. Developing and evaluating complex interventions: new guidance. London: MRC, 2008. 


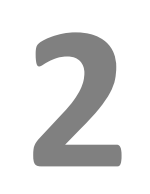

Development of a home visitation programme for the early detection of health problems in potentially frail community-dwelling older people by general practices

\section{Published as:}

Stijnen MMN, Jansen MWJ, Vrijhoef HJM, Duimel-Peeters IGP. Development of a home visitation programme for the early detection of health problems in potentially frail community-dwelling older people by general practices. Eur J Ageing, 2013;10:49-60. 


\section{ABSTRACT}

The integration within existing healthcare systems of preventive initiatives to maintain independent living among older people is increasingly emphasised. This article describes the development and refinement of the [G]OLD home visitation programme: an eight-step programme, including a comprehensive geriatric assessment, for the early detection of health and well-being problems among older people ( $\geq 75$ years) by general practices. A single group post-test study using a mixed model design is performed to evaluate (a) the feasibility of the home visitation programme in general practice, (b) the practical usefulness of the geriatric assessment instrument, and (c) programme implementation with respect to reinventions introduced by general practitioners (GPs) and practice nurses (PNs). Within three months' time, 22 PNs of 18 participating general practices visited 240 community-dwelling older people (mean age=82.0 years; $S D=4.2$ ) who had not been in contact with their general practice for more than six months. Mean time investment of the programme per older person was 118.1 minutes $(S D=27.0)$ for GPs and PNs combined. Evaluation meetings revealed that GPs and PNs considered the home visitation programme to be feasible in daily practice. They judged the geriatric assessment to be useful, although minor adjustments are needed (e.g., lay-out, substitution of tests). PNs often failed to register follow-up actions for detected problems in a care and treatment plan. Future training for PNs should address this issue. No reinventions were introduced that threatened fidelity of implementation. The findings are used to improve the home visitation programme before its evaluation in a large-scale controlled trial. 


\section{INTRODUCTION}

As the number of older people rises markedly worldwide, healthcare systems are faced with a growing number of potentially frail older people who suffer from complex, multiple health complaints. ${ }^{1,2}$ The changes in the demand of care among the ageing population accompanied by the increased healthcare costs prompted the focus on the timely identification of health risks among older people that may ultimately be detrimental for maintaining independent living. In Denmark, municipalities are enforced by state law to offer preventive in-home visits to older people aged 75 and over and several studies have been conducted to understand the best ways to organise the home visits and to determine its most important components. ${ }^{3}$ For example, cooperation between home care and general practitioners (GPS) is stimulated to organise efficient primary care for older people. ${ }^{4}$ In the Netherlands, preventive initiatives for older people are often insufficiently embedded within existing healthcare systems. ${ }^{2}$ More proactive, coherent and multidisciplinary preventive care using a patient-centred approach is needed. GPs, supported by a practice nurse (PN), are increasingly held responsible for this task by the Dutch Association for General Practitioners, ${ }^{5}$ as they sustain a generalist view and are easily accessible for older people. Hence, many GPs express the need of a structured approach for organising care for older people that fits into daily clinical practice.

\section{[G]OLD preventive home visitation programme}

This state of affairs has resulted in the development of a preventive home visitation programme for older people called [G]OLD: 'Getting OLD the healthy way', in two regions in the south of the Netherlands. While prior studies on the effectiveness of preventive home visits showed conflicting results, ${ }^{6,7}$ involving the GP seems a promising approach. ${ }^{4}$ We believe home visits can be valuable when embedded within general practices. It fosters multidisciplinary collaboration (between GPs, PNs and other caregivers), which is one of the key factors for successful care management of people with complex care needs. ${ }^{8}$ Moreover, strong primary care is associated with improved population health. ${ }^{9}$

We redesigned care delivery within general practices by applying components of the Chronic Care Model $(\mathrm{CCM})^{10}$ and the Guided Care Model, ${ }^{11}$ resulting in a comprehensive protocol that guides general practices in planning and executing geriatric care. Several of the included components have been postulated in reviews as promising components of interventions to prevent functional decline in community-dwelling older people. ${ }^{12,13}$ The [G]OLD-protocol applies a stepwise approach. First, the PN informs older people about the home visit using an information leaflet and makes an 
appointment for the home visit by phone (step 1). The PN prepares for the home visit by printing out relevant information from the GP's information system (e.g., medication list, medical history) (step 2). Then, the PN visits older people at home for a comprehensive geriatric assessment of their health and well-being using the so-called [G]OLD-instrument (step 3; see details below). Results of the assessment are discussed with the GP (step 4), as well as with the older person (step 5). In case problems or risk situations require attention, the PN and/or the GP formulate a care and treatment plan in accordance with the older person's needs and wishes (step 6). If applicable, the PN executes the care and treatment plan, for example by referring the older person to individually appropriate professionals (e.g., physiotherapist, occupational therapist) (step 7). In case different professionals need to be involved in follow-up actions, planning optimal care for the older person is discussed in regular multidisciplinary meetings. Finally, the PN monitors progress, and coordinates care and follow-up (step 8). Frequency of follow-up contacts will depend on the type of problems or complaints. When no follow-up actions are needed, the PN will check the older person's health status annually (by phone or by means of a re-assessment with the [G]OLDinstrument).

\section{[G]OLD geriatric assessment instrument}

Although many comprehensive geriatric assessment (CGA) instruments exist, general practices required a flexible instrument encompassing all components of interest to a GP to obtain a complete overview of the older person's health and well-being, while keeping the time investment to a minimum. Therefore, an expert panel (consisting of, among other, GPs) was brought together to develop a multidimensional geriatric assessment instrument: the [G]OLD-instrument (see Figure 2.1). It assists the PN in uncovering early signs of decline among relatively healthy older people and in identifying needs among older people with existing problems where decline is unavoidable; both with the ultimate goal to maintain independent living among older people.

The content of the [G]OLD-instrument is based on an existing Dutch instrument for geriatric assessment 'TRAZAG' ${ }^{14,15}$ and on input from the expert panel. The assessment covers 23 areas, including older people's functioning, physical and mental health, engagement in activities, and social well-being (for details, see Table 2.1). PNs can carry out an additional examination, or parts of it, if they require more insight into the presence or absence of a problem or risk situation. 


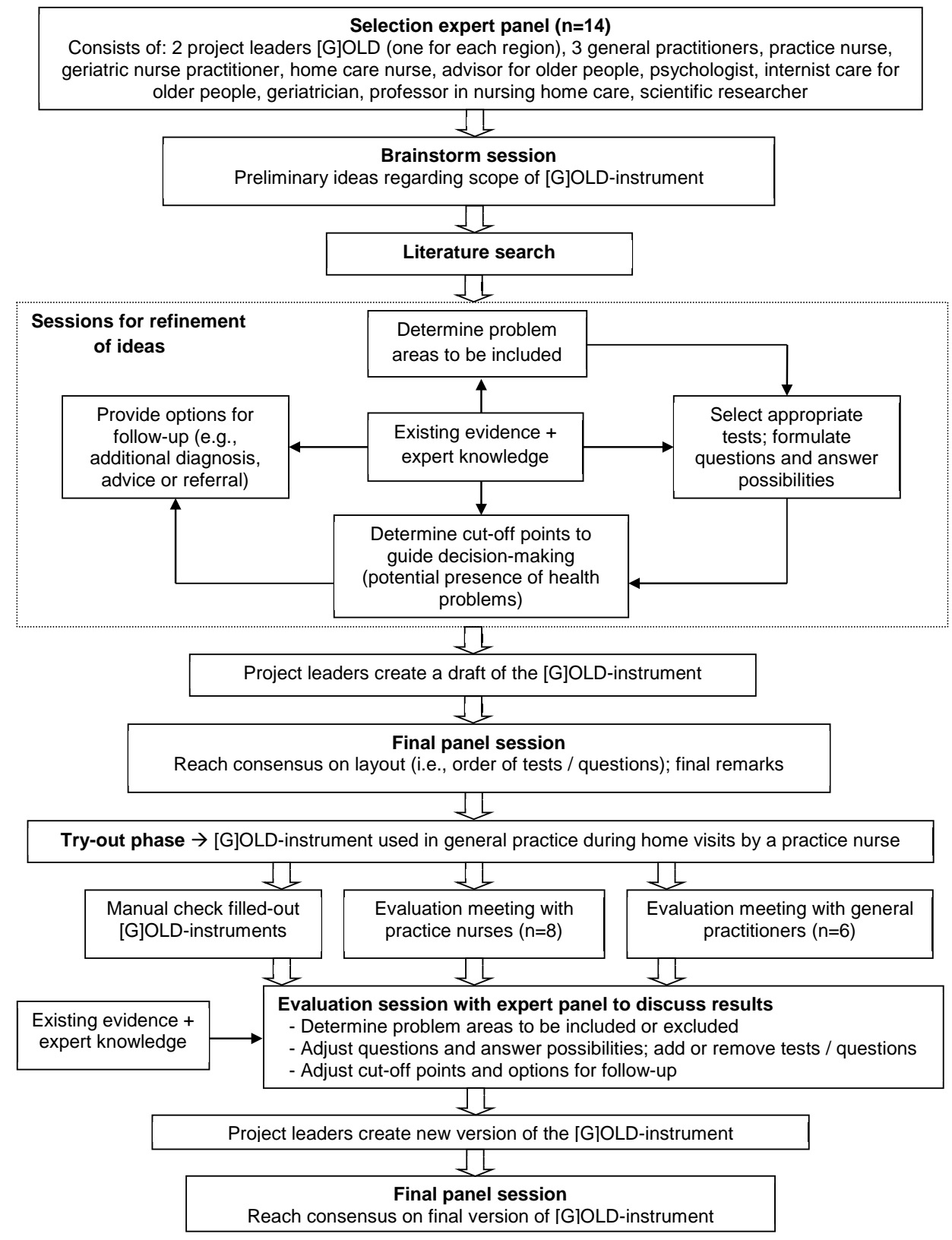

Figure 2.1 Flowchart procedure development multidimensional [G]OLD-instrument 
Table 2.1 Topics covered by the [G]OLD-instrument, their operationalisation and source

\begin{tabular}{|c|c|c|c|}
\hline Topics $^{a}$ & Operationalisation & (Preliminary) cut-off point & Source ${ }^{b}$ \\
\hline & PART ONE (basic assessment) & & \\
\hline \multirow{2}{*}{$\begin{array}{l}\text { General health } \\
\text { status }\end{array}$} & 1 item SF-20 ${ }^{16} ; 1$ item SF-36 ${ }^{17}$ & N/A & $\mathrm{EB}$ \\
\hline & 3 questions about complaints in past two months & N/A & PB \\
\hline Blood pressure & Blood pressure in $\mathrm{mmHg}$ & $\begin{array}{l}\mathrm{BP}_{\text {syst }}>140 \mathrm{mmHg} \\
\mathrm{BP}_{\text {diast }}>80 \mathrm{mmHg}\end{array}$ & biomed \\
\hline Medication use & $\begin{array}{l}\text { Number of prescribed drugs; comparison actual } \\
\text { use with prescription }\end{array}$ & $\geq 5$ prescribed drugs & PB \\
\hline IADL & IADL scale of Lawton and Brody ${ }^{18}$ & Dependent $\geq 1$ IADL & $\mathrm{EB} / \mathrm{PB}$ \\
\hline ADL & Barthel index ${ }^{19}$ & Dependent $\geq 1 \mathrm{ADL}$ & $\mathrm{EB} / \mathrm{PB}$ \\
\hline $\begin{array}{l}\text { Receiving } \\
\text { informal care }\end{array}$ & 2 questions about informal care giver & N/A & PB \\
\hline \multirow[t]{2}{*}{ Vision } & $\begin{array}{l}5 \text { questions (all self-report): } \\
\text { - } \quad \text { Problems in daily living because of impaired } \\
\text { sight; decrease in vision capability lately; } \\
\text { whether person wears glasses }\end{array}$ & Yes/No & PB \\
\hline & $\begin{array}{l}\text { Difficulty to read newspaper; difficulty to } \\
\text { recognise face at } 4 \text { meters distance }{ }^{20}\end{array}$ & Yes/No & EB \\
\hline Hearing & $\begin{array}{l}\text { Problems in daily living because of impaired } \\
\text { hearing; environment acknowledges impaired } \\
\text { hearing; whether person uses hearing aid (all self- } \\
\text { report) }\end{array}$ & Yes/No & PB \\
\hline \multirow[t]{2}{*}{ Nutrition } & Body Mass Index (BMI) - overweight ${ }^{21}$ & $\mathrm{BMI} \geq 25$ & biomed \\
\hline & $\begin{array}{l}\text { Undesired weight loss in past 3-6 months } \\
\text { Problems with eating and drinking }\end{array}$ & $\begin{array}{l}\text { Scored 'yes' on } 1 \text { item or } \\
\text { both items }\end{array}$ & PB \\
\hline Physical activity & $\begin{array}{l}\text { Physical activity on average } 30 \text { minutes per day, at } \\
\text { least } 5 \text { days per week }\end{array}$ & Yes/No & PB \\
\hline $\begin{array}{l}\text { Smoking } \\
\text { behaviour }\end{array}$ & $\begin{array}{l}\text { Whether person smokes and desire to quit } \\
\text { smoking }\end{array}$ & Yes/No & PB \\
\hline \multirow[t]{2}{*}{ Alcohol use } & $\begin{array}{l}\text { Whether person drinks alcohol; desire to } \\
\text { reduce/quit alcohol use }\end{array}$ & Yes/No & PB \\
\hline & 2 items to determine excessive alcohol use & $\begin{array}{l}<2 \text { days abstinent and } \\
\text { men } \geq 2-3 \text { glasses/day, } \\
\text { women } \geq 1-2 \text { gl./day }\end{array}$ & PB \\
\hline Memory & $\begin{array}{l}\text { Questions 1a-1e from Mini Mental State } \\
\text { Examination (MMSE) }\end{array}$ & $\geq 1$ wrong answer & EB \\
\hline Anxiety & $\begin{array}{l}\text { Part A of Hospital Anxiety and Depression Scale } \\
(\text { HADS })^{23}\end{array}$ & Score $\geq 8$ (range: $0-21$ ) & EB \\
\hline Depression & $\begin{array}{l}4 \text { items Geriatric Depression Scale }(G D S)^{24} \text { : } \\
\text { dissatisfaction; emptiness; depressed mood; } \\
\text { personal devaluation }\end{array}$ & Score $\geq 1$ (range: $0-4$ ) & EB \\
\hline
\end{tabular}




\begin{tabular}{|c|c|c|c|}
\hline Topics $^{a}$ & Operationalisation & (Preliminary) cut-off point & Source $^{b}$ \\
\hline $\begin{array}{l}\text { Personality } \\
\text { disorders }\end{array}$ & $\begin{array}{l}3 \text { items from Gerontologic Personality Disorders } \\
\text { Scale (GPS) }{ }^{25} \text { : intention to end life; suffering from } \\
\text { nervousness, tension; used sleeping pills in the past }\end{array}$ & $\begin{array}{l}\text { Score } \geq 2 \text { (range: } 0-3 \text { ) } \\
t\end{array}$ & $\mathrm{~PB}^{\mathrm{C}}$ \\
\hline Fall incidents & $\begin{array}{l}\text { Number of falls past } 6 \text { months } \\
\text { Cause of falls, concerned about falling, and } \\
\text { avoidance of activities due to fear of falling }\end{array}$ & $\begin{array}{l}\geq 1 \text { falls past } 6 \text { months } \\
\text { N/A }\end{array}$ & $\begin{array}{l}\text { PB } \\
\text { PB }\end{array}$ \\
\hline Mobility & Get-up and go test ${ }^{26}$ & Score $\geq 3$ (range: $0-5$ ) & $\mathrm{EB}$ \\
\hline $\begin{array}{l}\text { Participation in } \\
\text { society }\end{array}$ & Satisfaction with daily activities/routines & Yes/No & PB \\
\hline Loneliness & $\begin{array}{l}\text { Whether person feels lonely (grade } 1-10 \text { ) } \\
2 \text { items about cause of loneliness; need of support } \\
\text { to decrease loneliness }\end{array}$ & $\begin{array}{l}\text { Score } \geq 5 \text { (range: } 1-10 \text { ) } \\
\text { N/A }\end{array}$ & $\begin{array}{l}\text { PB } \\
\text { PB }\end{array}$ \\
\hline $\begin{array}{l}\text { Informal care } \\
\text { giving }\end{array}$ & $\begin{array}{l}\text { Provides informal care (and to whom) } \\
\text { Burdened by informal care giving }\end{array}$ & $\begin{array}{l}\text { Yes/No } \\
\text { Somewhat or very } \\
\text { burdened }\end{array}$ & $\begin{array}{l}\text { PB } \\
\text { PB }\end{array}$ \\
\hline Finance & Financial problems past 12 months & Yes/No & PB \\
\hline Quality of life & $\begin{array}{l}\text { Self-reported quality of life (grade 1-10) } \\
\text { PART TWO (additional examination) }\end{array}$ & N/A & PB \\
\hline Malnutrition & Mini Nutritional Assessment (MNA) ${ }^{27}$ & Score $\leq 11$ (range: 0-14) & EB \\
\hline Memory & $\begin{array}{l}\text { Complete Mini Mental State Examination (MMSE) } \\
\text { Clock drawing test }^{28}\end{array}$ & $\begin{array}{l}\left.{ }^{2} \text { Score } \leq 24 \text { (range: } 0-30\right) \\
\text { Score } \leq 4 \text { (range: } 0-5)\end{array}$ & EB \\
\hline Depression & Geriatric Depression Scale-15 (GDS-15) ${ }^{29}$ & Score $\geq 5$ (range: $0-15$ ) & $\mathrm{EB}$ \\
\hline $\begin{array}{l}\text { Personality } \\
\text { disorders }\end{array}$ & $\begin{array}{l}\text { Gerontologic Personality Disorders Scale (GPS) } \\
\text { consisting of HAB-scale and BIO-scale }\end{array}$ & $\begin{array}{l}\text { Score } \geq 5 \text { on } \mathrm{HAB}-\mathrm{scale} \\
\text { (range: } 0-7 \text { ) or BIO-scale } \\
\text { (range: } 0-9 \text { ) }\end{array}$ & $E B^{c}$ \\
\hline $\begin{array}{l}\text { Participation in } \\
\text { society }\end{array}$ & $\begin{array}{l}\text { Whether person regularly performs outdoor } \\
\text { activities (description activities and whether in } \\
\text { need of support) }\end{array}$ & Yes/No & PB \\
\hline \multirow[t]{2}{*}{$\begin{array}{l}\text { Informal care } \\
\text { giving }\end{array}$} & $\begin{array}{l}\text { Amount and type of informal care giving and } \\
\text { whether in need of support }\end{array}$ & N/A & PB \\
\hline & $\begin{array}{l}\text { Self-perceived Pressure from Informal Care test } \\
\text { (Dutch acronym: EDIZ) }^{30}\end{array}$ & $\begin{array}{l}\text { Scored 'yes' on item } 8 \text { and } \\
\text { 9: heavily burdened }\end{array}$ & $\mathrm{EB}$ \\
\hline
\end{tabular}

Note: N/A means not applicable. (I)ADL means (instrumental) activities of daily living.

${ }^{a}$ In the same order as they appear in the [G]OLD-instrument.

${ }^{\mathrm{b}} \mathrm{EB}=$ evidence-based; (derived from) an existing test validated for application in older subjects; $\mathrm{PB}=$ practice-based; formulated by expert panel based on clinical expertise or derived from clinical guidelines; $\mathrm{EB} / \mathrm{PB}=$ evidence-based/practice-based; an existing validated test, but slightly adjusted wording or answer possibilities to fit the current context; biomed = biomedical measurement.

${ }^{\mathrm{C}} \mathrm{A}$ validation study is being conducted to assess the validity and reliability of the complete version of the Gerontologic Personality Disorders Scale (GPS). 


\section{Objectives}

The [G]OLD home visitation programme was developed with the ultimate goal to provide general practices with a protocol for organising geriatric care that would be feasible and useful for application in daily practice. After the initial development, a pilot study was initiated to refine the [G]OLD home visitation programme for future use. The aims were to evaluate (a) the feasibility for general practices of home visits by a PN for the early detection of health and well-being problems, (b) the practical usefulness of the [G]OLD geriatric assessment instrument, and (c) whether the different steps of the [G]OLD home visitation programme were implemented as planned, by focusing on reinventions introduced by general practices. Reinvention may occur in the diffusion process when GPs and PNs apply the home visitation programme in their own practice and make changes to the original protocol (for further details, see Rogers ${ }^{31}$ ). Reinvention is often necessary for diffusing an innovation into a complex setting, although essential components of the innovation ensuring its effectiveness should not be discarded. It offers insight into how the [G]OLD home visitation programme can be put into daily practice on a large scale.

\section{METHODS}

\section{Study design and setting}

A cross-sectional study was performed (April-June 2009), with a single group post-test design in the Dutch regions Maastricht-Heuvelland and Parkstad. According to the Medical Research Involving Human Subjects Act, no formal ethical approval was needed for this study, as subjects were not required to follow rules of behaviour.

\section{Selection of general practices}

All general practices within the two regions $(n=143)$ were approached by postal mail to inform them about the study. They all had a PN working in the practice, but a prerequisite for participation was that the PN could devote time to conducting the home visits and arranging follow-up actions. A total of 21 general practices (14.7\%) agreed to participate ( 9 from Maastricht-Heuvelland, 12 from Parkstad) and were all included. 


\section{Selection of older people}

General practices selected community-dwelling people aged 75 years or older from the GP's Information System. Those on a waiting list for admission to a nursing home or a home for older people, and the terminally ill were excluded. All general practices were requested to purposefully select older people who had not been in contact with their GP/PN for a consultation or visit for more than six months before the start of this study. These 'apparently healthy' older people are often overlooked within general practice. However, they may fail to seek help or care for their unmet problems or complaints for various reasons. ${ }^{32}$ Therefore, this purposive sample might be particularly interesting for GPs as the results of the geriatric assessment might add to their current knowledge of the older person's health and well-being. PNs were allowed to select older people and, if required, GPs could check the final list. Eligible older people were then randomly approached by the PN in a stepwise manner (until the end of the three-month study phase) by means of an information leaflet by postal mail and subsequently a telephone call to schedule the home visit. General practices were informed that the time period for conducting the home visits for this study would be three months, but to decrease the risk of selection bias they could continue using the [G]OLD-instrument afterwards.

\section{Training practice nurses}

To equip PNs with the necessary knowledge and skills, they received two days of training. During the first day (prior to the pilot phase), information was provided concerning issues to be aware of in the ageing process. Supervised by a nurse practitioner (NP) specialised in care for older adults, PNs practiced applying the [G]OLD-instrument among nursing home residents. The second day (during the pilot phase) consisted of discussing PNs' practical experiences thus far and offering in-depth information on mental and psychological problems among older people, as well as impairments in hearing and sight. Additionally, a session focused on working in multidisciplinary teams and included meeting other professional caregivers or institutions offering care and/or well-being facilities for older people.

\section{Measures}

A mixed model design ${ }^{33}$ is applied. Equal priority is given to both quantitative and qualitative data, they are gathered concurrently, and mixing takes place both at the stages of data collection and integration (after data analysis). 


\section{Registration forms PNs}

PNs registered the average time required for step 1 to step 4 of the [G]OLD-protocol on a structured registration form. For the remaining steps, the time investment strongly depended on the number and type of problems detected. Reasons for nonparticipation among older people were registered on the same form. Results of the geriatric assessment were registered by PNs on a structured assessment form. At the end of each home visit, the PN asked the older person open-ended questions about his/her opinion concerning: (1) the [G]OLD-questions; (2) the duration of the home visit; and (3) whether they preferred a home visit or a consultation in the GP's practice, and recorded this in writing on a separate form. These are preliminary indicators of the acceptability of the home visitation programme according to older people. The [G]OLD-instrument also included a separate care and treatment plan containing a list with all problem areas from the [G]OLD-instrument for which further details and follow-up actions could be written down. This form was checked manually to identify inconsistencies between the number of detected problems and the number of followup actions formulated by the PN.

\section{Evaluation meetings}

Within one month after the pilot phase, semi-structured evaluation meetings were organised with 6 GPs (15.0\%) and 8 PNs (36.4\%) separately to evaluate the implementation of the different steps of the home visitation programme, including the feasibility of the home visitation programme, and the practical usefulness of the [G]OLDinstrument. One member of the project team led the discussion using a topic list, while another member of the project group made notes of the discussion. The topic list consisted of all eight steps of the home visitation programme, as well as the training of PNs, and overall time investment. After both meetings, the project team members discussed the notes and reached consensus on the interpretation of the discussion.

\section{Reinvention}

According to Rebchook and colleagues, ${ }^{34}$ three types of reinventions can be identified with different implications regarding fidelity (implementation as intended by the intervention developers): (1) a new component may be added to the protocol (no threat to fidelity); (2) minor changes to a component (fidelity more or less maintained or even improved); (3) major changes to or deletion of a component (fidelity threatened). For step 1 to step 6 of the [G]OLD-protocol, GPs and PNs were invited during the evaluation meetings to explain how they implemented each step. Any reinventions were subsequently labelled. Because the evaluation meetings took place shortly after the pilot phase to guarantee detailed information about experiences, no reinventions 
could be identified for step 7 and step 8 of the [G]OLD-protocol. A longer follow-up was not feasible within the study period.

\section{Data analysis}

Data were analysed using the statistical package SPSS for windows, version 17.0 (SPSS, Chicago, IL). Descriptive statistics (means, medians and frequencies) were computed for quantitative variables. The number of planned follow-up actions following problems or complaints detected during the geriatric assessment were obtained by means of $2 \times 2$ contingency tables for each topic in the [G]OLD-instrument.

Content analysis was performed on the qualitative data from the registration forms, care and treatment plans, and the open-ended questions in the [G]OLDinstrument. The results of the evaluation meetings with GPs and PNs were summarised in a narrative report.

\section{RESULTS}

\section{Participants}

One general practice dropped out at the start of the study due to illness of the PN. Two general practices did not return the forms before deadline. Thus, we included data from 18 general practices. Five of these were solo practices, five practices with two GPs, and the remaining were group practices ( $\geq 3 \mathrm{GPs}$ ) (total $n=40 \mathrm{GPs}$ ).

In total, 22 PNs invited 272 eligible older people for a home visit, of which 32 (11.8\%) refused to participate. Reasons for refusal were: visit not necessary (not in need of (additional) help or care) (57.6\%), not interested in visit/medical examination (12.1\%), suspicion (e.g., afraid to be placed in a home for older people) (9.4\%), or other reasons (does not want results to be used for research; illness; partner passed away; could not be reached by phone; informal caregiver insulted; already had visit from a consultant for older people) (18.2\%). The average refusal rate per general practice was $12.5 \%$ (SD=13.7; range: $0 \%-50 \%$ ).

PNs visited 240 participants at home within a three-month period for the [G]OLD geriatric assessment. The average number of home visits conducted per PN was 10.9 visits ( $S D=5.1$; range: $2-20$ ). An overview of the participants' socio-demographic characteristics is shown in Table 2.2. The mean age was 82.0 years; the majority was female, widowed, lived alone, and had completed primary education. 
Table 2.2 Socio-demographic characteristics of older people enrolled in study $(n=240)$

\begin{tabular}{lll}
\hline & Years & $\mathrm{n}(\%)$ \\
\hline Age & & \\
Mean \pm SD & $82.0 \pm 4.2$ & \\
Range & $75-94$ & \\
Median & 82.0 & \\
Gender & & $164(68.9 \%)$ \\
Female & \\
Marital status & \\
Single, widowed & $149(62.1 \%)$ \\
Married & $68(28.3 \%)$ \\
Single, divorced & $11(4.6 \%)$ \\
Single, never married & $9(3.8 \%)$ \\
Living together, unmarried & $3(1.2 \%)$ \\
Living status & \\
Living alone & $158(65.8 \%)$ \\
Living with partner, husband/wife & $68(28.3 \%)$ \\
Living with others & $14(5.9 \%)$ \\
Level of education & \\
Primary education & $116(49.1 \%)$ \\
Lower to middle professional education & $108(45.8 \%)$ \\
Higher professional education & \\
\hline Note: SD Standard Devition: & \\
\hline
\end{tabular}

Note: $\mathrm{SD}=$ Standard Deviation; ${ }^{\mathrm{a}} \mathrm{n}=236$

\section{Feasibility [G]OLD home visitation programme}

Time investment

The average total time investment for GPs and PNs combined was 118.1 minutes (for details, see Table 2.3). The home visit itself took on average 55.7 minutes and the duration of the home visit varied considerably between PNs. 
Table 2.3 Time required for the different elements of the [G]OLD preventive home visitation programme according to practice nurses $(n=19)$

Elements [G]OLD preventive home visitation programme

Average time investment GP and PN per older person (minutes)

\begin{tabular}{lccc} 
& M & SD & Range \\
\cline { 2 - 4 } Total time selection of eligible older people & 9.3 & 8.1 & $1.0-30.0$ \\
Selection of eligible older people by GP & 2.2 & 2.4 & $0.0-8.0$ \\
Selection of eligible older people by PN & 7.1 & 8.5 & $0.0-30.0$ \\
Send older people the invitation letter and information leaflet (PN) & 8.6 & 3.6 & $2.0-15.0$ \\
Make an appointment with the older person by phone for the home visit (PN) & 7.7 & 4.5 & $2.0-15.0$ \\
Selection of relevant information from the GP's Information System as & 6.1 & 3.9 & $0.5-15.0$ \\
preparation for the home visit (PN) & & & \\
Travel back and forth for the home visit (PN) & 15.8 & 6.0 & $5.0-30.0$ \\
Duration of the home visit (PN) & 55.7 & 14.5 & $34.0-92.5$ \\
Post-discussion GP and PN & 8.1 & 5.39 & $0.8-21.5$ \\
Total average time investment (GP + PN) & $\mathbf{1 1 8 . 1}$ & $\mathbf{2 7 . 0}$ & $\mathbf{7 8 . 4 - \mathbf { 1 6 }}$
\end{tabular}

Note: $\mathrm{M}=$ Mean; $\mathrm{SD}=\mathrm{Standard}$ Deviation

Feasibility according to GPs and PNs

In general, GPs indicated that due to [G]OLD they experienced an improvement in quality of care for older people and an intensification of the contacts between PNs and older people.

GPs all agreed that the home visits are time consuming and that the costs are not fully reimbursed by health insurers. Some PNs struggled with planning the home visits, because it was difficult to judge on forehand how long a home visit would take. Nevertheless, all GPs and PNs preferred a home visit instead of a consultation in the GP's practice. It provides useful information about living conditions and the functioning of older people in their daily environment. GPs argued that it is important to investigate for which community-dwelling older people (aged $\geq 75$ years) the geriatric assessment is most beneficial.

With respect to the group of non-responders, both GPs and PNs agreed that a home visit would have been particularly beneficial for these people. They might avoid seeking care or maintain a façade of "normalcy" to prevent others from noticing their problems. According to PNs, this group of older people is difficult to reach and they suggest that GPs should try to contact refusers.

Finally, PNs wished more guidelines regarding follow-up actions that can be undertaken following detected problems or needs. GPs indicated that a service map with an overview of (local) health and well-being facilities would be useful and desirable. 


\section{Multidimensional geriatric assessment}

Follow-up actions for detected health or well-being needs

According to the cut-off points in the basic assessment, follow-up actions were needed among 238 older people (99.2\%). Although required, for $10.9 \%$ of these older people $(n=26)$ no care and treatment plan was formulated by the PN. Reasons for this are mainly unknown, although in general these older people seem to experience somewhat fewer problems (e.g., fewer complaints in the past two months, less dependent in IADL) compared to people for whom care and treatment plans were formulated. The number of follow-up actions formulated in the care and treatment plan per detected problem or risk situation is shown in Table 2.4. Most follow-up actions were following positive test results with respect to memory problems (58.7\%) (e.g., performing the MMSE test), decreased mobility (53.8\%) (e.g., referral to physiotherapist or occupational therapist), and depression (50.7\%) (e.g., performing the GDS-15 test), whereas for financial problems, excessive alcohol use, and dependency in one or more instrumental activities of daily living, no or only few follow-up actions were registered $(0 \%$, $0 \%$, and $7.4 \%$, respectively). For only 69 out of all 824 unaddressed problems or needs, PNs wrote down the reason for not undertaking follow-up actions. Mostly, older people already received treatment, care or help (43.5\%) for problems such as impaired hearing, impaired sight, and dependency in (instrumental) activities of daily living. Furthermore, some older people wished no action (33.3\%), such as in case of impaired hearing and smoking. In other cases no improvement was possible (13.0\%; problems such as polypharmacy or impaired sight) or the GP applied a "watchful waiting" policy (10.1\%; problems such as depression or one fall incident in past six months).

PNs used part two of the [G]OLD-instrument for $32.1 \%$ of the older people $(n=77)$ and it took on average 28.7 minutes to complete $(S D=14.9$; range: $5.0-70.0$, large variation due to differences in tests that were executed). Follow-up actions formulated per detected problem or need are presented in Table 2.4. Again, for some problems, no follow-up actions were formulated and the only reason mentioned in 8 out of 25 unaddressed problems was that the older person refused action. Note the discrepancy in the number of older people who underwent certain tests during the additional examination and the number of older people who should have undergone these tests according to the prevalence numbers in the basic assessment. This implies that PNs did not always perform the additional examination or parts of it. 
Table 2.4 Follow-up actions formulated in the care and treatment plan following positive test results in the basic assessment and additional examination using the [G]OLD-instrument

\begin{tabular}{|c|c|c|}
\hline (Early signs of) problems ${ }^{a}$ & $\begin{array}{l}\text { Prevalence, } \\
\mathrm{n}(\%)\end{array}$ & $\begin{array}{l}\text { Follow-up actions, } \\
\mathrm{n}(\%)^{\mathrm{b}}\end{array}$ \\
\hline
\end{tabular}

Basic assessment ([G]OLD-instrument part one) ( $\mathrm{n}=\mathbf{2 4 0})$

Hypertension $(n=230)$

$160(69.6 \%) \quad 54(33.8 \%)$

Polypharmacy $(n=155)^{c}$

$67(43.2 \%)$

$12(17.9 \%)$

Dependent $\geq 1$ IADL ( $n=240)$

$94(39.2 \%)$

$7(7.4 \%)$

Insufficient physical activity $(\mathrm{n}=239)$

$84(35.1 \%)$

$12(14.3 \%)$

Decreased mobility $(n=76)^{c}$

$26(34.2 \%)$

$14(53.8 \%)$

Problems daily living due to impaired hearing $(n=239)$

$79(33.1 \%)$

$36(45.6 \%)$

Problems daily living due to impaired sight $(n=238)$

74 (31.1\%)

$26(35.1 \%)$

Depression $(n=235)$

$69(29.4 \%)$

$35(50.7 \%)$

Moderate to severe overweight $(n=223)$

$61(27.4 \%)$

7 (11.5\%)

Falls in last 6 months $(n=239)$

$65(27.2 \%)$

$19(29.2 \%)$

Dependent $\geq 1$ ADL $(n=240)$

$65(27.1 \%)$

$5(7.7 \%)$

Loneliness $(n=237)$

$61(25.7 \%)$

$10(16.4 \%)$

Cognitive impairment ( $n=223$ )

$46(20.6 \%)$

$27(58.7 \%)$

Excessive alcohol use $(n=100)^{c}$

$20(20.0 \%)$

$0(0 \%)$

Burdened by informal care giving $(n=36)^{c}$

7 (19.4\%)

$1(14.3 \%)$

Unsatisfied with daily routines $(n=235)$

$41(17.4 \%)$

$12(29.3 \%)$

Smoking $(n=237)$

$33(13.9 \%)$

$11(33.3 \%)$

Personality disorders $(n=237)$

$29(12.2 \%)$

$14(48.3 \%)$

Problems living conditions or housing $(n=239)$

$28(11.7 \%)$

$7(25.0 \%)$

Anxiety $(n=225)$

$17(7.6 \%)$

$7(41.2 \%)$

Financial problems $(n=208)$

$9(4.3 \%)$

$0(0 \%)$

Malnutrition $(n=238)$

$6(2.5 \%)$

$1(16.7 \%)$

Additional examination ([G]OLD-instrument part two) $(n=77)$

Malnutrition $(n=4)$

$\begin{array}{ll}3(75.0 \%) & 2(66.7 \%) \\ 25(69.4 \%) & 15(60.0 \%) \\ 21(51.2 \%) & 13(61.9 \%) \\ 5(34.2 \%) & 2(40.0 \%) \\ 1(33.3 \%) & 0(0 \%) \\ 3(17.6 \%) & 1(33.3 \%)\end{array}$

Cognitive impairment $(n=36)$

Depression $(n=41)$

Unsatisfied with daily routines $(n=17)$

Burdened by informal care giving $(n=3)$

Personality disorders $(n=17)$

$3(17.6 \%)$

$1(33.3 \%)$

Note: (I)ADL means (instrumental) activities of daily living.

${ }^{\text {a }} \mathrm{N}$ representing number of participants who underwent that specific test or set of questions.

${ }^{\mathrm{b}} \mathrm{N}$ and \% of total number of participants with the (potential) problem (column 'Prevalence').

${ }^{\mathrm{c}} \mathrm{N}$ representing number of participants from total sample who use medications, have difficulty walking around the house during the home visit, drink alcohol, and provide informal care, respectively. 


\section{Practical usefulness according to GPs and PNs}

In general, PNs and GPs judged the [G]OLD-instrument to be practically useful. One GP presented a case in which the [G]OLD-instrument helped to get a complete overview of an older person's health status which would facilitate a quicker transition to a nursing facility when the situation becomes unbearable. The GPs agreed that older people are a heterogeneous group and that a broad, structured geriatric assessment instrument is necessary. PNs discussed the more practical side of administrating the [G]OLDinstrument. All suggested improvements by GPs and PNs were grouped into the following categories (random order): (a) lay-out; (b) textual errors; (c) removal of tests or questions (e.g., Whispered Voice test); (d) adding new tests or questions (e.g., type of house); (e) substitution of tests or questions by other, more valid or more reliable, alternatives (e.g., ADL and IADL questions by GARS ${ }^{35}$ ); ( $f$ ) changes in the order of questions. Since no changes were made during the pilot phase, we do not label these suggestions as 'reinventions'.

PNs indicated that they required additional instructions regarding the administration of tests in the [G]OLD-instrument and clearer cut-off points for (early signs of) problems. For example, they emphasised the need for additional training in assessing older people's medication use. GPs also acknowledged the importance of good training and guidelines for PNs. For instance, the MMSE test for determining cognitive impairment can be very confronting for older people if not introduced well by the PN.

\section{Reinvention}

Both GPs and PNs present at the evaluation meetings considered all steps of the structured [G]OLD-protocol to be valuable. For example, GPs acknowledged the importance of formulating a care and treatment plan with clear follow-up actions since this is essential for ensuring an adequate follow-up.

One PN made a minor change in step 1, namely mentioning the date of the home visit already in the first letter sent to the older person instead of making the appointment after sending the letter. One PN indicated not having sent the letter and the information leaflet to the older people (not implemented). This step was purposefully omitted from the protocol by the PN and substituted by directly calling the older person for making an appointment. Although the other PNs did send the letter with the information leaflet, they questioned whether older people actually read the leaflet. In addition to the protocol, all PNs also registered the results of the geriatric assessment in the GP's information system. They consider this essential for the follow-up of older people over time. Finally, two GPs mentioned having pre-discussed each planned visit with the PN (addition to protocol), while the other GPs explicitly mentioned not having 
pre-discussed the visits, because the PN should keep an open mind during the assessment.

Besides these reinventions, all other steps of the [G]OLD home visitation programme were implemented as planned according to PNs and GPs.

\section{Acceptability of home visits according to older people}

Of the participants, $95.3 \%$ were satisfied about the duration of the visit, $0.9 \%$ thought it was too short, and $3.8 \%$ gave no opinion. The majority preferred a home visit $(83.8 \%)$ instead of a consultation in the GP's practice (1.3\%), $14.9 \%$ felt indifferent. In general, participants judged the [G]OLD-questions asked to be "fine" $(85.8 \%)$, meaning that they were acceptable, while $4.3 \%$ had no opinion and $5.7 \%$ gave other comments. Only $3.4 \%$ found them hard to answer, $0.4 \%$ thought they were unpleasant, and $0.4 \%$ said they were too personal.

\section{DISCUSSION}

The present study demonstrates that testing an intervention before its large-scale implementation provides valuable information for understanding how the intervention functions in the intended context. The Medical Research Council has argued that this often-neglected phase is essential in developing and evaluating complex interventions aimed at improving health, as a failure to consider the practical issues of implementation properly will lead to weaker interventions. ${ }^{36}$

Compared to other recent studies in the Netherlands in which the effect of the preventive home visits was absent, ${ }^{37,38}$ the [G]OLD home visitation programme is fully embedded within general practices. It comprises elements of the Guided Care model that has shown to be feasible and acceptable for patients and caregivers. ${ }^{39}$ In contrast to interventions with a resource-based approach that aim to achieve and maintain overall well-being by stimulating self-management abilities, ${ }^{40}$ the current problemoriented approach fits more closely to the available knowledge and expertise in general practices. We found that the [G]OLD home visitation programme is indeed feasible for GPs and PNs, and also acceptable for older people. Although GPs considered the home visitation programme to be time consuming (average time investment per patient was almost two hours), they emphasised the improved quality of care for their older patients. However, feasibility cannot be guaranteed over an extended period, as insufficient reimbursement by health insurers of the costs of this type of care for older people may eventually start to outweigh the benefits. 
The [G]OLD-instrument was judged to be useful by both GPs and PNs. They gave valuable comments (such as clearer cut-off points) which will lead to improvement of the [G]OLD-instrument (see final steps in Figure 2.1). Many health or well-being problems were detected for which follow-up actions were formulated in the care and treatment plan. This suggests, as concluded by previous research in other countries, ${ }^{41,42}$ that a geriatric assessment in general practice might be worthwhile in terms of identifying previously unknown problems. Furthermore, the fact that many (early signs of) problems were detected among older people who had not visited the GP/PN for more than six months suggests that older people may not seek help or underreport symptoms, problems or needs. Potential reasons for this are that older people attribute them as natural features of ageing, or symptoms that might indicate psychiatric disorders (e.g., low mood, forgetfulness) may not be considered to represent disease that requires a medical opinion. ${ }^{43}$ Due to the aim of our study, we did not intend to obtain a representative sample of all community-dwelling older people who rarely consult their GP and we did not collect data among older people who often consulted the GP. Therefore, the prevalence numbers should be interpreted with caution. Furthermore, it is deemed unlikely that general practices selected a more frail subpopulation of older people for the home visits, as they were informed prior to the study that they could continue using the [G]OLD-instrument afterwards. It might be argued that the cut-off points in the [G]OLD-instrument should be raised since for several detected needs follow-up actions were lacking. However, the expert panel judged the cut-off points to be appropriate. If there are early signs of problems, but follow-up actions are not desired or necessary yet according to the older person or GP, the GP can keep this information in mind for future reference.

Besides the suggested changes to the [G]OLD-instrument, this study also provided insight into necessary adjustments to improve the home visitation programme in general and issues to be aware of during its large-scale implementation. For example, responders might be healthier than non-responders, which indicates the importance of examining differences between the two groups in future research. Moreover, we noticed that PNs sometimes skipped certain tests or questions in the assessment for unknown reasons. In addition, PNs often did not clearly register problems and/or complaints detected during the home visit in the care and treatment plan or they did not (yet) specify appropriate follow-up actions. Possible explanations are that PNs, as indicated during the evaluation meetings, need clearer cut-off points and more guidelines for follow-up actions, they might register follow-up actions elsewhere (e.g., the GP's Information System), or no follow-up actions were needed. Even when follow-up actions were formulated, they often lacked detail (e.g., "monitor progress"). Without appropriate follow-up actions after the detection of health and well-being needs, the effectiveness of the home visitation programme may be compromised. It is likely that 
the two-day training has been too short and may not have addressed these and other aspects (e.g., how to build a good relationship with the older person) adequately. Future training for PNs should concentrate on these aspects and in particular the issue of inaccurate/inadequate reporting by PNs.

Our focus on reinventions additionally resulted in allowing PNs to mention the date of the home visit already in the invitation letter send to the older person. Further, despite critical comments on the information leaflet for older people, the leaflet is essential in terms of informed consent and therefore should not be omitted. The majority of GPs disagreed with a pre-discussion before the home visit and therefore we did not include this as an additional component to the [G]OLD-protocol. Finally, we aim to develop an ICT-based solution for general practices in which PNs can register the results of the geriatric assessments and plan follow-up actions in a uniform way.

Although this study refers to a specific intervention in a specific context, the results may contribute to an improved understanding of the issues to be aware of in the early phase of intervention implementation (e.g., reinventions introduced by programme implementers, inaccurate reporting). Furthermore, we illustrated the steps undertaken in designing and testing the intervention protocol as well as the geriatric assessment instrument. A limitation of the current study is that only few professionals attended the evaluation meetings and especially GPs were underrepresented. However, we did receive valuable information and critical comments which will help to improve both the home visitation programme and the [G]OLD-instrument, although it is likely that we missed certain opinions. Furthermore, this study provided insufficient insight into whether planned follow-up actions were indeed undertaken and to what extent other professionals were involved. A thorough process evaluation as part of our upcoming controlled trial will further address this specific issue.

In conclusion, the preliminary results of this study are promising and the [G]OLD preventive home visitation programme seems to be a valuable tool for general practices. Adjustments to the [G]OLD-instrument and the training for PNs are needed before further implementation. A large-scale controlled trial will be conducted to evaluate the influence of the home visitation programme on older people's health-related quality of life and disability, and to evaluate into more detail the implementation of all elements of the [G]OLD-protocol within general practices, including the satisfaction of care providers and older people (process evaluation). This type of effectiveness studies is needed to facilitate the translation of research into practice and to increase the likelihood that evidence-based interventions will be adopted within the healthcare system. $^{44}$ 


\section{REFERENCES}

1. Blokstra A, Baan CA, Boshuizen HC, Feenstra TL, Hoogenveen RT, Picavet HSJ, et al. Vergrijzing en toekomstige ziektelast. Prognose chronische ziektenprevalentie 2005-2025 [Impact of the ageing population on burden of disease. Projections of chronic disease prevalence for 2005-2025] Bilthoven: RIVM, 2007.

2. Health Council of the Netherlands. Health care for the elderly with multimorbidity. The Hague: Health Council of the Netherlands, 2008 Publication no. 2008/01.

3. Rubenstein LZ. New insights from the Danish preventive home visit trial. Eur J Ageing 2007;4:141-143.

4. Vass M, Avlund K, Siersma V, Hendriksen C. A feasible model for prevention of functional decline in older home-dwelling people-the GP role. A municipality-randomized intervention trial. Fam Pract 2009;26:56-64.

5. Dutch College of General Practitioners. NHG-Standpunt Toekomstvisie Huisartsenzorg. Huisartsgeneeskunde voor ouderen [Statement of the NHG, Future of General Practitioner Care. General practice medicine for older people]. Utrecht: NHG, 2007.

6. Van Haastregt JCM, Diederiks JPM, Van Rossum E, De Witte LP, Crebolder HFJM. Effects of preventive home visits to elderly people living in the community: systematic review. Br Med J 2000;320:754-758.

7. Elkan R, Kendrick D, Dewey M, Hewitt M, Robinson J, Blair M, et al. Effectiveness of home based support for older people: systematic review and meta-analysis. Br Med J 2001;323:719-725.

8. Bodenheimer T, Berry-Millett R. Care management of patients with complex health care needs. Princeton: Robert Wood Johnson Foundation 200919.

9. Starfield B, Shi L, Macinko J. Contribution of primary care to health systems and health. Milbank $Q$ 2005;83:457-502.

10. Bodenheimer T, Wagner EH, Grumbach K. Improving primary care for patients with chronic illness. J Am Med Assoc 2002;288:1775-1779.

11. Boult C, Karm L, Groves C. Improving chronic care: the "Guided Care" model. Perm J 2008;12:50-54.

12. Daniels R, Metzelthin S, Van Rossum E, De Witte L, Van den Heuvel W. Interventions to prevent disability in frail community-dwelling older persons: an overview. Eur J Ageing 2010;7:37-55.

13. Markle-Reid M, Browne G, Weir R, Gafni A, Roberts J, Henderson SR. The effectiveness and efficiency of home-based nursing health promotion for older people: a review of the literature. Med Care Res Rev 2006;63:531-569.

14. Warnier RMJ. TRAZAG. Transmuraal Zorg Assessment Geriatrie [TRAZAG. Transmural Care Assessment Geriatrics]. Maastricht: Academic Hospital Maastricht / MUMC+; 2008.

15. Warnier RMJ, Debie T, Beusmans G. Complexe zorg voor ouderen. Beoordelingsinstrument voor de praktijkverpleegkundige [Complex care for older people. Assessment instrument for the practice nurse]. Tijdschr Prakt 2007;2:50-53.

16. Kempen GIJM, Brilman El, Heyink JW, Ormel J. Het meten van de algemene gezondheidstoestand met de MOS Short-Form General Health Survey (SF-20): een handleiding [Measurement of general health status with the MOS Short-Form General Health Survey (SF-20): a guideline]. Groningen: Rijksuniversiteit Groningen, Noordelijk Centrum voor Gezondheidsvraagstukken, 1995.

17. Van der Zee KI, Sanderman R. Het meten van de algemene gezondheidstoestand met de RAND-36: een handleiding [Measurement of general health status with the RAND-36: a guideline]. Groningen: Rijksuniversiteit Groningen, Noordelijk Centrum voor Gezondheidsvraagstukken, 1993.

18. Lawton MP, Brody EM. Assessment of older people: self-maintaining and instrumental activities of daily living. Gerontologist 1969;9:179-186.

19. Mahoney FI, Barthel DW. Functional evaluation: the Barthel Index Md State Med J 1965;14:61-65.

20. Van Sonsbeek JLA. Methodological and substantial aspects of the OECD indicator of functional limitations. Maandbericht Gezondheid (CBS) 1988;88:4-17. 
21. World Health Organization. Obesity: preventing and managing the global epidemic. Geneva, Switzerland: World Health Organization, 2000 WHO Technical Report Series 894.

22. Folstein MF, Folstein SE, McHugh PR. "Mini-mental state". A practical method for grading the cognitive state of patients for the clinician. J Psychiatr Res 1975;12:189-198.

23. Zigmond AS, Snaith RP. The hospital anxiety and depression scale. Acta Psychiatr Scand 1983;67:361370.

24. Yesavage JA, Brink TL, Rose TL, Lum O, Huang V, Adey M, et al. Development and validation of a geriatric depression screening scale: a preliminary report. J Psychiatr Res 1982;17:1982-1983.

25. Van Alphen SP, Engelen GJ, Kuin Y, Hoijtink H, Derksen JJ. Constructie van een schaal voor de signalering van persoonlijkheidsstoornissen bij ouderen [Construction of a scale to detect personality disorders in older people]. Tijdschr Gerontol Geriatr 2004;35:186-195.

26. Mathias S, Navak US, Isaacs B. Balance in elderly patients: the "get-up and go" test. Arch Phys Med Rehabil 1986;67:387-389.

27. Guigoz Y, Yellas B, Garry PJ. Mini Nutritional Assessment: a practical assessment tool for grading the nutritional state of elderly patients. Facts Res Gerontol 1994;4 (suppl 2):15-60.

28. Shulman K, Shedletsky R, Silver IL. The challenge of time: clock-drawing and cognitive function in the elderly. Int J Geriatr Psychiatry 1986;1:135-140.

29. Sheikh JA, Yesavage JA. Geriatric Depression Scale (GDS): recent findings and development of a shorter version. In: Brink TL, editor. Clinical Gerontology: A Guide to Assessment and Intervention. New York: Howarth Press; 1986.

30. Pot AM, Van Dijck R, Deeg DJ. Ervaren druk door informele zorg: constructie van een schaal [Perceived stress caused by informal caregiving: construction of a scale]. Tijdschr Gerontol Geriatr 1995;26:214219.

31. Rogers EM. Diffusion of innovations. 5th ed. New York: Free Press; 2003.

32. Walters K, Iliffe S, Orrell M. An exploration of help-seeking behaviour in older people with unmet needs. Fam Pract 2001;18:277-282.

33. Johnson RB, Onwuegbuzie AJ. Mixed methods research: a research paradigm whose time has come. Educ Res 2004;33:14-26.

34. Rebchook GM, Kegeles SM, Huebner D, TRIP Research Team. Translating research into practice: the dissemination and initial implementation of an evidence-based HIV prevention program. AIDS Educ Prev 2006;18:119-136.

35. Kempen GIJM, Miedema I, Ormel J, Molenaar W. The assessment of disability with the Groningen Activity Restriction Scale. Conceptual framework and psychometric properties. Soc Sci Med 1996;33:1601-1610.

36. Craig P, Dieppe P, Macintyre S, Mitchie S, Nazareth I, Petticrew M. Developing and evaluating complex interventions: the new Medical Research Council guidance. Br Med J 2008;337:a1655.

37. Bouman A, Van Rossum E, Ambergen T, Kempen GIJM, Knipschild P. Effects of a preventive home visiting program for older people with poor health status: a randomized clinical trial in the Netherlands. J Am Geriatr Soc 2008;56:397-404.

38. Van Hout HPJ, Jansen APD, Van Marwijk HWJ, Nijpel G. Prevention of adverse health trajectories in a vulnerable elderly population through nurse home visits: randomized controlled trial [ISRCTN05358495]. J Gerontol A Biol Sci Med Sci 2010;65:734-742.

39. Boyd CM, Boult C, Shadmi E, Leff B, Brager R, Dunbar L, et al. Guided care for multimorbid older adults. Gerontologist 2007; 47:697-704

40. Steverink N, Lindenberg S, Slaets JPJ. How to understand and improve older people's self-management of wellbeing. Eur J Ageing 2005;2:235-244.

41. Lucchetti G, Granero AL. Use of comprehensive geriatric assessment in general practice: results from the 'Senta Pua' project in Brazil. Eur J Gen Pract 2011;17:20-27. 
42. Piccoliori G, Gerolimon E, Abholz H. Geriatric assessment in general practice using a screening instrument: is it worth the effort? Results of a South Tyrol Study. Age Ageing 2008;37:647-652.

43. Morgan R, Pendleton N, Clague JE, Horan MA. Older people's perceptions about symptoms. Br J Gen Pract 1997;47:427.

44. Gill TM. Education, prevention, and the translation of research into practice. J Am Geriatr Soc 2005;53:724-726. 


\section{Process evaluation plan of a patient-centred home visitation program for potentially frail community-dwelling older people in general practice}

Published as:

Stijnen MMN, Duimel-Peeters IGP, Vrijhoef HJM, Jansen MWJ. Process evaluation plan of a patient-centred home visitation program for potentially frail community-dwelling older people in general practice. Eur J Pers Cent Healthc, 2014;2:179-189. 


\section{ABSTRACT}

\section{Background}

Evaluation studies examining the effectiveness of interventions tend to offer little insight into the mechanisms responsible for changes in outcomes. Therefore, a thorough process evaluation is conducted alongside a longitudinal quasi-experimental trial investigating the effects of a home visitation programme for the early detection of health problems among potentially frail community-dwelling older people ( $\geq 75$ years). We aim to describe the rationale for and steps undertaken in developing a process evaluation plan to identify the factors that influence the success or failure of this complex, patient-centred intervention within the primary care setting.

\section{Method}

Using a theoretical framework underlying the process evaluation, process evaluation questions are formulated per component of the framework (i.e., implementation fidelity, dose delivered, dose received, reach, recruitment, and context). The process evaluation plan shows how both quantitative (e.g., structured registration forms) and qualitative methods (e.g., semi-structured interviews) are applied in gathering process data for a complex, patient-centred intervention integrated within general practices. Process data are gathered with either formative or summative purposes among practice nurses and general practitioners from participating general practices and a purposive sample of older people.

\section{Conclusion}

Conducting a process evaluation alongside a clinical trial will assist in deciding to what extent the intervention is effective, as well as what factors contribute to the intervention's effectiveness. The insights gained are imperative for the development of patientcentred interventions that are likely to be sustained when implemented in the intended context. 


\section{INTRODUCTION}

Most evaluation studies are primarily concerned with obtaining robust evidence of the effectiveness of interventions, while offering little insight into the underlying mechanisms through which changes in outcomes may occur. As a result, there has been a growing interest in process evaluation to understand the steps undertaken during implementation of an intervention. Process evaluation data help to identify factors responsible for the success or failure of an intervention, including for whom and under what circumstances the intervention is effective. ${ }^{1}$ Hence, process evaluation looks inside the "black box" to differentiate between interventions that have shortcomings in itself (intervention failure) or interventions that are badly delivered (implementation failure). ${ }^{2,3}$

Although process evaluation is frequently applied in the field of public health to evaluate health promotion and disease prevention programmes, ${ }^{1}$ its merit for the evaluation of patient-centred healthcare interventions within the primary care setting is increasingly recognised as well. Primary care studies incorporating a process evaluation predominantly focus on implementing evidence-based interventions or clinical guidelines into practice. For example, a process evaluation conducted by Flottorp and colleagues ${ }^{4}$ revealed reasons why general practices failed to implement guidelines for sore throat and urinary tract infections. In general, primary healthcare professionals experience various barriers to the implementation of evidence-based research findings into everyday practice. ${ }^{5,6}$ Process evaluation can assist in identifying these barriers at an early stage, which may allow interventions to be disseminated and adopted more successfully ${ }^{7}$ after their effectiveness has been established.

Nevertheless, the use of qualitative methods alongside controlled trials of complex healthcare interventions remains uncommon and process data are often poorly integrated with trial results. ${ }^{8}$ As most trials tend to focus solely on outcomes, recent studies emphasise the importance of embedding a process evaluation in controlled trials of complex interventions. ${ }^{9,10}$ The Medical Research Council (MRC) defines complex interventions as:

“...interventions with several interacting components (...) they present a number of special problems for evaluators. (...) Many of the extra problems relate to the difficulty of standardising the design and delivery of the interventions, their sensitivity to features of the local context, the organisational and logistical difficulty of applying experimental methods to service or policy changes, and the length and complexity of the causal chains linking intervention with outcome." ${ }^{11}$

The integration of outcome and process data contributes to a more thorough understanding of how the intervention functions in the intended context, allowing better 
judgment of transferability of research results into practice. ${ }^{2,12,13}$ In other words, it defines how the intervention should be implemented in comparable settings to ensure similar outcomes are reached. ${ }^{2}$ Moreover, combined outcome and process data may also reveal minimally required elements for optimal performance of the intervention in other, less comparable settings.

This paper presents the process evaluation plan of the [G]OLD-study ('Getting OLD the healthy way'), a cluster quasi-experimental trial conducted in the Netherlands which aims to evaluate whether a preventive home visitation programme for potentially frail community-dwelling older people (aged $\geq 75$ years) within general practice will lead to improved health-related quality of life and reduced disability. The delivery of care for older people within primary care is redesigned by applying components of the Chronic Care Model (CCM) ${ }^{14}$ and the Guided Care Model. ${ }^{15}$ This resulted in the [G]OLD-protocol: a patient-centred home visitation programme consisting of a comprehensive multidimensional geriatric assessment of older people's health and wellbeing, followed by, if necessary, individualised care (referral to appropriate care and/or well-being facilities) and follow-up. Figure 3.1 presents the logic model of the [G]OLD home visitation programme, developed according to the four properties of logic models as claimed by Conrad and co-workers. ${ }^{16}$ It delineates the essential components of the home visitation programme and how the theory underlying the intervention, the intervention components, and expected outcomes are linked to one another. In this way, the logic model serves as a framework for defining important intervention aspects that should be considered in the process evaluation. ${ }^{16}$

Baranowski and Stables ${ }^{17}$ argue, among others, for more thoroughly conducted process evaluations and greater consistency in concepts used to structure process evaluations. Therefore, we designed the process evaluation according to the steps for developing a process evaluation plan as suggested by Saunders and colleagues. ${ }^{18}$ They propose seven key elements of process evaluation, adapted from Baranowski and Stables ${ }^{17}$ and Linnan and Steckler, ${ }^{1}$ including fidelity (quality of implementation), dose delivered (completeness), dose received (exposure), dose received (satisfaction), reach (participation rate), recruitment, and context. We are particularly interested in the process of delivering and implementing the home visitation programme within a dynamic and complex primary care setting and therefore, we pay special attention to the element 'context' using the Normalisation Process Model. ${ }^{19}$ This model provides a conceptual framework for understanding the factors that affect the success or failure of a complex intervention within primary care in relation to the workability and integration of the intervention components. It includes four domains: (1) how work is enacted by the people doing it (interactional workability); (2) how work is understood within the networks of people around it (relational integration); (3) the place of work in a division of labour (skill-set workability); and (4) the organisational sponsorship and 
control of work (contextual integration). The model states that when a complex intervention is workable and integrated, it is more likely to become routinely embedded within everyday practice ('disposed to normalisation'). ${ }^{19,20}$ In this way, the model aids in the identification of contextual factors specific for primary care that may affect programme implementation or programme outcomes. For examples how this model can be applied, see May and colleagues. ${ }^{20}$

Population \& Environment

\begin{tabular}{|l|}
\hline Patient population \\
Community-dwelling \\
older people (aged $\geq 75$ \\
years). Exclusion \\
criteria: on waiting list for \\
a nursing home/ home \\
for the elderly, under \\
close medical supervi- \\
sion (e.g., chemothera- \\
py), and terminally ill
\end{tabular}

Environmental context Ageing of the population in the Netherlands $(7.0 \%$ $\geq 75$ yrs), especially in the regions MaastrichtHeuvelland $(8.5 \% \geq 75$ yrs) and Parkstad (8.7\% $\geq 75$ yrs).

General practices are geographically close to older people; know the patient's medical history, and have access to multidisciplinary health and well-being facilities in the neighbourhood.

Programme resources - Practice nurse (PN) with sufficient time for care for older people - General practitioner (GP) who supports PN - Adequate funding of costs of care for older people delivered by GPs
Theory \& Assumptions

Concept of problem

- Older people often suffer

from multiple and complex

health complaints $\rightarrow$ Early

identification of health

risks can help maintain

independent living

- Need for proactive,

coherent, multidisciplinary

preventive care using a

patient-centred approach

Concept of intervention

Early identification of

(potentially) frail older

people within general

practice $\rightarrow$ Redesigning

care delivery by general

practitioner and practice

nurse:

- Delivery system design:

delivering proactive

instead of reactive care

- Community resources:

establishing linkages with

(local) care and well-being

services

- Decision support:

referral based on cut-off

points in geriatric

assessment; availability of

a service map

- Clinical information

systems: system for

registration of the results

of the geriatric

assessment, planning

care and follow-up
Intervention

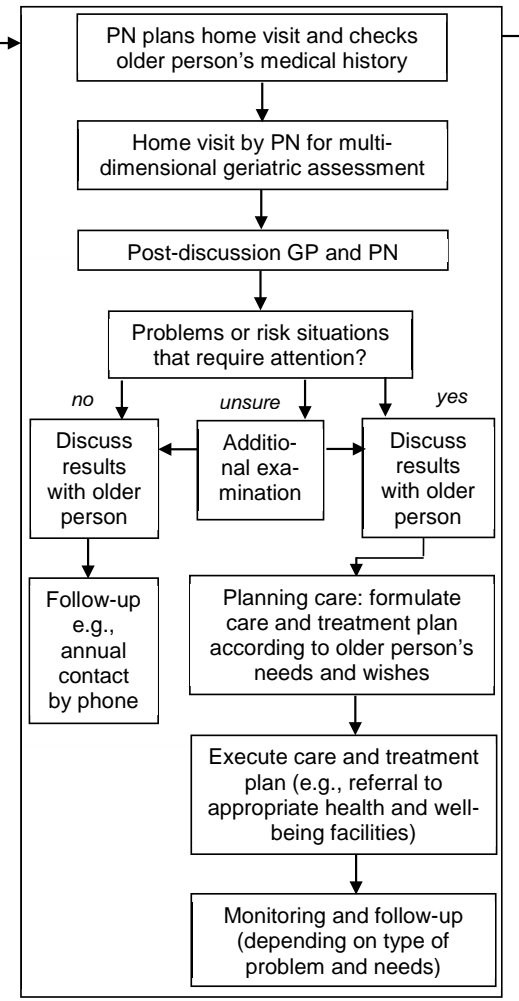

Outcomes

Main goal:

Maintain

independent

living among

community-

dwelling older

people (aged $\geq$

75 years)

Primary

outcomes:

- Improved

health-related

quality of life

- Reduced

disability in

(instrumental)

activities of daily

living

Secondary

outcomes:

- Improved

attitude towards

ageing

- Decreased

healthcare

utilisation

- Decrease in

number of

admissions to

nursing home or

home for the

elderly

- Reduced

mortality

Figure 3.1 Logic model [G]OLD preventive home visitation programme

The different components and domains mentioned above, together with the logic model of the home visitation programme, serve as a starting point for the development of the process evaluation plan for the [G]OLD-study. Overall, the current process evaluation aims to (1) monitor and assess the implementation of the home visitation programme within general practice and (2) generate findings that aid in the interpretation and explanation of the programme effects obtained in the parallel controlled trial. 


\section{METHOD}

\section{Process evaluation design}

The process evaluation is conducted parallel to the longitudinal, quasi-experimental study examining the effects of the home visitation programme and data collection is structured using a triangulation design model. ${ }^{21}$ Some results of the process evaluation are used to inform the implementation process and to perform midcourse corrections when fidelity of implementation is threatened (formative purposes). However, most process data will not be available until after completion of the implementation of the programme (summative purposes). Process data will be analysed and interpreted before the outcomes of the effect study. In this way, hypotheses may be generated that can subsequently be statistically tested. ${ }^{10}$ For instance, if the process evaluation shows variability between general practices in the quality of implementing the home visitation, how is this related to trial outcomes?

Process evaluation data are collected from all participating general practices who implement the home visitation programme, as we expect that practice characteristics (e.g., number of professionals working within general practice) and factors related to the neighbourhood in which practices are located, may influence the implementation process. For example, close proximity of other primary care professionals may facilitate collaboration in organising and/or delivering appropriate care to older people. ${ }^{22}$ Moreover, the number and type of health problems detected during the multidimensional assessment may depend on socio-economic circumstances of older people as these appear to be associated with health outcomes. ${ }^{23}$

The protocol for the [G]OLD-study was judged by the Medical Ethical Committee (MEC) of the Maastricht University Medical Centre (MUMC+) as not needing formal ethical approval. Nonetheless, the MEC approved the study protocol for the [G]OLDtrial and accompanying process evaluation.

\section{Setting}

Thirteen general practices offer proactive care to people aged 75 or older according to the home visitation programme (intervention group), whereas 11 general practices provide usual care (control group). Usual care encompasses reactive care to older people who present themselves with health problems or complaints. During the trial, control practices should not be engaged in any form of proactively assessing (potentially) frail older people's health status followed by targeted treatment or care and follow-up within the continuum of care. 
Participating intervention practices are located in the regions Maastricht-Heuvelland and Parkstad in the south of the province Limburg in the Netherlands, were ageing of the population is especially pronounced. In 2007, the Dutch College of General Practitioners stated that the responsibility for preventive care for older people should be assigned to general practitioners (GPs), supported by a practice nurse (PN). ${ }^{24}$ In the Netherlands, practice nurses substitute GPs in chronic disease management by providing screening, treatment, care and education to patients suffering from for example diabetes mellitus, chronic obstructive pulmonary disease (COPD), and those at risk for vascular diseases. Nurses possess complementary expertise compared to GPs (e.g., in health education) ${ }^{25}$ and when appropriately trained, they can achieve equally high or even better quality of care and positive health outcomes in patients as GPs. ${ }^{26,27}$ Thus, it is assumed worthwhile to delegate care for older people to PNs. In the [G]OLD-study, the $\mathrm{PN}$, supervised by the GP, is therefore in charge of conducting the home visits and reorganising care for older people. Before the start of the trial, all 13 PNs from the intervention practices participated in a two-day training to provide them with the necessary knowledge and skills for executing the different aspects of the home visitation programme.

\section{Programme implementation as planned}

The steps of the [G]OLD-protocol are illustrated in the flowchart in Figure 3.1 (see 'Intervention'). First, the PN contacts eligible older people to schedule a date for the home visit. The PN prints relevant information from the GP's information system concerning medical history and medication use to prepare for the home visit. The next step is visiting the older person at home and performing a comprehensive geriatric assessment of the person's physical, psychological, mental and social functioning using the so-called [G]OLD-instrument. This multidimensional instrument is specifically developed to assist PNs in uncovering (early signs of) health and/or well-being problems or complaints that may now or ultimately be detrimental for maintaining independent living. ${ }^{28}$ After the home visit, the PN discusses the results of the geriatric assessment with the GP. If the older person is doing completely fine and no follow-up actions are needed, the PN communicates this to the older person and checks his/her health status again after one year (either by phone or by means of a re-assessment with the [G]OLD-instrument). However, if there are any (health) problems or risk situations that require attention, the $\mathrm{PN}$ formulates a care and treatment plan, if necessary with help of the GP and in agreement with the older person's needs and wishes. The PN subsequently executes the care and treatment plan, for example by referring the older person to appropriate health and/or well-being facilities in the neighbourhood. The PN monitors progress and coordinates care and follow-up; the number of follow-up con- 
tacts depends on the type of problem and the older person's needs and wishes. Sometimes, the PN and GP may be uncertain about the presence of health problems or risk situations. An additional examination might be necessary to confirm or reject previous assumptions before a care and treatment plan is formulated. To guarantee adequate delivery of the home visitation programme, no steps are allowed to be omitted.

In addition to the steps of the intervention protocol, we propose that redesigning healthcare for older people within general practices can only be successfully accomplished if four aspects are realised. ${ }^{14}$ General practices should be prepared to change their mindset from delivering reactive care towards offering proactive care (delivery system design). Although the home visitation programme may be applied to a diverse patient population, it is specifically designed to identify health problems among the apparently healthy older people at an early stage. Furthermore, general practices should establish linkages with other professionals or organisations offering (local) care and/or well-being services to older people (community resources). In particular, the organisation of multidisciplinary meetings between different professionals is strongly recommended to effectively organise care for older people with complex and/or multiple health problems. Third, general practices should be appropriately equipped with decision-aids to help them decide about the presence or absence of health problems and, subsequently, where older people should be referred to within the range of health and well-being services available (decision support). Therefore, cut-off points are incorporated in the [G]OLD-instrument per test or question and a service map is developed for different municipalities providing an overview of care or well-being facilities available for specific health or well-being problems. Finally, to provide continuity in care for older people, a system should be developed within existing clinical information systems for general practices to register the results of the geriatric assessment, and to plan and monitor care and follow-up (clinical information systems).

\section{Process evaluation questions}

For each component of the process evaluation, several process evaluation questions are formulated (Table 3.1). Since it is not advisable nor practically feasible to measure every programme element into extensive detail, ${ }^{1}$ the questions focus on the elements of the home visitation programme that are minimally required for acceptable programme delivery (see logic model, Figure 3.1). Furthermore, in prioritising process evaluation questions, the workload or burden imposed on those responsible for collecting the data was also taken into consideration. 
Table 3.1 Process evaluation questions

Fidelity

(quality of

implementation)
Dose delivered

(completeness)

Dose received

(exposure)

Dose received

(satisfaction)

Reach

(participation rate)

Recruitment

Context

(in general)

Interactional

workability

Relational integration Do PNs have sufficient knowledge, expertise and skills to perform the activities as part of the home visitation programme?

To what extent do PNs feel confident that they can assess and address older people's (complex) health problems?

Skill-set workability Is the division of work between GP and PN acceptable?

Contextual integration Does the home visitation programme for older people fit within the range of healthcare services offered by general practices?

Are sufficient resources (e.g., time, staff, and money) available for the adequate performance of the home visitation programme by general practices? 


\section{Data collection}

Multiple data collection strategies are used to gather process data. Quantitative and qualitative process data are collected concurrently and the findings will be integrated (triangulation) to attain a complete understanding of the quality and process of implementation. $^{21,29}$ The choices for the data collection tools are based on the nature of the process evaluation questions, as well as on how the most valid and reliable information can be obtained in an efficient and least burdensome way for those involved. Process data are gathered using the following methods or tools:

1. Individual semi-structured interviews with GPs, PNs and older people. Also, short semi-structured interview by phone with GPs from control practices.

2. Feedback meetings with PNs.

3. Structured registration forms, filled-out by PNs: (1) registration of the results of the multidimensional geriatric assessment in the [G]OLD-instrument; (2) form to write down the results of the post-discussion with the GP; (3) care and treatment plan; (4) registration of number of follow-up contacts (derived from GP Information System), compliance of older people with recommendations formulated in the care and treatment plan, and extent to which older people benefited from the home visitation programme.

4. Evaluation forms for training, filled-out by PNs.

5. [G]OLD care diary for older people.

6. Continuous registration and notes by the research team.

An overview of the data collection tools/procedures and data sources per process evaluation component and accompanying process evaluation question(s) is provided in Table 3.2. The GPs and PNs of participating general practices are the most important sources from which process data are collected, as they are the key actors within the home visitation programme.

\section{Semi-structured interviews}

Semi-structured interviews were carried out with all PNs $(n=13)$ individually from participating general practices in the intervention group. PNs performed the home visits among older people consecutively within 13 months' time (estimated time required for visiting older people enrolled in the research project, given the available time of PNs for care for older people). During this intervention period, each PN was interviewed three times: after three months, six months, and at the end of the intervention period. Questions asked differed between the interview rounds and interviews lasted approximately 30 to 60 minutes. The first interview round focused on the initial implementation of the different steps of the [G]OLD home visitation protocol and experiences of PNs thus far. In the second interview, progress compared to the previous 
interview and collaboration with GPs and other healthcare providers was discussed. Finally, the last interview round again evaluated progress, as well as results achieved within general practice by implementing the home visitation programme and future utilisation of the programme after the intervention period. The various interview rounds enable detecting interim problems threatening the fidelity of implementation.

At the end of the intervention period, one GP from each participating general practice was interviewed (duration approximately 30 minutes). Using a semistructured format, GPs were asked about their experiences regarding implementation of the home visitation programme within their practice. In general practices with more than one GP, the one who most closely collaborated with the PN in implementing the home visitation programme was approached for the interview. If conflicting opinions exist between GPs within a practice regarding the programme, the other GP(s) was/were interviewed as well. Interviews with GPs and PNs took place within the GP's practice. Additionally, GPs in the control group (one per general practice) are interviewed by phone at the end of the follow-up period (18 months after baseline). A semi-structured format is used to determine the extent to which control participants might have received structured, proactive care similar to the home visitation programme (contamination).

Finally, older people were invited to share their experiences and views with respect to the home visit by the PN and subsequent follow-up actions (if applicable). Semi-structured interviews were conducted of approximately 30 minutes in older people's homes. The home setting is preferred to make older people feel at ease, which may result in richer descriptions of their experiences and opinions. The interview was conducted between three to five weeks after the home visit to make sure any followup actions (if applicable) are communicated to the older person and older people still remember details of the visit. Those cognitively impaired, as judged by the PN, were excluded from participation in the interviews. While sampling older people, strong heterogeneity among older people should be kept in mind $^{30}$ and therefore older people were purposefully sampled based on the principle of maximum variation. The variables gender, age, household status (living alone vs. living together), and health status were deemed important characteristics to consider when recruiting older people for the interviews to obtain a sample representative for those enrolled in the research project. By including a broad group of older people, we also attempted to circumvent the problem that older people tend to provide less detailed descriptions of their situation or experiences. ${ }^{31}$ From each PN, one older person (or two if the PN assessed the health status of the partner as well) he or she visited was selected for an interview. After this initial interview round, data saturation was reached.

One member of the research team (MS) was responsible for conducting all semistructured interviews. 
Table 3.2 Data collection method and data source per process evaluation component and process evaluation question

\begin{tabular}{|c|c|c|}
\hline $\begin{array}{l}\text { Process evaluation } \\
\text { component }\end{array}$ & Process evaluation question* & Data collection tool(s) / procedure(s) and data source(s) \\
\hline $\begin{array}{l}\text { Fidelity } \\
\text { (quality of } \\
\text { implementation) }\end{array}$ & $\begin{array}{l}\text { Implementation as planned }{ }^{(1)} \\
\text { redesigning care }^{(2)}\end{array}$ & $\begin{array}{l}\text { Semi-structured interviews with GPs and PNs; feedback sessions } \\
\text { with PNs; structured registration forms filled-out by PN }\end{array}$ \\
\hline $\begin{array}{l}\text { Dose delivered } \\
\text { (completeness) }\end{array}$ & $\begin{array}{l}\text { Steps [G]OLD-protocol followed } \\
\text { by PN }{ }^{(3)}\end{array}$ & $\begin{array}{l}\text { Semi-structured interviews with PNs; feedback sessions with } \\
\text { PNs; structured registration forms filled-out by PN; semi- } \\
\text { structured interviews with older people }\end{array}$ \\
\hline $\begin{array}{l}\text { Dose received } \\
\text { (exposure) }\end{array}$ & $\begin{array}{l}\text { Compliance older people to } \\
\text { follow-up actions }{ }^{(4)}\end{array}$ & $\begin{array}{l}\text { Registration of compliance per older person according to opinion } \\
\text { of PN on structured form; [G]OLD care diary for older people }\end{array}$ \\
\hline \multirow[t]{3}{*}{$\begin{array}{l}\text { Dose received } \\
\text { (satisfaction) }\end{array}$} & Satisfaction GP+PN ${ }^{(5)}$ & $\begin{array}{l}\text { In-depth questions during semi-structured interviews with GPs } \\
\text { and PNs }\end{array}$ \\
\hline & Satisfaction older people ${ }^{(6)}$ & $\begin{array}{l}\text { In-depth questions during semi-structured interviews with older } \\
\text { people }\end{array}$ \\
\hline & Benefit older people ${ }^{(7)}$ & Benefit judged by PN and registered on structured form \\
\hline \multirow{5}{*}{$\begin{array}{l}\text { Reach } \\
\text { (participation } \\
\text { rate) }\end{array}$} & Number of participants ${ }^{(8)}$ & $\begin{array}{l}\text { Registration no. of participants and non-participants in trial } \\
\text { database }\end{array}$ \\
\hline & Reasons non-participation ${ }^{(9)}$ & $\begin{array}{l}\text { Reminder non-responders (by phone) and notes research team } \\
\text { reasons for non-participation }\end{array}$ \\
\hline & Opinion reach GP+PN ${ }^{(10)}$ & $\begin{array}{l}\text { Semi-structured interviews GPs and PNs; feedback sessions with } \\
\text { PNs }\end{array}$ \\
\hline & Completion steps protocol ${ }^{(11)}$ & $\begin{array}{l}\text { Structured registration forms filled-out by PN; registration no. of } \\
\text { drop-outs in trial database }\end{array}$ \\
\hline & Reasons drop-out ${ }^{(12)}$ & Notes research team reasons for drop-out \\
\hline Recruitment & Recruitment procedures ${ }^{(13)}$ & Narrative report by research team of recruitment activities \\
\hline \multirow[t]{2}{*}{$\begin{array}{l}\text { Context } \\
\text { (in general) }\end{array}$} & Implementation issues ${ }^{(14)}$ & $\begin{array}{l}\text { Semi-structured interviews with GPs and PNs; notes research } \\
\text { team }\end{array}$ \\
\hline & Contamination ${ }^{(15)}$ & $\begin{array}{l}\text { Short semi-structured interview by phone with GPs participating } \\
\text { in the control group }\end{array}$ \\
\hline $\begin{array}{l}\text { Interactional } \\
\text { workability }\end{array}$ & $\begin{array}{l}\text { Congruence PN and GP, and PN } \\
\text { and older people }\end{array}$ & Semi-structured interviews with PNs \\
\hline \multirow[t]{2}{*}{$\begin{array}{l}\text { Relational } \\
\text { integration }\end{array}$} & Expertise $P N^{(17)}$ & $\begin{array}{l}\text { Semi-structured interviews with GPs, PNs, and older people; } \\
\text { evaluation form training PN }\end{array}$ \\
\hline & Confidence PN ${ }^{(18)}$ & Semi-structured interviews with PNs; evaluation form training PN \\
\hline Skill-set workability & Division of work GP+PN ${ }^{(19)}$ & Semi-structured interviews with GPs and PNs \\
\hline Contextual & Fit within care services ${ }^{(20)}$ & Semi-structured interviews with GPs and PNs \\
\hline integration & Resources available ${ }^{(21)}$ & Semi-structured interviews with GPs and PNs \\
\hline
\end{tabular}

\footnotetext{
${ }^{*}$ Bracketed numbers correspond to the numbers of the process evaluation questions presented in Table 3.1.
} 


\section{Feedback meetings}

Twice during the intervention period, a feedback meeting was organised for all participating PNs. During these meetings, PNs were informed about the progress of the research project and other research-related aspects, such as the logistic issues concerning collecting (process) data and returning data to the research team. Interim results of the individual semi-structured interviews with PNs were fed back to the whole group and, if necessary, corrections to programme implementation were made to guarantee the programme is delivered with fidelity. PNs were offered the possibility to interact with other PNs and discuss the interim results. A research assistant made notes of the discussion for evaluation purposes.

\section{Structured registration forms}

The [G]OLD-instrument, including the registration form for the post-discussion with the GP, and the care and treatment plan were distributed to PNs during the training sessions before the onset of the intervention period. PNs were requested to return the forms per older person stepwise during the intervention period after the care and treatment plan is formulated. Upon receipt, a research assistant checked the documents on completeness and accuracy of reporting, thereby serving as a proxy for complete and acceptable delivery of the first steps of the [G]OLD intervention protocol by PNs. Any issues discovered that threatened implementation fidelity, were discussed with PNs in person or during a feedback meeting. At the end of the follow-up period (18 months after baseline), which differed per older person since the home visits were conducted consecutively by PNs during 13 months, PNs were asked to register in a structured document for each older person the number of follow-up contacts, compliance to recommendations, and extent to which the older person benefited from the home visitation programme. Although the latter two aspects were based on the PN's judgement, we believe PNs had little reason to give social-desirable answers.

PNs documented in the [G]OLD-instrument the results of the multidimensional assessment (answers given by older people to tests and questions), as well as more process-related information such as the duration of the visit and whether other people were present during the assessment. In the structured form for the post-discussion with the GP, PNs could check from a list which problems, complaints or issues had been discussed with the GP, what the main findings were, and which follow-up actions would be undertaken (e.g., additional examination, treatment, referral). The PN formulated a care and treatment plan in agreement with the older person's wishes that contained details per detected problem or complaint regarding the goals to be achieved, who will take action (divided into actions by general practice and actions by other professionals within the continuum of care), and when the problem will be evaluated. A case-manager was assigned who is responsible for coordinating care and 
monitoring over time and PNs were required to formulate an overall goal to be reached (e.g., improvement of functioning, maintaining current level of functioning).

\section{Evaluation forms}

At the end of each training session (two days in total), PNs were requested to fill-out an evaluation form. For both training sessions the same structured format was used, including open-ended concerning their satisfaction with the training in general and close-ended questions evaluating their opinion concerning knowledge gained and skills acquired, extent to which they felt prepared to conduct the home visits, and the need for additional training.

\section{[G]OLD care diary}

Older people received the [G]OLD care diary before the home visit, together with the baseline questionnaire as part of the effect study. They were asked to register details of their contacts with healthcare and/or well-being providers (date of contact, name of professional or organisation, reason for contact, a grade representing satisfaction with contact, etc.) during 18-months follow-up. For process evaluation purposes, this information is used as an indicator of compliance to recommended follow-up actions according to the care and treatment plan. At 18-months, older people are requested to send back the care diary together with the final follow-up questionnaire of the effect study.

\section{Continuous registration}

During the recruitment and intervention period, members of the research team provided narrative descriptions of procedures applied during the recruitment process and factors influencing the implementation of the home visitation programme mentioned outside the semi-structured interviews with GPs and PNs. Furthermore, nonparticipation and drop-out rates, as well as the reasons for non-participation and dropout (if mentioned by older people or the PN) were documented during the recruitment phase, and the intervention and follow-up phase, respectively.

\section{Data analysis}

Quantitative process data will be analysed using the software package SPSS for Windows, version 17.0. Descriptive statistics will be computed (e.g., means, frequencies) for quantitative variables, such as the amount of non-participants and drop-outs, follow-up contacts after the home visit, compliance of older people with follow-up actions as judged by the PN, and number and duration of home visits conducted by PNs. 
All individual semi-structured interviews are digitally recorded (except for the telephone contact with GPs from control practices; in this case, notes are made), after obtaining verbal consent from participants, and transcribed verbatim. The analysis process is supported by the software package NVivo 7, in which transcripts are anonymously transferred. The researcher (MMNS) will code the transcripts and another member of the research team will code one quarter of the transcripts. A general inductive approach ${ }^{32}$ will be applied in which the analysis is largely guided by the process evaluation objectives. Systematic and rigorous reading and coding of the transcripts will allow major themes to emerge. Other qualitative data will be assessed using conventional content analysis to allow themes to emerge from the data. ${ }^{33}$ This will be applied to, for example, the open-ended questions included in the evaluation forms of the training for PNs, and the notes made by the research team of the feedback sessions with PNs. Descriptions of procedures applied will be summarised in a narrative report.

\section{DISCUSSION}

This paper presents into detail the development of a theory-based process evaluation plan for the [G]OLD home visitation programme aimed at identifying potentially frail community-dwelling older people (aged $\geq 75$ years) within general practice. When combined, the findings from the effect study and process evaluation inform the potential implementation of the [G]OLD-home visitation programme on a larger scale.

In general, studies describing into detail the rationale for conducting a process evaluation alongside a clinical trial and the methods chosen to gather process data are relatively rare. In agreement with Ellard and colleagues, ${ }^{34}$ we believe that if process evaluations are funded and undertaken, both the process evaluation plans as well as process evaluation findings should be published more often, particularly with respect to complex, patient-centred healthcare interventions. The insights gained are imperative for the development of evidence-based interventions that are likely to be sustained when implemented in the intended context. Moreover, it is essential to develop a process evaluation plan prospectively to follow the implementation of a complex intervention from its initial use until continued use (for examples of prospective process evaluation plans, see Grimshaw and co-workers ${ }^{35}$; Ellard and co-workers ${ }^{34}$ ). A prospective plan prevents evaluators from collecting unnecessary data and additionally helps to plan the collection of process data in an efficient way (i.e., using data collection tools that can be used for various purposes). ${ }^{36}$

With respect to home visitation programmes for (frail) older people, only few studies so far incorporated a detailed process evaluation alongside the main trial (see 
for example Alessi and colleagues, ${ }^{37}$ Van Haastregt and colleagues, ${ }^{38}$ and NicolaidesBouman and colleagues ${ }^{39}$ ). Although many more home visitation programmes have been developed thus far, their effectiveness remains a controversial issue. ${ }^{40}$ Trials in this field may benefit from a thorough process evaluation to determine, among others, the factors that facilitate or hinder adequate implementation of the intervention. This information can assist in clarifying favourable or unfavourable intervention effects, thereby providing insight into the contradictory results obtained so far. Especially since the current home visitation programme is fully embedded within primary care practices, we consider a process evaluation to be of utmost importance to assess the implementation and routinisation process within a complex and dynamic context into detail.

Process evaluation may serve many purposes, depending on the main objectives of the evaluation, available resources, the nature of the intervention, and the setting in which it is implemented. The present process evaluation plan may serve as an example for other evaluators aiming to incorporate a comprehensive process evaluation in a trial of a complex, patient-centred healthcare intervention. Although part of the theoretical framework underlying the process evaluation plan originally guides the development of a process evaluation for health promotion interventions, ${ }^{18}$ we believe the described components and steps are also applicable to patient-centred interventions implemented in primary care, particularly when combined with the more detailed contextual factors derived from the Normalisation Process Model. ${ }^{19,20}$ There are many different variables that influence normalisation or routinisation (see Rogers ${ }^{41}$ ), but this model specifically addresses factors related to the primary care setting. Nevertheless, a challenge we faced during the design of the process evaluation was deciding which aspects of the complex intervention to evaluate. The logic model we developed contributed in the decision-making process, as it helped to focus on the most important aspects of the content and delivery of the home visitation programme within general practice. In addition, a challenge while executing the process evaluation is the use of formative process data. We need to consider to what extent interim adjustments or changes to the intervention protocol can be made to ensure the home visitation programme is implemented with fidelity, without threatening the integrity of the parallel controlled trial. 


\section{REFERENCES}

1. Linnan L, Steckler A. Process evaluation for public health interventions and research. An overview. In: Steckler A, Linnan L, editors. Process evaluation for public health interventions and research. San Francisco: Jossey-Bass; 2002. p. 1-24.

2. Harachi TW, Abbott RD, Catalano RF, Haggerty KP, Fleming CB. Opening the black box: using process evaluation measures to assess implementation and theory building. Am J Community Psychol 1999;27:711-731.

3. Rychetnik L, Frommer M, Hawe $P$, Shiell A. Criteria for evaluating evidence on public health interventions. J Epidemiol Community Health 2002;56:119-127.

4. Flottorp S, Håvelsrud K, Oxman AD. Process evaluation of a cluster randomized trial of tailored interventions to implement guidelines in primary care. Why is it so hard to change practice? Fam Pract 2003;20:333-339.

5. McKenna HP, Ashton S, Keeney S. Barriers to evidence-based practice in primary care. J Adv Nurs 2004;45:178-189.

6. Grol R. Successes and failures in the implementation of evidence-based guidelines for clinical practice. Med Care 2001;39:II-46-II-54.

7. Bosch M, Van der Weijden T, Wensing M, Grol R. Tailoring quality improvement interventions to identified barriers: a multiple case analysis. J Eval Clin Pract 2007;13:161-168.

8. Lewin S, Glenton C, Oxman AD. Use of qualitative methods alongside randomised controlled trials of complex healthcare interventions: methodological study. Br Med J 2009;339:b3496.

9. Craig P, Dieppe P, Macintyre S, Michie S, Nazareth I, Petticrew M. Developing and evaluating complex interventions: the new Medical Research Council guidance. Br Med J 2008;337:a1655.

10. Oakley A, Strange V, Bonell C, Allen E, Stephenson J, RIPPLE Study Team. Process evaluation in randomised controlled trials of complex interventions. Br Med J 2006;332:413-416.

11. Medical Research Council. Developing and evaluating complex interventions: new guidance. London: MRC, 2008.

12. Glasgow RE, Emmons KM. How can we increase translation of research into practice? Types of evidence needed. Annu Rev Public Health 2007;28:413-433.

13. Bradley F, Wiles R, Kinmonth A-L, Mant D, Gantley M. Development and evaluation of complex interventions in health services research: case study of the Southampton heart integrated care project (SHIP). Br Med J 1999;318:711-715.

14. Bodenheimer T, Wagner EH, Grumbach K. Improving primary care for patients with chronic illness. J Am Med Assoc 2002;288:1775-1779.

15. Boult C, Karm L, Groves C. Improving chronic care: the "Guided Care" model. Perm J 2008;12:50-54.

16. Conrad KJ, Randolph FL, Kirby MW, Bebout RR. Creating and using logic models: four perspectives. Alcohol Treat Q 1999;17:17-31.

17. Baranowski T, Stables G. Process evaluations of the 5-a-day projects. Health Educ Behav 2000;27:157166.

18. Saunders RP, Evans MH, Joshi P. Developing a process-evaluation plan for assessing health promotion program implementation: a how-to guide. Health Promot Pract 2005;6:134-147.

19. May CR. A rational model for assessing and evaluating complex interventions in health care. BMC Health Serv Res 2006;6:86.

20. May CR, Mair FS, Dowrick CF, Finch TL. Process evaluation for complex interventions in primary care: understanding trials using the normalization process model. BMC Fam Pract 2007;8:42.

21. Creswell JW, Fetters MD, Ivankova NV. Designing a mixed methods study in primary care. Ann Fam Med 2004;2:7-12. 
22. Lemieux-Charles L, McGuire WL. What do we know about health care team effectiveness? A review of the literature. Med Care Res Rev 2006;63:263-300.

23. Grundy E, Sloggett A. Health inequalities in the older population: the role of personal capital, social resources and socio-economic circumstances. Soc Sci Med 2003;56:935-947.

24. Dutch College of General Practitioners. NHG-Standpunt Toekomstvisie Huisartsenzorg. Huisartsgeneeskunde voor ouderen [Statement of the NHG, Future of General Practitioner Care. General practice medicine for older people]. Utrecht: NHG, 2007.

25. Dierick-van Daele ATM, Spreeuwenberg C, Derckx EWCC, Van Leeuwen Y, Toemen T, Legius M, et al. The value of nurse practitioners in Dutch general practices Qual Prim Care 2010;18:231-241.

26. Laurant M, Reeves D, Hermens R, Braspenning J, Grol R, Sibbald B. Substitution of doctors by nurses in primary care. Cochrane Database Syst Rev 2004;4:Art. No.: CD001271.

27. Voogdt-Pruis HR, Beusmans GHMI, Gorgels APM, Kester ADM, Van Ree JW. Effectiveness of nursedelivered cardiovascular risk management in primary care: a randomised trial. $\mathrm{Br} J$ Gen Pract 2010;60:40-46.

28. Stijnen MMN, Jansen MWJ, Vrijhoef HJM, Duimel-Peeters IGP. Development of a home visitation programme for the early detection of health problems in potentially frail community-dwelling older people by general practices. Eur J Ageing 2013;10:49-60.

29. Tashakkori A, Teddlie C. Mixed methodology: combining qualitative and quantitative approaches. Thousand Oaks, Calif.: Sage Publications; 1998.

30. Wenger GC. Interviewing older people. In: Gubrium JF, Holstein JA, editors. Handbook of interview research: context \& method. Thousand Oaks, Calif.: Sage Publications; 2001. p. 259-278.

31. Kirkevold $M$, Bergland $A$. The quality of qualitative data: issues to consider when interviewing participants who have difficulties providing detailed accounts of their experiences. Int J Qual Stud Health Well-being 2007;2:68-75.

32. Thomas DR. A general inductive approach for analyzing qualitative evaluation data. Am J Eval 2006;27:237-246.

33. Hsieh H, Shannon SE. Three approaches to qualitative content analysis. Qual Health Res 2005;15:12771288.

34. Ellard DR, Taylor SJC, Parsons S, Thorogood M. The OPERA trial: a protocol for the process evaluation of a randomised trial of an exercise intervention for older people in residential and nursing accommodation. Trials 2011;12:28.

35. Grimshaw JM, Zwarenstein M, Tetroe JM, Godin G, Graham ID, Lemyre L, et al. Looking inside the black box: a theory-based process evaluation alongside a randomised controlled trial of printed educational materials (the Ontario printed educational message, OPEM) to improve referral and prescribing practices in primary care in Ontario, Canada. Implement Sci 2007;2:38.

36. Helitzer D, Yoon S. Process evaluation of the adolescent social action program in New Mexico. In: Steckler A, Linnan L, editors. Process evaluation for public health interventions and research. San Francisco: Jossey-Bass; 2002. p. 83-113.

37. Alessi CA, Stuck AE, Aronow HU, Yuhas KE, Bula CJ, Madison R, et al. The process of care in preventive in-home comprehensive geriatric assessment. J Am Geriatr Soc 1997;45:1044-1050.

38. Van Haastregt JCM, Van Rossum E, Diederiks JPM, De Witte LP, Voorhoeve PM, Crebolder HFJM. Process-evaluation of a home visit programme to prevent falls and mobility impairments among elderly people at risk. Patient Educ Couns 2002;47:301-309.

39. Nicolaides-Bouman A, Rossum EV, Habets H, Kempen GIJM, Knipschild P. Home visiting program for older people with health problems: process evaluation. J Adv Nurs 2007;58:425-435.

40. Huss A, Stuck AE, Rubenstein LZ, Egger M, Clough-Gorr KM. Multidimensional preventive home visit programs for community-dwelling older adults: a systematic review and meta-analysis of randomized controlled trials. J Gerontol A Biol Sci Med Sci 2008;63A:298-307.

41. Rogers EM. Diffusion of innovations. 5th ed. New York: Free Press; 2003. 


\section{4}

\section{Nurse-led home visitation programme to improve health-related quality of life and reduce disability among potentially frail community-dwelling older people in general practice: a theory-based process evaluation}

Published as:

Stijnen MMN, Jansen MWJ, Duimel-Peeters IGP, Vrijhoef HJM. Nurse-led home visitation programme to improve health-related quality of life and reduce disability among potentially frail community-dwelling older people in general practice: a theory-based process evaluation. BMC Fam Pract, 2014;15:173. 


\section{ABSTRACT}

\section{Background}

Population ageing fosters new models of care delivery for older people that are increasingly integrated into existing care systems. In the Netherlands, a primary-care based preventive home visitation programme has been developed for potentially frail community-dwelling older people (aged $\geq 75$ years), consisting of a comprehensive geriatric assessment during a home visit by a practice nurse followed by targeted interdisciplinary care and follow-up over time. A theory-based process evaluation was designed to examine (1) the extent to which the home visitation programme was implemented as planned and (2) the extent to which general practices successfully redesigned their care delivery.

\section{Methods}

Using a mixed-methods approach, the focus was on fidelity (quality of implementation), dose delivered (completeness), dose received (exposure and satisfaction), reach (participation rate), recruitment, and context. Twenty-four general practices participated, of which 13 implemented the home visitation programme and 11 delivered usual care to older people. Data collection consisted of semi-structured interviews with practice nurses (PNs), general practitioners (GPs), and older people; feedback meetings with PNs; structured registration forms filled-out by PNs; and narrative descriptions of the recruitment procedures and registration of inclusion and drop-outs by members of the research team.

\section{Results}

Fidelity of implementation was acceptable, but time constraints and inadequate reach (i.e., the relatively healthy older people participated) negatively influenced complete delivery of protocol elements, such as interdisciplinary cooperation and follow-up of older people over time. The home visitation programme was judged positively by PNs, GPs, and older people. Useful tools were offered to general practices for organising proactive geriatric care.

\section{Conclusions}

The home visitation programme did not have major shortcomings in itself, but the delivery offered room for improvement. General practices successfully redesigned their care delivery from reactive towards proactive care, but perceived barriers require attention to allow for sustainability of the home visitation programme over time. 


\section{BACKGROUND}

Healthcare professionals worldwide are increasingly called upon to organise and deliver care to a growing number of older people. This stimulated the development of various multifactorial interventions and care models aimed at maintaining independent living and the prevention of disability and other adverse outcomes in communitydwelling older people. ${ }^{1-3}$ Especially primary care has been considered ideally suited to address the needs of older people, and more specifically frail older people who are at risk of functional decline and hospitalisation, predominantly due to their patientoriented focus. ${ }^{4,5}$ In several countries with a strongly developed primary healthcare system, such as the UK, Denmark and the Netherlands, interventions comprising comprehensive geriatric assessment (CGA) exist in which the general practitioner (GP) acts as the central care provider. ${ }^{6-8}$ However, primary care based models for care for older people also pose challenges to GPs. These relate to difficulties in dealing with multiple and often co-occurring medical conditions, (inter)personal challenges (e.g., communication barriers, time pressure), and the burden of administrative work. ${ }^{9}$

In the south of the Netherlands, the [G]OLD ('Getting OLD the healthy way') preventive home visitation programme was developed to improve health-related quality of life and reduce disability among potentially frail community-dwelling older people in general practice. ${ }^{10}$ Care delivery for older people within general practices is redesigned using components of the Chronic Care Model $^{11}$ and the Guided Care Model. ${ }^{12}$ This resulted in a patient-centred home visitation programme comprising a CGA of older people's health and well-being during a home visit by the practice nurse (PN). If necessary, older people are subsequently referred to individually appropriate health and/or well-being services in their neighbourhood, followed by monitoring and evaluation over time.

Due to its multi-component nature and integration in the dynamic primary care setting, the [G]OLD home visitation programme can be characterised as a complex intervention. Besides investigating the effects on patient outcomes in a large-scale controlled trial, it is equally important to obtain a profound understanding of how complex interventions function in their intended context. ${ }^{13-15}$ Therefore, we prospectively designed a process evaluation to follow the implementation of the [G]OLD home visitation programme from its initial use until continued use. ${ }^{16}$ Such pre-planned process evaluations performed alongside the effect evaluation allow for in-depth information to differentiate between interventions that have shortcomings in itself (intervention failure) and those that are badly delivered (implementation failure). ${ }^{17,18}$ Instead of merely implementing, the present home visitation programme required general practices to redesign their care delivery for potentially frail older people from reactive, disease-oriented care towards proactive, patient-oriented care. As a result, 
the objectives of the process evaluation are to examine (1) the extent to which the [G]OLD home visitation programme was implemented as planned in general practices, and (2) the extent to which general practices successfully redesigned their care delivery.

\section{METHODS / DESIGN}

\section{Process evaluation design}

The process evaluation plan was designed according to the seven key elements proposed by Saunders and colleagues, ${ }^{19}$ as adapted from Baranowski and Stables ${ }^{20}$ and Linnan and Steckler ${ }^{21}$ : fidelity (quality of implementation), dose delivered (completeness), dose received (exposure; satisfaction), reach (participation rate), recruitment, and context. The element 'context' was explored into more detail using the Normalisation Process Model ${ }^{22}$ to identify factors that affect the success or failure of delivering and implementing the intervention in a dynamic and complex primary care setting. The process evaluation questions per component of the process evaluation are summarised in Table 4.1. Further details concerning the design of the process evaluation plan are discussed elsewhere. ${ }^{16}$

The Medical Ethical Committee (MEC) of Maastricht University Medical Centre (MUMC+) judged the protocol of the [G]OLD-study and the accompanying process evaluation as not needing formal ethical approval (METC 10-4-015). Nonetheless, the MEC approved the study protocol and related documents. Written informed consent was obtained from participants at recruitment and additional verbal informed consent was obtained for the interviews on behalf of the process evaluation.

\section{Setting and participants}

The process evaluation was conducted parallel to a longitudinal, quasi-experimental trial investigating the effects of the [G]OLD home visitation programme on healthrelated quality of life and disability. ${ }^{10}$ Twenty-four general practices from three regions in the south of the Netherlands ('Maastricht-Heuvelland', 'Parkstad', and 'MiddenLimburg') were involved in the trial and all participated in the accompanying process evaluation. Thirteen general practices redesigned their care delivery and implemented the home visitation programme between July 2010 and September 2011 (intervention group), whereas 11 general practices offered usual care to older people (i.e., reactive care when older people present themselves with health problems or complaints) (control group). GPs and PNs from the intervention group were the key actors within the home visitation programme and therefore, they are the main sources from which pro- 
cess data were gathered. Thirteen PNs (one PN worked in two participating general practices; one general practice had two PNs) were responsible for implementing the home visitation programme. In several countries, including the Netherlands, PNs increasingly substitute the GP in chronic disease management and now in care for older people as well. ${ }^{23-25}$ Finally, the experiences of older people were incorporated.

Table 4.1 Process evaluation questions and data collection tools per component of the process evaluation plan

\begin{tabular}{|c|c|c|}
\hline Component & Process evaluation questions & Data collection tools \\
\hline $\begin{array}{l}\text { Fidelity } \\
\text { (quality of } \\
\text { implementation) }\end{array}$ & $\begin{array}{l}\text { To what extent were all elements of the home visitation } \\
\text { programme implemented as planned? } \\
\text { Is care delivery for older people within general practices } \\
\text { successfully redesigned? } \\
\text { Did GPs and PNs change their mindset from delivering reactive } \\
\text { care to proactive care? } \\
\text { To what extent were linkages established with other } \\
\text { professionals or organisations? } \\
\text { Did general practices receive useful decision-aids to support } \\
\text { decision-making? } \\
\text { Is a registration system realised that is (practically) useful for } \\
\text { general practices? }\end{array}$ & $\begin{array}{l}\text { Semi-structured interviews } \\
\text { GP/PN; structured registration } \\
\text { forms } \\
\end{array}$ \\
\hline $\begin{array}{l}\text { Dose delivered } \\
\text { (completeness) }\end{array}$ & $\begin{array}{l}\text { To what extent did PNs follow all steps of the intervention } \\
\text { protocol (see Figure } 4.1 \text { )? }\end{array}$ & $\begin{array}{l}\text { Semi-structured interviews GP/PN } \\
\text { and older people; structured } \\
\text { registration forms }\end{array}$ \\
\hline $\begin{array}{l}\text { Dose received } \\
\text { (exposure) }\end{array}$ & $\begin{array}{l}\text { To what extent were older people compliant with follow-up } \\
\text { actions formulated in the care and treatment plan? }\end{array}$ & Structured registration forms \\
\hline \multirow[t]{3}{*}{$\begin{array}{l}\text { Dose received } \\
\text { (satisfaction) }\end{array}$} & $\begin{array}{l}\text { To what extent were GPs and PNs satisfied with organising } \\
\text { care according to the home visitation programme? }\end{array}$ & Semi-structured interviews GP/PN \\
\hline & $\begin{array}{l}\text { To what extent were older people satisfied with the home } \\
\text { visit? }\end{array}$ & $\begin{array}{l}\text { Semi-structured interviews older } \\
\text { people }\end{array}$ \\
\hline & $\begin{array}{l}\text { To what extent did older people benefit from the home visit } \\
\text { and, if necessary, subsequent follow-up actions? }\end{array}$ & Structured registration forms \\
\hline \multirow[t]{5}{*}{$\begin{array}{l}\text { Reach } \\
\text { (participation rate) }\end{array}$} & $\begin{array}{l}\text { What proportion of the intended target population } \\
\text { participated? }\end{array}$ & Registration trial database \\
\hline & What were the reasons for non-participation of older people? & $\begin{array}{l}\text { Reminder non-responders and } \\
\text { notes in trial database }\end{array}$ \\
\hline & $\begin{array}{l}\text { Was the right target population reached according to } \\
\text { GPs/PNs? }\end{array}$ & Semi-structured interviews GP/PN \\
\hline & $\begin{array}{l}\text { What proportion of older people completed all steps of the } \\
\text { intervention protocol? }\end{array}$ & Registration trial database \\
\hline & What were the reasons for drop-out of older people enrolled? & Notes in trial database \\
\hline Recruitment & $\begin{array}{l}\text { What procedures were used to recruit general practices and } \\
\text { older people for participation? }\end{array}$ & Narrative report by project team \\
\hline
\end{tabular}




\begin{tabular}{|c|c|c|}
\hline Component & Process evaluation questions & Data collection tools \\
\hline \multirow[t]{2}{*}{ Context } & $\begin{array}{l}\text { What barriers and facilitators influenced implementation of } \\
\text { the home visitation programme within general practices? }\end{array}$ & Semi-structured interviews GP/PN \\
\hline & $\begin{array}{l}\text { To what extent did the control group receive the intervention } \\
\text { or similar types of proactive care (contamination)? }\end{array}$ & $\begin{array}{l}\text { Short semi-structured interview } \\
\text { GP by phone }\end{array}$ \\
\hline $\begin{array}{l}\text { Interactional } \\
\text { workability }\end{array}$ & $\begin{array}{l}\text { To what extent was congruence accomplished between PNs } \\
\text { and older people and GPs and PNs regarding detected (health) } \\
\text { problems and/or follow-up actions? }\end{array}$ & $\begin{array}{l}\text { Semi-structured interviews GP/PN } \\
\text { and older people }\end{array}$ \\
\hline Relational integration & $\begin{array}{l}\text { Did PNs have sufficient knowledge, expertise and skills to } \\
\text { perform the activities as part of the home visitation } \\
\text { programme? } \\
\text { To what extent did PNs feel confident that they could assess } \\
\text { and address older people's health problems? }\end{array}$ & $\begin{array}{l}\text { Semi-structured interviews GP/PN } \\
\text { and older people; evaluation form } \\
\text { during training session }\end{array}$ \\
\hline Skill-set workability & Was the division of work between GP and PN acceptable? & Semi-structured interviews GP/PN \\
\hline Contextual integration & $\begin{array}{l}\text { Did the home visitation programme fit within the range of } \\
\text { healthcare services offered by general practices? } \\
\text { Were sufficient resources (e.g., time, staff, and money) } \\
\text { available for the adequate performance of the home visitation } \\
\text { programme by general practices? }\end{array}$ & Semi-structured interviews GP/PN \\
\hline
\end{tabular}

\section{[G]OLD home visitation programme}

Figure 4.1 illustrates the steps of the [G]OLD home visitation programme that need to be undertaken by PNs, in collaboration with the GP, to ensure optimal delivery of the intervention to older people. Although PNs could adapt certain steps (i.e., how to arrange follow-up of older people over time) to older person's needs and their own working routine, no steps were allowed to be omitted. PNs used the [G]OLDinstrument, a CGA to obtain a complete overview of older people's physical, psychological, mental, and social functioning, as well as lifestyle and medication use. This instrument was specifically developed for application by PNs in general practices. ${ }^{26}$ If required, PNs could administer part two of the [G]OLD-instrument consisting of more elaborate tests concerning cognition, depression, and personality disorders. 


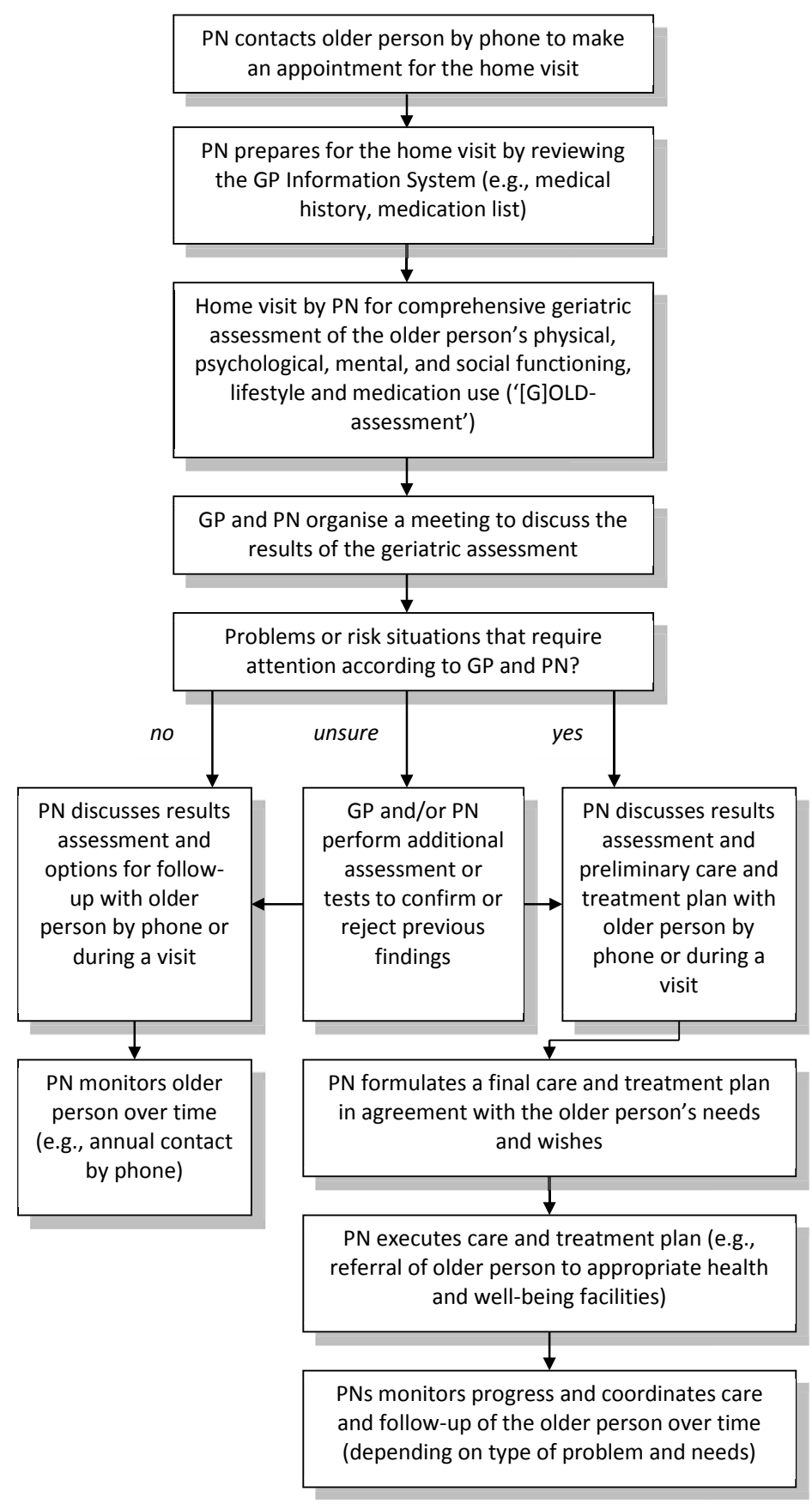

Figure 4.1 Steps of the [G]OLD home visitation programme (intervention protocol) 
Besides these steps, care delivery to older people within general practices could only be successfully redesigned if four additional aspects from the Chronic Care Model ${ }^{11}$ were realised. In line with the underlying philosophy of the intervention (i.e., identifying health and well-being problems among older people at an early stage), general practices had to change their mindset from delivering reactive care towards offering proactive care ('delivery system design'). In addition, to offer individually appropriate care to older people, interdisciplinary collaboration should be fostered by establishing linkages (e.g., multidisciplinary meetings) with (local) professionals or organisations offering care and/or well-being services to older people ('community resources'). Further, appropriate decision-aids and tools had to be delivered by the project team to assist general practices in deciding about the presence or absence of problems detected through the CGA, as well as where to refer older people with specific problems to within the available range of health and well-being services ('decision support'). Finally, the local primary care organisations had to realise an ICT-based clinical information system within existing systems in general practices to register the findings from the CGA and the results of monitoring and follow-up of older people over time ('clinical information systems').

Before the start of the intervention period in July 2010, all PNs participated in a two-day training session that focused on gaining knowledge and skills to carry out the different steps of the home visitation programme. Among others, PNs received background information on and could practice applying the [G]OLD-instrument among older people and they were brought into contact with several professionals or organisations offering care and/or well-being services to older people.

\section{Data collection}

Mixed-methods research was conducted in which quantitative and qualitative data complemented one another to yield an enriched understanding of the implementation process and redesigning care delivery by general practices. Using a convergent-parallel approach, quantitative and qualitative methods were given equal priority, data were gathered concurrently, and integration took place at the interpretation or conclusion stage of the research. This resulted in a so-called parallel-databases design ${ }^{27}$ or also called a triangulation design model, which is frequently applied in primary care research. ${ }^{28}$ An overview of the data collection tools per research question guiding the process evaluation is presented in Table 4.1.

\section{Semi-structured interviews}

Individual semi-structured interviews were conducted with PNs and GPs in the general practice and with older people in their homes. The process evaluation questions guid- 
ed the topic lists for the interviews. All 13 participating PNs were interviewed three times (i.e., after three months, six months, and at the end of the intervention period) to gain a detailed overview of their experiences in various phases of the implementation process. The interviews lasted approximately 30 to 60 minutes each.

At the end of the intervention period, one GP from each general practice participated in a 30-minute interview. In general practices with several GPs, the one who was most closely involved in implementing the home visitation programme was approached. As a result, 13 interviews were conducted with 14 GPs (in one general practice both GPs participated in the interview). At the end of the follow-up period of the trial (18 months after baseline), one GP per control practice was interviewed shortly by phone to determine the extent to which the general practice had been involved in proactive care similar to the intervention group (contamination).

After the home visit, older people were invited to share their experiences and views regarding the home visitation programme. Interviews lasted approximately 30 minutes and took place three to five weeks after the home visit. This gave PNs sufficient time to communicate the care and treatment plan to the older person, while the risk that the older person would not remember details of the home visit anymore was not yet too high. Initially, one older person (or a couple, if both received a CGA) per general practice/PN was purposefully selected (total $n=17$ older people, including 3 couples) in consultation with the PN based on the principle of maximum variation by taking into account gender, age, household status (living alone vs. living together), and health status (no/few problems detected vs. multiple problems detected). After the initial interview round, data saturation was reached as no new themes or issues arose during the coding process.

\section{Structured registration forms}

Several structured registration forms were distributed among PNs to gain insight into complete and acceptable delivery of steps of the home visitation programme. PNs received the [G]OLD-instrument on paper, which also included a form to register details of the post-discussion with the GP, and the care and treatment plan according to the official format as recommended by the Dutch College of General Practitioners. ${ }^{29}$ The care and treatment plan contained details per detected problem of the goal to be achieved, who will undertake action, and when evaluation will take place. Returned forms were checked for completeness and accuracy of reporting by a research assistant, thereby serving as a proxy for complete delivery of elements of the intervention protocol.

At the end of the 18-month follow-up period, PNs were asked to register on a structured form for each older person the number of follow-up contacts, the extent to which this person in general complied with follow-up actions or advice given (5-point 
scale: 'always', 'most of the time', 'sometimes', 'rarely', 'never', including the options 'I don't know' and 'not applicable, no action/help needed'), and the extent to which the older person in general benefited from follow-up actions, referral or advice given (4point scale: 'very much', 'somewhat', 'a little', 'not at all', including the reason for their judgement).

The two-day training programme for PNs was evaluated using a structured evaluation form to be filled-out at the end of each day. Besides their satisfaction with the training in general (on a scale ranging from 0 (worst score) to 10 (best score)), PNs were asked to what extent the training prepared them for the performance of the home visits and to what extent they felt confident that they could perform a home visit independently (both measured on 7-point scales ranging from 1 ('not at all') to 7 ('very much')).

At baseline, older people received the [G]OLD care diary to register details of their contacts with professionals offering care and/or well-being services during the 18month follow-up period. This information was intended as an indicator of compliance to follow-up actions besides the judgement of the PN. Unfortunately, only $7.1 \%$ of the older people ( $n=42$ out of 590 older people who had a home visit) filled-out the [G]OLD care diary and returned it at 18-months follow-up. Since this is not a representative sample for estimating compliance, the information was not used for process evaluation purposes.

\section{Continuous registration and notes}

The number of participants, non-participants, and drop-outs, including reasons for non-participation and drop-out were registered by members of the research team in the trial database. Furthermore, members of the research team made notes or narrative descriptions of the recruitment procedures and feedback meetings with all PNs together. These meetings were organised twice: after approximately six months and at the end of the intervention period. PNs were offered the possibility to exchange experiences and interim results of the individual semi-structured interviews were discussed (member check).

\section{Data analysis}

Descriptive statistics (e.g., means, frequencies, and percentages) were computed for quantitative process data using IBM SPSS Statistics for Windows, version 21.0. All individual semi-structured interviews with GPs, PNs, and older people from the intervention group were digitally recorded, after obtaining verbal consent from participants, and transcribed verbatim. The analysis of the interview transcripts was supported by the software package NVivo 7. Two members of the research team independently 
coded the data to enhance credibility of the findings. One member of the research team coded all transcripts, while a second coder served as a control and coded a random selection of one quarter of the transcripts to reduce workload. A general inductive approach was applied in which the coding process was largely guided by the process evaluation objectives. ${ }^{30}$ Systematic and rigorous reading and coding of the transcripts allowed major themes to emerge, which were compared for overlap. Consensus was reached after discussion and since the most important themes had already emerged, it was not considered necessary for the second coder to analyse the remaining transcripts. Other qualitative data (i.e., notes of the feedback sessions with PNs) were analysed using conventional content analysis ${ }^{31}$ to identify categories or new themes that did not yet emerge from the semi-structured interviews. Descriptions of procedures applied by the research team during the intervention period were summarised in a narrative report.

\section{RESULTS}

\section{Fidelity}

All steps of the home visitation programme were largely implemented as planned. Exceptions are changes in wording or omission of questions in the CGA by a few PNs, no or delayed post-discussion between the GP and PN, and no or inappropriately formulated care and treatment plans. Both PNs and GPs struggled most with how to arrange long-term monitoring of older people considering the limited number of hours PNs could dedicate to care for older people. Besides this, each PN developed his/her own routine in performing the different steps.

\section{Change in mindset}

The shift from reactively to proactively approaching older people in a structured and comprehensive way was evident for PNs, as well as for most GPs. One PN mentioned that GPs were not used to approaching older people proactively as they usually offer care and/or treatment upon request, whereas PNs are more familiar with delivering preventive care.

At the end of the intervention period, all general practices intended to continue with the home visitation programme, but the proactive versus a more reactive approach posed a dilemma for half of the practices. Proactively visiting all older people $(75+)$ allows for primary prevention of problems but is a huge time investment for general practices, while purposefully visiting older people who show signs of decline 
would be more feasible but at the expense of detecting problems which could have been prevented if addressed earlier.

\section{Interdisciplinary collaboration}

Due to the home visitation programme, PNs' network of professionals or disciplines involved in care for older people extended and they used their network to a greater extent. The number of referrals to secondary care was limited and the majority of contacts took place within primary care, for instance with home care organisations, physiotherapists, and occupational therapists. Several PNs indicated that they only had few contacts with other care professionals since not that many problems had been detected. Collaboration with other professionals was facilitated when they were located nearby, preferably in the same building.

In three general practices, multidisciplinary meetings took place on a structural base (e.g., once a month) in which older people were discussed from time to time as well. In other general practices, no structural meetings were organised yet with disciplines outside the general practice, mostly because no or only few complex problems were detected among older people. Three general practices had concrete plans to organise multidisciplinary meetings in the near future. Others were not convinced of their added value compared to existing meetings or contacts with other disciplines on an individual base.

\section{Decision-aids to support decision-making}

Half of the PNs made use of the service map offered by the project team with an overview of health and well-being facilities for older people and considered it useful. Others had not encountered any situations in which the service map could have been helpful.

Both PNs and GPs were positive about the [G]OLD-instrument, mostly because its extensiveness offered a comprehensive overview of the older person's health and well-being. However, the downside is that the instrument was time-consuming to administer. Consequently, several PNs and GPs sought for a balance in restricting the time investment without losing important content (e.g., application to a limited group of older people).

\section{Clinical information system}

Only in the region 'Parkstad', the digital system for registering the findings from the CGA and the care and treatment plan was finished at the start of the intervention period, although PNs could not yet register the results of monitoring and follow-up of older people over time. At first, PNs considered it time-consuming and double work. 
On the other hand, PNs liked the automatic link between the findings of the CGA and an inventory of detected problems and the care and treatment plan.

\section{Dose delivered (completeness)}

The dose delivered is illustrated in Figure 4.2. In total, 590 participants were visited at home by the PN for a CGA between July 2010 and September 2011. An underestimation of the actual number of post-discussions is likely, since not all PNs consistently filled-out the registration form. The percentage of formulated care and treatment plans per PN varied widely from $4.0 \%$ to $95.2 \%$. In case the format as handed over to PNs was not used, we did not count these as official care and treatment plans. Finally, we had no valid details per older person of their follow-up within the chain of care as PNs could not easily differentiate between follow-up contacts on behalf of the home visitation programme and other contacts with the general practice within the 18month period. Nonetheless, the follow-up process might have been suboptimal in several cases, as some PNs experienced time constraints and/or they did not have a concrete plan for monitoring and follow-up of older people over time.

\section{Dose received (exposure)}

According to the forms filled-out by PNs ( $n=384,65.1 \%)$, of the 229 older people who received follow-up actions or advice, $67.7 \%$ complied 'always' or 'most of the time', while $10.5 \%$ complied 'rarely' or 'never'. Six older people admitted during the interview that they did not comply with a specific advice given by the PN.

Half of the PNs noticed that they often came across older people who did not want any follow-up action(s) in the first place. Especially mental problems were difficult to deal with. Often older people agreed with actions to be undertaken when it was already too late. Some PNs struggled with how to deal with these older people and how to find a balance between respecting the older person's wishes and maintaining contact to try to achieve the desired actions over time. 


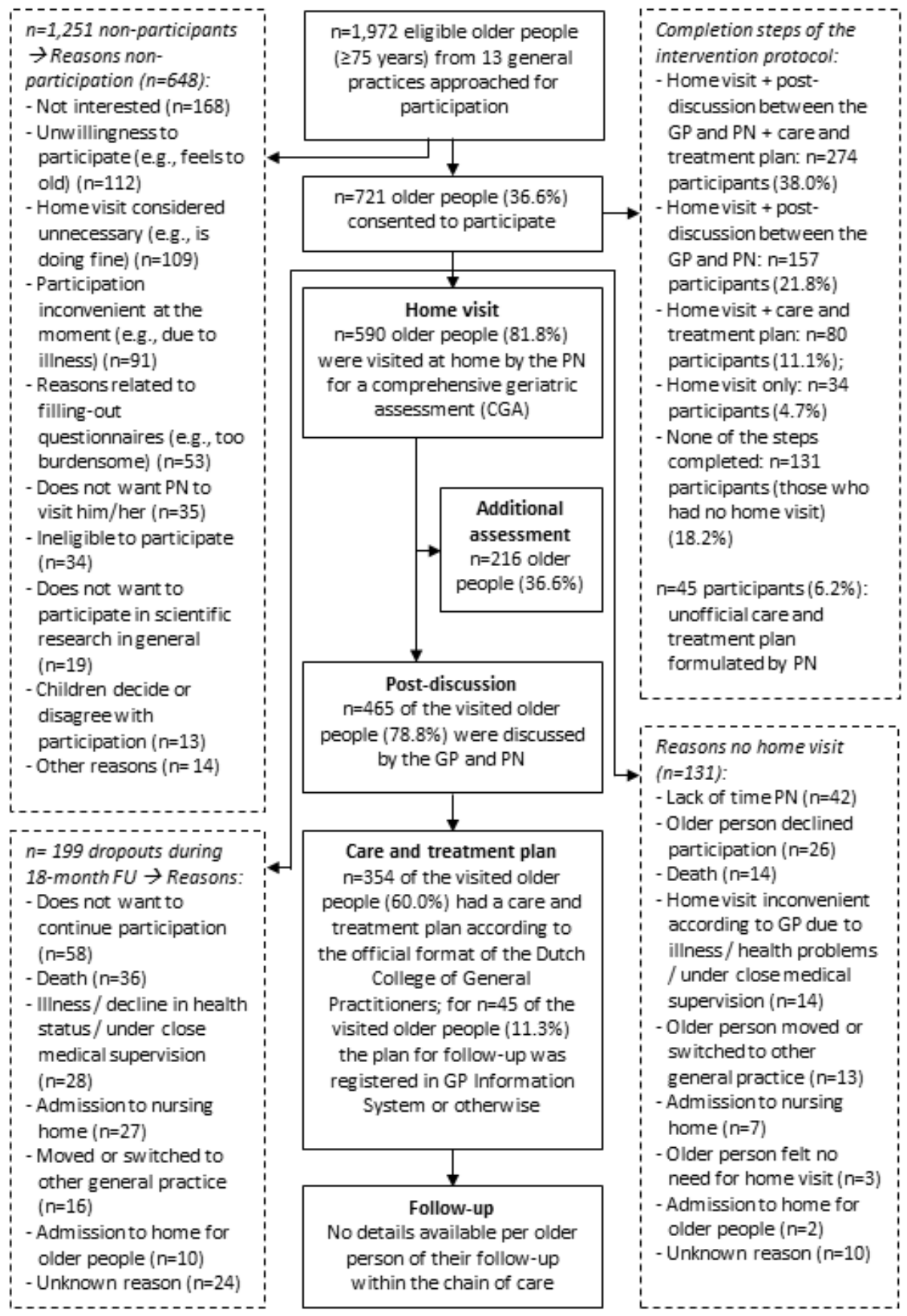

Figure 4.2 Dose delivered and reach of the [G]OLD home visitation programme 


\section{Dose received (satisfaction)}

\section{Practice nurses}

PNs liked the performance of the home visits most, due to the ability to get to know the older person and to offer help or advice. The home visit lowers the threshold for older people to contact the general practice should future problems arise. PNs were in favour of a home visit instead of a consultation in the general practice, as it offered a more objective picture of the older person's functioning. At the general practice level, the home visitation programme resulted in more attention for older people in general and closer collaboration between the GP and PN in organising care for older people. The majority of the PNs did not like the administrative work. Moreover, opinions of PNs were mixed regarding the usefulness of the care and treatment plan during referral and the follow-up process above the registration of the findings in the GP's Information System. In general, PNs evaluated the training as preparation for executing the home visitation programme rather positively $(M=7.64, S D=0.50$ for the first day vs. $M=6.64$. SD=0.92 for the second day).

\section{General Practitioners}

Half of the GPs liked most that they obtained a comprehensive and complete picture of older people's functioning and the social network surrounding older people. As a result, GPs considered the home visits useful, because it offered them additional information which might be valuable for future reference. At the general practice level, the home visitation programme had offered a starting point and useful tools for organising care for older people. Seven GPs mentioned that, against their expectations, no or only few previously unknown problems were detected. Furthermore, the older people that were out of the picture according to the GP and that would particularly benefit from the home visit often did not consent to participate.

\section{Older people}

Although the majority had no specific expectations about the home visit, three older people were hesitant about the purpose of the home visit at first. All older people were very satisfied with the home visit and afterwards, they had a good feeling about it or they emphasised that it had been interesting. They were very positive about the $\mathrm{PN}$ and felt that they could discuss everything with him/her. The home visit was neither too short nor too long and they felt that everything that they considered to be important was discussed. The questions asked as part of the CGA were not difficult to understand, impolite, awkward, or strange. Older people liked the ability to talk about different things, the unexpected attention from their general practice, and the fact that they now had a familiar face in the general practice. One person indicated that 
these visits tend to go towards too extensive meddling with other people's affairs, especially among older people who are doing relatively well and do not have a specific request for help.

\section{Benefits to older people}

According to the forms filled-out by PNs ( $n=394,66.8 \%$ ), for $29.9 \%$ of the older people follow-up actions, referral or advice had been 'very much' or 'somewhat' beneficial, while the remaining $70.1 \%$ of the older people benefited 'a little' or 'not at all'. Most PNs indicated that in the majority of the older people they did not achieve any major changes because no problems were detected or only problems that could easily be addressed or already had been taken care of. As recognised by a PN and GP and confirmed by one older person, sometimes older people did not optimally benefit from the home visit because they to some extent hold a façade of normalcy. Finally, one GP commented that the home visits are less useful for older people without a specific request for help as they often do not want to undertake action.

Six older people mentioned that the home visit had been useful for them, mostly because it lowered a threshold to discuss matters for which they do not easily contact the general practice themselves. Others believed that you are just old and there is nothing that can be done about that, and that certain problems (e.g., loneliness) cannot be solved.

\section{Reach}

Of the 1,972 eligible older people ( $\geq 75$ years) approached, $36.6 \%$ consented to participate (see Figure 4.2). Mean age of participants was 80.6 years (SD=4.26; range: 74.495.4) and $56.0 \%(n=972)$ were female. Participants were significantly younger compared to non-participants $(M=81.2, S D=4.39)(P=0.004)$ and men were 1.43 times more likely to participate than women. Participants who dropped out $(27.6 \%, n=199)$ during the 18-month follow-up period (see Figure 4.2), received usual care if necessary. Dropouts were significantly older $(M=81.1, S D=4.76)$ compared to those who continued participation ( $M=80.3, S D=3.95),(P=0.027)$ and women were equally likely to drop-out as men (OR=0.99). As Figure 4.2 shows, for only $38.0 \%$ of the 721 participants all steps of the [G]OLD-protocol up to the follow-up process were completed according to the registration forms filled-out by PNs.

Nearly all PNs and several GPs believed that they had missed the older people who would particularly benefit from the home visitation programme, since participants were the relatively healthy older people, those for whom care was already arranged quite well, or the ones who often visit the practice. They felt that due to the informed 
consent procedure of the trial, people who are not doing well or the more frail older people are suspicious about the consequences of participation.

\section{Recruitment}

All general practices in the regions 'Maastricht-Heuvelland' and 'Parkstad' were informed about the [G]OLD-project by means of a letter from the Primary Healthcare Organisation of their region, followed by information sessions and practice visits for those interested to participate. Non-responders were contacted by phone to inquire about their willingness to participate in the control group. Since insufficient general practices agreed to participate in the control group, jeopardising the continuation of the trial, the recruitment of control practices was extended to another region ('Midden-Limburg'). Eventually, 13 general practices were included in the intervention group ( 7 from the region 'Parkstad' and 6 from the region 'Maastricht-Heuvelland') and 11 general practices in the control group ( 2 from the region 'Parkstad' and 9 from the region 'Midden-Limburg').

Older people were approached for participation by means of an information letter and consent form. In the intervention group, those who did not return the signed consent form within two weeks were contacted by phone once to inquire whether they received the information letter. One postal reminder was send to older people who could not be contacted by phone. Due to the substantial time investment of calling older people, non-responders in the control group only received a postal reminder.

\section{Context}

\section{Barriers and facilitators for implementation}

Most of the barriers experienced by PNs during implementation were related to logistical difficulties in planning the different steps of the home visitation programme alongside other daily work. Especially the introduction of a new disease management programme for cardiovascular risk management (CVRM) by the primary care organisations during the intervention period posed challenges to PNs and GPs, causing several PNs to invest less time to the home visitation programme than planned. Finally, barriers for continuing the home visitation programme over time were the lack of an adequate reimbursement of the costs of care for older people and the overall time investment of the home visitation programme (total time investment from preparation of the home visit to formulating the care and treatment plan was on average 85 minutes per older person).

A facilitator for implementation according to several PNs was gaining routine in efficiently planning and executing the different steps of the home visitation pro- 
gramme. Moreover, some PNs expressed the need for regular meetings with other PNs to exchange experiences or the ability to consult an expert panel with practical questions. For GPs, having a PN in the general practice who is largely responsible for performing the home visitation programme and who gained experience in it, was a positive development conducive to successful implementation.

\section{Contamination control group}

None of the participating general practices in the control group had been involved in any form of proactive care for community-dwelling older people during the 18-month study period.

\section{Interactional workability}

All PNs were satisfied with how they worked together with the GP in deciding about follow-up actions for detected problems. Yet one PN and one GP noticed that sometimes there was incongruence between the two of them: the proactive approach required GPs to address different kinds of problems and/or needs that otherwise might not have received attention at this point in time. Mostly, older people agreed with the suggestions done by the PN for follow-up actions. However, both PNs and GPs observed that quite a few older people were not willing to undertake any follow-up actions or only when problems had progressed substantially (incongruence).

\section{Relational integration}

At the end of the training programme, PNs felt very confident that they could perform a home visit independently $(M=6.27, S D=0.91)$. In general, both PNs and GPs thought that the PN's knowledge, expertise or skills regarding care for older people were sufficient and, according to PNs, even increased during the intervention period. Those inexperienced with structural assessments would benefit from feedback, supplementary information, or examples on how to administer certain tests of the CGA and how to assign a score to the answers given by older people. A few PNs did not have that much expertise yet in deciding whether or not to undertake follow-up actions for detected problems and in formulating care and treatment plans correctly. One GP sensed a lack of affinity of the PN regarding care for older people. All older people believed the PN had sufficient knowledge about health, listened to them, took sufficient time for the home visit, and respected their needs and wishes.

\section{Skill-set workability}

Both PNs and GPs considered the division of work regarding the home visitation programme clear and acceptable. As GPs often cannot attribute as much time to older people as they would like, the expertise gained by PNs in care for older people was 
very much appreciated. One PN was a little disappointed that the GPs did not use her expertise more often in arranging follow-up actions for older people.

\section{Contextual integration}

Six PNs felt that the home visitation programme was well integrated within the healthcare services offered by the general practice. According to two GPs, the home visitation programme fits within the healthcare services offered by general practices, as older people are familiar with the general practice. Furthermore, it enabled GPs to be the central care provider and to collect information that is relevant to them.

According to PNs and GPs, sufficient time for care for older people was an essential resource for adequate performance of the home visitation programme. PNs' available time had to be carefully divided over various patient categories within the general practice. Five GPs thought that the time investment was disproportionate compared to the benefits in terms of detected problems. The other GPs thought the time investment was justifiable, as it yielded a lot more information about the older person.

Opinions of GPs diverged with respect to the importance of the reimbursement policy of health insurers for implementing care for (frail) older people. While some GPs considered it to be of minor importance, others believed its importance will grow over time due to competition between various disease management programmes for the available time of the PN. Finally, some GPs stated that continuation of the home visitation programme would largely depend on it.

\section{DISCUSSION}

This paper reports on a pre-planned, theory-based process evaluation into redesigning care delivery by general practices and implementation of a home visitation programme for potentially frail community-dwelling older people (aged $\geq 75$ years). Overall, the home visitation programme was delivered completely according to protocol to only $38.0 \%$ of the 721 study participants. This is considerably lower than the completion rate of $78 \%$ of a similar intervention. ${ }^{32}$ Several threats to complete delivery of the intervention have been identified.

First of all, lack of time emerged as a crucial factor in various elements of the process evaluation and prior research showed that it is an important barrier in providing structured care to older people in general practice. ${ }^{33,34}$ Administering the [G]OLDinstrument during the home visit, post-discussion between the PN and GP, administrative work (e.g., registering the findings from the CGA in the digital registration system), and monitoring older people were (initially) judged to be time-consuming activities or were influenced by time constraints of either the PN or GP. The time investment and 
corresponding available financial reimbursement were considered disproportionate compared to the benefits in terms of detected problems, thereby influencing the intention of general practices to continue using the home visitation programme. Furthermore, the time investment posed logistical challenges for some general practices in dividing the limited time of the PN over various patient categories. Interestingly, solutions to reduce the time investment were not sought in shortening the [G]OLDinstrument or adapting the intervention protocol in general, but in targeting the home visitation programme to a selected group of older people who benefit most from it.

Secondly, few new or complex health and/or well-being problems had been detected, which was the main cause of no post-discussions and no care and treatment plans for specific cases, and limited interdisciplinary cooperation. Both PNs and GPs believed that the relatively healthy older people were visited, suggesting inadequate reach. On the one hand, volunteer bias may have been introduced by the recruitment procedure on behalf of the parallel quasi-experimental trial. Alternatively, the population-based screening approach may have resulted in few (newly) detected problems among older people. While some opt for population-based screening of older people in general practice, ${ }^{6}$ other studies suggest that general practices benefit most from a more targeted screening approach. ${ }^{35,36}$

In agreement with Hasson and colleagues, ${ }^{37}$ we found that implementation fidelity was influenced by the care professionals' commitment to the home visitation programme, as well as their ability to execute the intervention protocol with the resources at hand. Both PNs and GPs were satisfied with the home visitation programme. It had resulted in more attention for older people, closer collaboration between the GP and $\mathrm{PN}$, and a comprehensive picture of older people's functioning and social network. This positive attitude made them willing to find solutions for barriers encountered during the implementation (e.g., monitoring older people via other disease management programmes). Such small changes to the intervention protocol were not considered threats to fidelity, but as necessary for translation of the home visitation programme into daily practice (i.e., work patterns of PNs). ${ }^{38}$ However, PNs struggled a lot with the follow-up process and thus, the extent of arranging follow-up actions and monitoring older people over time might have been limited, as also found in another recent study, ${ }^{25}$ threatening fidelity of implementation. Besides time constraints, other causes may be inadequate guidelines or decision-aids for PNs to arrange the monitoring process, some older people did not want to undertake follow-up actions, and a lack of adequate ICT-support to facilitate registration of the follow-up process.

With respect to redesigning care delivery for older people, shifting of care from the GP to the PN was evaluated positively and appeared to be conducive to the delivery of proactive care, as PNs in general are more familiar with a preventive approach. Nevertheless, some GPs were still more inclined towards offering reactive care. This 
implies that over time, GPs may relapse into their usual way of delivering care upon request (because of time constraints, lack of benefits in terms of detected problems, etc.), stimulated by the disproportionate cost-benefit ratio of the home visitation programme as mentioned earlier and the predominantly reactive healthcare system in the Netherlands.

All older people were satisfied about the home visit, regardless of whether problems had been detected, mostly because it offered them the ability to express their daily concerns. This 'attention' aspect of the home visit has been recognised before and is considered insufficient for eliciting substantial effects on patient outcomes. ${ }^{39}$

The development of a prospective process evaluation plan, underpinned by a theoretical framework, and using a mixed-method approach to data collection allowed for a high-quality and more thoroughly conducted process evaluation. Trustworthiness or credibility of the findings was enhanced using, among others, member checks, method triangulation (i.e., using multiple methods to collect data on a process evaluation question), and two independent coders during qualitative data-analysis. Nevertheless, the subjective experiences of PNs, GPs, and older people may have been subject to social-desirability bias or recall bias. Opinions of GPs might have been biased due to negotiations between insurers and care groups for a bundled payment system ${ }^{40}$ for complex care for older people. Honest responding by PNs may have been promoted by the independent role of the researcher and relationship of trust created due to repeated contacts during the intervention period. Further, despite using a varied sample of older people, we could not entirely circumvent that older people tend to provide less detailed descriptions of their experiences. ${ }^{41}$ Another limitation is that the structured registration forms were not always completely and accurately filled-out by PNs. This made them a less reliable source to assess for example implementation fidelity or dose delivered, and thereby also restricting method triangulation for certain process evaluation questions.

\section{CONCLUSIONS}

The current process evaluation offers useful insights for interpreting the results of the parallel quasi-experimental trial and for sustainability of the home visitation programme in general practices. ${ }^{42}$ The largest threats to positive outcomes at the patient level are the low dose delivered and inadequate reach. According to PNs, beneficial effects of the home visitation programme were 'little' or 'not at all' present in the majority of visited older people (70.1\%). Despite this, the home visitation programme was judged positively by both GPs and PNs and resulted in positive developments within the general practices. This suggests that the intervention does not have major 
shortcomings in itself, but the delivery offers room for improvement. Besides selecting the more frail community-dwelling older people with multiple and complex problems, alternative time-saving solutions for general practices may be sought in connecting general practices with initiatives in the neighbourhood or at the community level by developing welfare and care models. Regardless, the involvement of general practices is advocated ${ }^{4,5}$ and GPs also believe the home visitation programme fits within their range of care services. Finally, PNs would benefit from on-going training to update their knowledge and skills, thereby enhancing implementation fidelity, and to allow for exchanging experiences with other PNs. An imbalance between the time investment and available financial reimbursement in proportion to the number of meaningful problems detected among older people requires attention to enable continuation of the home visitation programme over time. The development of future complex interventions in the primary care setting should take into account that a pre-planned, theory-based process evaluation alongside the effect evaluation is inevitable to provide indepth insight into the actual performance of the intervention in the intended context. 


\section{REFERENCES}

1. Beswick AD, Rees K, Dieppe P, Ayis S, Gooberman-Hill R, Horwood J, et al. Complex interventions to improve physical function and maintain independent living in elderly people: a systematic review and meta-analysis. Lancet 2008;371:725-735.

2. Huss A, Stuck AE, Rubenstein LZ, Egger M, Clough-Gorr KM. Multidimensional preventive home visit programs for community-dwelling older adults: a systematic review and meta-analysis of randomized controlled trials. J Gerontol A Biol Sci Med Sci 2008;63A:298-307.

3. MacAdam M. Frameworks of integrated care for the elderly: a systematic review. Ontario: Canadian Policy Research Networks Inc., 2008.

4. Boeckxstaens P, De Graaf P. Primary care and care for older persons: position paper of the European Forum for Primary Care. Qual Prim Care 2011;19:369-389.

5. Lacas A, Rockwood K. Frailty in primary care: a review of its conceptualization and implications for practice. BMC Med 2012;10:4.

6. Fletcher AE, Price GM, Ng ESW, Stirling SL, Bulpitt CJ, Breeze E, et al. Population-based multidimensional assessment of older people in UK general practice: a cluster-randomised factorial trial. Lancet 2004;364:1667-1677.

7. Metzelthin SF, Van Rossum E, De Witte LP, Ambergen AW, Hobma SO, Siper W, et al. Effectiveness of interdisciplinary primary care approach to reduce disability in community dwelling frail older people: cluster randomised controlled trial. Br Med J 2013;347:f5264.

8. Vass M, Avlund K, Hendriksen C, Philipson L, Riis P. Preventive home visits to older people in Denmark. Why, how, by whom, and when? Z Gerontol Geriatr 2007;40:209-216.

9. Adams WL, Mcllvain HE, Lacy NL, Magsi H, Crabtree BF, Yenny SK, et al. Primary care for elderly people. Why do doctors find it so hard? Gerontologist 2002;42:835-842.

10. Stijnen MMN, Duimel-Peeters IGP, Jansen MWJ, Vrijhoef HJM. Early detection of health problems in potentially frail community-dwelling older people by general practices - project [G]OLD: design of a longitudinal, quasi-experimental study. BMC Geriatr 2013;13:7.

11. Bodenheimer T, Wagner EH, Grumbach K. Improving primary care for patients with chronic illness. J Am Med Assoc 2002;288:1775-1779.

12. Boult C, Karm L, Groves C. Improving chronic care: the "Guided Care" model. Perm J 2008;12:50-54.

13. Craig P, Dieppe P, Macintyre S, Michie S, Nazareth I, Petticrew M. Developing and evaluating complex interventions: the new Medical Research Council guidance. Br Med J 2008;337:a1655.

14. Oakley A, Strange V, Bonell C, Allen E, Stephenson J, RIPPLE Study Team. Process evaluation in randomised controlled trials of complex interventions. Br Med J 2006;332:413-416.

15. Bradley F, Wiles R, Kinmonth A-L, Mant D, Gantley M. Development and evaluation of complex interventions in health services research: case study of the Southampton heart integrated care project (SHIP). Br Med J 1999;318:711-715.

16. Stijnen MMN, Duimel-Peeters IGP, Vrijhoef HJM, Jansen MWJ. Process evaluation plan of a patientcentred home visitation program for potentially frail community-dwelling older people in general practice. Eur J Pers Cent Healthc 2014;2:179-189.

17. Harachi TW, Abbott RD, Catalano RF, Haggerty KP, Fleming CB. Opening the black box: using process evaluation measures to assess implementation and theory building. Am J Community Psychol 1999;27:711-731.

18. Reelick MF, Faes MC, Esselink RAJ, Kessels RPC, Olde Rikkert MGM. How to perform a preplanned process evaluation for complex interventions in geriatric medicine: exemplified with the process evaluation of a complex falls-prevention program for community-dwelling frail older fallers. J Am Med Dir Assoc 2011;12:331-336. 
19. Saunders RP, Evans MH, Joshi P. Developing a process-evaluation plan for assessing health promotion program implementation: a how-to guide. Health Promot Pract 2005;6:134-147.

20. Baranowski T, Stables G. Process evaluations of the 5-a-day projects. Health Educ Behav 2000;27:157166.

21. Linnan L, Steckler A. Process evaluation for public health interventions and research. An overview. In: Steckler A, Linnan L, editors. Process evaluation for public health interventions and research. San Francisco: Jossey-Bass; 2002. p. 1-24.

22. May CR. A rational model for assessing and evaluating complex interventions in health care. BMC Health Serv Res 2006;6:86.

23. Bleijenberg N, Ten Dam VH, Drubbel I, Numans ME, De Wit NJ, Schuurmans MJ. Development of a proactive care program (U-CARE) to preserve physical functioning of frail older people in primary care. J Nurs Scholarsh 2013;45:230-237.

24. Melis RJF, Van Eijken MIJ, Boon ME, Olde Rikkert MGM, Van Achterberg T. Process evaluation of a trial evaluating a multidisciplinary nurse-led home visiting programme for vulnerable older people. Disabil Rehabil 2010;32:937-946.

25. Metzelthin SF, Daniëls R, Van Rossum E, Cox K, Habets H, De Witte LP, et al. A nurse-led interdisciplinary primary care approach to prevent disability among community-dwelling frail older people: a large-scale process evaluation. Int J Nurs Stud 2013;50:1184-1196.

26. Stijnen MMN, Jansen MWJ, Vrijhoef HJM, Duimel-Peeters IGP. Development of a home visitation programme for the early detection of health problems in potentially frail community-dwelling older people by general practices. Eur J Ageing 2013;10:49-60.

27. Edmonds WA, Kennedy TD. An applied reference guide to research designs. Quantitative, qualitative and mixed methods. Thousand Oaks, CA: Sage; 2013.

28. Creswell JW, Fetters MD, Ivankova NV. Designing a mixed methods study in primary care. Ann Fam Med 2004;2:7-12.

29. Dutch College of General Practitioners. NHG-Praktijkwijzer Ouderenzorg [Dutch College of General Practioners-Manual care for older people in general practice]. Utrecht: NHG, 2010.

30. Thomas DR. A general inductive approach for analyzing qualitative evaluation data. Am J Eval 2006;27:237-246.

31. Hsieh H, Shannon SE. Three approaches to qualitative content analysis. Qual Health Res 2005;15:12771288.

32. Bouman A, Van Rossum E, Habets H, Kempen GIJM, Knipschild P. Home visiting program for older people with health problems: process evaluation. J Adv Nurs 2007;58:425-435.

33. Bleijenberg N, Hester-Ten Dam V, Steunenberg B, Drubbel I, Numans ME, De Wit NJ, et al. Exploring the expectations, needs and experiences of general practitioners and nurses towards a proactive and structured care programme for frail older patients: a mixed-methods study. J Adv Nurs 2013;69:22622273.

34. Evans C, Drennan V, Roberts J. Practice nurses and older people: a case management approach to care. J Adv Nurs 2005;51:343-352.

35. De Lepeleire J, Iliffe S, Mann E, Degryse J. Frailty: An emerging concept for general practice. Br J Gen Pract 2009;59:e177-e182.

36. Iliffe $\mathrm{S}$, Orrell $\mathrm{M}$. Identifying unmet health needs in older people: comprehensive screening is not the answer. Br J Gen Pract 2006;56:404-406.

37. Hasson $\mathrm{H}$, Blomberg $\mathrm{S}$, Dunér A. Fidelity and moderating factors in complex interventions: a case study of a continuum of care program for frail elderly people in health and social care. Implement Sci 2012;7:23.

38. Glasgow RE, Emmons KM. How can we increase translation of research into practice? Types of evidence needed. Annu Rev Public Health 2007;28:413-433. 
39. Van Haastregt JCM, Van Rossum E, Diederiks JPM, De Witte LP, Voorhoeve PM, Crebolder HFJM. Process-evaluation of a home visit programme to prevent falls and mobility impairments among elderly people at risk. Patient Educ Couns 2002;47:301-309.

40. Struijs JN, Baan CA. Integrating care through bundled payments - Lessons from the Netherlands. N Engl J Med 2011;364:990-991.

41. Kirkevold $M$, Bergland $A$. The quality of qualitative data: issues to consider when interviewing participants who have difficulties providing detailed accounts of their experiences. Int J Qual Stud Health Well-being 2007;2:68-75.

42. Sibthorpe BM, Glasgow NJ, Wells RW. Emergent themes in the sustainability of primary health care innovation. Med J Aust 2005;183:S77-S80. 



\section{Formulation of and adherence to a care plan for potentially frail community-dwelling older people by practice nurses in the Netherlands}

Published as:

Stijnen MMN, Vrijhoef HJM, Duimel-Peeters IGP, Jansen MWJ. Formulation of and adherence to a care plan for potentially frail community-dwelling older people by practice nurses in the Netherlands. J Nurs Educ Pract, 2014;4:34-43. 


\section{ABSTRACT}

\section{Background}

In the Netherlands, practice nurses within general practices increasingly deliver proactive care to older people. As part of a home visitation programme, they assess older people's health status during a home visit, followed by formulating a care plan and monitoring follow-up care. Insight into the latter two steps is limited, whereas previous research shows signs of inadequate follow-up, threatening the achievement of optimal patient outcomes. The objectives of the current study are to investigate practice nurses' actions and views regarding (1) the formulation of a care plan for potentially frail community-dwelling older people following a comprehensive geriatric assessment during a home visit and (2) their adherence to the care plan and monitoring during follow-up of older people in whom health and/or well-being problems have been detected.

\section{Methods}

A cross-sectional mixed-model study was conducted. Results of the comprehensive geriatric assessment were compared to the care plans to reveal the percentage of problems included. Semi-structured interviews were performed with twelve practice nurses who visited older people at home. Per practice nurse, care plans of ten older people (total $n=120$ ) were purposefully selected and discussed to identify reasons for not registering problems in the care plan and to determine whether and how problems were monitored.

\section{Results}

Of the problems identified through comprehensive geriatric assessment, $27.8 \%$ $(n=275)$ were included in a care plan of which $62.9 \%(n=173)$ were addressed using follow-up actions. Patient-related and problem-specific factors influenced the formulation of care plans. Insufficient time challenged practice nurses in monitoring older people over time.

\section{Conclusions}

The formulation of care plans and monitoring older people over time offers room for improvement. Once problems are included into the plans, practice nurses mostly adhere to the care plans. 


\section{INTRODUCTION}

As the ageing of the population continues, the accompanying rise in frail older people poses a high burden on the healthcare system. Frail older people are at increased risk of adverse outcomes such as falls, disability, and hospitalisation. ${ }^{1}$ In targeting frail older people, early detection of problems and needs seems beneficial as it offers primary care professionals an opportunity to discuss goals of care and informs proactive decisions in planning care, treatment, and well-being services. ${ }^{2}$ As a result, within general practices in the Netherlands, shifting of care from general practitioners (GPs) towards practice nurses (PNs) ${ }^{3}$ has extended from chronic disease management to proactive care for potentially frail older people.

One way to organise nurse-based primary care for older people is by applying certain generic, evidence-based principles for the effective management of chronic diseases in primary care that are associated with improved health outcomes, such as targeted assessment, a personalised care plan for goal achievement, and active followup. ${ }^{4}$ These elements are incorporated in a nurse-delivered preventive home visitation programme implemented in general practices in the south of the Netherlands. The [G]OLD home visitation programme ('Getting OLD the healthy way') is aimed at the early identification of health and/or well-being problems followed by, if required, individualised care and follow-up with the ultimate goal to maintain independent living among potentially frail community-dwelling older people ( $\geq 75$ years). ${ }^{5}$ The PN performs an assessment of the person's health problems and needs using the [G]OLD comprehensive geriatric assessment (CGA) during a home visit. Based on the results and discussion with the GP, the PN formulates a personalised care plan with written agreements between the GP/PN and the older person concerning follow-up actions to be undertaken. Hereafter, the PN coordinates referral to care and/or well-being facilities, monitors progress, and evaluates whether changes according to the agreements written down in the care plan have occurred or new problems have arisen since the last visit.

Home visitation programmes that include extended follow-up and evaluation of the implementation of recommendations for problems identified through CGA are more likely to be effective, ${ }^{6,7}$ although many studies do not provide details on these aspects. ${ }^{8}$ The care providers' perspective of planning these care and treatment recommendations and organising follow-up is largely neglected. Yet, less-than-optimal patient outcomes can not only be attributed to a lack of adherence by older people to care plans but also to inadequate follow-up by the care provider. ${ }^{9} \mathrm{~A}$ recent process evaluation of a home visitation programme found that the extent of evaluation of care and treatment recommendations and follow-up over time by care providers was limited. ${ }^{10}$ Thus, the aim of this study was to investigate PNs' actions and views regarding 
(1) the formulation of a care plan for potentially frail community-dwelling older people following a CGA during a home visit and (2) their adherence to the care plan and monitoring during follow-up of older people in whom health and/or well-being problems have been detected.

\section{METHODS}

\section{Study design}

A cross-sectional study was conducted. To yield an enriched understanding of the topic of interest, both quantitative and qualitative methods were used. This resulted in a mixed-model design, or more specifically a convergent-parallel approach and data transformation design. ${ }^{11}$ Quantitative and qualitative data were given equal priority, they were gathered concurrently, and mixing took place at the stages of research question formulation, data analysis, and interpretation (see Figure 5.1).

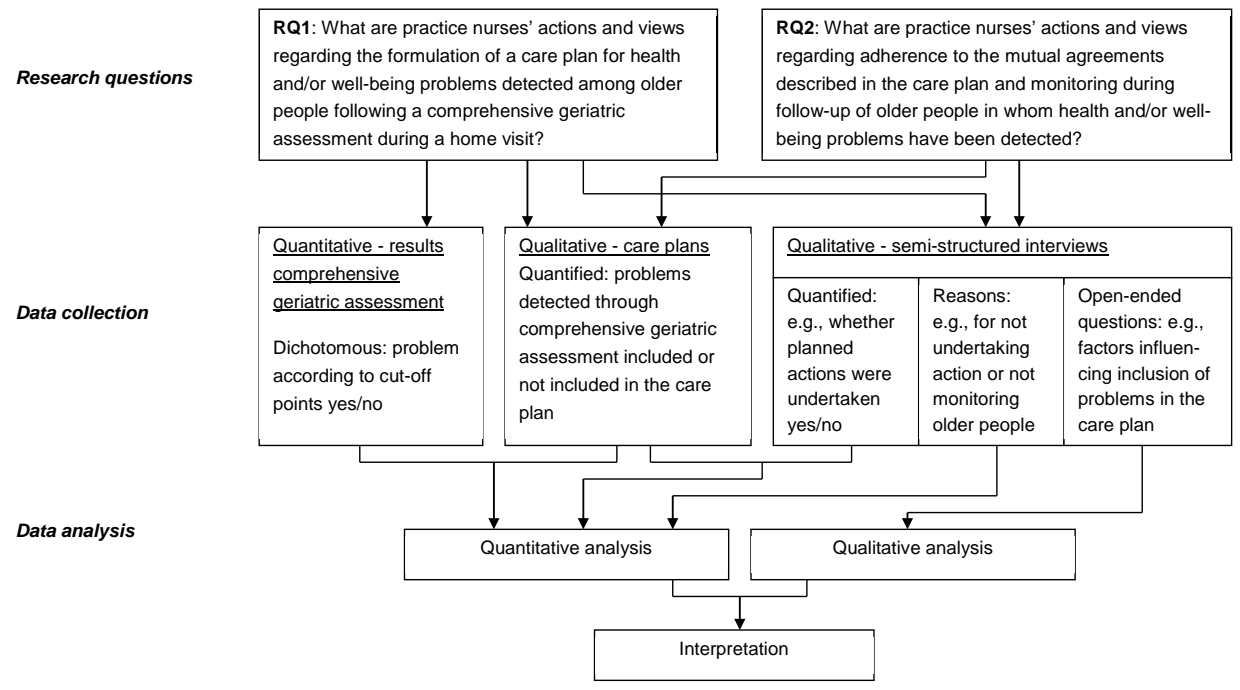

Figure 5.1 Visualisation of the mixed-model research design used in this study

\section{Setting and participants}

Thirteen PNs performed home visits on behalf of a quasi-experimental controlled trial. At that time, PNs were not informed about an interview to evaluate the formulation of and adherence to the care plans. Approximately one year after finalising the home 
visits for the trial, PNs were approached to participate in a semi-structured interview for this study. Twelve PNs (92.3\%) from 12 general practices in the south of the Netherlands verbally consented to participate; one PN withdrew from participation due to time constraints and work commitments.

Between July 2010 and September 2011, 12 PNs visited 565 older people, with an average of 47.1 home visits per PN (SD=19.6; range: 13-78). Per PN, we purposefully selected ten cases they visited before in which the most health and/or well-being problems had been detected to have sufficient input for the semi-structured interviews (total $n=120$ cases). In this way, we intended to obtain a comprehensive understanding of the decision-making process, while keeping the time investment for PNs to discuss the cases to a minimum (i.e., maximum interview duration of one hour).

\section{Data collection}

\section{Comprehensive geriatric assessment}

Our CGA comprises an assessment by the PN of older people's physical functioning (e.g., blood pressure, mobility), psychological and mental functioning (e.g., cognition, depression), social functioning (e.g., loneliness), lifestyle, and medication use. During the home visit, the PN registered answers to the items included in the [G]OLDinstrument manually on a structured registration form. For each test or question in the CGA, evidence-based or practice-based cut-off points were provided to help decide whether a problem required further attention (dichotomous).

\section{Care plan}

The care plan used by PNs originated from the format as recommended by the Dutch College of General Practitioners. ${ }^{12}$ For each problem, PNs were requested to indicate the goal to be reached; who will undertake what action; and at what date evaluation will take place. A case-manager was assigned who is responsible for care coordination, monitoring, and evaluation. PNs received brief training how to use the care plan, as this aspect was covered extensively during their training as PN. A researcher (MS) and project assistant (MM) compared the results of the CGA to the formulated care plan for each case in order to determine whether problems had been included (dichotomous).

\section{Semi-structured interviews}

The semi-structured interviews $(n=12)$ were digitally recorded and transcribed verbatim. Interviews took place between May and July 2012 with each PN individually at the general practice. Each interview began by asking open-ended questions regarding which factors in general influenced the decision to register detected problems in the 
care plan. Subsequently, each selected case was discussed with the PN. Questions included whether a problem in the care plan was monitored and evaluated, and reasons per detected problem for not including it in the care plan or not having monitored it during follow-up. Interviews lasted on average 50.2 minutes (SD=10.6; range: 39.268.5).

\section{Data analysis}

Qualitative data were analysed using NVivo version 7, in which transcripts were transferred anonymously. A researcher (MS) and project assistant (MM) coded the transcripts independently and parallel. Two sets of categories emerged that were combined after comparing for overlap. Any inconsistencies were resolved through discussion with other members of the research team (HV, ID, and MJ). A general inductive approach $^{13}$ was applied for data analysis to derive findings in the context of the research questions guiding this study. Systematic and rigorous reading and coding of the transcripts allowed major themes to emerge.

Parts of the qualitative data (i.e., how the care plan for each case was monitored and evaluated) were converted into numerical codes that were then analysed quantitatively using SPSS version 17.0. Furthermore, the results of the CGA in terms of detected problems and needs (dichotomous) per older person were compared with the accompanying care plan (problem included or not included; dichotomous). Descriptive statistics (e.g., frequencies) were computed for quantitative data.

\section{RESULTS}

\section{Characteristics practice nurses}

Participating PNs had a mean age of 40.9 years ( $S D=10.2$; range: $28.6-59.1$ ) and were predominantly female (91.7\%). PNs had an educational background in nursing (33.3\%), nursing complemented with training as PN (33.3\%), physician assistance complemented with training as PN (25.0\%), and psychology complemented with training as PN (8.4\%). Their mean working experience as $\mathrm{PN}$ was 4.5 years $(\mathrm{SD}=1.8$; range: $2.3-8.2$ ).

\section{Characteristics older people}

The age of selected cases at the time of the home visit ranged from 74.8-95.5 years $(\mathrm{Mdn}=80.6)$ and $54.2 \%$ was female. They did not statistically differ from the remaining 445 older people visited by the $P N s$ with respect to age, $U=24317.50, P=0.15, r=-0.06$ 
and gender, $\chi^{2}=0.70, d f=1, P=0.40$. As intended, significantly more problems were detected among the selected cases ( $M d n=8.0$; range: 6-10) compared to those not included in this study (Mdn=5.0; range: $3-7$ ), $U=11302.00, P<0.001, r=-0.41$.

\section{Formulation of care plan}

\section{Actions in general}

For nine cases (7.5\%), PNs considered a care plan to be unnecessary ( $M d n=7.0$ problems; range: 5-8.5) (e.g., detection of known and already addressed problems). For 94 cases $(78.3 \%)$, they formulated a care plan according to the format of the Dutch College of General Practitioners ( $M d n=8.0$ problems; range: 6-10). Two PNs documented follow-up actions in the GP Information System for 17 cases (14.2\%) (Mdn=9.0 problems; range: 7-12). Since these did not contain all necessary details, we did not count them as care plans. The average percentage of detected problems included in care plans (completeness) varied considerably between PNs (range: 10.8\%-52.9\%).

\section{Actions per problem}

In total, 989 problems were detected among the cases of which $27.8 \%$ ( $n=275)$ were included in a care plan. Problems/needs most often included were need for help in (I)ADL (55.6\%), financial problems (50.0\%), and depressive complaints (45.5\%). Least often included were smoking $(0 \%), \mathrm{BMI}<20$ or $>27(1.8 \%)$, and health deterioration in past 12 months (3.5\%) (Table 5.1). The reason mentioned most by PNs for not including specific problems in the care plan was that it was an existing problem and/or action had already been undertaken (13.5\%) (Table 5.2).

\section{Practice nurses' views}

Besides the cut-off points in the [G]OLD-instrument, other factors influenced the decision to include a problem in the care plan, such as the seriousness of the problem:

[...] to what extent does it often return during the conversation, a particular subject. How emotionally charged is it. And well, then I think like if something is a one-time event then I do not immediately consider it a problem. (PN8)

In addition, the older person's wishes were considered:

If the client clearly says I don't want that and I have the feeling like well, I dare to make the decision to not do anything with it, then I also leave it like that. (PN10) 
Another factor involved to what extent undertaking action was still attainable at the older person's age:

And then the question is do you still want to undertake such things if people have a certain age, so to say. (PN6)

Finally, the GP's opinion and available knowledge concerning the older person's health status played a role in deciding whether to include a problem in the care plan:

And, we've had this as well, that just ... that someone did not know one word that well or did the clock slightly wrong and that from the experience the GP had with this person, we said like at this moment we don't do anything with it. (PN2)

Lifestyle-related behaviours such as smoking and physical exercise were often not included in the plan, because PNs assumed that dietary and lifestyle patterns were addressed in other disease management programmes, action often only consisted of providing advice, or older people were reluctant to change their behaviour.

\section{Adherence to care plan and monitoring during follow-up}

\section{Actions in general}

Of the 120 cases, $71.7 \%(n=86)$ were monitored and this mainly took place via other disease management programmes $(n=35,29.2 \%)$ (Table 5.3). The PN who performed the home visit was most often responsible for monitoring and evaluation.

\section{Actions per problem}

In total, 173 problems were addressed by means of follow-up actions, which is $62.9 \%$ of all problems registered in a care plan $(n=275)$ and $17.5 \%$ of all identified problems through the CGA ( $n=989$ ). The problems addressed most often were health deterioration in past 12 months, discrepancy between registered and actual medication use, $\mathrm{BMI}<20$ or $>27$, faecal incontinence, and loneliness (all 100\%). Decreased appetite, smoking, excessive alcohol use, and absence of social network were dealt with the least (all 0\%) (Table 5.1). Most often, actions according to the care plan were not undertaken because the older person did not wish so (36.3\%) (Table 5.4). 
Table 5.1 Course of action practice nurse per detected problem in the CGA among selected cases $(n=120)$

\begin{tabular}{|c|c|c|c|c|c|c|c|}
\hline \multirow[t]{2}{*}{$\begin{array}{l}\text { (Early signs of) problems according to cut-off } \\
\text { points comprehensive geriatric assessment }\end{array}$} & \multirow[b]{2}{*}{$\mathrm{N}$} & \multicolumn{2}{|c|}{ Prevalence } & \multicolumn{2}{|c|}{$\begin{array}{l}\text { Included } \\
\text { in plan }\end{array}$} & \multicolumn{2}{|c|}{$\begin{array}{l}\text { Action } \\
\text { undertaken }\end{array}$} \\
\hline & & $\mathrm{n}$ & $\%$ & $\mathrm{n}$ & $\%^{*}$ & $\mathrm{n}$ & $\%^{+}$ \\
\hline \multicolumn{8}{|l|}{ General health problems } \\
\hline Complaints past 1-2 months & 120 & 74 & 61.7 & 22 & 29.7 & 14 & 63.6 \\
\hline Health deterioration in past 12 months & 120 & 57 & 47.5 & 2 & 3.5 & 2 & 100 \\
\hline \multicolumn{8}{|l|}{ Problems medication use } \\
\hline Polypharmacy (daily intake of $\geq 5$ medications) & 117 & 67 & 57.3 & 29 & 43.3 & 17 & 58.6 \\
\hline Discrepancy registered and actual medication use & 114 & 17 & 14.9 & 4 & 23.5 & 4 & 100 \\
\hline \multicolumn{8}{|l|}{ Physical problems and lifestyle } \\
\hline Systolic blood pressure $>140 \mathrm{mmHg}$ & 119 & 64 & 53.8 & 29 & 45.3 & 25 & 86.2 \\
\hline Fall incidents in past 6 months & 120 & 62 & 51.7 & 21 & 33.9 & 12 & 57.1 \\
\hline Body Mass Index (BMI) $<20$ or $>27 \mathrm{~kg} / \mathrm{m}^{2}$ & 109 & 56 & 51.4 & 1 & 1.8 & 1 & 100 \\
\hline Insufficient physical activity & 119 & 59 & 49.6 & 6 & 10.2 & 4 & 66.7 \\
\hline Impaired hearing & 110 & 51 & 46.4 & 19 & 37.3 & 10 & 52.6 \\
\hline Urine incontinence & 120 & 53 & 44.2 & 10 & 18.9 & 6 & 60.0 \\
\hline Decreased mobility & 119 & 52 & 43.7 & 17 & 32.7 & 12 & 70.6 \\
\hline Impaired sight & 119 & 51 & 42.9 & 13 & 25.5 & 6 & 46.2 \\
\hline Decreased appetite & 119 & 20 & 16.8 & 1 & 5.0 & 0 & 0 \\
\hline Need for help (instrumental) activities of daily living & 116 & 18 & 15.5 & 10 & 55.6 & 8 & 80.0 \\
\hline Smoking & 119 & 14 & 11.8 & 0 & 0 & 0 & 0 \\
\hline Excessive alcohol use & 76 & 6 & 7.9 & 1 & 16.7 & 0 & 0 \\
\hline Undesired weight loss & 112 & 7 & 6.3 & 2 & 28.6 & 1 & 50.0 \\
\hline Faecal incontinence & 120 & 6 & 5.0 & 1 & 16.7 & 1 & 100 \\
\hline \multicolumn{8}{|l|}{ Psychological and mental problems } \\
\hline Memory problems & 118 & 96 & 81.4 & 39 & 40.6 & 20 & 51.3 \\
\hline Personality disorder & 119 & 30 & 25.2 & 6 & 20.0 & 1 & 16.7 \\
\hline Concerned about falling & 120 & 28 & 23.3 & 9 & 32.1 & 8 & 88.9 \\
\hline Depressive complaints & 120 & 22 & 18.3 & 10 & 45.5 & 5 & 50.0 \\
\hline Anxiety & 120 & 12 & 10.0 & 4 & 33.3 & 2 & 50.0 \\
\hline \multicolumn{8}{|l|}{ Social problems } \\
\hline Burdened by informal care giving & 19 & 8 & 42.1 & 3 & 37.5 & 2 & 66.7 \\
\hline Concerns about informal caregiver & 54 & 14 & 25.9 & 3 & 21.4 & 2 & 66.7 \\
\hline Loneliness & 119 & 25 & 21.0 & 7 & 28.0 & 7 & 100 \\
\hline Absence of social network & 115 & 8 & 7.0 & 1 & 12.5 & 0 & 0 \\
\hline Unsatisfied with daily routines & 119 & 8 & 6.7 & 3 & 37.5 & 2 & 66.7 \\
\hline Financial problems & 119 & 4 & 3.4 & 2 & 50.0 & 1 & 50.0 \\
\hline
\end{tabular}

*Percentages are based on the numbers presented in the column 'Prevalence'.

'Percentages are based on the numbers presented in the column 'Included in plan'. 


\section{CHAPTER 5}

Table 5.2 Reasons practice nurses for not including a specific problem in the care plan ( $\mathrm{n}=714$ problems)

\begin{tabular}{lll}
\hline & $\mathrm{n}$ & $\%$ \\
\hline Existing problem and/or action has already been undertaken & 96 & 13.5 \\
Older person does not wish any action & 53 & 7.4 \\
Score just below cut-off point, no reason yet for further action & 36 & 5.1 \\
No change possible anymore & 24 & 3.4 \\
Advice given, no further action needed & 21 & 2.9 \\
Not considered a problem (anymore) by the older person & 19 & 2.7 \\
Additional examination revealed it was not a problem after all & 18 & 2.5 \\
Explicable, no further action needed according to the GP & 11 & 1.5 \\
Older person undertakes action himself/herself & 3 & 0.4 \\
Watchful waiting policy & 3 & 0.4 \\
No appropriate services available to address problem & 1 & 0.1 \\
Other: PN did not formulate a care plan & 217 & 30.4 \\
Other: Reason unknown & 212 & 29.7 \\
\hline
\end{tabular}

Table 5.3 Arrangement long-term monitoring of older people by practice nurses after the initial home visit $(n=120)$

\begin{tabular}{lll}
\hline & $\mathrm{n}$ & $\%$ \\
\hline Via contacts on behalf of disease management programmes & 35 & 29.2 \\
Home visits at fixed time points & 21 & 17.5 \\
PN assigned monitoring to another caregiver (formal or informal) & 19 & 15.8 \\
Mutual agreement that older person contacts PN when needed & 5 & 4.2 \\
Telephone calls at fixed time points $_{\text {Consultation in the general practice at fixed time points }}^{*}$ & 3 & 2.5 \\
No monitoring $^{*}$ & 3 & 2.5 \\
\hline
\end{tabular}

* No monitoring because no further actions were needed $(n=16,47.1 \%)$, older person is lost out of sight $(n=10,29.4 \%)$, or an event in the older person's life causing discontinuation of monitoring (e.g., illness, death, left the practice, admission to nursing home) $(n=8,23.5 \%)$. 
Table 5.4 Reasons practice nurses for not undertaking action for problems in the care plan ( $\mathrm{n}=102$ problems)

\begin{tabular}{lll}
\hline & $\mathrm{n}$ & $\%$ \\
\hline Older person does not wish any action & 37 & 36.3 \\
Existing problem and/or action has already been undertaken & 20 & 19.6 \\
Due to older person's medical circumstances (e.g., ill, admitted to nursing home & 11 & 10.8 \\
or home for older people, deceased) & & 8.8 \\
Additional examination revealed it was not a problem after all & 9 & 7.8 \\
No change possible anymore & 8 & 3.9 \\
Forgotten to monitor & 4 & 3.9 \\
Not considered a problem (anymore) by the older person & 4 & 2.9 \\
Not necessary anymore, older person's status improved & 3 & 2.0 \\
Watchful waiting policy & 2 & 2.0 \\
Explicable, no further action needed according to the GP & 2 & 2.0 \\
PN cannot recall reason & 2 & \\
\hline
\end{tabular}

Practice nurses' views

In general, PNs deemed monitoring of older people over time important to get an overview of the success rate of the follow-up actions undertaken. However, sometimes there was no need for monitoring (e.g., when only advice was given) or monitoring was considered unnecessary once the goal according to the care plan was reached. No time for monitoring and evaluation was a prominent issue raised and most PNs struggled how to shape the process of monitoring given the limited hours they could dedicate to care for older people:

So now, I only spend four hours a week to care for older people. [...] And then I notice that sometimes that goes by the board you know, I am honest in that. Then I think, actually I would want to call these people now to see how they are doing, what to do next. (PN2)

PNs often encountered older people who refused follow-up actions (care avoiders) and who only agreed with actions to be undertaken when their health status decreased significantly. These people challenged PNs in how to prevent losing them out of sight:

[...] we decided like make sure to keep in touch, gaining a bit of confidence and then little by little you try to start some care in this way. (PN1) 


\section{DISCUSSION}

Besides case management for people with chronic diseases, practice nurses within general practices in the Netherlands are increasingly held responsible for organising care for older people. The present study examined into more detail how PNs formulate and adhere to a care plan in delivering proactive care to community-dwelling older people.

Although nurse-led chronic disease management has proven to be feasible, acceptable, and sustainable in general practice, ${ }^{14}$ proactive care for older people poses additional challenges to PNs. Concerning the formulation of care plans, only $27.8 \%$ of the problems identified through CGA were included in a care plan, which is in agreement with our previous work. ${ }^{15}$ It implies, as confirmed by the findings, that the formulation of care plans by PNs is not only influenced by the cut-off points of the CGA, but also by a complex interplay of patient-related and problem-specific factors. In decisionmaking, PNs may outweigh the importance of various factors based on their knowledge of the older person ${ }^{16}$ and other key aspects known to influence nursing decision-making, such as expertise and intuition. ${ }^{17}$ Moreover, the post-discussion of each CGA with the GP could have influenced the decision to prioritise problems typically identified under a more medical model (e.g., hypertension) at the expense of more client-centred problems. Further research is needed to support this assertion.

Also consistent with our previous work, ${ }^{15}$ we found that the main reason for not including the remaining $72.2 \%$ of the problems in the care plan was that the problem was already known and/or action had already been undertaken. Hence, it might be argued that the ability of the [G]OLD-instrument to identify new or unmet needs that require follow-up actions is limited. Alternatively, older people who consented to participate in the trial and were visited by PNs may have been those who frequently visit their GP or they are healthier than their non-participating counterparts, suggesting volunteer bias. $^{18}$

Although few problems were registered in a care plan, $62.9 \%$ of these problems were dealt with according to PNs, suggesting a high adherence to the care plan approximately one year after it was formulated. This percentage is higher than reported by Piccoliori et al. ${ }^{19}$ (47\% of the problems were dealt with after one year), and might be explained by the involvement of the $\mathrm{PN}$ as case-manager who can attribute more time to care planning and coordination than GPs. However, an often-mentioned reason by PNs for not monitoring during follow-up was a lack of time. At the time of our study, general practices were not separately paid for care coordination and other services performed outside the initial home visit. As a result, PNs may not have been able to devote as much time to care coordination as they wanted. The importance of time in PNs' adherence to case management elements has been identified before. ${ }^{20,21}$ 
The findings imply that training for PNs targeted at acquiring additional knowledge and skills in formulating care plans may improve its adequate formulation and equalise undesired variation between PNs in the completeness of formulated care plans. In addition, the recommended format for the care plan may require adjustments to fit PNs' needs. Furthermore, PNs should be allowed sufficient time to monitor and evaluate care provided to older people. With the increased workload imposed on PNs, these case management steps are most likely to be discarded first. To deal with this issue, a transition from the current fee-for-service payments to a bundled payment system (i.e., health insurers pay a single fee to a multidisciplinary care group to cover different elements of care, for example for patients with chronic diseases) ${ }^{22}$ may be considered.

A limitation of the current study is that PNs and the researcher who conducted the interviews knew one another from earlier contacts on behalf of the trial. Although this may have influenced the answers given by PNs, it can also have created a relationship of trust and thereby fostered sincerity in PNs' responses. The researcher's independent role and asking questions in a non-offending way concerning a possibly sensitive subject as adherence to case management steps may also have been conducive to honest responding by PNs. The questions asked necessitated PN to recall their actions regarding care plans they had formulated at least a year ago. Nonetheless, PNs gave detailed answers to the questions in most instances, suggesting a small possibility of recall bias. A second limitation is that during the quantification process of parts of the semi-structured interviews, some numerical codes could not be inferred easily from the interview data due to a lack of detailed questioning by the interviewer or insufficient detail in answers given by PNs (see Table 5.2, 29.7\% of the reasons for not including a problem in the care plan are unknown). The use of an interview guide could not prevent that PNs often gave general case-related reasons instead of problem-specific reasons for not having included a problem in the care plan or for not having monitored the problem over time.

\section{CONCLUSION}

Despite high adherence of PNs to care plans, only about one quarter of the identified problems were included in a plan. The completeness of care plans varied between PNs and they experienced difficulties in monitoring older people over time. Additional training of PNs, adapting the care plan to fit PN's needs, and allowing PNs to monitor older people over time may improve the formulation of and adherence to care plans. Further research is needed to verify the current findings among a larger sample of PNs involved in proactive care for older people and by using objective record reviews instead of subjective accounts of PNs' actions. 


\section{REFERENCES}

1. Fried LP, Ferrucci L, Darer J, Williamson JD, Anderson G. Untangling the concepts of disability, frailty, and comorbidity: implications for improved targeting and care. J Gerontol A Biol Sci Med Sci 2004;59:255-263.

2. Lacas A, Rockwood K. Frailty in primary care: a review of its conceptualization and implications for practice. BMC Med 2012;10:4.

3. Laurant M, Reeves D, Hermens R, Braspenning J, Grol R, Sibbald B. Substitution of doctors by nurses in primary care. Cochrane Database Syst Rev 2004;4:Art. No.: CD001271.

4. Battersby M, Von Korff M, Schaefer J, Davis C, Ludman E, Greene SM, et al. Twelve evidence-based principles for implementing self-management support in primary care. Jt Comm J Qual Patient Saf 2010;36:561-570.

5. Stijnen MMN, Duimel-Peeters IGP, Jansen MWJ, Vrijhoef HJM. Early detection of health problems in potentially frail community-dwelling older people by general practices - project [G]OLD: design of a longitudinal, quasi-experimental study. BMC Geriatr 2013;13:7.

6. Huss A, Stuck AE, Rubenstein LZ, Egger M, Clough-Gorr KM. Multidimensional preventive home visit programs for community-dwelling older adults: a systematic review and meta-analysis of randomized controlled trials. J Gerontol A Biol Sci Med Sci 2008;63A:298-307.

7. Markle-Reid M, Browne G, Weir R, Gafni A, Roberts J, Henderson SR. The effectiveness and efficiency of home-based nursing health promotion for older people: a review of the literature. Med Care Res Rev 2006;63:531-569.

8. Stuck AE, Siu AL, Wieland GD, Adams J, Rubenstein LZ. Comprehensive geriatric assessment: a metaanalysis of controlled trials. Lancet 1993;342:1032-1036.

9. Reuben $\mathrm{DB}$, Roth $\mathrm{C}$, Kamberg C, Wenger NS. Reconstructing primary care practices to manage geriatric syndromes: the ACOVE-2 intervention. J Am Geriatr Soc 2003;51:1787-1793.

10. Metzelthin SF, Daniëls R, Van Rossum E, Cox K, Habets H, De Witte LP, et al. A nurse-led interdisciplinary primary care approach to prevent disability among community-dwelling frail older people: a large-scale process evaluation. Int J Nurs Stud 2013;50:1184-1196.

11. Edmonds WA, Kennedy TD. An applied reference guide to research designs. Quantitative, qualitative and mixed methods. Thousand Oaks, CA: Sage; 2013.

12. Dutch College of General Practitioners. NHG-Praktijkwijzer Ouderenzorg [Dutch College of General Practioners-Manual care for older people in general practice]. Utrecht: NHG, 2010.

13. Thomas DR. A general inductive approach for analyzing qualitative evaluation data. Am J Eval 2006;27:237-246.

14. Hegney DG, Patterson E, Eley DS, Mahomed R, Young J. The feasibility, acceptability and sustainability of nurse-led chronic disease management in Australian general practice: the perspectives of key stakeholders. Int J Nurs Pract 2013;19:54-59.

15. Stijnen MMN, Jansen MWJ, Vrijhoef HJM, Duimel-Peeters IGP. Development of a home visitation programme for the early detection of health problems in potentially frail community-dwelling older people by general practices. Eur J Ageing 2013;10:49-60.

16. Funkesson $\mathrm{KH}$, Anbäcken EM, Ek AC. Nurses' reasoning process during care planning taking pressure ulcer prevention as an example. A think-aloud study. Int J Nurs Stud 2007;44:1109-1119.

17. Lamb B, Sevdalis N. How do nurses make decisions? Int J Nurs Stud 2011;48:281-284.

18. Van Heuvelen MJG, Hochstenbach JBM, Brouwer WH, De Greef MHG, Zijlstra GAR, Jaarsveld E, et al. Differences between participants and non-participants in an RCT on physical activity and psychological interventions for older persons. Aging Clin Exp Res 2005;17:236-245.

19. Piccoliori G, Gerolimon E, Abholz H. Geriatric assessment in general practice using a screening instrument: is it worth the effort? Results of a South Tyrol Study. Age Ageing 2008;37:647-652. 
20. Evans C, Drennan V, Roberts J. Practice nurses and older people: a case management approach to care. J Adv Nurs 2005;51:343-352.

21. Boase S, Kim Y, Craven A, Cohn S. Involving practice nurses in primary care research: the experience of multiple and competing demands. J Adv Nurs 2011;68:590-599.

22. De Bakker DH, Struijs JN, Baan CA, Raams J, De Wildt J, Vrijhoef HJM, et al. Early results from adoption of bundled payment for diabetes care in the Netherlands show improvement in care coordination. Health Aff (Millwood) 2012;31:426-433. 

Early detection of health problems in potentially frail community-dwelling older people by general practices - project [G]OLD: design of a longitudinal, quasi-experimental study

Published as:

Stijnen MMN, Duimel-Peeters IGP, Jansen MWJ, Vrijhoef HJM. Early detection of health problems in potentially frail community-dwelling older people by general practices - project [G]OLD: design of a longitudinal, quasi-experimental study. BMC Geriatr, 2013;13:7. 


\section{ABSTRACT}

\section{Background}

Due to the ageing of the population, the number of frail older people who suffer from multiple, complex health complaints increases and this ultimately threatens their ability to function independently. Many interventions for frail older people attempt to prevent or delay functional decline, but they show contradicting results. Recent studies emphasise the importance of embedding these interventions into existing primary care systems and tailoring care to older people's needs and wishes. This article presents the design of an evaluation study, aiming to investigate the effects and feasibility of the early detection of health problems among community-dwelling older people and their subsequent referral to appropriate care and/or well-being facilities by general practices.

\section{Methods / design}

A longitudinal, quasi-experimental study is designed comparing 13 intervention practices with 11 control practices. General practices select eligible communitydwelling older people ( $\geq 75$ years). Practice nurses from intervention practices (1) visit older people at home for a comprehensive assessment of their health and well-being; (2) discuss results with the GP; (3) formulate - if required - a care and treatment plan together with the patient; (4) refer patient to care and/or well-being facilities; and (5) monitor and coordinate care and follow-up. Control practices provide usual care and match the intervention practices on the presence of different primary care professionals within the practice. Primary outcome measures are health-related quality of life and disability. Additionally, attitude towards ageing, care satisfaction, healthcare utilisation, nursing home admission and mortality are measured. Some outcomes are assessed by means of a postal questionnaire (at baseline and after 6,12 , and 18 months follow-up), others through continuous registration over the 18-month period. A profound process evaluation will provide insight into barriers and facilitators for implementing the intervention protocol within general practices from both the patient and caregiver perspective.

\section{Discussion}

The proposed approach requires redesigning care delivery within general practices for accomplishing appropriate care for older people. A quasi-experimental design is chosen to closely resemble a real-life situation, which is desirable for future implementation after this innovation proves to be successful. Results of the effect and process evaluation will become available in 2013 . 


\section{BACKGROUND}

Ageing of the population poses challenges to healthcare systems as the number of frail older people who suffer from complex and/or multiple (chronic) health complaints increases. ${ }^{1,2}$ A failure to detect health complaints among older people in time may cause unnecessary neediness and may threaten their ability to function independently.

Strategies comprising early identification of older people at risk of poor health and early intervention should prevent or postpone the onset of functional decline and maintain independent living. ${ }^{3}$ In the last decades, there has been an increased focus worldwide on the development of preventive home visitation programmes to support older people to grow old at home and to prevent or delay institutionalisation.

There is still an ongoing debate whether these preventive home visits should be part of regular care for older people. Numerous systematic reviews have been published, ${ }^{4-11}$ attempting to determine the effectiveness of preventive home visits, but the results remain inconclusive. Discrepancy in the results is caused, among others, by differences in the selection of the target population, intensity and duration of the intervention (i.e., number of follow-up visits), or domains included in the multidimensional assessment of older people's health status. ${ }^{12}$ Thus, the question remains which components of preventive home visits are effective and for which population they are beneficial. ${ }^{13}$ Most studies to date employ a randomised design for establishing the success of preventive home visits, thereby hindering close resemblance to a real-life situation and restricting the external validity of findings.

Recent publications stress the importance of integrating preventive interventions for older people into existing care systems. ${ }^{10,14,15}$ For example, Van Hout and colleagues ${ }^{14}$ attribute the absence of a preventive effect of home visits to the fact that they were not integrated within primary care practices. In our current approach, instead of solely integrating, we aim to redesign care delivery within primary care practices by applying components of the Chronic Care Model (CCM). This comprehensive framework has proven to lead to improved patient care and better health outcomes when changing routine delivery of care through improvements in six interrelated components (further details are provided in the Methods section). ${ }^{16,17}$ In addition, elements of the Guided Care model are incorporated in our approach. ${ }^{18}$ Guided Care used the CCM to identify successful innovations in chronic care that can be applied in primary care to achieve optimal outcomes in people with chronic diseases and complex care needs.

General practices seem to be the ideal setting for realising preventive care facilities for older people, because of their geographical proximity to older people, knowledge of the patient's medical history, relationship of trust between doctor and patient, and access to a range of multidisciplinary healthcare and well-being facilities in 
the person's neighbourhood. However, general practitioners (GPs) often do not have a complete overview of the health status and functioning of older people. ${ }^{19,20}$ A Dutch study among randomly selected older patients revealed that $34 \%$ of recorded health problems during a home visit were unknown to GPs (mostly psychosocial or physical complaints, such as depression and urine incontinence). ${ }^{19}$ Similarly, Alessi and coworkers $^{21}$ reported that three-quarter of the visited older people had at least one major health problem identified that was previously unknown. This suggests that a comprehensive geriatric assessment in the home setting yields important information about previously undetected health problems and this might be particularly beneficial for the apparently healthy older people.

It is equally important that older people themselves are aware of their own (unmet) health needs, as this appears to be supportive for maintaining independent living. ${ }^{22}$ It seems that older people tend to discard certain health problems or complaints as inevitable aspects of ageing, such as in the case of urinary incontinence, ${ }^{23}$ they forget about the occurrence of certain events, such as in reporting falling incidents, ${ }^{24}$ or they may fail to recognise the significance of symptoms or complaints. ${ }^{25} \mathrm{~A}$ multidimensional assessment may create awareness of these (unmet) needs or problems. After health problems and complaints are identified, care facilities should be tailored to older patient's needs and preferences ${ }^{26-28}$ and active involvement of older people in decision-making concerning their need for care services is encouraged. ${ }^{29}$

In conclusion, we hypothesise that a multidimensional comprehensive geriatric assessment of the health and well-being of community-dwelling older people by general practices and subsequent individualised care and follow-up (if required) will lead to improved health-related quality of life and reduced disability compared to usual care (i.e., reactive care to older people who present themselves with health problems or complaints). Furthermore, we hypothesise that this approach will be feasible from both the patient and caregiver perspective. In the current paper, the design of an evaluation study is presented aiming to investigate the effects of identifying health problems and complaints among potentially frail community-dwelling older people at an early stage and, if necessary, their follow-up within care and/or well-being facilities. Parallel to the effect study, a process evaluation will provide insight into the barriers and facilitators for implementing the proposed approach within general practices. 


\section{METHODS / DESIGN}

\section{Study design and setting}

The longitudinal, quasi-experimental study is performed in three regions in the south of the Netherlands: Maastricht-Heuvelland ( $8.5 \% \geq 75$ years), Parkstad $(8.7 \% \geq 75$ years), and Midden-Limburg $(7.5 \% \geq 75$ years). They are particularly interesting because the ageing of the population is more pronounced here (nationwide $7.0 \% \geq 75$ years). General practices in these regions were invited to participate in the evaluation study. Participating general practices randomly selected community-dwelling people aged 75 years and older from the GPs Information System. Older people within intervention practices are visited at home by the practice nurse for a multidimensional assessment followed by individualised care, the so-called [G]OLD-protocol: 'Getting OLD the healthy way'. Older people from control practices receive usual care (i.e., reactive care instead of proactive care). Effects on outcome measures are assessed at baseline $\left(T_{0}\right)$ and after 6-months $\left(T_{1}\right), 12$-months $\left(T_{2}\right)$, and 18-months $\left(T_{3}\right)$ follow-up. Parallel to the effect study, a process evaluation is performed. Figure 6.1 presents a flowchart of the study design and measurements. A more complete overview of the study protocol, including a time schedule, is provided in Figure 6.2.

The Medical Ethical Committee (MEC) of the Maastricht University Medical Centre (MUMC+) judged this evaluation study as not needing formal ethical approval. Nevertheless, the MEC granted their approval for our study protocol and informed consent documents.

\section{Selection of general practices}

General practices $(n=21)$ who visited older people at home as part of our pilot study ${ }^{30}$ were excluded from participation to prevent contamination of prior experience. We approached 188 general practices in the three regions for participation in this study. Practices in Midden-Limburg were only invited to participate in the control group, since insufficient general practices from the other two regions were willing to participate in the control group. GPs who indicated to be actively engaged in or are planning to start with the identification and follow-up of frail older people in a systematic way were ineligible to participate ( $n=12$ practices). The availability of a practice nurse who has time for care for older people is a prerequisite for intervention practices. Practice nurses work in general practices, and provide screening, treatment, care and education mainly to patients with chronic diseases and older people. 


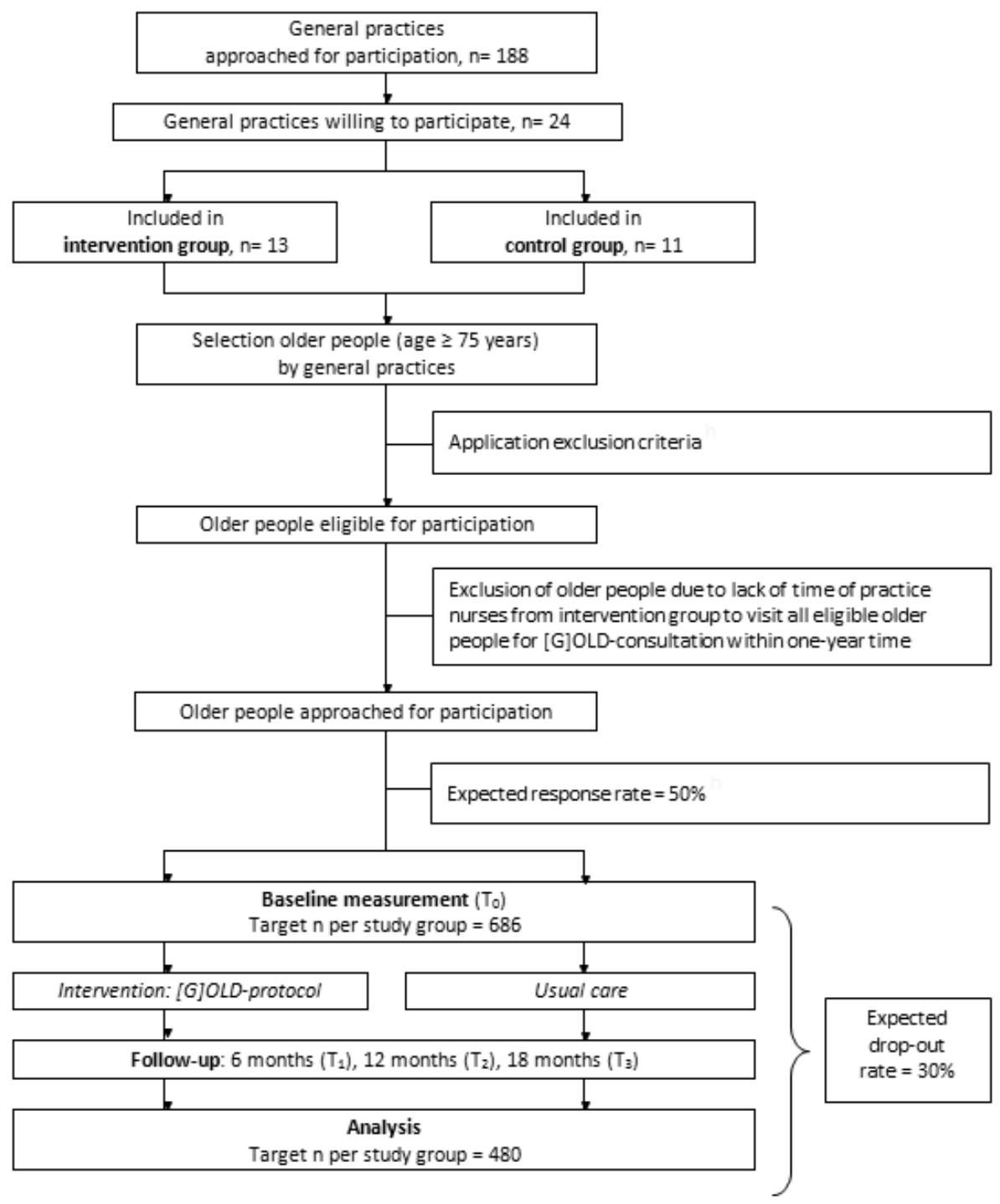

Figure 6.1 Flowchart study design and measurements

Reasons of general practices for non-participation were: no time (e.g., due to other priorities, staff changes or participation in other research projects) (35.0\%), no interest to participate in the present study (31.7\%), interested in [G]OLD-intervention but not in research (19.5\%), or no reason was mentioned (13.8\%).

Fourteen general practices agreed to participate as intervention practice and 13 general practices consented to participate in the control group. Control practices were matched to intervention practices based on the presence of primary care professionals 
within general practices to ensure comparability at baseline. We assume that close proximity of various primary care disciplines facilitates collaboration in organising and/or delivering appropriate care to older people. ${ }^{31}$ After the recruitment phase, one intervention practice and two control practices dropped out due to a lack of time to select older people eligible for participation. As a result, 24 general practices were included in this study.

\section{Target population}

The target population are the apparently healthy, community-dwelling older people aged 75 years and older. Although the age criterion causes much controversy, especially from the age of 75 years on the prevalence of frailty increases markedly. ${ }^{32}$ This enables us to find sufficient eligible older people for participation. Furthermore, some authors suggest that preventive home visits are most beneficial for people aged 75 years and older. ${ }^{33,34}$ We excluded people who are not living independently, those on a waiting list for admission to a nursing home or home for older people, those under close medical supervision (chemotherapy, chronic haemodialysis or other therapies posing a high burden on the person), and the terminally ill. Practice nurses' available working hours for care for older people determined the maximum number of older people that each of them was able to visit within one-year time. This, together with the size of the patient population aged 75 years and older, determined the number of older people per intervention practice approached for participation. In control practices, all eligible older people aged 75 years and over were approached.

We invited older people for participation by means of an information letter and consent form. We performed telephonic reminders in the intervention group and postal reminders after four weeks in the control group to those who did not respond to the first mailing.

\section{Procedure}

Although the independent effects of components of preventive home visitation programmes are difficult to disentangle, previous research has suggested elements that at least should be included, such as a comprehensive geriatric assessment, a concrete care plan and multiple follow-up contacts. ${ }^{7,} 8,29$ We redesigned care delivery for older people by general practices by focusing on several evidence-based elements of the Chronic Care Model (CCM) and the Guided Care model (for details, see Figure 6.2). Applying both models has led to the development of the [G]OLD-protocol, which is explained in more detail below. Our pilot study provided preliminary evidence of the feasibility of the [G]OLD-protocol for general practices. ${ }^{30}$ 


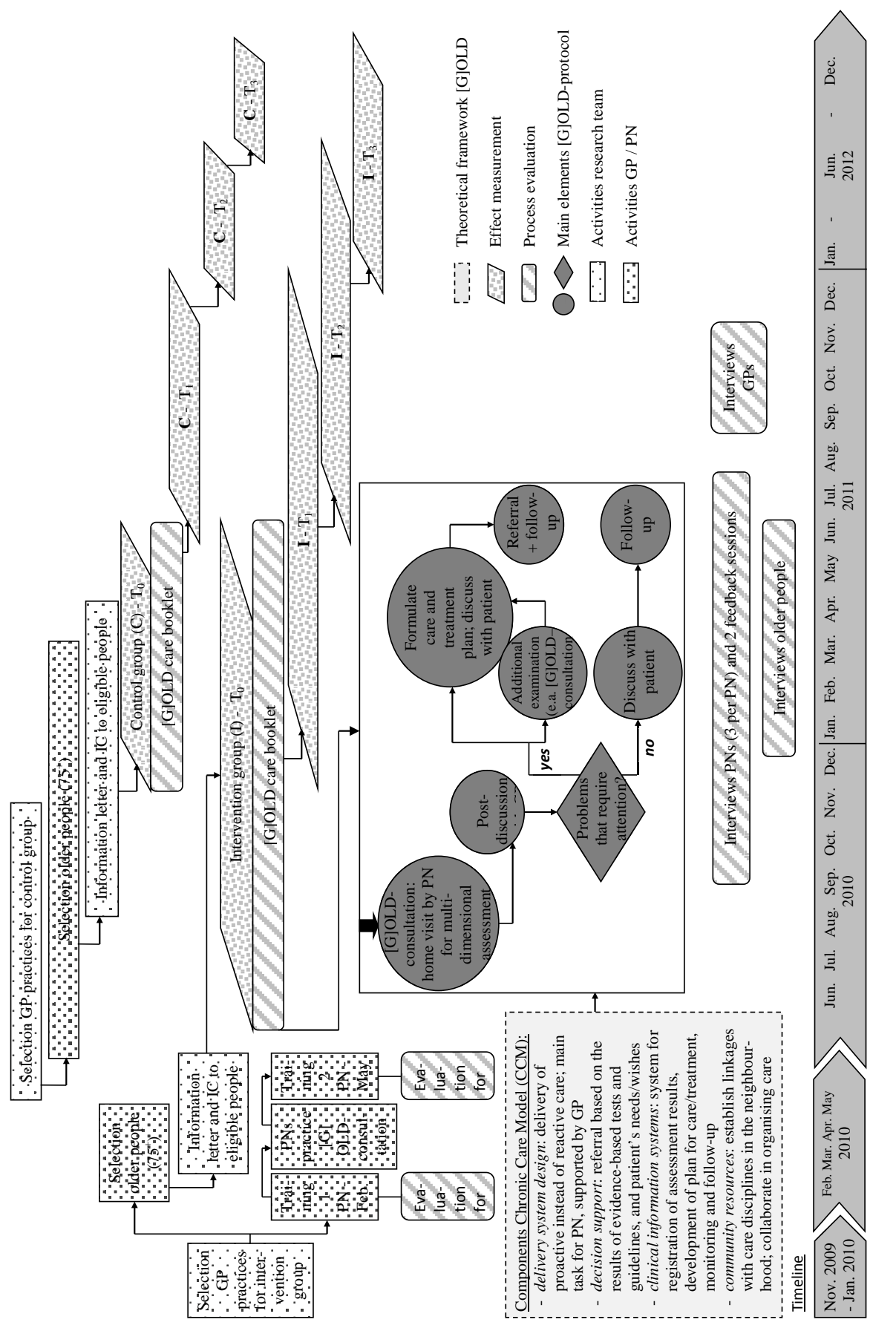

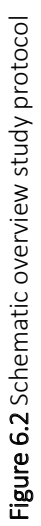




\section{Training}

Practice nurses from intervention practices received two days of training before the start of the study to provide them with the necessary knowledge and skills for executing all elements of the [G]OLD-protocol. In this way, we also attempted to equalise the level of knowledge and skills between practice nurses regarding care for older people. Central elements of the training included acquiring communication skills, gaining knowledge about frequently occurring health problems among older people, gaining knowledge about health services for older people, and learning how to assess older people's physical, psychological, mental and social functioning by means of a multidimensional instrument. In between the two training sessions, each practice nurse performed exactly five home visits among randomly chosen older people ( $\geq 75$ years) during a try-out phase. During the intervention period, sessions were organised for asking questions and exchanging experiences, and practice nurses received additional support by a coach specialised in geriatric care.

\section{Home visit - comprehensive geriatric assessment}

The practice nurse invites older people for a home visit successively within one year of time. Before the visit, the practice nurse makes a print out of the person's medication list and medical history for relevant details or major events to be aware of.

During the visit, the practice nurse uses the [G]OLD-instrument: a structured, comprehensive geriatric assessment to assess the person's physical, psychological, mental and social functioning, as well as lifestyle and medication use (see Table 6.1). This instrument is specifically developed for and tested among the apparently healthy community-dwelling older people aged 75 years and older in a pilot study. ${ }^{30}$ Suggestions made during the pilot phase helped to improve the [G]OLD-instrument for application in the current longitudinal, quasi-experimental study. In general, the instrument assists the practice nurse in uncovering (early signs of) potential health problems or needs that may prevent older people, now or in the near future, from maintaining independent living. Although the instrument follows a structured format, it can be applied in a flexible way. For each test included, evidence-based cut-off points and guidelines are presented to assist in deciding about the presence or absence of health problems or needs.

Crucial during the visit is establishing a relationship of trust, listening to the needs and wishes of the older person, and allowing the person time to talk. ${ }^{29}$ If necessary, the practice nurse may also provide information or advice. Sometimes it is necessary to perform an additional examination to obtain a more accurate estimation of the presence of problems. Therefore, more elaborate tests on the themes cognition, depression and personality disorders are incorporated in the [G]OLD-instrument part 2 
which can be conducted during the first visit or during a second visit, depending on the older person's preference.

After the home visit, the practice nurse registers the results of the [G]OLDinstrument in the electronic patient file.

Table 6.1 Topics included in the [G]OLD comprehensive geriatric assessment instrument

\begin{tabular}{|c|c|c|}
\hline & $\begin{array}{l}\text { Basic assessment } \\
\text { - part one }\end{array}$ & $\begin{array}{l}\text { Additional assessment } \\
\text { - part two }\end{array}$ \\
\hline $\begin{array}{l}\text { Physical } \\
\text { functioning and } \\
\text { lifestyle }\end{array}$ & $\begin{array}{l}\text { Disability in ADL and IADL; need for assistance in ADL and/or } \\
\text { IADL; incontinence; mobility; falls; vision and hearing problems; } \\
\text { BMI (height and weight); malnutrition; blood pressure; physical } \\
\text { activity; smoking; alcohol use }\end{array}$ & N/A \\
\hline $\begin{array}{l}\text { Psychological } \\
\text { functioning }\end{array}$ & Cognition; anxiety; depression; personality disorders & $\begin{array}{l}\text { Cognition; depression; } \\
\text { personality disorders }\end{array}$ \\
\hline Social functioning & $\begin{array}{l}\text { Receiving and providing informal care; loneliness; social } \\
\text { participation }\end{array}$ & N/A \\
\hline Additional & $\begin{array}{l}\text { General perception of health and quality of life; medication use; } \\
\text { financial situation; healthcare utilisation; observation of living } \\
\text { environment; physical, psychological and behavioural signals }\end{array}$ & N/A \\
\hline
\end{tabular}

Note: N/A means not applicable.

\section{Post-discussion GP and formulating care and treatment plan}

The practice nurse discusses the results of the home visit with the GP. The results of the [G]OLD-instrument, as well as the patient's needs and wishes, determine whether follow-up actions regarding certain problems are needed. These actions may consist of additional diagnosis, preventive care or advise, treatment in primary healthcare or referral to other care and/or well-being facilities as much as possible in the older person's neighbourhood. The practice nurse formulates a provisional individualised care and treatment plan. This plan is discussed with the patient, whose input and wishes lead to a final care and treatment plan, which is registered in the electronic patient file.

\section{Referral and follow-up}

The practice nurse arranges and coordinates care for the older person as formulated in the final care and treatment plan and monitors the follow-up. The need for and frequency of follow-up contacts strongly depends on the type of problems or complaints that deserve attention according to the care and treatment plan. Hence, no fixed number of contacts per older person is determined on forehand. The practice nurse indicates in the care and treatment plan at what date a specific problem or complaint will be re-evaluated. Then, at each follow-up contact, the need for additional follow-up 
contacts is determined and, if necessary, the care and treatment plan is adjusted. Notably, these follow-up contacts may also take place with other care providers to whom older people are being referred.

If follow-up actions are not required or they are not desirable from the patient's point of view, the practice nurse discusses with both the GP and the older person how they will proceed from that moment on. It is important that general practices should prevent to lose sight of their older patients after this initial assessment. The home visit is not meaningless when no specific problems are identified, as it helps to gain knowledge about the patient in case future health problems occur (e.g., falling incidents). Furthermore, the bond created with the practice nurse increases the likelihood that older people will approach their general practice in case of any future problems or complaints. Older people who do not receive follow-up contacts will remain part of the study population to ensure comparability with the control group and they will be analysed as a sub-group.

\section{Measures and data collection}

The primary outcome measures in this study are health-related quality of life measured by the RAND-36 $6^{35,36}$ and disability in activities of daily living (ADL, including mobility) and instrumental activities of daily living (IADL), assessed using the Groningen Activity Restriction Scale (GARS). ${ }^{37}$ Both instruments appear to be valid, reliable and suitable for self-completion in older people. ${ }^{38,39}$ These outcomes, together with the secondary outcome attitude towards ageing (subscale attitude toward ageing from the PGC Morale Scale ${ }^{40}$ are included in a questionnaire send to older people by postal mail at baseline, 6-months, 12-months and 18-months follow-up. The baseline questionnaire also gathers data about socio-demographic variables (i.e., age, gender, ethnicity, educational level, marital status, household composition) to provide insight into characteristics of the target population. Assistance is provided to older people who are unable to self-complete the questionnaires or those with many missing items (mostly people with poor physical or mental health).

Additional secondary outcomes are admission to a nursing home or home for older people, healthcare utilisation, and mortality. General practices register these outcomes continuously during the study period in the GP's Information System and data are extracted for each patient after 18-months follow-up. Furthermore, healthcare utilisation is also recorded in the [G]OLD care booklet. Older people receive this booklet at baseline and are requested to take it with them to each contact with professional healthcare providers for 18 months. In this booklet, patients and/or care providers indicate the reason for the contact, type of health problems or complaints, 
and follow-up activities. Table 6.2 presents all outcome measures, their operationalisation and timing of data collection.

Table 6.2 Measures, operationalisation and timing of data collection

\begin{tabular}{|c|c|c|c|c|}
\hline Measures & Operationalisation & $\begin{array}{l}\text { No. of } \\
\text { items }\end{array}$ & $\begin{array}{l}\text { Range } \\
\text { score* }\end{array}$ & $\begin{array}{l}\text { Timing data } \\
\text { collectiont }\end{array}$ \\
\hline \multicolumn{5}{|l|}{ Primary outcomes } \\
\hline Health-related quality of life & RAND-36 35,36 & 36 & N/A & $\mathrm{T}^{0}, \mathrm{~T}^{1}, \mathrm{~T}^{2}, \mathrm{~T}^{3}$ \\
\hline \multirow[t]{3}{*}{ Disability } & $\mathrm{GARS}^{37}$ & 18 & $\underline{18-72}$ & $T^{0}, T^{1}, T^{2}, T^{3}$ \\
\hline & IADL & 11 & 11-44 & \\
\hline & ADL & 7 & $\underline{7}-28$ & \\
\hline \multicolumn{5}{|l|}{ Secondary outcomes } \\
\hline Attitude towards ageing & $\begin{array}{l}\text { Subscale attitude toward own ageing - } \\
\text { PGC Morale Scale }\end{array}$ & 5 & $0-\underline{5}$ & $\mathrm{~T}^{0}, \mathrm{~T}^{1}, \mathrm{~T}^{2}, \mathrm{~T}^{3}$ \\
\hline Healthcare utilisation & $\begin{array}{l}\text { Number of contacts with different } \\
\text { healthcare providers (i.e., GP } \\
\text { consultations, hospital admission) }\end{array}$ & $\begin{array}{c}3 \\
N / A\end{array}$ & $\begin{array}{l}\text { N/A } \\
\text { N/A }\end{array}$ & $\begin{array}{c}\mathrm{T}^{0} \\
\text { CR_GP and CR_E }\end{array}$ \\
\hline $\begin{array}{l}\text { Admission to nursing home } \\
\text { or home for older people }\end{array}$ & $\begin{array}{l}\text { Number of admissions and time to } \\
\text { admission from } \mathrm{T}^{0} \text { to } \mathrm{T}^{3}\end{array}$ & N/A & N/A & CR_GP \\
\hline Mortality & Number of deaths from $\mathrm{T}^{0}$ to $\mathrm{T}^{3}$ & N/A & N/A & CR_GP \\
\hline
\end{tabular}

* Underlined scores indicate the most favourable scores. N/A means not applicable.

$+\mathrm{T}_{0}=$ postal questionnaire at the start of the study; $\mathrm{T} 1$ = postal questionnaire at 6-months follow-up; $\mathrm{T} 2=$ postal questionnaire at 12-months follow-up; T3 = postal questionnaire at 18-months follow-up; CR_GP = continuous registration during study in GP's Information System; CR_E = continuous registration during study by older people in [G]OLD care booklet

\section{Process evaluation}

A thorough process evaluation is conducted aiming to investigate to what extent the different components of the [G]OLD-protocol are implemented within general practices as planned (e.g., barriers and facilitators for implementation) and the feasibility of the protocol for both patients and caregivers. Ultimately, the results may provide information for further implementation of [G]OLD within general practices. Qualitative and quantitative process data are collected with either formative or summative purposes among GPs, practice nurses and older people according to the comprehensive and systematic approach proposed by Saunders and colleagues ${ }^{41}$ (for details, see Table 6.3). 
Table 6.3 Data collection as part of the process evaluation

\begin{tabular}{|c|c|c|}
\hline Components & Operationalisation & Data collection tools/procedures \\
\hline $\begin{array}{l}\text { Fidelity } \\
\text { (quality) }\end{array}$ & $\begin{array}{l}\text { Extent to which the [G]OLD-protocol was } \\
\text { implemented as planned }\end{array}$ & $\begin{array}{l}\text { Evaluation form training PNs } \\
\text { Individual interviews PNs, GPs and older } \\
\text { people } \\
\text { Feedback sessions PNs } \\
\text { [G]OLD-instrument and care and } \\
\text { treatment plan }\end{array}$ \\
\hline $\begin{array}{l}\text { Dose delivered } \\
\text { (completeness) }\end{array}$ & $\begin{array}{l}\text { Extent to which all aspects of [G]OLD- } \\
\text { protocol are delivered to general practices } \\
\text { and older people }\end{array}$ & $\begin{array}{l}\text { Evaluation form training PNs } \\
\text { Individual interviews PNs, GPs and older } \\
\text { people } \\
\text { Feedback sessions PNs } \\
\text { [G]OLD-instrument and care and } \\
\text { treatment plan }\end{array}$ \\
\hline $\begin{array}{l}\text { Dose received } \\
\text { (exposure) }\end{array}$ & $\begin{array}{l}\text { Extent to which PNs, GPs and older people } \\
\text { actively engage in, interact with and are } \\
\text { receptive to aspects of [G]OLD-protocol }\end{array}$ & $\begin{array}{l}\text { Individual interviews PNs, GPs and older } \\
\text { people } \\
\text { Feedback sessions PNs } \\
\text { [G]OLD care booklet for older people }\end{array}$ \\
\hline $\begin{array}{l}\text { Dose received } \\
\text { (satisfaction) }\end{array}$ & $\begin{array}{l}\text { Overall opinion of PNs, GPs, and older } \\
\text { people about [G]OLD }\end{array}$ & $\begin{array}{l}\text { Evaluation form training PNs } \\
\text { Individual interviews } \\
\text { Feedback sessions PNs } \\
\text { [G]OLD care booklet for older people }\end{array}$ \\
\hline $\begin{array}{l}\text { Reach } \\
\text { (participation rate) }\end{array}$ & $\begin{array}{l}\text { Proportion of intended target population } \\
\text { that participates in and completes the } \\
\text { intervention: } \\
\text { (1) registration number and reasons for } \\
\text { non-response and drop-out; (2) opinion } \\
\text { PNs and GPs about reach }\end{array}$ & $\begin{array}{l}\text { Continuous registration by general } \\
\text { practices and researchers } \\
\text { Individual interviews with PNs and GPs }\end{array}$ \\
\hline Context & $\begin{array}{l}\text { Environmental barriers and facilitators } \\
\text { that influence implementation [G]OLD, } \\
\text { continued involvement in [G]OLD, and/or } \\
\text { study outcomes }\end{array}$ & $\begin{array}{l}\text { Individual interviews with PNs and GPs } \\
\text { Notes researchers and project leaders }\end{array}$ \\
\hline
\end{tabular}

Note: $\mathrm{PN}=$ practice nurse; $\mathrm{GP}=$ general practitioner 
The experience of practice nurses with the [G]OLD-protocol is assessed three times during one year (intervention period) by means of individual interviews. Results of these interviews were fed back to all practice nurses together during feedback sessions after six months and after one year (end of the intervention period). Additionally, GPs were individually interviewed at the end of the intervention period to assess their experiences with the implementation of [G]OLD within their general practice. One older person per general practice was selected for in-depth interviews about their experiences and satisfaction with all aspects of the [G]OLD-protocol, approximately one month after the [G]OLD-consultation took place. Furthermore, older people can register their satisfaction with contacts with professional care givers in the [G]OLDcare booklet. Finally, time required for the home visit, results of the tests performed and preliminary advise given to people during the home visit are registered by the practice nurse on the [G]OLD-instrument. Details about referral to care and/or wellbeing services are written down in the care and treatment plan. Members of the research team checked monthly during the intervention period to what extent the [G]OLD-instrument and the care and treatment plan were completely filled out. Practice nurses registered the patient's follow-up within the chain of care in the electronic patient file from which relevant data can be extracted after 18-months follow-up.

\section{Sample size considerations}

The sample size calculation is based on the primary outcome measure health-related quality of life (subscale 'general health perception') as measured by the RAND-36. ${ }^{35,36}$ We aim to demonstrate a clinically relevant difference between the mean change score of the intervention and control group of 5.0 on the transformed subscale. This implies a standardised effect size of 0.24 (given SD $=21.2$ ). Based on this and applying a significance level $(\alpha)$ of 0.05 and a power of 0.90 , the minimally required number of participants is 564 ( $n=282$ per study group) using an independent samples t-test (twosided). However, calculations that take into account the interdependency of the measurements within a cluster (i.e., general practice) and correct for the cluster effect result in a required sample size of 960 older people ( $n=480$ per study group).

We expected a response rate of $50 \%$ on the information letter and consent form sent to eligible older people for participation and a drop-out rate of $30 \%$. Accounting for drop-out, we planned to enrol 686 older people per study group to have a sufficient number of participants per group (480 older people) at the end of 18 months follow-up (see also Figure 6.1). Because of the expected response rate of $50 \%$, we planned to approach at least 1,371 community-dwelling older people per study group for participation. 
Since the amount of home visits that is performed depends on the PNs available time, we expected a variation in cluster sizes. This is compensated for by sampling $25 \%$ more clusters (i.e., general practices). ${ }^{42}$

\section{Statistical analyses}

We compute descriptive statistics to describe the characteristics of the target population and general practices and to investigate comparability of study groups at baseline. Relevant statistical tests (e.g., t-test, chi-square, analysis of variance, regression analysis) will be applied to analyse effects on primary and secondary outcome measures (level of significance is 0.05 ; two-tailed). Data will be analysed according to the intention-to-treat principle. In all analyses, there will be a correction for possible baseline differences between participants or general practices. In addition, we will perform subgroup analyses to investigate whether certain groups of older people benefit more from the [G]OLD-protocol than other groups. We will use the software package SPSS for Windows, version 17.0, for all statistical analyses.

Data gathered as part of the process evaluation will be analysed using descriptive techniques, such as calculating scores (e.g., number of drop-outs), narrative description of procedures, and identifying themes in the interviews.

\section{DISCUSSION}

In the present paper, the design of a longitudinal, quasi-experimental study is presented to investigate the effects of the early detection of health problems among community-dwelling older people and their subsequent follow-up within the chain of care by general practices. In contrast to existing studies, we purposefully chose for a quasiexperimental design. Although randomised controlled trials are widely accepted as the "gold standard" for evaluating the effectiveness of interventions, they create artificial situations that may hinder the translation of research findings into practice. ${ }^{43-45}$ Moreover, the study may suffer from the uncertain commitment of the people delivering the intervention (in this case the general practice's staff) to the changes to be made. Routinisation of working methods in daily practice must take place to ensure sustainability of the [G]OLD-protocol, ${ }^{46}$ which is more difficult to realise within a randomised design. Our combination of an effect study and a thorough process evaluation should provide sufficient information with respect to the feasibility and external validity of the [G]OLD-protocol within general practices.

Furthermore, we predominantly used the Chronic Care Model for redesigning primary care practice as applying elements of this framework appears to lead to im- 
proved patient care and better health outcomes among patients. ${ }^{17}$ We additionally expect that the multidimensional [G]OLD-instrument will be of added value in providing a comprehensive overview of the older person's health status, compared to intervention programs that only focus on a limited number of tests or questions in only one or two domains.

Challenges faced during the intervention period are managing internal and external factors (e.g., changes in the general practice's policy or reimbursement of medical expenses by insurance companies) to allow for continued and adequate implementation. Furthermore, considering the current interest of general practices in care for older people, general practices who participate in the control group are closely monitored until the end of the follow-up period to find out if they implement any activities that are similar to practices applying the [G]OLD-protocol. They may undertake initiatives that improve their care for older people and this may distort the intervention effect. Also, several factors may influence the extent to which general practices are successfully redesigned, such as the influence of existing routines and the care providers own clinical opinion (or "gut feelings") on medical decision-making and referring older patients to adequate care and/or well-being facilities. Although we provided the necessary guidelines and recommendations to facilitate this process, GPs and practice nurses may not have ignored their own medical expertise in deciding about the diagnosis of health problems and/or referral and follow-up. The process evaluation will provide insight in the extent to which general practices redesign their care delivery to older people according to the [G]OLD-protocol. Results of the effect and process evaluation will become available in 2013. 


\section{REFERENCES}

1. Blokstra A, Baan CA, Boshuizen HC, Feenstra TL, Hoogenveen RT, Picavet HSJ, et al. Vergrijzing en toekomstige ziektelast. Prognose chronische ziektenprevalentie 2005-2025 [Impact of the ageing population on burden of disease. Projections of chronic disease prevalence for 2005-2025] Bilthoven: RIVM, 2007.

2. Christensen K, Doblhammer G, Rau R, Vaupel JW. Ageing populations: the challenges ahead. Lancet 2009;374:1196-1208.

3. Hébert R. Functional decline in old age. Can Med Assoc J 1997;157:1037-1045.

4. Stuck AE, Siu AL, Wieland GD, Adams J, Rubenstein LZ. Comprehensive geriatric assessment: a metaanalysis of controlled trials. Lancet 1993;342:1032-1036.

5. Van Haastregt JCM, Diederiks JPM, Van Rossum E, De Witte LP, Crebolder HFJM. Effects of preventive home visits to elderly people living in the community: systematic review. Br Med J 2000;320:754-758.

6. Elkan R, Kendrick D, Dewey M, Hewitt M, Robinson J, Blair M, et al. Effectiveness of home based support for older people: systematic review and meta-analysis. Br Med J 2001;323:719-725.

7. Stuck AE, Egger M, Hammer A, Minder CE, Beck JC. Home visits to prevent nursing home admission and functional decline in elderly people: systematic review and meta-regression analysis. J Am Med Assoc 2002;287:1022-1029.

8. Markle-Reid M, Browne G, Weir R, Gafni A, Roberts J, Henderson SR. The effectiveness and efficiency of home-based nursing health promotion for older people: a review of the literature. Med Care Res Rev 2006;63:531-569.

9. Perry M, Mokkink HGA. Is het zinvol om zelfstandig wonende ouderen systematisch te bezoeken? [Is it useful to systematically visit independently living elderly people?]. Tijdschr Prakt 2006;1:81-89.

10. Huss A, Stuck AE, Rubenstein LZ, Egger M, Clough-Gorr KM. Multidimensional preventive home visit programs for community-dwelling older adults: a systematic review and meta-analysis of randomized controlled trials. J Gerontol A Biol Sci Med Sci 2008;63A:298-307.

11. Bouman A, Van Rossum E, Nelemans P, Kempen GIJM, Knipschild P. Effects of intensive home visiting programs for older people with poor health status: a systematic review. BMC Health Serv Res 2008;8:74.

12. Rubenstein LZ, Stuck AE. Preventive home visits for older people: defining criteria for success. Age Ageing 2001;30:107-109.

13. Egger M. Commentary: when, where, and why do preventive home visits work? $\mathrm{Br} \mathrm{Med} J$ 2001;323:724-725.

14. Van Hout HPJ, Jansen APD, Van Marwijk HWJ, Nijpel G. Prevention of adverse health trajectories in a vulnerable elderly population through nurse home visits: randomized controlled trial [ISRCTN05358495]. J Gerontol A Biol Sci Med Sci 2010;65:734-742.

15. Dutch College of General Practitioners. NHG-Standpunt Toekomstvisie Huisartsenzorg. Huisartsgeneeskunde voor ouderen [Statement of the NHG, Future of General Practitioner Care. General practice medicine for older people]. Utrecht: NHG, 2007.

16. Bodenheimer T, Wagner EH, Grumbach K. Improving primary care for patients with chronic illness. J Am Med Assoc 2002;288:1775-1779.

17. Coleman K, Austin BT, Brach C, Wagner EH. Evidence on the chronic care model in the new millennium. Health Aff (Millwood) 2009;28:75-85.

18. Boult C, Karm L, Groves C. Improving chronic care: the "Guided Care" model. Perm J 2008;12:50-54.

19. Stalenhoef PA, Huijnen LGJ, Schonck RSM, Knottnerus JA, Van der Horst FG, Crebolder HFJM. Huisbezoek door de wijkverpleegkundige kan bijdragen aan de kennis van de huisarts over oudere patiënten. [Home visits by community nurses may contribute to general practitioner's information about elderly patients]. Ned Tijdschr Geneeskd 1996;140:2464-2467. 
20. Wilson CC, Netting FE. Comparison of self and health professionals' ratings of the health of communitybased elderly. Int J Aging Hum Dev 1987;25:11-25.

21. Alessi CA, Stuck AE, Aronow HU, Yuhas KE, Bula CJ, Madison R, et al. The process of care in preventive in-home comprehensive geriatric assessment. J Am Geriatr Soc 1997;45:1044-1050.

22. Chen Y-M, Thompson EA. Understanding factors that influence success of home- and community-based services in keeping older adults in community settings. J Aging Health 2010;22:267-291.

23. Teunissen D, Van Weel C, Lagro-Janssen T. Urinary incontinence in older people living in the community: examining help-seeking behaviour. Br J Gen Pract 2005;55:776-782.

24. Cummings SR, Nevitt MC, Kidd S. Forgetting falls. The limited accuracy of recall of falls in the elderly. J Am Geriatr Soc 1988;36:613-616.

25. Bender P. Deceptive distress in the elderly. Am J Nurs 1992;92:28-32.

26. Beswick AD, Rees K, Dieppe P, Ayis S, Gooberman-Hill R, Horwood J, et al. Complex interventions to improve physical function and maintain independent living in elderly people: a systematic review and meta-analysis. Lancet 2008;371:725-735.

27. Beswick AD, Gooberman-Hill R, Smith A, Wylde V, Ebrahim S. Maintaining independence in older people. Rev Clin Gerontol 2010;20:128-153.

28. Fagerström L, Wikblad A, Nilsson J. An integrative research review of preventive home visits among older people - is an individual health resource perspective a vision or a reality? Scand J Caring Sci 2008;23:558-568.

29. Vass M, Avlund K, Hendriksen C, Philipson L, Riis P. Preventive home visits to older people in Denmark. Why, how, by whom, and when? Z Gerontol Geriatr 2007;40:209-216.

30. Stijnen MMN, Jansen MWJ, Vrijhoef HJM, Duimel-Peeters IGP. Development of a home visitation programme for the early detection of health problems in potentially frail community-dwelling older people by general practices. Eur J Ageing 2013;10:49-60.

31. Lemieux-Charles L, McGuire WL. What do we know about health care team effectiveness? A review of the literature. Med Care Res Rev 2006;63:263-300.

32. Rockwood K, Howlett SE, MacKnight C, Beattie BL, Bergman H, Hébert R, et al. Prevalence, attributes, and outcomes of fitness and frailty in community-dwelling older adults: report from the Canadian Study of Health and Aging J Gerontol A Biol Sci Med Sci 2004;59:1310-1317.

33. Newbury J, Marley J. Preventive home visits to elderly people in the community. Visits are most useful for people aged >75. Br Med J 2000;321:512.

34. Stuck AE, Beck JC, Egger M. Preventing disability in elderly people Lancet 2004;364:1641-1642.

35. Van der Zee KI, Sanderman R. Het meten van de algemene gezondheidstoestand met de RAND-36: een handleiding [Measurement of general health status with the RAND-36: a guideline]. Groningen: Rijksuniversiteit Groningen, Noordelijk Centrum voor Gezondheidsvraagstukken, 1993.

36. Hays RD, Sherbourne CD, Mazel RM. The RAND 36-Item Health Survey 1.0. Health Econ 1993;2:217227.

37. Kempen GIJM, Doeglas DM, Suurmeijer TPBM. Het meten van problemen met zelfredzaamheid op verzorgend en huishoudelijk gebied met de Groningen Activiteiten Restrictie Schaal (GARS): een handleiding [Measurement of disability in activities of daily living and instrumental activities of daily living with the Groning Activity Restriction Scale (GARS): a guideline]. Groningen: Rijksuniversiteit Groningen, Noordelijk Centrum voor Gezondheidsvraagstukken, 1993.

38. Kempen GIJM, Miedema I, Ormel J, Molenaar W. The assessment of disability with the Groningen Activity Restriction Scale. Conceptual framework and psychometric properties. Soc Sci Med 1996;33:1601-1610.

39. Hayes V, Morris J, Wolfe C, Morgan M. The SF-36 health survey questionnaire: is it suitable for use with older adults? Age Ageing 1995;24:120-125.

40. Lawton MP. Lawton's PGC Morale Scale 2003 [cited 2009 November 10]. Available from: http://www.abramsoncenter.org/pri/documents/PGC_morale_scale.pdf. 
41. Saunders RP, Evans MH, Joshi P. Developing a process-evaluation plan for assessing health promotion program implementation: a how-to guide. Health Promot Pract 2005;6:134-147.

42. Van Breukelen GJP, Candel MJJM, Berger MPF. Relative efficiency of unequal versus equal cluster sizes in cluster randomized and multicentre trials. Stat Med 2007;26:2589-2603.

43. Green LW, Glasgow RE. Evaluating the relevance, generalization, and applicability of research. Issues in external validation and translation methodology Eval Health Prof 2006;29:126-153.

44. Glasgow RE, Green LW, Klesges LM, Fisher E, Goldstein MG, Hayman LL, et al. External validity: we need to do more. Ann Behav Med 2006;31:105-108.

45. Green LW. Making research relevant: if it is an evidence-based practice, where's the practice-based evidence? Fam Pract 2008;25:20-24.

46. Jansen M, Harting J, Ebben N, Kroon B, Stappers J, Van Engelshoven E, et al. The concept of sustainability and the use of outcome indicators. A case study to continue a successful health counselling intervention. Fam Pract 2008;25:32-37. 

Effectiveness of a nurse-led home visitation programme on health-related quality of life and disability among potentially frail communitydwelling older people in primary care: a longitudinal, quasi-experimental study

Stijnen MMN, Vrijhoef HJM, Jansen MWJ, Candel MJJM, Duimel-Peeters IGP. Effectiveness of a nurse-led home visitation programme on health-related quality of life and disability among potentially frail community-dwelling older people in primary care: a longitudinal, quasi experimental study. 


\section{ABSTRACT}

\section{Background}

General practices are confronted with organising care for the growing number of older people who suffer from multiple, complex health and well-being problems that threaten independent living.

\section{Objectives}

To investigate the effectiveness of the [G]OLD home visitation programme for potentially frail community-dwelling older people (aged $\geq 75$ years), consisting of a comprehensive geriatric assessment, formulation of a care and treatment plan, multidisciplinary care management, and targeted intervention and follow-up by general practices.

\section{Methods}

A cluster quasi-experimental trial in 24 general practices in the south of the Netherlands. Thirteen practices implemented the intervention, whereas eleven practices provided usual care. Community-dwelling older people aged $\geq 75$ years were eligible to participate. Primary outcome measures were health-related quality of life and disability. The secondary outcome was attitude towards ageing. Outcomes were assessed with self-reported questionnaires at baseline, 6,12 , and 18 months followup.

\section{Results}

In total, 1,527 potentially frail older people were included. Their mean age was 81.07 years (SD=4.05), $56.0 \%$ was female. 571 out of 586 participants $(97.4 \%)$ received the intervention and 941 participants received usual care. Mixed regression models only showed a small but significant difference in favour of the intervention group for role limitations due to physical problems at 6 months and 18 months follow-up.

\section{Conclusion}

Limited evidence was found for the effectiveness of the [G]OLD home visitation programme compared to usual care. Therefore, continuation of the intervention in its current form should be carefully reconsidered. Existing intervention models require continuous improvement and evaluation while implemented in the intended context. 


\section{INTRODUCTION}

Worldwide, healthcare professionals are confronted with the complex task of organising care for the expanding population of older people who often present themselves with multiple and coexisting health problems. ${ }^{1,2}$ As people grow old, they are also increasingly susceptible to become frail, ${ }^{3}$ caused by age-related decline in physiological systems which makes individuals vulnerable to sudden health changes triggered by minor events. ${ }^{4}$ As a result, older people are at increased risk of adverse outcomes such as disability, functional decline, and poor health-related quality of life, ${ }^{1,4}$ which threatens their ability to maintain independent living. Previous studies suggest that interdisciplinary, multifactorial interventions with tailored care and long-term follow-up can prevent or reduce the adverse effects of ageing in community-dwelling older people. ${ }^{5,6}$ These elements are often embedded in preventive home visitation programmes, although content alone is no guarantee for success. Preventive home visits for community-dwelling older people exist for many years and are part of routine care delivery in for example the United Kingdom ${ }^{7}$ and Denmark, ${ }^{8}$ yet reviews on their effectiveness show conflicting results. ${ }^{9-13}$ The latest systematic review concludes that preventive home visits are not consistently associated with effects on mortality and independent living, although some individual programmes may produce beneficial outcomes. ${ }^{11}$ It is proposed that future studies should incorporate a clear theoretical foundation underlying the intervention to disentangle components that may yield beneficial outcomes. ${ }^{11,14}$ Furthermore, it is advocated to embed home visitation programmes into general practice, ${ }^{15,16}$ because General Practitioners (GPs) are geographically close to older people, have knowledge of the person's medical history, and they are familiar with a patient-centred approach to care delivery. ${ }^{17}$ Consequently, GPs are challenged with delivering individually appropriate, proactive care to older people instead of reactively addressing health problems according to a disease management approach. ${ }^{18}$ In the Netherlands and other industrialised countries, expansion of primary care to meet the emerging needs of chronically ill patients and older people resulted in shifting of care from GPs to practice nurses (PNs) with a professional nursing background. ${ }^{19}$ They are involved in, among others, disease prevention, chronic care management, and care for frail older people ${ }^{20}$ and they can realise as high quality care and as good health outcomes as GPs. ${ }^{19}$

Above reasoning has resulted in the development of a theory-based preventive home visitation programme for potentially frail community-dwelling older people implemented by general practices, called [G]OLD ('Getting OLD the healthy way'). By applying components of the Chronic Care Model $^{21}$ and the Guided Care model, ${ }^{22}$ the programme consists of a comprehensive geriatric assessment (CGA) of older people's health and well-being problems or needs, a tailored care and treatment plan, multidis- 
ciplinary care management, and targeted intervention and follow-up. Although a pilot study revealed that the [G]OLD home visitation programme was judged positively by the care professionals involved, ${ }^{23}$ the effects on older people's health-related quality of life and disability have not been studied yet. Therefore, this study aims to investigate the effectiveness of the early identification of health and well-being problems among potentially frail community-dwelling older people (aged $\geq 75$ years) by means of a comprehensive geriatric assessment and follow-up within the chain of care. We hypothesised that a nurse-led preventive home visitation programme within general practices will lead to improved health-related quality of life and reduced disability compared to general practices who provide usual care (i.e., reactive care) to potentially frail community-dwelling older people.

\section{METHODS}

\section{Study design and setting}

A longitudinal, cluster quasi-experimental controlled trial was performed among 24 general practices in three regions in the south of the Netherlands. To prevent contamination between intervention and control participants, thirteen general practices ( 7 from the region 'Parkstad', 6 from the region 'Maastricht-Heuvelland) were allocated to the intervention group and implemented the [G]OLD preventive home visitation programme, whereas eleven general practices ( 2 from the region 'Parkstad', 9 from the region 'Midden-Limburg') were allocated to the control group and provided usual care (i.e., reactive care instead of proactive care) to older people. Further details regarding the study design are published elsewhere. ${ }^{24}$

According to the Medical Research Involving Human Subjects Act, no formal ethical approval was needed for this study. Nevertheless, the Medical Ethical Committee (MEC) of Maastricht University Medical Centre (MUMC+) granted their approval for this study and related documents.

\section{Participants}

Except for general practices who participated in our pilot study $(n=21)$, all remaining 188 general practices in the three regions were approached for participation. Those who had already embedded a systematic policy for proactive care for potentially frail community-dwelling older people into their daily care delivery were excluded $(n=12)$. The availability of a PN with time to perform the home visits and follow-up of older people over time was a prerequisite for inclusion in the intervention group. Participa- 
tion in the control group necessitated no involvement in any form of proactive screening and follow-up of potentially frail community-dwelling older people for up to two years. Following these requirements, 24 general practices were included, of which 13 general practices consented to participate in the intervention group and 11 general practices participated in the control group. General practices were matched on the number of GPs and the presence of other primary care professionals within general practice besides the GP and PN. Team structure, including proximity of various primary care professionals in the same building, is an essential element of successful interprofessional collaboration in primary care, ${ }^{25}$ and therefore important for the organisation and delivery of care to older people. Other characteristics, namely type of practice (i.e., solo, duo, group), size of the patient population $\geq 75$ years per GP, to what extent the practice is currently actively involved in care for older people (measured on a 5point scale ranging from 'not at all' to 'very much'), whether the practice has spent more attention to care for older people in the past two years than before (measured on a 4-point scale ranging from 'totally disagree' to 'totally agree'), and the extent to which the practice has a positive attitude towards initiatives in care for older people (measured on a 5-point scale ranging from 'not at all' to 'very much') were determined using a questionnaire distributed among general practices after recruitment to compare the clusters.

All community-dwelling older people aged 75 years and older were eligible to participate. General practices used the age criterion to select eligible older people from their GP Information System. Older people not living independently, those on a waiting list for admission to a nursing home or home for older people, those under close medical supervision (chemotherapy, chronic haemodialysis or other therapies posing a high burden on the older person), and the terminally ill were excluded. In the intervention group, the maximum number of older people that the PN would be able to visit within approximately one year time, was used as an additional criterion to randomly select older people from all those eligible to participate. Older people were approached for participation by means of an information letter and informed consent form. After two to five weeks, non-responders to the first mailing received either a telephone reminder (intervention group) or a postal reminder (control group).

\section{Intervention}

The 13 general practices in the intervention group were offered a comprehensive approach for planning and executing care for potentially frail older people based on principles of the Chronic Care Model (CCM) ${ }^{21}$ and the Guided Care Model ${ }^{22}$ (for details on the development of the intervention, $s^{23}{ }^{23}$. These models have proven successful in optimising primary care and achieving better health outcomes among people with 
chronic diseases and complex care needs. ${ }^{26,27}$ General practices redesigned their care delivery from offering care upon request towards proactive care by means of the early detection of the health and well-being needs of older people. The PN, in collaboration with the GP, was the key actor responsible for conducting the [G]OLD home visitation programme.

The protocol of the [G]OLD home visitation programme (see Figure 7.1) starts with the PN inviting the older person for a home visit by phone. After the appointment is made, the PN prepares the visit by extracting relevant information from the GP Information System (e.g., medication overview, medical history). Then, the PN visits the older person at home and performs a comprehensive geriatric assessment of physical, psychological, mental and social functioning, lifestyle and medication use by means of the [G]OLD-instrument. ${ }^{23}$ Based on cut-off points as presented in the [G]OLDinstrument, a list of potential problems or needs is generated which is discussed with the GP. If necessary, the PN performs a more elaborate assessment using the [G]OLDinstrument part two to obtain insight into the presence or absence of cognitive disorders, depression, and personality disorders. The results of the assessment, together with the older person's needs and wishes, determine whether follow-up actions are needed for detected problems or risk situations. In that case, the PN and/or GP formulate a care and treatment plan, which is discussed with the older person. Subsequently, the PN executes the care and treatment plan by referring the older person to individually appropriate health and/or well-being services in the neighbourhood. The PN and/or GP participate in multidisciplinary meetings with other care professionals, (e.g., physiotherapist, occupational therapist) to attune care to older people's needs. Finally, the PN evaluates progress, coordinates care, and monitors follow-up of the older person over time (e.g., by phone, home visits, or a re-assessment with the [G]OLDinstrument). The number of follow-up contacts is not fixed, but depends on the type of problems or complaints and the older person's wishes.

PNs randomly approached older people who consented to participate in this study and the home visits were scheduled consecutively between July 2010 and September 2011. Before the start of the intervention period, all PNs participated in two training sessions and in between they performed five home visits among randomly chosen community-dwelling older people ( $\geq 75$ years) (try-out phase). During the intervention period, PNs received coaching on the job to provide them with the necessary knowledge and skills to carry out the different steps of the [G]OLD home visitation programme. 


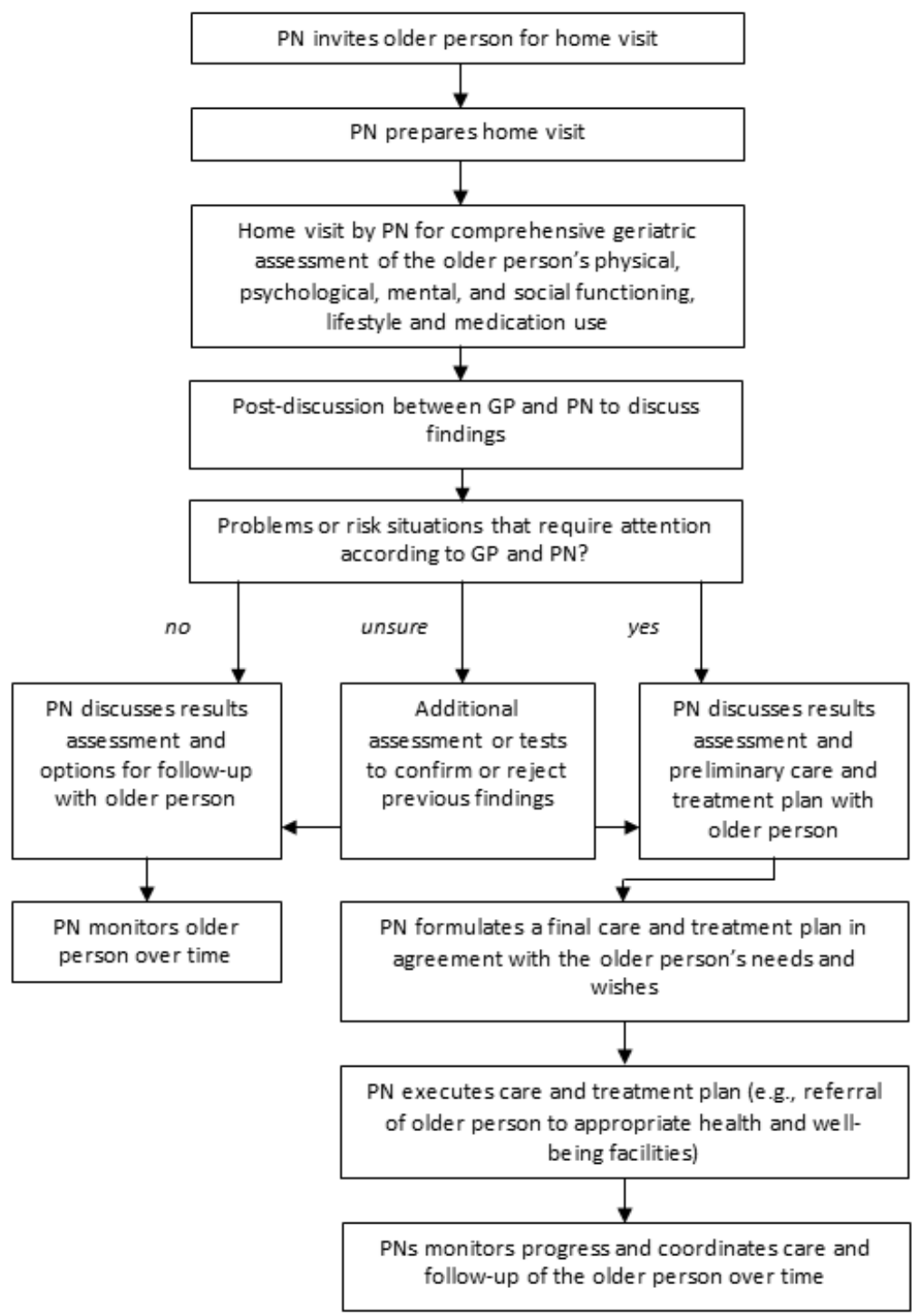

Figure 7.1 Overview [G]OLD home visitation programme (intervention protocol)

\section{Measurements}

Primary outcome measures were health-related quality of life measured by the RAND$36^{28,29}$ and disability measured by the Groningen Activity Restriction Scale (GARS). ${ }^{30}$ The RAND-36 consists of 8 dimensions: physical functioning (10 items), social functioning ( 2 items), role limitations due to physical health problems (4 items), role limitations 
due to emotional health problems ( 3 items), emotional well-being ( 5 items), energy/fatigue (4 items), pain ( 2 items), general health perceptions (5 items), and change in perceived health (1 item). The GARS is an 18-item questionnaire comprising two subscales: activities of daily living (ADL; 11 items) and instrumental activities of daily living (IADL; 8 items). Scores on the total scale range from 18 to 72 with higher scores indicating higher levels of disability. Both the RAND-36 and the GARS are valid and reliable instruments to measure the primary outcomes and suitable for self-completion by older people. ${ }^{31,32}$ In addition, we included attitude towards ageing from the Philadelphia Geriatric Centre Morale Scale ${ }^{33}$ as a secondary outcome measure. This 5-item scale covers an older person's perception of the changes taking place in his/her life. Though concrete evidence of its psychometric properties among Dutch older people is lacking, the scale is specifically designed for application in older subjects ${ }^{34}$ and its internal consistency at baseline was reasonable $(\alpha=0.70)$. All outcome measures were included in a questionnaire sent to older people at baseline and after 6-months, 12-months, and 18months follow-up.

\section{Sample size calculation}

The sample size calculation was based on the primary outcome health-related quality of life. To demonstrate a clinically relevant treatment difference of 5.0 on the transformed subscale 'general health perception', given $\mathrm{SD}=21.2$, an $\alpha$ of 0.05 and a power of 0.80 , the minimally required number of participants was 282 per study group. Calculations taking into account the interdependency of the measurements within a cluster (intraclass correlation coefficient of 0.01 ) and a cluster effect of 1.52 , resulted in a required sample size of 960 older people ( $n=480$ per study group). Accounting for an expected response rate of $50 \%$ and $30 \%$ attrition, at least 1,371 community-dwelling older people per study group had to be approached for participation. Unequal cluster sizes were compensated for by sampling $25 \%$ more clusters (i.e., general practices). ${ }^{35}$

\section{Statistical analyses}

Descriptive statistics were computed to describe the sample characteristics. We included participants who completed at least one out of four questionnaires. Missing data at item-level were handled following the instructions of the developers of the questionnaires. If no guidelines were available, we allowed a maximum of $25 \%$ missing values and substituted missings by the individual average score on the remaining items of a scale. Hereafter, scale scores were computed for RAND-36 (range: 0-100), GARS (range: 18-72), and attitude towards ageing (range: 0-5). Non-normally distributed outcome measures (subscales physical functioning, role limitations caused by physical 
and emotional health problems, social functioning, pain, total score GARS, and subscales $A D L$ and $I A D L$ ) were categorised, as various transformations (i.e., log, square root, reciprocal) did not result in normal distributions. Data were analysed using generalised mixed effects models to account for nesting of participants within general practices and for correlated data due to repeated measures.

At the general practice level, baseline differences between the intervention and control group were explored using ordinal logistic regression (for ordinal variables), and independent-samples t-tests (for continuous variables). Baseline differences between elderly participants in the intervention and control group were examined with linear mixed regression analyses (for continuous variables), binary logistic mixed regression analyses (for binary variables), and ordinal logistic mixed regression analyses (for ordinal variables) with 'group' as fixed effect and 'practice' as random effect. In case the variance of the random intercept at 'practice'-level was estimated to be zero or was non-significant (using a one-tailed test), 'practice' was removed as a level and baseline characteristics were compared using chi-square tests (nominal variables), ordinal logistic regression (ordinal variables), and independent-samples t-tests (continuous variables). Selective drop-out was assessed by binary logistic mixed regression with drop-out per measurement as the dependent variable (questionnaire returned yes/no) and 'group', baseline characteristics of participants (age, sex, educational level, living status, degree of urbanisation), baseline score of the outcome measures, and baseline characteristics of general practices (type of practice, size of the patient population $\geq 75$ years per GP, past and current involvement in care for older people, and attitude towards initiatives in care for older people) as fixed effects.

Primary and secondary outcomes were analysed according to the intention-totreat principle, in that all available data were analysed. Random intercept linear mixed regression analyses with three levels (repeated measures nested within subjects nested within general practices) were done for continuous outcomes and ordinal logistic mixed regression analyses for ordinal outcomes, including the interaction group by time to represent the intervention effect and 'subjects' and 'practice' as random effects. Baseline characteristics of participants, baseline characteristics of general practices, outcome measures showing significant differences between the study groups at baseline, and significant predictors of drop-out were included as covariates in each model, also as interaction terms with time. Non-significant random effects (using a one-tailed test) were removed from the model. A top-down approach was used for model selection starting with the most extensive model. Firstly, non-significant covariate by time interactions were removed from the model. Secondly, non-significant covariates were removed, resulting in a final model only containing significant main and interaction effects of covariates and also the group by time interaction effect. 
We used IBM SPSS Statistics for Windows, version 21.0, for all statistical analyses. A significance level of $\mathrm{P}<0.10$ was applied as a conservative approach in the drop-out analyses, as well as when testing baseline differences between study groups and when excluding random effects and main and interaction effects of covariates from the analysis model. ${ }^{36}$ In all other cases, a significance level of $\mathrm{P}<0.05$ was used.

\section{RESULTS}

\section{Sample characteristics}

Characteristics of the 24 participating general practices are summarised in Table 7.1. The number of GPs working in the general practice was equally distributed between the study groups. In intervention practices, somewhat more other care professionals besides the GP and PN were present in the general practice. With respect to the remaining cluster characteristics, intervention practices were significantly more involved in care for older people at recruitment and in the past compared to control practices. Furthermore, intervention practices had a significantly more positive attitude towards care for older people than control practices.

Table 7.1 Characteristics of general practices $(n=24)$

\begin{tabular}{|c|c|c|}
\hline & $\begin{array}{l}\text { Intervention } \\
\text { practices }(n=13)\end{array}$ & $\begin{array}{l}\text { Control } \\
\text { practices }(n=11)\end{array}$ \\
\hline \multicolumn{3}{|l|}{ Number of GPs ${ }^{a}$} \\
\hline One & $4(30.8)$ & $4(36.4)$ \\
\hline Two & $6(46.1)$ & $5(45.4)$ \\
\hline Three & $1(7.7)$ & $0(0)$ \\
\hline Four & $2(15.4)$ & $2(18.2)$ \\
\hline $\begin{array}{l}\text { Other care professionals besides GP and PN that are present } \\
\text { in the practice }\end{array}$ & $11(84.6)$ & $7(63.6)$ \\
\hline \multicolumn{3}{|l|}{ Type of practice $^{a}$} \\
\hline Solo practice & $6(46.1)$ & $5(45.4)$ \\
\hline Duo practice & $4(30.8)$ & $4(36.4)$ \\
\hline Group practice & $3(23.1)$ & $2(18.2)$ \\
\hline Size of the patient population aged $\geq 75$ years per GP ${ }^{b}$ & $151.5(63.07)$ & $161.02(82.83)$ \\
\hline Current involvement in care for older people ${ }^{b}$ & $3.69 * *(1.03)$ & $3.00 * *(0.89)$ \\
\hline Past involvement in care for older people ${ }^{b}$ & $3.04 *(0.66)$ & $1.91 *(0.83)$ \\
\hline Positive attitude towards initiatives in care for older people ${ }^{b}$ & $4.46^{*}(0.52)$ & $3.64 *(0.81)$ \\
\hline
\end{tabular}


The flow of participants through each stage of the study is shown in Figure 7.2. The total patient population aged $\geq 75$ years in 24 general practices was 6,850 individuals. They were assessed for eligibility, resulting in 4,588 community-dwelling older people (67.0\%) who fulfilled the inclusion criteria and were approached for participation. Written informed consent was obtained from 1,737 older people (37.9\%). At recruitment, non-responders were significantly older (mean age 81.42 vs 80.87 years; $P=0.00$ ) and more likely to be female (63.1\% vs $56.0 \%$; $\mathrm{P}=0.00$ ) than responders. Moreover, non-responders were significantly more often labelled as potentially frail by their GP than responders $(47.3 \%$ vs $40.5 \% ; \mathrm{P}=0.00$ ). In the intervention group, baseline questionnaires were sent to older people after the home visit was scheduled and PNs visited participants stepwise between July 2010 and September 2011. In this period, the participants in the control group received the baseline questionnaire also stepwise (grouped per cluster). Due to this stepwise approach, 210 older people dropped out before baseline and eventually 1,527 older people were included in this study. The [G]OLD home visitation programme was received by 571 participants $(97.4 \%$ of those enrolled in the intervention group). For 15 older people a home visit was initially scheduled by the PN, but did not take place and could not be rescheduled during the intervention period due to the older person's circumstances (e.g., illness, admission, death). 


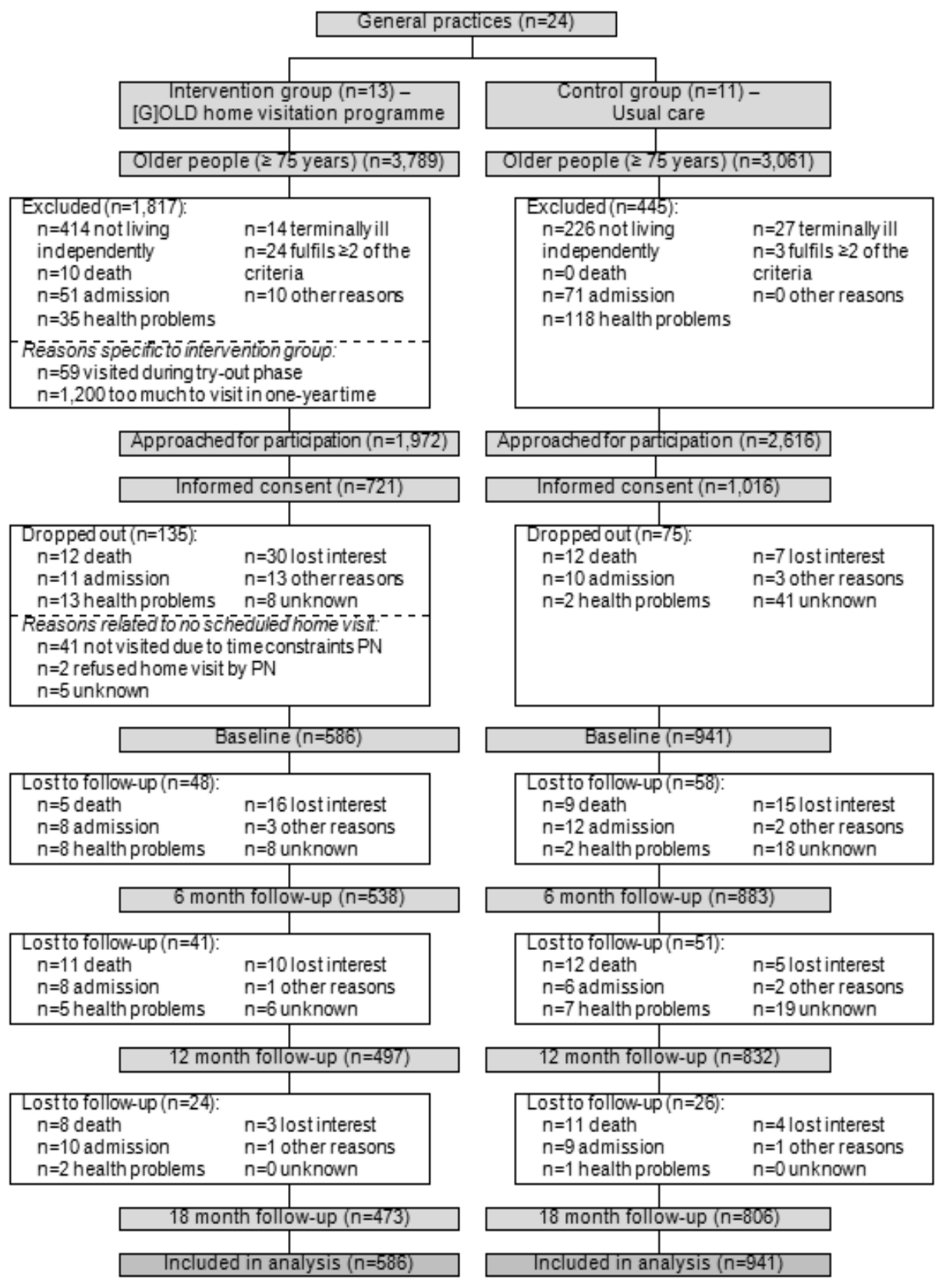

Figure 7.2 Flowchart of participants 
Overall, the mean age of participants was 81.07 years $(S D=4.05), 56.0 \%$ were female, 47.2\% lived alone; the majority had completed lower professional education (42.4\%) and lived in a rural area (40.2\%) (Table 7.2). Baseline differences between participants in the intervention group and control group were significant for living status, level of education, and all primary outcome measures and the secondary outcome measure. Intervention group participants more often lived alone $(\mathrm{P}=0.07)$ and had a lower educational level than those in the control group $(P=0.07)$. In addition, intervention group participants reported better physical functioning $(P=0.00)$, less role limitations due to physical and emotional problems $(P=0.00$ and $P=0.01)$, worse social functioning $(P=0.08)$, worse emotional well-being $(P=0.04)$, lower levels of energy and more fatigue $(P=0.00)$, less pain $(P=0.00)$, a lower general health perception $(P=0.00)$, a less positive change in perceived health $(P=0.05)$, higher levels of disability (total score and in both $A D L$ and IADL scores) $(P=0.00, P=0.01$, and $P=0.00)$, and a more negative attitude towards ageing $(P=0.01)$ compared to control group participants.

\section{Drop-out analyses}

Of the 1,527 participants, $1,428(93.5 \%)$ returned the questionnaire at baseline, 1,339 (87.7\%) at 6 months follow-up, 1,287 (84.3\%) at 12 months follow-up, and 1,160 $(76.0 \%)$ at 18 months follow-up. Type of practice, size of the patient population $\geq 75$ years per GP, current involvement in care for older people, and attitude towards initiatives in care for older people were significant predictors of retention at baseline. At 6 months follow-up, sex, age, IADL functioning at baseline, attitude towards ageing at baseline, type of practice, and current involvement in care for older people were significant predictors of retention. At 12 months follow-up, age, social functioning at baseline, change in perceived health at baseline, ADL functioning at baseline, IADL functioning at baseline, and past involvement in care for older people were significant predictors of retention. Finally, age, degree of urbanisation, level of education, social functioning at baseline, ADL functioning at baseline, attitude towards ageing at baseline, type of practice, and past involvement in care for older people, were significant predictors of retention at 18 months follow-up. 
Table 7.2 Baseline characteristics of participants $(n=1,527)$

\begin{tabular}{|c|c|c|c|c|c|c|}
\hline & $\mathrm{n}$ & $\begin{array}{l}\text { Overall } \\
(n=1,527)\end{array}$ & $\mathrm{n}$ & $\begin{array}{l}\text { Intervention group } \\
\qquad(n=586)\end{array}$ & $\mathrm{n}$ & $\begin{array}{l}\text { Control group } \\
\qquad(\mathrm{n}=941)\end{array}$ \\
\hline Age (years) ${ }^{a}$ & 1,428 & $81.07(4.05)$ & 547 & $81.15(4.22)$ & 881 & 81.03 (3.95) \\
\hline Female sex ${ }^{b}$ & 1,527 & $855(56.0)$ & 586 & $332(56.7)$ & 941 & $523(55.6)$ \\
\hline Living alone $^{\mathrm{b}}$ & 1,419 & $670(47.2)$ & 542 & $279 * *(51.5)$ & 877 & $391 * *(44.6)$ \\
\hline Level of education & 1,415 & & 540 & $* *$ & 875 & $* *$ \\
\hline No or only primary education ${ }^{b}$ & & $417(29.5)$ & & $176(32.6)$ & & $241(27.5)$ \\
\hline Lower professional education ${ }^{b}$ & & $600(42.4)$ & & $235(43.5)$ & & 365 (41.7) \\
\hline Middle professional education ${ }^{\mathrm{b}}$ & & 209 (14.8) & & $72(13.3)$ & & $137(15.7)$ \\
\hline Higher professional education ${ }^{b}$ & & $189(13.3)$ & & $57(10.6)$ & & $132(15.1)$ \\
\hline Degree of urbanisation & 1,527 & & 586 & & 941 & \\
\hline Rural $^{\text {b }}$ & & $614(40.2)$ & & $230(39.2)$ & & $384(40.8)$ \\
\hline Predominantly rural ${ }^{\mathrm{b}}$ & & $192(12.6)$ & & $192(32.8)$ & & 0 \\
\hline Intermediate rural-urban ${ }^{\mathrm{b}}$ & & $416(27.2)$ & & 0 & & $416(44.2)$ \\
\hline Urban $^{\mathrm{b}}$ & & $305(20.0)$ & & $164(28.0)$ & & $141(15.0)$ \\
\hline \multicolumn{7}{|l|}{ Health-related quality of life } \\
\hline Physical functioning ${ }^{a}$ & 1,355 & $39.98(28.52)$ & 517 & $44.31 *(28.55)$ & 838 & $37.31 *(28.19)$ \\
\hline $\begin{array}{l}\text { Role limitations caused by physical } \\
\text { health problems }{ }^{\text {a }}\end{array}$ & 1,329 & $44.65(43.45)$ & 504 & $49.65 *(44.05)$ & 825 & $41.60 *(42.83)$ \\
\hline $\begin{array}{l}\text { Role limitations caused by emotional } \\
\text { health problems }{ }^{\text {a }}\end{array}$ & 1,321 & $27.49(39.91)$ & 497 & $32.63 *(42.24)$ & 824 & $24.39 *(38.14)$ \\
\hline Social functioning ${ }^{a}$ & 1,423 & $75.34(24.82)$ & 545 & $72.75 * *(25.62)$ & 878 & $76.95^{* *}(24.19)$ \\
\hline Emotional well-being ${ }^{a}$ & 1,358 & $75.10(17.13)$ & 515 & $73.27 *(18.17)$ & 843 & $76.22 *(16.38)$ \\
\hline Energy/fatigue $^{a}$ & 1,380 & 60.99 (19.97) & 527 & $57.86 *(20.66)$ & 853 & $62.93 *(19.29)$ \\
\hline Pain ${ }^{a}$ & 1,413 & $69.18(25.60)$ & 544 & $64.74 *(26.03)$ & 869 & $71.95 *(24.94)$ \\
\hline General health perceptions $^{a}$ & 1,368 & $57.63(17.41)$ & 517 & $55.18 *(17.41)$ & 851 & $59.11 *(17.25)$ \\
\hline Change in perceived health ${ }^{a}$ & 1,398 & $43.44(17.98)$ & 537 & $42.22 *(18.10)$ & 861 & $44.19 *(17.87)$ \\
\hline \multicolumn{7}{|l|}{ Disability } \\
\hline Total score ${ }^{a}$ & 1,363 & $28.46(10.57)$ & 521 & $29.63 *(10.96)$ & 842 & $27.74 *(10.27)$ \\
\hline ADL scale ${ }^{a}$ & 1,384 & $15.45(5.47)$ & 533 & $15.87 *(5.76)$ & 851 & $15.19 *(5.27)$ \\
\hline IADL scale ${ }^{a}$ & 1,353 & $13.01(5.71)$ & 524 & $13.73 *(5.82)$ & 829 & $12.56 *(5.60)$ \\
\hline Attitude towards ageing ${ }^{a}$ & 1,335 & $2.31(0.90)$ & 504 & $2.23 *(0.91)$ & 831 & $2.36 *(0.89)$ \\
\hline \multicolumn{7}{|l|}{ Healthcare utilisation } \\
\hline No. of contacts GP past 3 months ${ }^{a}$ & 1,386 & $1.49(1.73)$ & 523 & $1.54(1.80)$ & 863 & $1.46(1.69)$ \\
\hline $\begin{array}{l}\text { No. of contacts medical specialist in } \\
\text { hospital past } 3 \text { months }{ }^{\text {a }}\end{array}$ & 1,390 & $1.19(2.01)$ & 526 & $1.20(2.27)$ & 864 & $1.19(1.83)$ \\
\hline $\begin{array}{l}\text { No. of days in hospital past } 3 \\
\text { months }{ }^{\text {a }}\end{array}$ & 1,420 & $0.51(3.44)$ & 542 & $0.76(4.84)$ & 878 & $0.36(2.15)$ \\
\hline
\end{tabular}


* significant at $\mathrm{p}<0.05 ; * *$ significant at $\mathrm{p}<0.10$.

${ }^{a}$ mean (SD); ${ }^{b}$ number (percentage).

Note: Physical functioning (range: 0-100): a higher score indicates better physical functioning; Role limitations (due to physical or emotional problems) (both range: 0-100): a higher score indicates less role limitations; Social functioning (range: 0-100): a higher score indicates better social functioning; Emotional well-being (range: 0-100): a higher score indicates better emotional well-being; Energy/fatigue (range: 0100): a higher score indicates higher levels of energy and less fatigue; Pain (range: 0-100): a higher score indicates less pain; General health perceptions (range: 0-100): a higher score indicates a better general health perception; Change in perceived health (range: 0-100): a higher score indicates a positive change in perceived health; Disability (range total scale: 18-72; range Activities of Daily Living (ADL) scale: 11-44; range instrumental ADL (IADL) scale: 7-28): a higher score indicates more disability; Attitude towards own ageing (range: 0-5): a higher score indicates a more positive attitude towards ageing.

\section{Effect on primary and secondary outcomes}

Table 7.3 presents the results of the multilevel analyses for both primary and secondary outcomes. The intervention group showed significantly less role limitations due to physical problems at 6 months follow-up $(P=0.045)$ and 18 months follow-up $(P=0.044)$ than the control group. Furthermore, significant differences between the intervention group and control group were found for social functioning at all time points ( $P=0.001$, $\mathrm{P}=0.000$ and $\mathrm{P}=0.029$, respectively) and for attitude towards ageing at 6 months follow-up $(P=0.038)$, whilst these effects were all in favour of the control group.

\section{DISCUSSION}

This study shows that a nurse-delivered preventive home visitation programme for potentially frail community-dwelling older people, consisting of a comprehensive geriatric assessment, a tailored care and treatment plan, multidisciplinary care management, and targeted intervention and follow-up had a small but statistically significant effect on role limitations due to physical problems at 6 months and 18 months followup compared to usual care (i.e., reactive care) delivered by general practices. We found no statistically significant effects of the [G]OLD home visitation programme on all other primary and secondary outcome measures.

These findings are in line with other recent studies in the Netherlands in which complex home visiting interventions for community-dwelling older people were implemented in general practices. ${ }^{37,38}$ Metzelthin and colleagues ${ }^{37}$ found no evidence for the effectiveness of a proactive approach consisting of multidimensional assessment, interdisciplinary care, tailored treatment plan, and regular evaluation and follow-up among frail older people ( $\geq 70$ years) on disability. Blom and co-workers ${ }^{38}$ developed a proactive, integrated intervention comprising a multidimensional assessment and a 
tailored treatment plan for older people ( $\geq 75$ years) with problems in multiple domains and found no effects on daily functioning and quality of life. In contrast to these findings, a Dutch intervention targeted at vulnerable community-dwelling older people ( $\geq 70$ years) with known problems, comprising a multidimensional assessment, individualised treatment plan, and geriatric evaluation and management, was able to show improvement in functional abilities and mental well-being. ${ }^{39}$ Also internationally, the effects of complex interventions in the primary care setting are inconsistent. For example, whereas a French study found that integrated primary care with intensive case management among community-dwelling, very frail older persons ( $\geq 65$ years) reduced unplanned hospitalisations and improved some health parameters, ${ }^{40}$ a Canadian study found no effect of a preventive primary care outreach intervention for older people ( $\geq 75$ years) at risk of functional decline on functional status, self-rated health, and mortality. $^{41}$

All interventions mentioned above include elements that have shown to be conducive to positive outcomes in older people, such as a comprehensive geriatric assessment ${ }^{13}$, yet the results remain inconsistent. Therefore, the description of a clear theory guiding the intervention is advocated. ${ }^{11}$ The [G]OLD home visitation programme contains elements derived from evidence-based care models (i.e., the Chronic Care Model and the Guided Care model), though not all principles of these models were incorporated in the intervention protocol. Although successful results have been obtained following the implementation of some of the elements of the Chronic Care Model, the best outcomes are obtained when changes are made across multiple elements of the Chronic Care Model. ${ }^{27}$ However, we cannot assert that otherwise successful results would have been obtained, since intervention content alone is insufficient to elicit favourable intervention effects. Several other possible explanations exist, besides general failure of the [G]OLD home visitation programme, for the lack of effects at the individual level. Firstly, as previous studies already suggested, ${ }^{37,41}$ due to a well-established primary healthcare system in the Netherlands, easy access to the GP, and the introduction of various chronic disease management programmes in general practice, ${ }^{42}$ older people may already have received appropriate health and well-being services as part of usual care, which restricts the ability of a primary care intervention to produce additional beneficial outcomes at the patient level. Consequently, it might be worthwhile to reconsider the needs of care professionals and to disclose which elements of existing intervention programmes are most effective in tackling the complex care demands of the emerging population of potentially frail older people before introducing any new complex intervention programmes. 


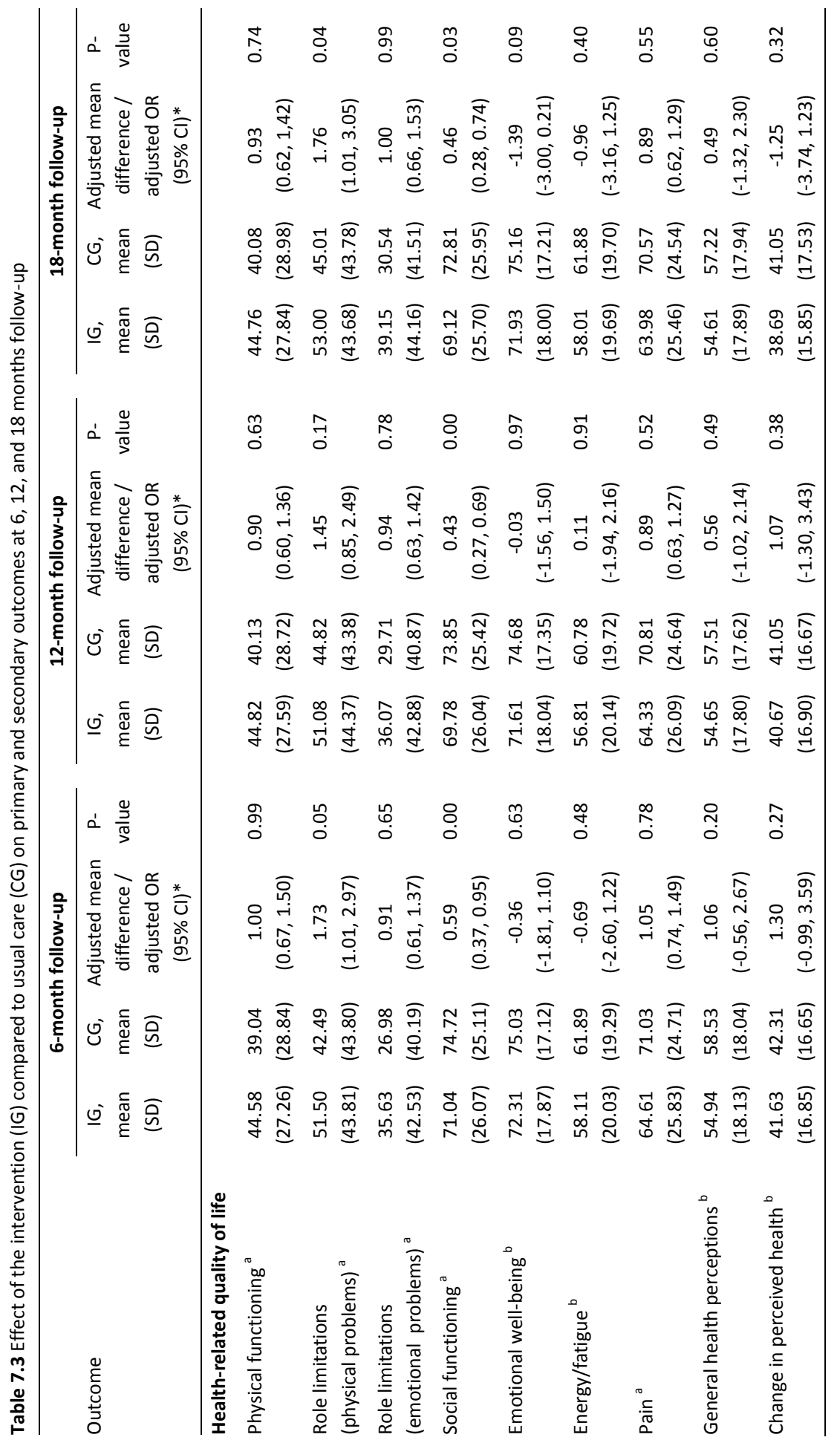




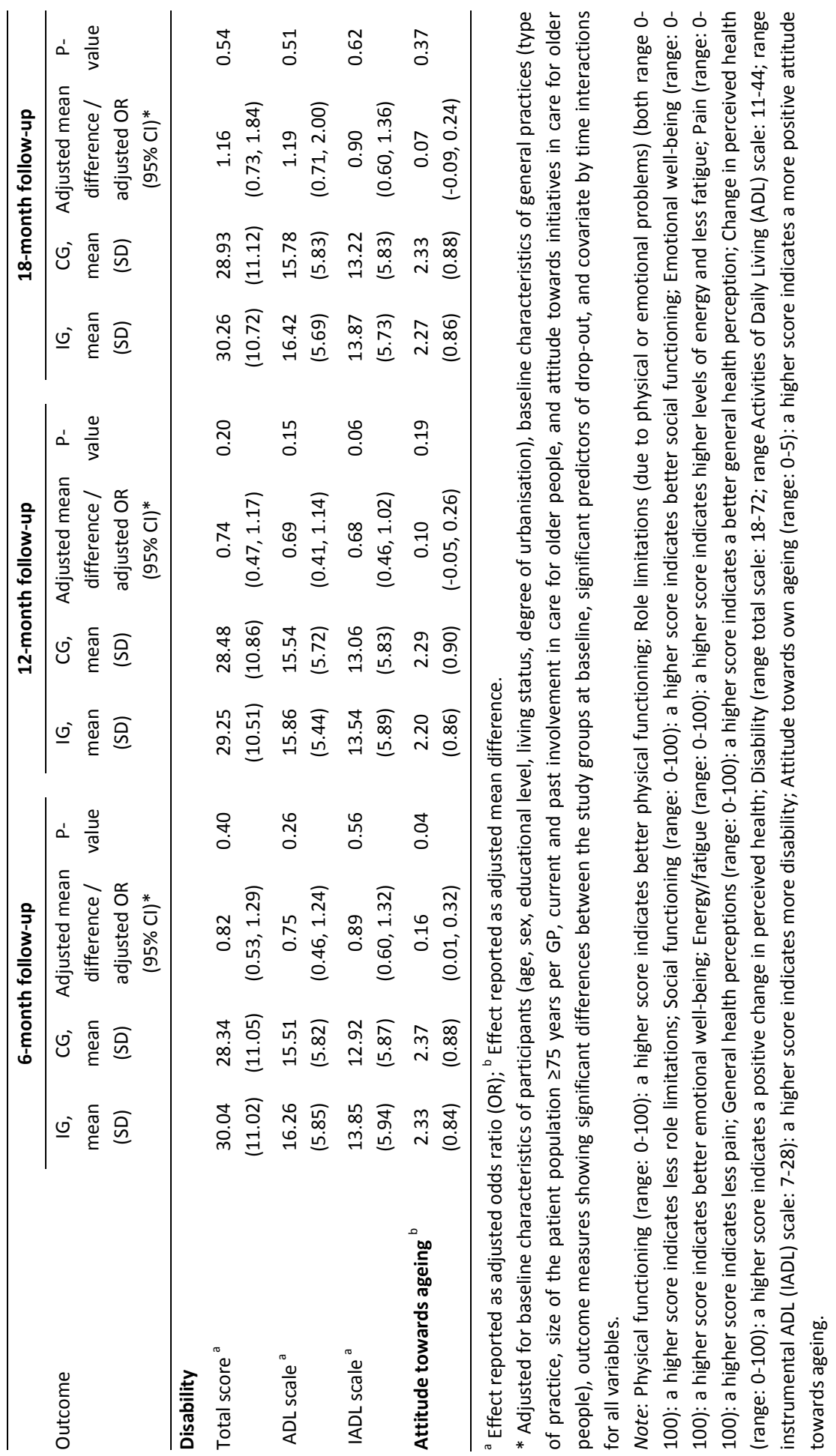


Secondly, our process evaluation suggests that implementation fidelity of the [G]OLD home visitation programme was relatively low, as the intervention was delivered according to protocol to only $38.0 \%$ of the 721 older people who gave informed consent. ${ }^{43}$ For example, there was a large variety in the percentage of formulated care and treatment plans per PN (4.0\% to $95.2 \%)$. Also, only approximately one out of four detected problems were included in a care plan, mostly because it was an existing problem and/or action had already been undertaken or the older person did not want any follow-up actions. ${ }^{44}$ The most frequently mentioned causes for inadequate implementation of the intervention as a whole were time constraints of the PN and few newly detected health and/or well-being problems. ${ }^{44}$ Furthermore, the complexity of the [G]OLD home visitation programme in terms of actions that needed to be undertaken to redesign care delivery from reactive to proactive care may also have added to the intervention not being fully implemented as planned. This underlines the importance of measuring fidelity and the moderating factors influencing fidelity of multicomponent interventions implemented in complex settings. ${ }^{45}$ Thirdly, the target population may have influenced the results obtained. Potentially frail community-dwelling older people were included in this study, meaning that all older people aged 75 years and older poses some degree of risk for frailty now or at a certain point in the near future. This is inherent to a public health perspective towards prevention among older people in which a population-based screening approach ultimately results in controlling the causes of frailty instead of targeted screening and follow-up among individuals at high risk. ${ }^{46}$ As a result, also the relatively healthy older people not labelled as potentially frail by their GP (i.e., 59.5\%) were included in the study sample and this may have diluted the intervention effect. Moreover, a multidimensional assessment among purposefully selected high-risk individuals results in more detected health and well-being problems, ${ }^{47}$ and consequently these older people may be more likely to benefit from the [G]OLD home visitation programme. Yet one of the largest trials in this area found no evidence that older people are more likely to benefit from targeted assessment compared to a population-based assessment. ${ }^{48}$ Some studies have even found evidence for the effectiveness of preventive home visits among well-functioning older people ${ }^{49}$ or those at low-risk for death. ${ }^{13}$ Briefly stated, a tailored approach seems most appropriate, whereby older people at low risk of disability receive multidimensional preventive interventions (e.g., lifestyle advice), whereas those at high risk receive multidimensional interventions targeting specific problems. ${ }^{50}$ We tried to incorporate this tailored approach in the [G]OLD home visitation programme, but primary prevention among low risk individuals probably failed because older people without problems or a specific request for help often did not want to undertake any actions. ${ }^{43,44}$ It seems that older people are not used to the proactive approach of their general practice or they consider certain health conditions (e.g., memory impairment) 
as inevitable aspects of ageing for which there is no need to seek healthcare. ${ }^{51}$ Older people's awareness should be stimulated that the GP and PN deliver proactive, preventive services for a variety of health and well-being problems, also outside the medical domain.

The strengths of this study are the allocation of clusters (i.e., general practices) to either the intervention group or control group to prevent contamination bias. Compared to other studies, we were able to include a larger sample of older people and we had sufficient power to detect effects on the outcome measures. Predominantly validated questionnaires were used to measure the outcomes and we achieved a relatively high response on the different measurements $(93.5 \%$ at baseline and $76.0 \%$ at 18 months follow-up). However, there are also several limitations. Firstly, the quasiexperimental design restricts the ability to draw causal inferences from the home visitation programme with respect to the outcome measures. A cluster randomised controlled trial would have provided better scientific evidence. ${ }^{52}$ However, randomisation (i.e., to balance known and unknown prognostic factors between study groups) is not always feasible, because home visitation programmes often comprise a complex mix of uncontrollable prognostic factors. ${ }^{53}$ Secondly, due to the recruitment of general practices from different regions in respectively the intervention group and control group, intervention practices and control practices showed significant differences in some socio-demographic characteristics and all outcome measures at baseline. Although we matched general practices as much as possible on relevant characteristics and baseline differences were corrected for in the multilevel analyses, this may still have had a negative influence on the intervention effect. Furthermore, at baseline, intervention practices showed greater past and current involvement in care for older people and they had a more positive attitude towards care for older people than control practices. As a result, older people within intervention practices might already have received adequate care and well-being services as part of usual care before the start of this study, and thus, the intervention did not provide any additional beneficial effects. Thirdly, selection bias could have influenced the results. Due to the informed consent procedure, only $37.9 \%$ of the eligible older people agreed to participate and these were the young-old and they were less likely to be classified as frail by their GP. This suggests that the more functionally independent older people participated and this can be confirmed by a non-response study among older people in the Netherlands which found that non-participants showed better ADL and IADL functioning than participants. ${ }^{54}$ Furthermore, at all follow-up measurements retention was selective for younger participants and those who reported better ADL or IADL functioning, which may have diluted any preventive effects on the self-reported outcome measures. Fourthly, as the intervention had no fixed endpoint and continued during the follow-up period of the trial, the duration of follow-up was probably too short to find significant effects of the 
intervention on relatively distal outcome measures such as health-related quality of life and disability. Finally, the effect study started simultaneously with the implementation of the intervention in general practices. As our process evaluation suggests, it takes some time before the intervention protocol is fully embedded in general practices, professionals are used to the new way of working, and all elements are performed as planned. Thus, the intervention was evaluated while it was not yet delivered in the best possible manner.

\section{CONCLUSION}

This study adds to the available knowledge base on the effectiveness of home visitation programmes for community-dwelling older people. As the [G]OLD home visitation programme only had a small effect on role limitations due to physical problems compared to usual care, continuation of the intervention in its current form should be carefully reconsidered. Given some of the limitations of the current trial and the intervention being implemented with suboptimal fidelity, the findings might have been different under more optimal circumstances. The lack of clear effects of preventive home visits should not be used as a justification to discontinue these services among older people. However, before introducing any new complex intervention programmes, existing intervention models should be continuously improved and evaluated while implemented in the intended context to reveal elements that are conducive to positive outcomes in potentially frail community-dwelling older people. This seems especially justified given the increasing burden of the ageing population on healthcare systems. 


\section{REFERENCES}

1. Marengoni A, Angleman S, Melis R, Mangialasche F, Karp A, Garmen A, et al. Aging with multimorbidity: a systematic review of the literature. Ageing Res Rev 2011;10:430-439.

2. Fried TR, Tinetti ME, lannone L. Primary care clinicians' experiences with treatment decision making for older persons with multiple conditions. Arch Intern Med 2011;171:75-80.

3. Collard RM, Boter H, Schoevers RA, Oude Voshaar RC. Prevalence of frailty in community-dwelling older persons: a systematic review. J Am Geriatr Soc 2012;60:1487-1492.

4. Clegg A, Young J, Iliffe S, Olde Rikkert M, Rockwood K. Frailty in elderly people. Lancet 2013;381:752762.

5. Daniels R, Metzelthin S, Van Rossum E, De Witte L, Van den Heuvel W. Interventions to prevent disability in frail community-dwelling older persons: an overview. Eur J Ageing 2010;7:37-55.

6. Cameron ID, Fairhall N, Langron C, Lockwood K, Monaghan N, Aggar C, et al. A multifactorial interdisciplinary intervention reduces frailty in older people: randomized trial. BMC Med 2013;11:65.

7. Freer CB. Screening the elderly. Br Med J 1990;300:1447-1448.

8. Vass M, Avlund K, Hendriksen C, Philipson L, Riis P. Preventive home visits to older people in Denmark. Why, how, by whom, and when? Z Gerontol Geriatr 2007;40:209-216.

9. Elkan R, Kendrick D, Dewey M, Hewitt M, Robinson J, Blair M, et al. Effectiveness of home based support for older people: systematic review and meta-analysis. Br Med J 2001;323:719-725.

10. Huss A, Stuck AE, Rubenstein LZ, Egger M, Clough-Gorr KM. Multidimensional preventive home visit programs for community-dwelling older adults: a systematic review and meta-analysis of randomized controlled trials. J Gerontol A Biol Sci Med Sci 2008;63A:298-307.

11. Mayo-Wilson E, Grant S, Burton J, Parsons A, Underhill K, Montgomery P. Preventive home visits for mortality, morbidity, and institutionalization in older adults: a systematic review and meta-analysis. PLoS ONE 2014;9:e89257.

12. Van Haastregt JCM, Diederiks JPM, Van Rossum E, De Witte LP, Crebolder HFJM. Effects of preventive home visits to elderly people living in the community: systematic review. Br Med J 2000;320:754-758.

13. Stuck AE, Egger M, Hammer A, Minder CE, Beck JC. Home visits to prevent nursing home admission and functional decline in elderly people: systematic review and meta-regression analysis. J Am Med Assoc 2002;287:1022-1029.

14. Markle-Reid M, Browne G, Weir R, Gafni A, Roberts J, Henderson SR. The effectiveness and efficiency of home-based nursing health promotion for older people: a review of the literature. Med Care Res Rev 2006;63:531-569.

15. Van Hout HPJ, Jansen APD, Van Marwijk HWJ, Nijpel G. Prevention of adverse health trajectories in a vulnerable elderly population through nurse home visits: randomized controlled trial [ISRCTN05358495]. J Gerontol A Biol Sci Med Sci 2010;65:734-742.

16. Vass M, Avlund K, Siersma V, Hendriksen C. A feasible model for prevention of functional decline in older home-dwelling people-the GP role. A municipality-randomized intervention trial. Fam Pract 2009;26:56-64.

17. Lacas A, Rockwood K. Frailty in primary care: A review of its conceptualization and implications for practice. BMC Med 2012;10:4.

18. Boeckxstaens P, De Graaf P. Primary care and care for older persons: position paper of the European Forum for Primary Care. Qual Prim Care 2011;19:369-389.

19. Laurant M, Reeves D, Hermens R, Braspenning J, Grol R, Sibbald B. Substitution of doctors by nurses in primary care. Cochrane Database Syst Rev 2004;4:Art. No.: CD001271.

20. Van Weel C, Henk Schers H, Timmermans A. Health Care in The Netherlands. J Am Board Fam Med 2012;25:S12-17. 
21. Bodenheimer T, Wagner EH, Grumbach K. Improving primary care for patients with chronic illness. J Am Med Assoc 2002;288:1775-1779.

22. Boult C, Karm L, Groves C. Improving chronic care: the "Guided Care" model. Perm J 2008;12:50-54.

23. Stijnen MMN, Jansen MWJ, Vrijhoef HJM, Duimel-Peeters IGP. Development of a home visitation programme for the early detection of health problems in potentially frail community-dwelling older people by general practices. Eur J Ageing 2013;10:49-60.

24. Stijnen MMN, Duimel-Peeters IGP, Jansen MWJ, Vrijhoef HJM. Early detection of health problems in potentially frail community-dwelling older people by general practices - project [G]OLD: design of a longitudinal, quasi-experimental study. BMC Geriatr 2013;13:7.

25. Xyrichis A, Lowton K. What fosters or prevents interprofessional teamworking in primary and community care? A literature review. Int J Nurs Stud 2008;45:140-153.

26. Boyd CM, Reider L, Frey K, Scharfstein D, Leff B, Wolff J, et al. The effects of Guided Care on the perceived quality of health care for multi-morbid older persons: 18-month outcomes from a clusterrandomized controlled trial. J Gen Intern Med 2010;25:235-242.

27. Coleman K, Austin BT, Brach C, Wagner EH. Evidence on the chronic care model in the new millennium. Health Aff (Millwood) 2009;28:75-85.

28. Hays RD, Sherbourne CD, Mazel RM. The RAND 36-Item Health Survey 1.0. Health Econ 1993;2:217227.

29. Van der Zee KI, Sanderman R. Het meten van de algemene gezondheidstoestand met de RAND-36: een handleiding [Measurement of general health status with the RAND-36: a guideline]. Groningen: Rijksuniversiteit Groningen, Noordelijk Centrum voor Gezondheidsvraagstukken, 1993.

30. Kempen GIJM, Doeglas DM, Suurmeijer TPBM. Het meten van problemen met zelfredzaamheid op verzorgend en huishoudelijk gebied met de Groningen Activiteiten Restrictie Schaal (GARS): een handleiding [Measurement of disability in activities of daily living and instrumental activities of daily living with the Groning Activity Restriction Scale (GARS): a guideline]. Groningen: Rijksuniversiteit Groningen, Noordelijk Centrum voor Gezondheidsvraagstukken, 1993.

31. Hayes V, Morris J, Wolfe C, Morgan M. The SF-36 health survey questionnaire: is it suitable for use with older adults? Age Ageing 1995;24:120-125.

32. Kempen GIJM, Miedema I, Ormel J, Molenaar W. The assessment of disability with the Groningen Activity Restriction Scale. Conceptual framework and psychometric properties. Soc Sci Med 1996;33:1601-1610.

33. Lawton MP. Lawton's PGC Morale Scale 2003 [cited 2009 November 10]. Available from: http://www.abramsoncenter.org/pri/documents/PGC_morale_scale.pdf.

34. Lawton MP, Brody EM. Assessment of older people: self-maintaining and instrumental activities of daily living. Gerontologist 1969;9:179-186.

35. Van Breukelen GJP, Candel MJJM, Berger MPF. Relative efficiency of unequal versus equal cluster sizes in cluster randomized and multicentre trials. Stat Med 2007;26:2589-2603.

36. Rosnow RL, Rosenthal R. Statistical procedures and the justification of knowledge in psychological science. Am Psychol 1989;44:1276-1284.

37. Metzelthin SF, Van Rossum E, De Witte LP, Ambergen AW, Hobma SO, Siper W, et al. Effectiveness of interdisciplinary primary care approach to reduce disability in community dwelling frail older people: cluster randomised controlled trial. Br Med J 2013;347:f5264.

38. Blom JW, Den Elzen WPJ, Van Houwelingen AH, Heijmans M, Stijnen T, Van den Hout WB, et al. Effectiveness and cost-effectiveness of a proactive, goal-oriented, integrated care model in general practice for older people. Integrated Systematic Care for older People-the ISCOPE study. Eur Geriatr Med 2013;4:S168-S169.

39. Melis RJF, Van Eijken MIJ, Teerenstra S, Van Achterberg T, Parker SG, Borm GF, et al. A randomized study of a multidisciplinary program to intervene on geriatric syndromes in vulnerable older people who live at home (Dutch EASYcare study). J Gerontol 2008;63A:283-290. 
40. de Stampa M, Vedel I, Buyck J-F, Lapointe L, Bergman H, Beland F, et al. Impact on hospital admissions of an integrated primary care model for very frail elderly patients. Arch Gerontol Geriatr 2014;58:350355.

41. Ploeg J, Brazil K, Hutchison B, Kaczorowski J, Dalby DM, Goldsmith $\mathrm{CH}$, et al. Effect of preventive primary care outreach on health related quality of life among older adults at risk of functional decline: randomised controlled trial. Br Med J 2010;340:1480.

42. Nolte E, Knai C, Hofmarcher M, Conklin A, Erler A, Elissen A, et al. Overcoming fragmentation in health care: chronic care in Austria, Germany and the Netherlands. Health Economics, Policy and Law 2012;7:125-146.

43. Stijnen MMN, Jansen MWJ, Duimel-Peeters IGP, Vrijhoef HJM. Nurse-led home visitation programme to improve health-related quality of life and reduce disability among potentially frail communitydwelling older people in general practice: a theory-based process evaluation. BMC Fam Pract, 2014;15:173.

44. Stijnen MMN, Vrijhoef HJM, Duimel-Peeters IGP, Jansen MWJ. Formulation of and adherence to a care plan for potentially frail community-dwelling older people by practice nurses in the Netherlands. J Nurs Educ Pract 2014;4:34-43.

45. Hasson $\mathrm{H}$, Blomberg $\mathrm{S}$, Dunér A. Fidelity and moderating factors in complex interventions: a case study of a continuum of care program for frail elderly people in health and social care. Implement Sci 2012;7:23.

46. Rose G. Sick individuals and sick populations. Int J Epidemiol 1985;14:32-38.

47. Stijnen MMN, Van Hoof MS, Wijnands-Hoekstra IYM, Guldemond-Hecker Y, Duimel-Peeters IGP, Vrijhoef HJM, et al. Detected health and well-being problems following comprehensive geriatric assessment during a home visit among community-dwelling older people: who benefits most? Fam Pract 2014;31:333-340.

48. Fletcher AE, Price GM, Ng ESW, Stirling SL, Bulpitt CJ, Breeze E, et al. Population-based multidimensional assessment of older people in UK general practice: a cluster-randomised factorial trial. Lancet 2004;364:1667-1677.

49. Büla CJ, Bérod AC, Stuck AE, Alessi CA, Aronow HU, Santos-Eggimann B, et al. Effectiveness of preventive in-home geriatric assessment in well functioning, community-dwelling older people: secondary analysis of a randomized trial. J Am Geriatr Soc 1999;47:389-395.

50. Stuck AE, Kane RL. Whom do preventive home visits help? J Am Geriatr Soc 2008;56:561-563.

51. Sarkisian CA, Hays RD, Mangione CM. Do older adults expect to age successfully? The association between expectations regarding aging and beliefs regarding healthcare seeking among older adults. J Am Geriatr Soc 2002;50:1837-1843.

52. Evans D. Hierarchy of evidence: a framework for ranking evidence evaluating healthcare interventions. J Clin Nurs 2002;12:77-84.

53. Clark J. Preventive home visits to elderly people. Their effectiveness cannot be judged by randomised controlled trials. Br Med J 2001;323:708.

54. Van Heuvelen MJG, Hochstenbach JBM, Brouwer WH, De Greef MHG, Zijlstra GAR, Jaarsveld E, et al. Differences between participants and non-participants in an RCT on physical activity and psychological interventions for older persons. Aging Clin Exp Res 2005;17:236-245. 


\section{Detected health and well-being problems following comprehensive geriatric assessment during a home visit among community-dwelling older people: who benefits most?}

Published as:

Stijnen MMN, Van Hoof MS, Wijnands-Hoekstra IYM, Guldemond-Hecker Y, DuimelPeeters IGP, Vrijhoef HJM, Jansen MWJ. Detected health and well-being problems following comprehensive geriatric assessment during a home visit among communitydwelling older people: who benefits most? Fam Pract, 2014;13:333-340. 


\section{ABSTRACT}

\section{Background}

Preventive home visits including comprehensive geriatric assessment for potentially frail older people are increasingly implemented in general practice. It remains unclear how to select older people who benefit most from it.

\section{Objectives}

To determine which community-dwelling older people benefit most from a comprehensive geriatric assessment by a practice nurse during a home visit in terms of detected problems.

\section{Methods}

A cross-sectional study in 45 general practices in the Netherlands. Practice nurses visited 562 randomly selected older people (aged $\geq 75$ years) and 1,180 purposefully selected based on the following criteria: last visit to general practice > 6 months ago; partner or child(ren) deceased within past 12 months; cognitive or psychosocial functioning unknown to general practitioner (GP); $\geq 2$ chronic conditions; uses $\geq 5$ medications; and/or living alone.

\section{Results}

Mean age of older people was 82.50 years, $65.50 \%$ was female. More problems were detected among women, higher age groups, those living alone, and the less educated (all $\mathrm{P}<0.001$ ). Overall, more problems were detected in purposefully selected older people than in randomly selected older people $(P<0.001)$. Selection of older people with $\geq 2$ chronic conditions and those using $\geq 5$ medications resulted in more detected problems in general (both $\mathrm{P}<0.05$ ).

\section{Conclusion}

Although the findings are in favour of purposeful selection, observed differences in detected problems between the two selection procedures are relatively small. GPs should at least target older people with $\geq 2$ chronic conditions, using $\geq 5$ medications, being female, of an older age, living alone, and the less educated. 


\section{INTRODUCTION}

With the global rise in the number of older people, government policies need to deliver appropriate health systems that support the ability of older people to remain healthy and independent. ${ }^{1}$ In line with this, many preventive interventions have been developed with the ultimate goal to maintain independent living and to prevent or delay disability among community-dwelling older people. ${ }^{2,3}$ Especially preventive home visitation programmes for potentially frail community-dwelling older people have received considerable attention in the last decades.

An element of preventive home visitation programmes that has been defined as important in eliciting beneficial effects on patient outcomes is the comprehensive geriatric assessment. ${ }^{4}$ Elsawy and Higgins ${ }^{5}$ define a comprehensive geriatric assessment as "... a multidimensional, multidisciplinary assessment designed to evaluate an older person's functional ability, physical health, cognition and mental health, and socio-environmental circumstances." As such, the assessment aids in the organisation and coordination of individually appropriate care and well-being services, stimulation of self-management, as well as in monitoring and evaluation during follow-up. Preventive home visits including comprehensive geriatric assessment are increasingly implemented in primary care and community settings. The importance of addressing older people's needs is acknowledged and this should be accomplished by self-management support and individualised care, with the general practitioner (GP) as the central care provider responsible for early detection of health and well-being issues. ${ }^{6-8}$

An often-asked question is which older people tend to benefit most from a comprehensive geriatric assessment in terms of detected problems. Illife and Orrell ${ }^{9}$ suggest to identify common unmet needs by means of a focused, brief assessment or a two-staged process instead of a comprehensive but time-consuming multidimensional assessment among all older people. Targeting specific groups of older people is also topic of discussion in studies investigating the effects of home visits in general. For example, beneficial effects of home visits were only found among older people at low risk of functional decline ${ }^{10}$ or the effects were more pronounced among the youngerold ${ }^{4}$, while others contradict these findings and state that home visits are equally beneficial for the general population of older people. ${ }^{11}$ Further research is needed to assist healthcare professionals working in community and/or primary care in deciding whether and how to select specific groups of older people that benefit most from a comprehensive geriatric assessment.

The present study aims to determine which community-dwelling older people (aged $\geq 75$ years) benefit most from a comprehensive geriatric assessment by a primary care professional during a home visit in terms of detected problems. Data are used from the implementation of the [G]OLD home visitation programme ('Getting OLD the 
healthy way') in 45 general practices in the south of the Netherlands. Due to various ways in which general practices selected older people for the home visits, the influence of different selection criteria on the yield of the comprehensive geriatric assessment in terms of detected problems is examined.

\section{METHODS}

\section{Study design and setting}

A cross-sectional design was applied. Data were derived from 45 general practices located in two regions in the south of the Netherlands: 23 general practices in 'Maastricht-Heuvelland' and 22 general practices in 'Parkstad'.

\section{General practices}

Between January 2010 and April 2012, 45 out of 143 general practices in the two regions (31.47\%) implemented the [G]OLD home visitation programme. A prerequisite for participation was the availability of a practice nurse (PN) with sufficient time for care for older people. In the Netherlands, PNs are employed by the GP and they were introduced to relieve the GP's tasks by offering screening, treatment, care and education to chronically ill patients. Increasingly, care for older people is delegated to the $\mathrm{PN}$, due to their familiarity with a patient-centred, holistic approach in providing care. All 47 PNs from participating general practices were trained in performing the comprehensive geriatric assessment during a home visit and organising follow-up care.

\section{Selection of older people}

Community-dwelling older people aged 75 years and older were eligible to receive a home visit by the PN. Those not living independently, on a waiting list for admission to a nursing home or home for older people, under close medical supervision (chemotherapy, chronic haemodialysis or other therapies posing a high burden on the person), and the terminally ill were excluded. In addition, general practices applied one of the two following approaches in selecting older people for a home visit: (1) random selection or (2) purposeful selection. The random selection procedure was applied by thirteen general practices ( 7 from 'Parkstad'; 6 from 'Maastricht-Heuvelland') who agreed to participate in the intervention group of a quasi-experimental trial. ${ }^{12}$ After taking into account the amount of time PNs could dedicate to care for older people and the eligibility criteria, 1,817 older people $(47.95 \%)$ were randomly excluded. The remaining 1,972 older people were approached by means of an information letter and 
consent form and 721 older people (36.56\%) consented to participate. In total, 131 older people were not visited at home for a comprehensive geriatric assessment by a PN due to time constraints of the PN ( $n=42)$, they had declined participation $(n=29)$, deceased $(n=14)$, illness $(n=14)$, moved or switched general practice $(n=13)$, admission to nursing home $(n=7)$, admission to home for older people $(n=2)$, or unknown reason $(n=10)$. Of the remaining 590 older people, data are included in this study from 562 randomly selected older people for whom complete data were available in the electronic patient record. The other 32 general practices not involved in the quasiexperimental trial (17 from 'Maastricht-Heuvelland'; 15 from 'Parkstad') applied the purposeful selection procedure. At the start of the [G]OLD-project, an expert panel consisting of among others GPs, decided on the following criteria for the purposeful selection of older people, based on their personal experiences in daily practice and available scientific evidence ${ }^{13}$ : last visit to general practice $>6$ months ago; partner or child(ren) deceased within past 12 months; cognitive or psychosocial functioning unknown to GP; has $\geq 2$ chronic conditions; uses $\geq 5$ medications (polypharmacy); and living alone. The panel reached consensus that these criteria are expected to be indicative of older people in whom more problems will be detected. Based on the GP's knowledge of the older patients and a review of the GP Information System by the PN, each general practice created a list of older people who fulfilled one or more of the selection criteria. These older people were approached by the PN by phone or regular mail to invite them for a comprehensive geriatric assessment during a home visit. Once PNs finished the list, they could use the same procedure to create a new list of older people to visit. The PNs conducted 1,180 home visits between January 2010 and April 2012. The exact response rate of older people cannot be determined, as there are no records of the total number of people selected and approached by PNs. However, our earlier work in a similar context showed that $88.24 \%$ of those purposefully invited by the PN for a home visit agreed to participate. ${ }^{14}$ Complete data were available in the electronic patient record from all 1,180 purposefully selected older people.

\section{Data collection}

During the home visit, PNs administered the [G]OLD-instrument: a comprehensive geriatric assessment comprising evidence-based and practice-based tests and questions covering 30 topics related to the older person's physical, psychological, cognitive, and social functioning, as well as lifestyle and medication use (for a detailed overview, see Table 8.1). It assists the PN in uncovering early signs of decline or in identifying existing health and/or well-being problems that may threaten the ability of older people to grow old at home. Both GPs and PNs judged the [G]OLD-instrument to be useful for application during the home visit. ${ }^{14}$ 
Table 8.1 Content of the [G]OLD-instrument

\begin{tabular}{|c|c|c|}
\hline Topic $^{a}$ & Operationalisation & Cut-off point \\
\hline $\begin{array}{l}\text { Decline in general health } \\
\text { status }^{\text {b }}\end{array}$ & $\begin{array}{l}\text { Health in general }(1 \text { item SF- } 20)^{15} \text {; health status } \\
\text { now compared to one year ago }(1 \text { item SF-36) }\end{array}$ & $\begin{array}{l}\mathrm{N} / \mathrm{A} \text {; health status somewhat } \\
\text { worse or much worse than one } \\
\text { year age }\end{array}$ \\
\hline Complaints $^{b}$ & $\begin{array}{l}\text { Whether person experienced any complaints in } \\
\text { the past } 1 \text { or } 2 \text { months; three most important } \\
\text { complaints }\end{array}$ & N/A \\
\hline Polypharmacy & Number of prescribed drugs & Using $\geq 5$ medications \\
\hline $\begin{array}{l}\text { Discrepancy medication } \\
\text { use }\end{array}$ & $\begin{array}{l}\text { Comparison registered medication (prescription) } \\
\text { with actual medication use }\end{array}$ & $\begin{array}{l}\text { Discrepancy between registered } \\
\text { and actual medication use }\end{array}$ \\
\hline Dependency in (I)ADL & $\begin{array}{l}\text { GARS }^{17}: 18 \text { items on the ability to perform } \\
\text { activities of daily living (ADL) or instrumental } \\
\text { activities of daily living (IADL) }\end{array}$ & $\begin{array}{l}\text { Scored 'only with assistance' or } \\
\text { 'independent, but with great } \\
\text { effort' on at least one of the } \\
18 \text { items }\end{array}$ \\
\hline Urine incontinence & Undesired loss of urine in past 3 months & Yes, undesired loss of urine \\
\hline Faecal incontinence & Undesired loss of faeces in past 3 months & Yes, undesired loss of faeces \\
\hline Impaired sight & $\begin{array}{l}\text { Currently experiencing problems in daily living } \\
\text { because of impaired sight; decrease in eyesight } \\
\text { in the past } 6 \text { months } \\
\text { Difficulty to read newspaper; difficulty to } \\
\text { recognise face at } 4 \text { meters distance }{ }^{18}\end{array}$ & $\begin{array}{l}\text { Scored 'yes' on at least one of } \\
\text { the } 4 \text { items }\end{array}$ \\
\hline Impaired hearing & $\begin{array}{l}\text { Currently experiencing problems in daily living } \\
\text { because of impaired hearing; decreased hearing } \\
\text { in the past } 6 \text { months; environment indicates } \\
\text { impaired hearing }\end{array}$ & $\begin{array}{l}\text { Scored 'yes' on at least one of } \\
\text { the } 3 \text { items }\end{array}$ \\
\hline Overweight & Body Mass Index (BMI) - overweight & $\mathrm{BMI}>27$ \\
\hline Underweight & Body Mass Index (BMI) - underweight & $\mathrm{BMI}<20$ \\
\hline Undesired weight loss & $\begin{array}{l}\text { Whether person experienced undesired weight } \\
\text { loss in past 3-6 months }\end{array}$ & Yes, undesired weight loss \\
\hline Decreased appetite & $\begin{array}{l}\text { Whether person experienced a loss of appetite } \\
\text { in the past month }\end{array}$ & Yes, loss of appetite \\
\hline Systolic BP > 140 mm Hg & $\begin{array}{l}\text { Measurement of systolic blood pressure in } \\
\mathrm{mmHg}\end{array}$ & $\mathrm{BP}_{\text {syst }}>140 \mathrm{mmHg}$ \\
\hline Diastolic BP > 90 mm Hg & $\begin{array}{l}\text { Measurement of diastolic blood pressure in } \\
\mathrm{mmHg}\end{array}$ & $\mathrm{BP}_{\text {diast }}>90 \mathrm{mmHg}$ \\
\hline $\begin{array}{l}\text { Insufficient physical } \\
\text { activity }\end{array}$ & $\begin{array}{l}\text { Whether person is physically active on average } \\
30 \text { minutes per day, at least } 5 \text { days per week }\end{array}$ & $\begin{array}{l}\text { No, physically active below the } \\
\text { norm }\end{array}$ \\
\hline Smoking & Whether person smokes & Yes, smokes \\
\hline Excessive alcohol use & $\begin{array}{l}\text { Number of glasses of alcohol person drinks per } \\
\text { occasion (including two additional items to } \\
\text { obtain details on drinking behaviour) }\end{array}$ & $\begin{array}{l}\text { Drinks } 3 \text { or more glasses of } \\
\text { alcohol per occasion }\end{array}$ \\
\hline
\end{tabular}




\begin{tabular}{|c|c|c|}
\hline Topic $^{\text {a }}$ & Operationalisation & Cut-off point \\
\hline Memory problems & $\begin{array}{l}\text { Mini-cog test - mental status assessment of older } \\
\text { adults }^{19} \text { : clock-drawing test (scoring options: } \\
0 \text { (poor clock) to } 5 \text { (perfect clock)) and repeating } \\
\text { three words }\end{array}$ & $\begin{array}{l}\text { Incorrectly repeating all } 3 \text { words } \\
\text { (irrespective of score on clock- } \\
\text { drawing test) OR } 1 \text { or } 2 \text { words } \\
\text { incorrect, but score on clock- } \\
\text { drawing test of } 4 \text { or less }\end{array}$ \\
\hline Anxiety & $\begin{array}{l}\text { Part A of Hospital Anxiety and Depression Scale } \\
(\text { HADS })^{20}-7 \text { items }\end{array}$ & Score $\geq 8$ (range: $0-21$ ) \\
\hline Depressive complaints & $\begin{array}{l}5 \text { items Geriatric Depression Scale (GDS) }{ }^{21} \text { : } \\
\text { dissatisfaction; boredom; feelings of } \\
\text { helplessness; prefers to stay at home; feelings of } \\
\text { worthlessness }\end{array}$ & Score $\geq 2$ (range: $0-5$ ) \\
\hline Personality disorder & $\begin{array}{l}3 \text { items from Gerontologic Personality Disorders } \\
\text { Scale }(G P S)^{22} \text { : intention to end life; suffering } \\
\text { from nervousness, tension; used sleeping pills } \\
\text { in the past }\end{array}$ & Score $\geq 2$ (range: $0-3$ ) \\
\hline $\begin{array}{l}\text { Fall incidents in past } 6 \\
\text { months }\end{array}$ & Number of falls past 6 months & $\geq 1$ falls past 6 months \\
\hline Concerned about falling & Whether person is ever concerned about falling & $\begin{array}{l}\text { Often or very often concerned } \\
\text { to fall }\end{array}$ \\
\hline $\begin{array}{l}\text { Avoidance of activities } \\
\text { due to fear or falling }\end{array}$ & $\begin{array}{l}\text { Whether person avoids certain activities due to } \\
\text { fear of falling }\end{array}$ & $\begin{array}{l}\text { Often or very often avoidance of } \\
\text { activities }\end{array}$ \\
\hline Decreased mobility & Get-up and go test ${ }^{23}$ & Score $\geq 3$ (range: $0-5$ ) \\
\hline $\begin{array}{l}\text { Unsatisfied with daily } \\
\text { routines }\end{array}$ & $\begin{array}{l}\text { Whether person is satisfied with daily activities } \\
\text { or routines }\end{array}$ & $\begin{array}{l}\text { Mostly not or totally not } \\
\text { satisfied with daily activities or } \\
\text { routines }\end{array}$ \\
\hline Loneliness & 6 -item loneliness scale ${ }^{24}$ & Score $\geq 3$ (range: $0-6$ ) \\
\hline $\begin{array}{l}\text { Burdened by informal } \\
\text { care giving }\end{array}$ & $\begin{array}{l}\text { Whether person experiences informal care } \\
\text { giving as a burden }\end{array}$ & $\begin{array}{l}\text { Sometimes, often or always } \\
\text { burdened by informal care } \\
\text { giving }\end{array}$ \\
\hline $\begin{array}{l}\text { Concerned about } \\
\text { informal caregiver }\end{array}$ & $\begin{array}{l}\text { Whether person is concerned about the informal } \\
\text { caregiver }\end{array}$ & $\begin{array}{l}\text { Yes, concerned about informal } \\
\text { caregiver }\end{array}$ \\
\hline $\begin{array}{l}\text { Absence of social } \\
\text { network }\end{array}$ & Whether a social network is present & No, social network is absent \\
\hline Financial problems & $\begin{array}{l}\text { Whether person had financial problems in the } \\
\text { past } 12 \text { months }\end{array}$ & Yes, financial problems \\
\hline Quality of life ${ }^{b}$ & Self-reported quality of life (grade 1-10) & Grade of 5 or less \\
\hline
\end{tabular}

N/A means not applicable.

${ }^{a}$ In the same order as they appear in the [G]OLD-instrument.

${ }^{\mathrm{b}}$ Not included in prevalence rates or sum scores in the current study, since these topics do not represent a distinctive problem and may be related to other topics in the [G]OLD-instrument. 
PNs used a paper format during the home visit and afterwards, the results of the assessment were registered by the PN or the Primary Healthcare Organisation in the electronic patient record used for chronic disease management. Relevant data on behalf of the current study were extracted anonymously from the electronic patient record by the responsible Primary Healthcare Organisation of each region.

\section{Data analysis}

The prevalence of individual problems detected through the comprehensive geriatric assessment was determined using the pre-specified cut-off points of the [G]OLDinstrument. Chi-square tests were performed to compare differences in prevalence rates between randomly and purposefully selected older people. The total number of detected problems was calculated and used as an outcome measure to test for differences between randomly selected and purposefully selected older people, as well as to examine differences in socio-demographic characteristics (gender, age, living status, and level of education). Because the data did not meet the assumption of normality (based on the Kolmogorov-Smirnov and Shapiro-Wilk statistics) and relevant transformations did not improve the distributions, non-parametric tests were used (i.e., Mann-Whitney U-test and Kruskall-Wallis H-test). In addition, for the purposefully selected older people, Mann-Whitney U-tests were conducted to examine per selection criterion to what extent the number of detected problems differed between older people selected based on that criterion versus those selected based on the other criteria. In all Mann-Whitney U-tests, the distributions compared had homogeneous variances, but different shapes. Therefore, means ranks and corresponding interquartile ranges are reported for non-normally distributed continuous variables. Percentages are reported for categorical variables. Data were analysed using the statistical package SPSS for windows, version 19 (SPSS, Chicago, IL). Differences were considered statistically significant at $\mathrm{P}<0.05$.

\section{RESULTS}

\section{Socio-demographic characteristics}

In total, 1,742 home visits were conducted by the PNs. The average number of home visits performed per general practice was 38.71 visits ( $S D=32.57$; range: $1-129)$. Mean age of the visited older people was 82.50 years (SD=4.52; range: $75.01-97.72$ ), $65.50 \%$ was female, $60.15 \%$ lived alone, $50.71 \%$ had completed secondary education or lower to middle professional education, and $56.13 \%$ was widowed (Table 8.2). Compared to 
the randomly selected older people, purposefully selected older people were significantly older $(+1.72 \mathrm{yr})$, were more often female $(+12.11 \%)$, more often lived alone $(+11.19 \%)$, and more often had no education or only primary education $(+7.57 \%)$. No significant regional differences were found with respect to these socio-demographic variables and, therefore, the two regions were combined in subsequent analyses. The proportion of randomly selected to purposefully selected older people was approximately $1: 2$ in both regions.

Table 8.2 Socio-demographic characteristics of older people enrolled according to selection procedure and region

\begin{tabular}{|c|c|c|c|c|c|c|c|}
\hline & Overall & $\begin{array}{l}\text { Randomly } \\
\text { selected }\end{array}$ & $\begin{array}{l}\text { Purposefully } \\
\text { selected }\end{array}$ & $\begin{array}{c}\mathrm{P} \text { - } \\
\text { value }\end{array}$ & $\begin{array}{l}\text { Maastricht- } \\
\text { Heuvelland }\end{array}$ & Parkstad & $\begin{array}{c}\text { P- } \\
\text { value }\end{array}$ \\
\hline Number of subjects (n) & 1,742 & $562(32.26)$ & $1,180(67.74)$ & - & $703(40.36)$ & $1,039(59.64)$ & - \\
\hline Age (years) & & & & $<0.001$ & & & 0.676 \\
\hline Mean (SD) & $82.50(4.52)$ & $81.33(4.26)$ & $83.05(4.54)$ & & $82.44(4.59)$ & $82.54(4.48)$ & \\
\hline Range & $75.01-97.72$ & $75.02-95.52$ & $75.01-97.72$ & & $75.02-97.72$ & $75.01-96.66$ & \\
\hline Gender, n (\%) & & & & $<0.001$ & & & 0.641 \\
\hline Male & $601(34.50)$ & $240(42.70)$ & 361 (30.59) & & $238(33.85)$ & $363(34.94)$ & \\
\hline Female & $1,141(65.50)$ & $322(57.30)$ & $819(69.41)$ & & $465(66.15)$ & $676(65.06)$ & \\
\hline Living status, $\mathbf{n}(\%)$ & & & & $<0.001$ & & & 0.183 \\
\hline Living alone & $1,028(60.15)$ & $288(52.55)$ & $740(63.74)$ & & $406(58.25)$ & $622(61.46)$ & \\
\hline Living with partner or others & $681(39.85)$ & $260(47.46)$ & $421(36.26)$ & & $291(41.75)$ & $390(38.54)$ & \\
\hline Level of education, $n$ (\%) & & & & 0.011 & & & 0.214 \\
\hline $\begin{array}{l}\text { No education or primary } \\
\text { education }\end{array}$ & $674(39.60)$ & $190(34.48)$ & $484(42.05)$ & & $258(37.61)$ & $416(40.94)$ & \\
\hline $\begin{array}{l}\text { Secondary education or lower } \\
\text { to middle professional } \\
\text { education }\end{array}$ & $863(50.71)$ & $302(54.81)$ & $561(48.74)$ & & $353(51.46)$ & $510(50.20)$ & \\
\hline Higher professional education & $165(9.69)$ & $59(10.71)$ & $106(9.21)$ & & 75 (10.93) & $90(8.86)$ & \\
\hline Marital status, n (\%) & & & & NA & & & NA \\
\hline Married & $584(33.62)$ & $242(43.29)$ & $342(29.03)$ & & $248(35.28)$ & $336(32.49)$ & \\
\hline Living together, unmarried & $31(1.78)$ & $10(1.79)$ & $21(1.78)$ & & $15(2.13)$ & $16(1.55)$ & \\
\hline Single, widowed & $975(56.13)$ & $270(48.30)$ & $705(59.85)$ & & $373(53.06)$ & $602(58.22)$ & \\
\hline Single, never married & $147(8.46)$ & $37(6.62)$ & $110(9.34)$ & & $67(9.53)$ & $80(7.74)$ & \\
\hline
\end{tabular}

NA, not applicable 


\section{Prevalence of individual problems detected through comprehensive geriatric assessment}

The prevalence of individual problems detected through comprehensive geriatric assessment is presented in Table 8.3. Overall, the most frequently detected problems among older people were discrepancy between registered and actual medication use (86.29\%), dependency in (instrumental) activities of daily living ((I)ADL) (81.92\%), polypharmacy (48.95\%), high systolic blood pressure $(47.46 \%)$, and decreased mobility (42.05\%). The least common problems were financial problems $(2.68 \%)$, unsatisfied with daily routines $(4.12 \%)$, underweight $(4.68 \%)$, faecal incontinence $(5.45 \%)$, and absence of a social network (8.17\%). For nearly all individual problems, the prevalence rates were higher among purposefully selected older people compared to the randomly selected older people, and for 16 out of 30 problems the difference was statistically significant $(\mathrm{P}<0.05)$. In particular, all five psychological/mental problems and more than half of the physical problems were significantly more prevalent among the purposefully selected older people. Only overweight $\left(\mathrm{BMI}>27 \mathrm{~kg} / \mathrm{m}^{2}\right.$ ) showed a significant result in the opposite direction.

\section{Median number of detected problems by socio-demographic characteristics}

Table 8.4 illustrates that more problems were detected in purposefully selected older people than in randomly selected older people (mean rank 932.91 vs. 738.97, $\mathrm{P}<0.001)$. Overall, significantly more problems were detected among women than men (mean rank 923.08 vs. $770.85, \mathrm{P}<0.001$ ). The number of detected problems was also significantly affected by age $(P<0.001)$. Jonckheere-Terpstra's test revealed a significant trend, meaning that the number of detected problems increased with age $(P<0.001)$. Further, significantly more problems were detected among older people who live alone compared to those who live with a partner or others $(P<0.001)$. Finally, the number of detected problems was significantly affected by level of education $(P<0.001)$. With increasing level of education, the number of detected problems decreased $(P<0.001)$. The same pattern of findings is found among both randomly selected and purposefully selected older people, indicating that the findings were not influenced by any significant differences in socio-demographic characteristics between the two groups. 


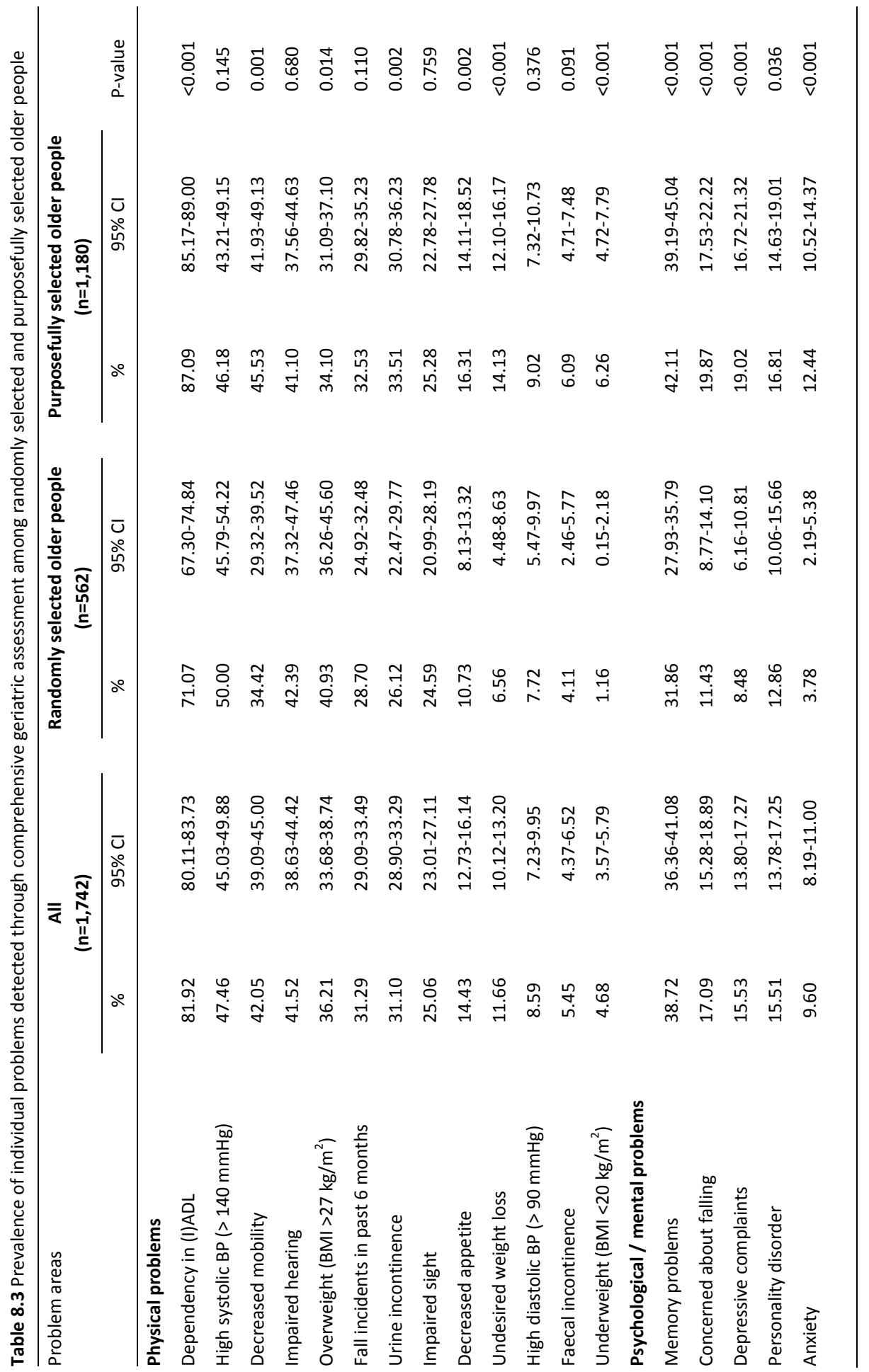




\section{CHAPTER 8}

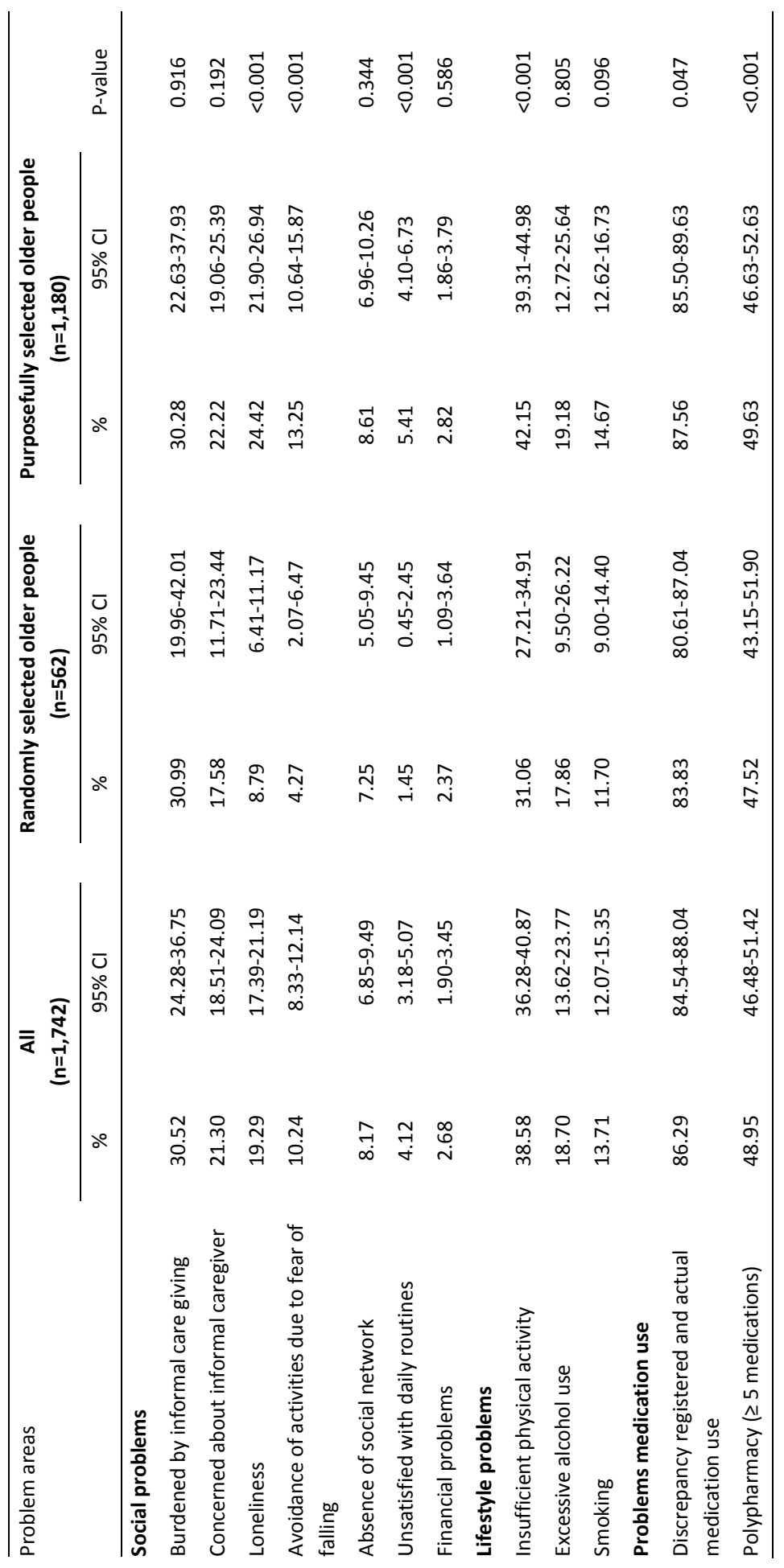




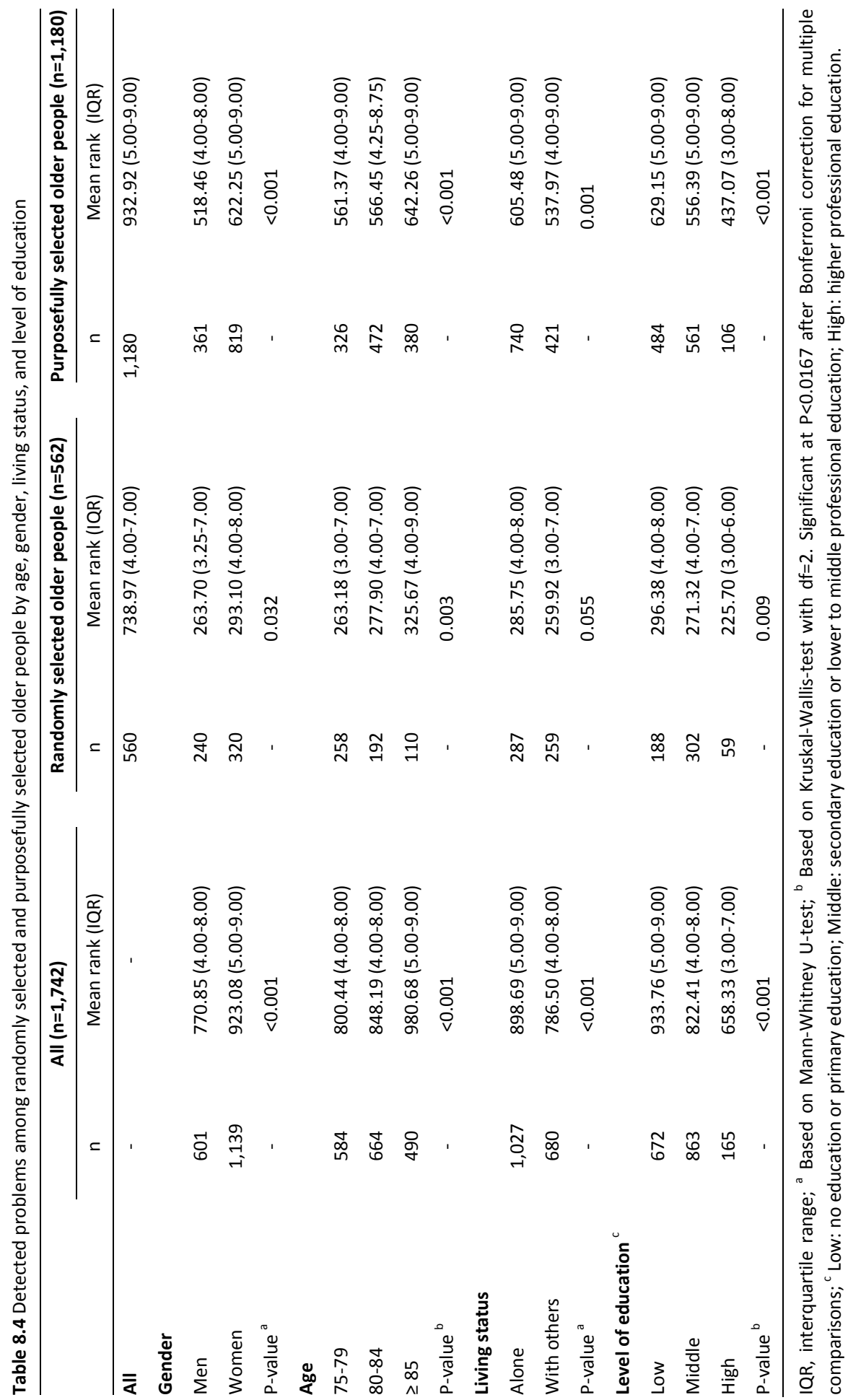




\section{Purposefully selected older people}

Per selection criterion, Table 8.5 compares the number of detected problems among older people selected based on that criterion to older people selected based on one of the other criteria. The number of detected problems was significantly lower among older people whose last contact with the general practice was > 6 months ago compared to those selected based on one of the other criteria (mean rank 293.77 vs. $379.25, \mathrm{P}=0.001$ ). Older people with $\geq 2$ chronic conditions experienced significantly more problems compared to older people selected based on one of the other criteria (mean rank 454.68 vs. $365.92, \mathrm{P}<0.05$ ). Finally, the number of detected problems was significantly higher among older people who used $\geq 5$ medications than among those selected based on one of the other criteria (mean rank 446.40 vs. $366.09, \mathrm{P}<0.05$ ).

Table 8.5 Detected problems per individual selection criterion applied by GPs among purposefully selected older people $(n=739)$

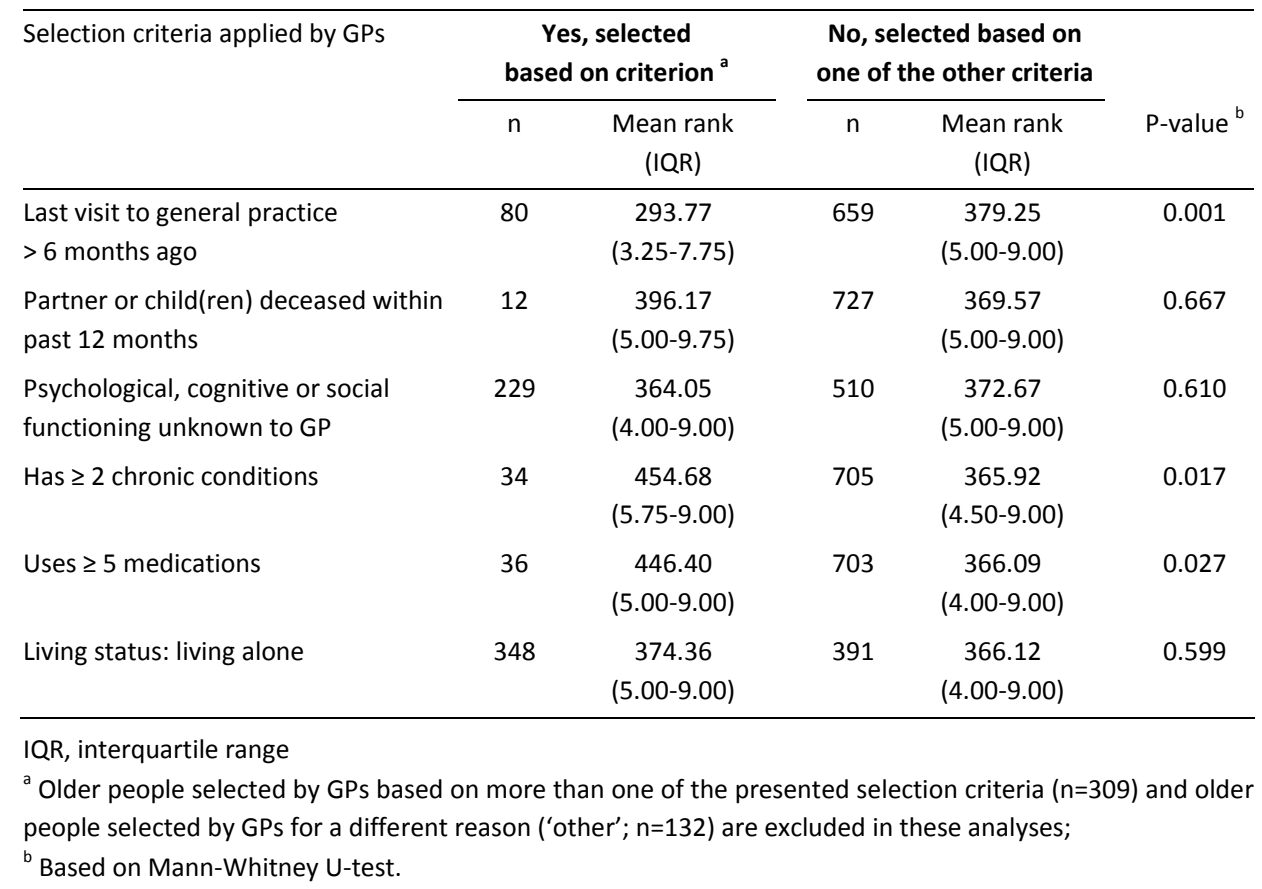




\section{DISCUSSION}

This study reports on a large sample of 1,742 community-dwelling older people in the south of the Netherlands who were selected randomly versus purposefully for a comprehensive geriatric assessment by a PN during a home visit. Overall, the findings imply that purposefully selecting older people for a home visit results in more detected problems compared to randomly selecting older people.

This finding supports those who advocate the need to target preventive home visits to appropriate subpopulations that are most likely to benefit from it. ${ }^{25}$ From a cost-benefit perspective, targeting individuals at high-risk is most appealing to general practices in order to make effective use of limited resources (i.e., time of the PN). Furthermore, it is in line with the GPs' usual approach to care delivery, as GPs may not be motivated to intervene in situations without a specific request for help. On the other hand, any problems disclosed among randomly selected older people may deserve the GP's attention depending on the older person's needs and therefore they are not negligible. The more population-based approach of randomly selecting older people may also ultimately result in benefits to the older population as a whole, which characterises a public health perspective to prevention among older people. ${ }^{26}$ Random selection of older people is applied in an ongoing quasi-experimental controlled trial on the effects of the [G]OLD home visitation programme on patient outcomes ${ }^{12}$ and results will become available in due course. In the large Medical Research Council (MRC) trial in UK general practices, targeted assessment had no additional benefit in terms of mortality, admission to hospital or institution, or quality of life compared to a broad, universal assessment among older people. ${ }^{27}$ Although we found statistically significant differences between randomly selected and purposefully selected older people in the prevalence of individual problems and total number of detected problems, the differences are relatively small. Thus, the question arises whether these differences are clinically relevant enough to opt for purposeful selection of older people in favour of random selection.

In selecting older people who benefit most from comprehensive geriatric assessment in terms of detected problems, besides socio-demographic characteristics (being female, older age, living alone, and a lower educational level), two selection criteria applied by GPs proved useful as well: having $\geq 2$ chronic conditions and using $\geq 5$ medications (polypharmacy). Interestingly, we found that significantly fewer problems were detected among older people who had not been in contact with the general practice for more than six months. This suggests that these are the more healthy older people without a need to consult their GP and that they do not represent a group of older people with multiple problems who refuse or avoid seeking care/help. Finally, although more problems were detected among older people living alone, when applied by GPs 
as a selection criterion it did not lead to significantly more problems being detected. Of all older people living alone, GPs selected only a subset in which they expected problems to be found. Moreover, it is unclear to what extent GPs can accurately estimate which older people are most likely to benefit from a comprehensive geriatric assessment.

Unfortunately, we were not able to determine the yield of the comprehensive geriatric assessment in terms of newly detected problems previously unknown to the GP or relevant problems that require new or ongoing care according to the GP and in agreement with the older person's needs and wishes. Nevertheless, previous studies $^{28,29}$ support the ability of the comprehensive geriatric assessment to disclose unknown problems in general practice among random samples of older people. Moreover, the randomly selected older people included in this study may not represent a truly random sample of all older people aged 75 years and older within general practices / the community. The recruitment procedure can have introduced volunteer bias, in that the healthier older people are more likely to participate in the trial. ${ }^{30}$ Consequently, the differences between randomly and purposefully selected older people may be smaller than observed in the present study.

\section{CONCLUSION}

Despite more problems being detected among purposefully selected older people, the small differences do not overtly support purposeful selection over random selection of older people for a comprehensive geriatric assessment. From a public health perspective, it seems equally important to approach randomly selected older people. Recent studies demonstrated that preventive home visits including a comprehensive geriatric assessment are feasible in general practice and acceptable to older patients. ${ }^{14,28}$ Yet, some GPs may prefer a less time-consuming way of obtaining a complete overview of older people's health and well-being. In that case, the present study suggests that GPs should at least prioritise older people based on whether they have $\geq 2$ chronic conditions, use $\geq 5$ medications, are female, are of an older age group, live alone, and have a lower educational level. 


\section{REFERENCES}

1. Rechel B, Doyle $\mathrm{Y}$, Grundy E, McKee M. How can health systems respond to population ageing? Copenhagen: World Health Organization;2009.

2. Beswick AD, Rees K, Dieppe P, Ayis S, Gooberman-Hill R, Horwood J, et al. Complex interventions to improve physical function and maintain independent living in elderly people: a systematic review and meta-analysis. Lancet 2008;371:725-735.

3. Daniels R, Van Rossum E, De Witte LP, Kempen GIJM, Van den Heuvel W. Interventions to prevent disability in frail community-dwelling elderly: a systematic review. BMC Health Serv Res 2008;8:278.

4. Huss A, Stuck AE, Rubenstein LZ, Egger M, Clough-Gorr KM. Multidimensional preventive home visit programs for community-dwelling older adults: a systematic review and meta-analysis of randomized controlled trials. J Gerontol A Biol Sci Med Sci 2008;63A:298-307.

5. Elsawy B, Higgins KE. The geriatric assessment. Am Fam Physician 2011;83:48-56.

6. Boeckxstaens P, De Graaf P. Primary care and care for older persons: position paper of the European Forum for Primary Care. Qual Prim Care 2011;19:369-389.

7. Dutch College of General Practitioners. NHG-Standpunt Toekomstvisie Huisartsenzorg. Huisartsgeneeskunde voor ouderen [Statement of the NHG, Future of General Practitioner Care. General practice medicine for older people]. Utrecht: NHG;2007.

8. Vass M, Avlund K, Hendriksen C, Philipson L, Riis P. Preventive home visits to older people in Denmark. Why, how, by whom, and when? Z Gerontol Geriatr 2007;40:209-216.

9. Iliffe $S$, Orrell M. Identifying unmet health needs in older people: comprehensive screening is not the answer. Br J Gen Pract 2006;56:404-406.

10. Stuck AE, Minder CE, Peter-Wüest I, Gillmann G, Egli C, Kesselring A, et al. A randomized trial of inhome visits for disability prevention in community-dwelling older people at low and high risk for nursing home admission. Arch Intern Med 2000;160:977-986.

11. Elkan R, Kendrick D, Dewey M, Hewitt M, Robinson J, Blair M, et al. Effectiveness of home based support for older people: systematic review and meta-analysis. Br Med J 2001;323:719-725.

12. Stijnen MMN, Duimel-Peeters IGP, Jansen MWJ, Vrijhoef HJM. Early detection of health problems in potentially frail community-dwelling older people by general practices - project [G]OLD: design of a longitudinal, quasi-experimental study. BMC Geriatr 2013;13:7.

13. Puts MT, Lips $P$, Deeg DJ. Static and dynamic measures of frailty predicted decline in performancebased and self-reported physical functioning. J Clin Epidemiol 2005;58:1188-1198.

14. Stijnen MMN, Jansen MWJ, Vrijhoef HJM, Duimel-Peeters IGP. Development of a home visitation programme for the early detection of health problems in potentially frail community-dwelling older people by general practices. Eur J Ageing 2013;10:49-60.

15. Kempen GIJM, Brilman El, Heyink JW, Ormel J. Het meten van de algemene gezondheidstoestand met de MOS Short-Form General Health Survey (SF-20): een handleiding [Measurement of general health status with the MOS Short-Form General Health Survey (SF-20): a guideline]. Groningen: Rijksuniversiteit Groningen, Noordelijk Centrum voor Gezondheidsvraagstukken, 1995.

16. Van der Zee KI, Sanderman R. Het meten van de algemene gezondheidstoestand met de RAND-36: een handleiding [Measurement of general health status with the RAND-36: a guideline]. Groningen: Rijksuniversiteit Groningen, Noordelijk Centrum voor Gezondheidsvraagstukken, 1993.

17. Kempen GIJM, Doeglas DM, Suurmeijer TPBM. Het meten van problemen met zelfredzaamheid op verzorgend en huishoudelijk gebied met de Groningen Activiteiten Restrictie Schaal (GARS): een handleiding [Measurement of disability in activities of daily living and instrumental activities of daily living with the Groning Activity Restriction Scale (GARS): a guideline]. Groningen: Rijksuniversiteit Groningen, Noordelijk Centrum voor Gezondheidsvraagstukken, 1993. 
18. Van Sonsbeek JLA. Methodological and substantial aspects of the OECD indicator of functional limitations. Maandbericht Gezondheid (CBS) 1988;88:4-17.

19. Borson S, Scanlan J, Brush M, Vitaliano P, Dokmak A. The Mini-Cog: A cognitive 'vital signs' measure for dementia screening in multi-lingual elderly. Int J Geriatr Psychiatry 2000;15:1021-1027.

20. Zigmond AS, Snaith RP. The hospital anxiety and depression scale. Acta Psychiatr Scand 1983;67:361370.

21. Hoyl MT, Alessi CA, Harker JO, Josephson KR, Pietruszka FM, Koelfgen M, et al. Development and testing of a five-item version of the Geriatric Depression Scale. J Am Geriatr Soc 1999;47:873-878.

22. Van Alphen SP, Engelen GJ, Kuin Y, Hoijtink H, Derksen JJ. Constructie van een schaal voor de signalering van persoonlijkheidsstoornissen bij ouderen [Construction of a scale to detect personality disorders in older people]. Tijdschr Gerontol Geriatr 2004;35:186-195.

23. Mathias S, Navak US, Isaacs B. Balance in elderly patients: the "get-up and go" test. Arch Phys Med Rehabil 1986;67:387-389.

24. De Jong Gierveld J, Van Tilburg T. A 6-Item scale for overall, emotional, and social loneliness. Confirmatory tests on survey data. Res Aging 2006;28:582-598.

25. Rubenstein LZ, Stuck AE. Preventive home visits for older people: defining criteria for success. Age Ageing 2001;30:107-109.

26. Rose G. Sick individuals and sick populations. Int J Epidemiol 1985;14:32-38.

27. Fletcher AE, Price GM, Ng ESW, Stirling SL, Bulpitt CJ, Breeze E, et al. Population-based multidimensional assessment of older people in UK general practice: a cluster-randomised factorial trial. Lancet 2004;364:1667-1677.

28. Lucchetti G, Granero AL. Use of comprehensive geriatric assessment in general practice: results from the 'Senta Pua' project in Brazil. Eur J Gen Pract 2011;17:20-27.

29. Piccoliori G, Gerolimon E, Abholz H. Geriatric assessment in general practice using a screening instrument: is it worth the effort? Results of a South Tyrol Study. Age Ageing 2008;37:647-652.

30. Van Heuvelen MJG, Hochstenbach JBM, Brouwer WH, De Greef MHG, Zijlstra GAR, Jaarsveld E, et al. Differences between participants and non-participants in an RCT on physical activity and psychological interventions for older persons. Aging Clin Exp Res 2005;17:236-245. 
General discussion 



\section{INTRODUCTION}

The [G]OLD home visitation programme was developed to offer general practices an instrument for the early detection of health problems in potentially frail older people embedded within a protocol for organising proactive care for older people that would both be feasible and useful for application in daily practice. Care for older people within general practices was redesigned by applying components of the Chronic Care Model $(\mathrm{CCM})^{1}$ and the Guided Care Model, $^{2}$ resulting in a comprehensive protocol that guided general practices in planning and executing geriatric care. The main elements of the [G]OLD-protocol were a comprehensive geriatric assessment by the practice nurse (PN) during a home visit, post-discussion with the general practitioner (GP) to discuss the findings, the formulation of a care and treatment plan (if needed) in line with the older person's needs and wishes, and multidisciplinary care management and followup over time according to the goals as formulated in the care and treatment plan. The [G]OLD home visitation programme was implemented in general practices in the regions 'Maastricht-Heuvelland' and 'Parkstad' in the south of the Netherlands.

The overall aim of the work presented in this dissertation was to assess the feasibility and effectiveness of the [G]OLD home visitation programme for potentially frail community-dwelling older people (aged $\geq 75$ years) in general practice. As a result, the individual studies in this dissertation described in consecutive steps the process of the development of the [G]OLD home visitation programme, followed by investigating whether the [G]OLD home visitation programme was implemented as planned, and effective in terms of an improvement in health-related quality of life and a reduction in disability among community-dwelling older people. Furthermore, it was examined which community-dwelling older people benefit most from the [G]OLD comprehensive geriatric assessment in terms of detected health and/or well-being problems. The specific findings along with the strengths and limitations of each study have been addressed in the chapters of this dissertation. This general discussion summarises the main findings from these studies and discusses the overall theoretical and methodological considerations, as well as the implications for further research and for practice. At the end of the chapter, an overall conclusion is provided.

\section{MAIN FINDINGS}

\section{Preliminary feasibility}

Twenty-one general practices participated in a pilot study (Chapter 2) and 240 community-dwelling older people (aged $\geq 75$ years) were visited at home by the PN. A mixed-model design was applied to evaluate the initial feasibility of the home visits, 
practical usefulness of the [G]OLD comprehensive geriatric assessment instrument, and to what extent the different steps of the [G]OLD home visitation programme were performed according to the protocol. Both GPs and PNs judged the [G]OLD home visitation programme to be feasible in daily practice, though there were concerns about the time investment being disproportionate compared to the costs reimbursed by health insurers. Nevertheless, they believed it improved their quality of care. Older people were satisfied about the duration of the home visit as well as the questions asked as part of the [G]OLD comprehensive geriatric assessment. The [G]OLD comprehensive geriatric assessment instrument was considered useful by both GPs and PNs, mostly because it allowed for obtaining a complete overview of an older person's health and well-being status. Yet improvements were needed, such as clearer cut-off points for individual tests in the assessment. Furthermore, the [G]OLD-instrument was able to identify meaningful health or well-being problems for which follow-up actions were needed according to the formulated care and treatment plan. Finally, protocol deviations (e.g., skipping items of the [G]OLD-instrument, inaccurate or inadequate reporting of the care and treatment plan by the $\mathrm{PN}$ ) offered insight into the necessary improvements for optimal performance of the [G]OLD home visitation programme beyond the pilot phase. By testing the intervention in a pilot setting, we obtained advance indications of threats to adherence to the intervention protocol and whether instruments as part of the intervention were appropriate, which are among the advantages of conducting a pilot study. ${ }^{3}$

\section{Implementation in general practice}

A comprehensive process evaluation plan (Chapter 3) was developed to examine whether the [G]OLD home visitation programme was implemented as planned and the extent to which general practices redesigned their care delivery from reactive, diseaseoriented care towards proactive, patient-oriented care. The process evaluation plan was designed according to the seven key elements of process evaluation as proposed by Saunders and colleagues: ${ }^{4}$ fidelity (quality of implementation), dose delivered (completeness), dose received (exposure), dose received (satisfaction), reach (participation rate), recruitment, and context. Furthermore, the element 'context' was explored into more detail using the Normalisation Process Model, $^{5}$ which provides a conceptual framework for understanding the factors that affect the success or failure of a complex intervention in primary care in relation to the workability and integration of the intervention components. A prospectively developed theory-based process evaluation plan is relatively uncommon and therefore, we presented several arguments for conducting a process evaluation alongside a clinical trial, as well as arguments for developing a detailed process evaluation plan. 
A prospective process evaluation plan, underpinned by a theoretical framework, and using a mixed-method approach to data collection allowed for a more thoroughly conducted process evaluation (Chapter 4 ). The majority of process evaluation data were collected from GPs, PNs, and older people from the 13 general practices (out of 24) who implemented the [G]OLD home visitation programme (intervention practices). Both GPs and PNs evaluated the [G]OLD home visitation programme positively, because it resulted in more attention for older people within their general practice, closer collaboration between the GP and PN, and a comprehensive picture of older people's functioning and social network. The older people themselves were satisfied with the home visit, as it offered them the ability to express their daily concerns, such as impairments associated with ageing. Overall, the [G]OLD home visitation programme did not have major shortcomings in itself, but the delivery offered room for improvement. The [G]OLD home visitation programme was delivered completely according to protocol to only $38.0 \%$ of the 721 elderly participants. Several threats to complete delivery of the intervention were identified. First, few new health and/or well-being problems were detected among older people, which caused certain elements of the intervention protocol to be non-applicable. According to GPs and PNs, this could be attributed to the relatively healthy older people who consented to participate in research and were thus visited, suggesting inadequate reach. Second, perceived lack of time was a prominent issue raised that could explain why various elements of the [G]OLD home visitation programme were not delivered as planned. The time investment and corresponding available financial reimbursement by health insurers were considered disproportionate compared to the benefits in terms of detected problems. A solution proposed by GPs and PNs to reduce the time investment of the [G]OLD home visitation programme was to target the intervention to those older people who are defined by further research as most likely to benefit from it. Overall, general practices successfully redesigned their care delivery from reactive towards proactive care, yet the threats to complete delivery of the intervention as mentioned before may cause them to relapse into their old way of providing reactive care to older people.

Both the findings of the pilot study as well as the process evaluation showed that PNs often did not formulate care and treatment plans or they formulated them inappropriately (e.g., incomplete, no concrete goals). Therefore, an additional crosssectional, mixed-model study was set up (Chapter 5) to investigate PNs' actions and views regarding (1) the formulation of a care and treatment plan for older people and (2) the adherence to the care and treatment plan and monitoring during follow-up of older people in whom health and/or well-being problems had been detected. The findings showed that only $27.8 \%$ of the problems identified through the [G]OLD comprehensive geriatric assessment were included in a care and treatment plan by the PN. According to the PN, the main reason for not including an identified problem in the 
plan was because it was an existing problem and/or action had already been undertaken. Both patient-related factors (e.g., the older person's wishes) and problem-specific factors (e.g., perceived seriousness of the problem) influenced the formulation of care plans. If problems were registered in the care and treatment plan, PNs mostly adhered to it as shown by the finding that $62.9 \%$ of the registered problems were dealt with according to PNs.

\section{Effectiveness}

We planned a longitudinal study (Chapter 6 and Chapter 7) to investigate the effectiveness of the [G]OLD home visitation programme. A quasi-experimental design was applied which refrained from creating artificial situations that might hinder the translation of research findings into practice ${ }^{6-8}$ and allowed for routinisation of working methods in daily practice to ensure sustainability of the [G]OLD intervention protocol over time. ${ }^{9}$ Twenty-four general practices participated of which 13 general practices redesigned their care delivery to older people and implemented the [G]OLD home visitation programme (intervention practices), whereas 11 general practices provided usual care to older people (control practices). Community-dwelling older people aged $\geq 75$ years were eligible to participate. In total, 4,588 eligible older people were approached for participation and 1,737 older people (37.9\%) consented to participate. Primary outcome measures were health-related quality of life and disability, measured by means of a self-reported questionnaire at baseline and after 6,12 , and 18-months follow-up. Data of 586 older people in the intervention group and 941 older people in the control group were available for analysis. Mixed regression models only showed a marginally significant effect of the [G]OLD home visitation programme on role limitations due to physical problems at 6 months and 18 months follow-up compared to the control group. No statistically significant effects in favour of the intervention group were found on all other outcome measures and time points.

\section{Selection of older people for comprehensive geriatric assessment}

A cross-sectional study (Chapter 8 ) was conducted in 45 general practices in the south of the Netherlands who implemented the [G]OLD home visitation programme. The aim was to determine which community-dwelling older people (aged $\geq 75$ years) benefit most from the [G]OLD comprehensive geriatric assessment during a home visit in terms of detected health and/or well-being problems. Thirteen general practices who participated in the quasi-experimental trial randomly selected community-dwelling older people, which resulted in the inclusion of 562 older people in this study. The remaining 32 general practices chose a different selection procedure by purposefully selecting older people who fulfilled one of the following criteria according to the GP: last visit to general practice $>6$ months ago; partner or child(ren) deceased within past 
12 months; cognitive or psychosocial functioning unknown to GP; $\geq 2$ chronic conditions; uses $\geq 5$ medications and/or living alone. The findings revealed that even though observed differences in detected problems between the two selection procedures were relatively small, more problems were detected in purposefully selected older people than in randomly selected older people. Among the purposeful selection criteria, most problems were detected in older people with $\geq 2$ chronic conditions and those using $\geq 5$ medications. Furthermore, more problems were detected among women, higher age groups, those living alone and those less educated.

\section{THEORETICAL CONSIDERATIONS}

Along with the development of various preventive home visitation programmes over the last decades, there has been an ongoing discussion about their effectiveness in older people. ${ }^{10-12}$ The present work showed that the [G]OLD preventive home visitation programme did not result in convincing significant effects on the primary outcomes health-related quality of life and disability and the secondary outcome attitude towards ageing in older people within general practice (Chapter 7). A recent systematic review by Mayo-Wilson and colleagues ${ }^{13}$ stated that preventive home visits are not consistently associated with effects on mortality or independent living, although some individual programmes in specific settings may produce beneficial effects. Before discussing the methodological concerns associated with investigating the effects of preventive home visits, the theoretical assumptions underlying the [G]OLD home visitation programme deserve some further consideration. They have been introduced in Chapter 1, namely the theoretical framework underlying the [G]OLD home visitation programme, content of the [G]OLD home visitation programme, the screening approach, and implementation in general practice.

\section{Theoretical framework}

A strength of the [G]OLD home visitation programme is that a theoretic framework guided intervention design. As described in Chapter 3 and Chapter 6, the CCM and the Guided Care model were used for this purpose. Although originally developed to improve the management of existing chronic illnesses in primary care, ${ }^{1}$ the CCM has been expanded to make it applicable to preventive care as well. ${ }^{14,15}$ As an example, the CCM has been successfully applied in addressing health risk behaviours, such as smoking, excessive alcohol use, unhealthy dietary patterns, and physical inactivity. ${ }^{16}$ However, the merit of applying the CCM to organise proactive care to community-dwelling frail older persons in the primary care setting remains to be investigated. ${ }^{17}$ So far, Guided Care seems to be the only evidenced-based model that translated components of the 
CCM in an effort to transform care for vulnerable older people with multiple chronic conditions and complex care needs. ${ }^{18,19}$ Furthermore, whereas the CCM only encompasses general ideas for change and is not a directly replicable intervention, Guided Care offers a phased approach how to build them into an intervention protocol. Therefore, the phases as proposed by the Guided Care model were adapted to the Dutch general practice setting. Given the lack of evidence regarding the usefulness of CCM to change care delivery for older people in Dutch primary care, the appropriateness of the theoretical framework underlying the [G]OLD home visitation programme for advancing preventive geriatric care is open for discussion. Nonetheless, our findings showed that the [G]OLD home visitation programme was feasible in general practice (Chapter 2 and Chapter 4), but failed to result in convincing significant effects at the patient level (Chapter 7). This implies that applying CCM components by means of the phases of the Guided Care model is a promising approach for general practices, however the effects on health outcomes in older people remain to be established.

\section{Content of the [G]OLD home visitation programme}

Besides the usefulness of the theoretical framework in general, another issue relates to whether intervention content was strong enough to achieve sustainable change in care delivery to older people by general practices. The CCM was not adopted as a whole, since the focus was only on four out of six components: delivery system design, decision support, clinical information systems, and community resources (Chapter 3 and Chapter 6), and not on healthcare organisation and self-management support. Coleman and colleagues ${ }^{20}$ found that the best results are obtained when practices make changes across multiple components of the CCM. On the other hand, adoption of all CCM components poses difficulties in term of increased complexity, while single components of the CCM can be implemented rather easily in primary care. ${ }^{21}$ There is no consensus to date on a minimally required set of CCM components to be included. ${ }^{20}$ Therefore, we cannot claim that adoption of the CCM as a whole would have increased the likelihood of obtaining favourable outcomes in older people.

For the [G]OLD home visitation programme, delivery system design was the most important CCM component, as this entailed redesigning care delivery for older people within general practice from reactive to proactive care. However, some general practices were prone to relapse into reactive care delivery to older people after the intervention period (Chapter 4). This may have been stimulated by the predominantly reactive approach to care delivery in Dutch primary care. On the other hand, as suggested by research into quality improvement in primary care, general practices should be regarded as complex adaptive systems ${ }^{22}$ in which change is the result of a complex interplay between factors inside (e.g., motivation of GPs) and outside the general practice (e.g., opportunity for delivering proactive care to older people as facilitated by 
health insurers). ${ }^{23}$ This would necessitate more attention to the process of redesigning care within general practices to sustain proactive care delivery to older people. Merely implementing a proactive intervention such as the [G]OLD home visitation programme may have been overly simplistic to reach this aim. More attention should have been paid to offering GPs and PNs the necessary knowledge and skills for causing a shift in thinking and working, from reactively addressing single problems to proactively addressing multiple problems detected among older people.

Apart from the theoretical framework used, intervention content can also be judged in the light of current knowledge in the field of preventive home visitation programmes. Various single elements were included in the intervention protocol of the [G]OLD home visitation programme that have been postulated as promising elements of preventive home visitation programmes or interventions targeting disability prevention in frail older people. ${ }^{11,24,25}$ Due to the complex nature of home visitation programmes and concomitant heterogeneity in reported studies, disentangling the independent effects of intervention elements on patient outcomes is an ongoing challenge. ${ }^{10,13}$ Moreover, to our knowledge no attention has been paid to reinforcing and/or counterproductive interactions between intervention elements, restricting the advancement of knowledge regarding the optimal combination of intervention components. Nevertheless, there is convincing evidence that multicomponent interventions rather than single-component interventions are most successful in the preservation of physical functioning and maintaining independent living in community-dwelling older people. ${ }^{26,27}$ As a result, some uncertainty remains with respect to whether the content of the [G]OLD home visitation programme contained the right mixture of effective ingredients that are minimally required to achieve an improvement in healthrelated quality of life and a reduction in disability.

Finally, there have been inconsistencies in previous work with respect to whether older people in poor health or those in better health benefit most from home visitation programmes. ${ }^{10,28}$ It has been argued that multidimensional preventive interventions targeting multiple and potentially co-existing problems and risks should be offered to older people when they are in the early and thus reversible stages of the disability process, whereas the more disabled and frail older people benefit from intensive multidimensional interventions targeting specific problems. ${ }^{29-31}$ This suggests that follow-up actions should be adjusted to the specific disability stage in which older people reside. The [G]OLD home visitation programme allowed PNs to tailor appropriate follow-up actions to the specific needs as identified by means of the [G]OLD comprehensive geriatric assessment. However, this process may have been disturbed by other factors, such as the unwillingness of older people to undertake follow-up actions for detected problems (Chapter 5), inadequate skills of the PN to adequately apply the [G]OLD comprehensive geriatric assessment for the detection of problems and imple- 
ment follow-up actions to address these problems, ${ }^{30}$ or a lack of (effective) services available to refer older people to who present themselves with certain problems. As such, the lack of effects may not only be attributed to the content of the [G]OLD home visitation programme, but also to these additional factors that are essential for the adequate performance of the [G]OLD home visitation programme and its ability to reach effects on health-related outcomes in older people.

\section{Screening approach}

In the [G]OLD home visitation programme, potentially frail community-dwelling older people aged 75 years and older were considered eligible to receive a comprehensive geriatric assessment. This approach to screening most closely resembles populationbased screening. The case for routine assessment of those aged 75 years and over in general practice has been subject of discussion for many years. One of the largest trials in the field demonstrated no additional benefit of population-based screening over target assessment of older people in general practice. ${ }^{32}$ As a result, comprehensive geriatric assessment in the general population of older people is being discouraged. ${ }^{33 \text {, }}$ ${ }^{34}$ However, studies have revealed that a comprehensive geriatric assessment is able to disclose unmet needs among older people in general practice. ${ }^{35-37}$ In addition, GPs participating in our pilot study indicated that they are confronted with a heterogeneous group of older people in daily practice, which requires a structured, multidimensional comprehensive geriatric assessment (Chapter 2 ). Though this partly justifies the chosen screening approach, we were not able to tackle the administrative burden and considerable time investment associated with the performance of a comprehensive geriatric assessment (Chapter 2 and Chapter 4). Furthermore, a disadvantage of the chosen approach is that older people have been included who possibly resided in various stages of the frailty continuum or suffered from different degrees of disability, thereby diluting any preventive effects of the home visitation programme on healthrelated quality of life and disability.

Another option proposed in the literature is a two-staged approach in which a short screening instrument is used to detect frailty in older people (first stage) and those considered frail are then submitted to a more elaborate comprehensive geriatric assessment (second stage), followed by individually appropriate care and/or well-being interventions. $^{33,38}$ To date, no census exists regarding the optimal tool for the detection of frailty in older people in general practice. ${ }^{38,39}$ Moreover, the implementation of this two-staged approach in general practice poses additional challenges related to for example determining the appropriate cut-off point for continuation to the second stage. $^{40}$

We therefore examined a third option, namely targeting the comprehensive geriatric assessment to older people most likely to benefit from it in terms of the number 
of detected problems. Purposeful selection of older people (those who fulfill one of the following criteria: suffering from $\geq 2$ chronic conditions, using $\geq 5$ medications, female, the oldest-olds, living along, and the less educated) slightly favoured randomly visiting all over-75-year-olds (Chapter 8). However, more work is needed on this topic, for example to identify the combined effect of selection criteria on the number of detected problems, the relative weight of each criterion, and other criteria that GPs can easily determine based on their GP Information System.

Finally, the [G]OLD comprehensive geriatric assessment instrument itself deserves some attention. Instead of solely focusing on the physical aspects of frailty as common under the frailty phenotype, ${ }^{41}$ in this work we adopted a multidimensional perspective in which physical, mental, psychological, and social functioning was covered by the [G]OLD-instrument. This also allowed GPs and PNs to obtain a complete overview of older people's functioning with respect to the various domains. The [G]OLDinstrument was based on the existing TRAZAG instrument ${ }^{42,43}$ (TRAZAG is the Dutch abbreviation of 'Transmuraal Zorg Assessment Geriatrie') and was further adapted to make it applicable to general practices (Chapter 2). Given the complexity of examining the psychometric properties of such a multidimensional instrument composed of various individual tests and questions, we opted for including as much as possible existing evidence-based tests. Yet, most of these tests were not developed for individual diagnosis, making their usefulness for this purpose questionable. However, we noticed that GPs and PNs used the [G]OLD-instrument as just one of the sources of information for formulating a care and treatment plan besides patient-related factors (e.g., the older person's wishes) and problem-specific factors (e.g., perceived seriousness of the problem) (Chapter 5).

\section{Implementation in general practice}

Although general practices have been considered the ideal setting for organising preventive care for older people, ${ }^{44,45}$ few studies examined the effectiveness of preventive home visitation programmes in general practice. In advancing knowledge in this area of research, the present work investigated both the effectiveness as well as the feasibility of the [G]OLD home visitation programme in the general practice setting. The equal emphasis on a theory-based process evaluation besides the effect evaluation resulted from the notion that complex healthcare quality improvement interventions can only be properly understood if the evaluation focusses on the interconnections between content, application, context, and outcomes, as proposed by Walshe. ${ }^{46}$ In explaining the absence of significant effects of the [G]OLD home visitation programme, we can conclude from our process evaluation that implementation failure was probably more likely than intervention failure (Chapter 7). It is known that preventive interventions diffuse relatively slowly, caused by the increased time span for ob- 
taining results and associated delay in rewards from adopting a preventive intervention. ${ }^{47}$ It becomes even more challenging when considering the implementation of complex interventions that go beyond mere adoption and diffusion, because of the necessity to embed them in settings that are dynamic and complex in themselves. ${ }^{48,49}$ This is the case for the implementation of the [G]OLD home visitation programme in general practices. Thus, GPs and PNs might have needed more time for properly experimenting with a complex intervention such as the [G]OLD home visitation programme before it could be performed in its most optimal form. Furthermore, due to the introduction of a chronic care management programme regarding vascular risk management in general practices at the time of the implementation of the [G]OLD home visitation programme, GPs and PNs experienced time restrictions (Chapter 7). As a result, the learning process may have been disturbed by rapid adoption, ${ }^{50}$ resulting in suboptimal performance of certain elements of the [G]OLD home visitation programme protocol. Some PNs may have rushed through the performance of the home visits, thereby paying insufficient attention to the individual elements of the [G]OLD home visitation programme, such as the formulation of a care and treatment plan with clear directions for follow-up. In sum, this suggests that the process evaluation provided valuable information regarding the early phases of embedding the [G]OLD home visitation programme in general practice and practice redesign, yet the effect evaluation might have been performed too early.

In interpreting the findings, it should be kept in mind that the Netherlands is classified as having one of the most well-organised primary healthcare systems of European countries. ${ }^{44,51}$ Furthermore, primary care in the south of the Netherlands ('ZuidLimburg') is characterised by a high level of multidisciplinary cooperation compared to other Dutch regions, which facilitates coordination and the provision of coherent care. ${ }^{52}$ Consequently, it is reasonable to assume that citizens living in the south of Limburg already receive relatively high-quality care from their general practice. The room for improvement in patient outcomes might have been too limited due to possibly high baseline values, which may explain the lack of significant effects of the [G]OLD home visitation programme.

\section{METHODOLOGICAL CONSIDERATIONS}

The results of the different studies presented in this dissertation should also be interpreted in the light of several methodological considerations. Since specific methodological strengths and shortcomings of the individual studies have already been presented in the previous chapters, this section will address general methodological is- 
sues. These relate to the study design, participants, and outcome measures, which are discussed below.

\section{Study design}

The most appropriate study design for investigating the effectiveness of quality improvement interventions in healthcare, such as preventive home visitation programmes, has been subject of discussion. ${ }^{46,53}$ Most researchers rely on randomised controlled trials for studying effectiveness of interventions. We opted for a quasiexperimental design in which clusters (i.e., general practices) were allocated to either the intervention group or the control group. In choosing an appropriate study design, we strived for obtaining a balance between optimal causal inference and refraining from creating artificial situations that may hinder care delivery in general practice (Chapter 3). The latter evolved out of the unwillingness of GPs to participate in a randomised design, because they did not want to withhold older people from receiving possibly good care. On the one hand, it might be argued that practice-based research is hindered by these practical obstacles. On the other hand, it underlines that caregivers strive to deliver the best possible care, which is the ultimate goal of quality improvement in healthcare.

The cluster quasi-experimental design also has some drawbacks. First, allocating of clusters (i.e., general practices) to either the intervention or control group minimised the risk of contamination at the cost of an increase in the required sample size and total number of general practices needed, thereby adding to the logistical complexity of the trial. ${ }^{54}$ Second, due to the absence of randomisation, internal validity is threatened. ${ }^{55}$ Systematic differences between the study groups may exist and as a result differences in the outcome may be attributed to other known and unknown prognostic factors besides the effect of the intervention. ${ }^{56}$ Furthermore, significant imbalances between the study groups in baseline variables were found. Although these were accounted for in the mixed regression models used for analysis (Chapter 7), these and other factors (e.g., related to regional differences between the intervention and control group) may still have a negative influence on the causal inferences that can be drawn from the results.

For the process evaluation a mixed-method design was used (Chapter 3 and Chapter 4), whereas the additional study to examine the formulation of and adherence to the care and treatment plan by practice nurses applied a mixed-model design (Chapter 5). Too often, the use of both qualitative and quantitative data is described erroneously under the general heading of mixed-methods. However, different approaches for combining qualitative and quantitative methods can be distinguished. ${ }^{57}$ In general, the term mixed-model studies refers to the mixing of both qualitative and quantitative research strands within or across the different stages of the research process (i.e., 
research objectives, data collection, data analysis or data interpretation). ${ }^{58}$ On the other hand, mixed-method studies involve the inclusion of a separate quantitative and qualitative phase in an overall research study. ${ }^{58}$ The work presented in this dissertation can therefore be described as one overall mixed-method study using a convergent parallel approach and, more specifically, a parallel databases design. ${ }^{57}$ Combining both qualitative and quantitative research allowed for obtaining a more detailed understanding of the subject under investigation, which justifies the time consuming nature of this type of designs. ${ }^{58}$ However, it becomes difficult when mixed method findings contradict one another, as was the case here. Yet, for a large part this can be attributed to the fact that the outcome measures of the effectiveness study are different from the outcomes measures of the process evaluation. Therefore, both data strands should be considered as fundamentally different research paradigms but complementary to one another and in that way crucial for more thoroughly investigating complex interventions. $^{59}$

\section{Participants}

One of the major strengths of our quasi-experimental controlled trial was a high number of included participants compared to other comparable studies in the field of preventive home visits. ${ }^{13}$ Although the cluster design required a higher sample size, we had sufficient power to detect differences in the primary outcome measure healthrelated quality of life. Nevertheless, participant recruitment suffered from a relatively high non-response, as only $37.9 \%$ of all 1,737 community-dwelling older people approached for participation consented to participate (Chapter 7). We initially expected a response rate of $50 \%$ (Chapter 6 ), which is realistic given existing evidence on the recruitment of community-dwelling older people for similar studies. ${ }^{60} \mathrm{~A}$ higher number of older people were approached for participation than originally planned, especially in the control group. The difficulty of recruiting older people for controlled trials has been recognised before. The only method we now used to improve the response rate, was offering non-responders additional verbal information about the study during a telephone call approximately one month after the information letter and informed consent had been sent. Other relevant methods that could have been included are involving family members in the consent process and providing additional information concerning the informed consent form, as older people may be afraid of the consequences of signing it. ${ }^{61}$

As a consequence of the low response rate, the representativeness of the study population is at stake. Adding to this, older people who agreed to participate were the young-old and they were less likely to be labelled as potentially frail by their GP (Chapter 7). Moreover, at all follow-up time points, retention was more likely among the young-old and those who reported better functioning in (instrumental) activities of 
daily living (Chapter 7). This implies that the study population consisted of the relatively healthy older people. As a result, we may conclude that [G]OLD home visitation programme does not work in the young-old with low levels of functional decline. Although it has been postulated earlier in this discussion that older people at low risk of functional decline benefit from multidimensional preventive interventions, this study population was probably too healthy, which influenced both the effect study (i.e., dilution of preventive effects), as well as the process evaluation (i.e., implementation failure due to low protocol adherence as certain elements of the [G]OLD home visitation programme were not applicable). It is generally recognised that selecting and retaining older people in research is one of the most important challenging aspects to consider while designing this type of studies. ${ }^{62}$

\section{Outcome measures}

For studying the effectiveness of the [G]OLD home visitation programme, we used validated questionnaires to assess the primary outcome measures health-related quality of life and disability at baseline, 6 months, 12 months, and 18 months follow-up (Chapter 6). Previous studies have examined the effectiveness of preventive home visits for older people on a range of different outcome measures. ${ }^{13}$ For the present work, generic outcome measures were chosen, while more individually relevant measures that allow for the evaluation of outcomes across different treatment goals ${ }^{63}$ could have been more sensitive for effect evaluation purposes. On the other hand, for informing public health policy it is more relevant to know the effects on outcomes measures such as health-related quality of life and disability. However, the follow-up period of 18 months may not have been long enough to establish effects on these distal outcome measures, though 18 months follow-up is a notable time period for reaching effects in the older population.

\section{IMPLICATIONS FOR RESEARCH}

Several overall implications for research of the work presented in this dissertation are worth mentioning here. In general, this dissertation adds to the available knowledge base that the effectiveness of preventive home visitation programmes for communitydwelling older people is at best uncertain. As advocated by Huss and colleagues, ${ }^{64}$ this type of intervention needs to be evaluated in the real clinical practice setting in which they are intended to function, in this case general practices. However, more research is needed regarding the effectiveness of home visitation programmes within general practices. Due to the complexity of this setting, many factors can influence the performance of home visitation programmes that are also inherently complex themselves. 
As recommended by the Medical Research Council (MRC) framework ${ }^{65}$ and concluded by the current work, future studies should use available evidence and appropriate theory for designing preventive home visitation programmes, pilot test them to reveal uncertainties in the design, followed by a comprehensive evaluation to assess their effectiveness, understand change processes, and determine cost-effectiveness. The latter was not done for the current home visitation programme, but undoubtedly deserves further attention as little is known regarding the effectiveness in relation to costs. ${ }^{25}$ However, it is not advised to design and evaluate another home visitation programme unless there is a clear justification for the likelihood of obtaining effects compared to the wealth of studies that have been conducted thus far.

With respect to the design of home visitation programmes, defining the most effective components of home visitation programmes as well as which older people benefit most from it, has been on the research agenda for over a decade. ${ }^{66}$ Although some progress has been made, many unclarities remain and this dissertation again emphasises that if future studies regarding home visitation programme are undertaken, these issues deserve special attention. This dissertation provided preliminary insight into which older people benefit most from the [G]OLD comprehensive geriatric assessment, yet more evidence is needed to appropriately advise healthcare professionals how best to select older people for a comprehensive geriatric assessment during a home visit to make effective use of the limited time of the PN.

With respect to the evaluation of home visitation programmes, the limitations of the current work regarding study design, selection of participants, assessment of outcome measures, as well as limitations defined in earlier studies in this field of research should be taken into consideration. More importantly, to advance evidence-based practice in public health, more practice-based evidence is required. ${ }^{8}$ The former viewpoint (i.e., evidence-based practice) is dominant among researchers who solely rely on randomised controlled trials for obtaining evidence regarding cause and effect. However, it may not be the most suitable design for examining the effectiveness of complex interventions that contain a complex mix of uncontrollable factors. ${ }^{53}$ Up to now, the majority of home visitation programmes did not incorporate a thoroughly conducted process evaluation to provide insight into the implementation and potential factors that may explain trial results beyond mere appreciation of the intervention by the target group and professionals involved. A shift in thinking is needed among purely quantitative researchers that a comprehensive process evaluation should always run parallel to the effectiveness study, as it provides equally important and even more practically relevant evidence for justifying the continuation of home visitation programmes in practice settings. 


\section{IMPLICATIONS FOR PRACTICE}

One of the most important practical implications of the work presented in this dissertation relates to current discussions regarding reforming long-term care financing in the Netherlands. Based on projections of future expenditure on long-term care, sustainable, efficient, and consumer-directed delivery of long-term care can only be guaranteed by reforming the current Dutch system of long-term care funding. ${ }^{67}$ Consequently, as of January 2015, most of the long-term care services currently covered by the Exceptional Medical Expenses Act (abbreviated as 'AWBZ') are transferred to the new national Health Insurance Act for curative health services (abbreviated as ' $Z V W$ ') and the remaining services (related to social support and participation) into the new Social Support Act (abbreviated as 'WMO'). ${ }^{67}$ Under the latter Act, responsibilities for assigning the remaining services are delegated to municipalities who strive to prevent long-term care and to facilitate independent living among older people. ${ }^{68}$ This type of reform also has consequences for primary care, as well-developed primary care systems can reduce the need for hospital and long-term care by early identification of health problems and needs. ${ }^{44}$ As a result of these developments, the need for general practices to implement effective care models to address the care needs of older people will continue to grow.

The [G]OLD home visitation programme offered general practices a stepwise approach for detecting and addressing health and/or well-being among potentially frail older people ( $\geq 75$ years) at an early stage. Although the [G]OLD home visitation programme was judged useful by general practices for delivering care to potentially frail older people, it did not have beneficial effects on older people's health-related quality of life and disability. Therefore, the dissemination and routine implementation of the [G]OLD home visitation programme in its current form is doubtful. More attention should be paid to improve the system of care surrounding an older person. This perspective is incorporated in recent discussion concerning whole person care provision that focusses on new models of person-centred, coordinated care provision for older people with complex needs to improve their health and well-being and maintain independent living in their own homes. Whole person care places the individual person and his carer at the central point of attention and supports independent living by, among others, technological innovations, shared decision-making, and a multidisciplinary team for collaborative care planning. ${ }^{69}$ For general practices this would mean that they need to extend their care services and re-align their work to act as one team together with local health services and social care services. 


\section{CONCLUSIONS}

The most important conclusions that can be drawn from the studies as presented in this dissertation, according to the objectives as specified in the general introduction (Chapter 1 ), are as follows:

1. The [G]OLD home visitation programme proved to be feasible in general practices;

2. The [G]OLD home visitation programme did not have any major shortcomings in itself, but suffered from implementation failure and the uncertain commitment of general practices towards sustained redesign in care delivery from reactive, disease-oriented care towards proactive, patient-oriented care;

3. The [G]OLD home visitation programme had no convincing significant effects on the primary outcomes health-related quality of life and disability, and the secondary outcome attitude towards ageing in community-dwelling older people;

4. The [G]OLD home visitation programme should preferably be targeted to purposefully selected older people, as most problems were detected by means of the [G]OLD comprehensive geriatric assessment in older people with $\geq 2$ chronic conditions, those using $\geq 5$ medications, women, higher age groups, those living alone, and the less educated.

In sum, based on available knowledge in the field of preventive home visits and the findings from our work, the routine dissemination and implementation of the [G]OLD home visitation programme in general practices in its current form is doubtful, as the effectiveness of home visitation programmes for community-dwelling older people is at best uncertain. However, considering the ageing of our population and concomitant healthcare reforms planned for the near future, the need for general practices to reorganise care for potentially frail older people from reactive to proactive care will persist. Therefore, the information obtained from the studies presented in this dissertation regarding the feasibility and effectiveness of the [G]OLD home visitation programme warrant further consideration before undertaking any comparable or adjusted home visitation programme for future community-dwelling older people ( $\geq 75$ years) within general practices or other multidisciplinary care service teams. 


\section{REFERENCES}

1. Bodenheimer T, Wagner EH, Grumbach K. Improving primary care for patients with chronic illness. J Am Med Assoc 2002;288:1775-1779.

2. Boult C, Karm L, Groves C. Improving chronic care: the "Guided Care" model. Perm J 2008;12:50-54.

3. Van Teijlingen ER, Hundley V. The importance of pilot studies. Nurs Stand 2002;16:33-36.

4. Saunders RP, Evans MH, Joshi P. Developing a process-evaluation plan for assessing health promotion program implementation: a how-to guide. Health Promot Pract 2005;6:134-147.

5. May CR. A rational model for assessing and evaluating complex interventions in health care. BMC Health Serv Res 2006;6:86.

6. Green LW, Glasgow RE. Evaluating the relevance, generalization, and applicability of research. Issues in external validation and translation methodology. Eval Health Prof 2006;29:126-153.

7. Glasgow RE, Green LW, Klesges LM, Fisher E, Goldstein MG, Hayman LL, et al. External validity: we need to do more. Ann Behav Med 2006;31:105-108.

8. Green LW. Making research relevant: if it is an evidence-based practice, where's the practice-based evidence? Fam Pract 2008;25:20-24.

9. Jansen M, Harting J, Ebben N, Kroon B, Stappers J, Van Engelshoven E, et al. The concept of sustainability and the use of outcome indicators. A case study to continue a successful health counselling intervention. Fam Pract 2008;25:32-37.

10. Elkan R, Kendrick D, Dewey M, Hewitt M, Robinson J, Blair M, et al. Effectiveness of home based support for older people: systematic review and meta-analysis. Br Med J 2001;323:719-725.

11. Stuck AE, Egger M, Hammer A, Minder CE, Beck JC. Home visits to prevent nursing home admission and functional decline in elderly people: systematic review and meta-regression analysis. J Am Med Assoc 2002;287:1022-1029.

12. Van Haastregt JCM, Diederiks JPM, Van Rossum E, De Witte LP, Crebolder HFJM. Effects of preventive home visits to elderly people living in the community: systematic review. Br Med J 2000;320:754-758.

13. Mayo-Wilson E, Grant S, Burton J, Parsons A, Underhill K, Montgomery P. Preventive home visits for mortality, morbidity, and institutionalization in older adults: a systematic review and meta-analysis. PLoS ONE 2014;9:e89257.

14. Barr VJ, Robinson S, Marin-Link B, Underhill L, Dotts A, Ravensdale D, et al. The expanded Chronic Care Model: an integration of concepts and strategies from population health promotion and the Chronic Care Model. Hosp Q 2003;7:73-82.

15. Glasgow RE, Orleans CT, Wagner EH, Curry SJ, Solberg LI. Does the Chronic Care Model serve also as a template for improving prevention? The Milbank Quarterly 2001;79:579-612.

16. Hung DY, Rundall TG, Tallia AF, Cohen DJ, Halpin HA, Crabtree BF. Rethinking prevention in primary care: applying the Chronic Care Model to address health risk behaviors. The Milbank Quarterly 2007;85:69-91.

17. Muntinga ME, Hoogendijk EO, Van Leeuwen KM, Van Hout HPJ, Twisk JWRVdH, H. E., Nijpels G, et al. Implementing the chronic care model for frail older adults in the Netherlands: study protocol of ACT (frail older adults: care in transition). BMC Geriatr 2012;12:19.

18. Boyd CM, Boult C, Shadmi E, Leff B, Brager R, Dunbar L, et al. Guided care for multimorbid older adults. Gerontologist 2007;47:697-704.

19. Boyd CM, Reider L, Frey K, Scharfstein D, Leff B, Wolff J, et al. The effects of Guided Care on the perceived quality of health care for multi-morbid older persons: 18-month outcomes from a clusterrandomized controlled trial. J Gen Intern Med 2010;25:235-242.

20. Coleman K, Austin BT, Brach C, Wagner EH. Evidence on the chronic care model in the new millennium. Health Aff (Millwood) 2009;28:75-85. 
21. Bodenheimer T, Wagner EH, Grumbach K. Improving primary care for patients with chronic illness. The Chronic Care Model, part 2. J Am Med Assoc 2002;288:1909-1914.

22. Stroebel CK, McDaniel RR, Crabtree BF, Miller WL, Nutting PA, Stange KC. How complexity science can inform a reflective process for improvement in primary care practices. Jt Comm J Qual Patient Saf 2005;31:438-446.

23. Cohen D, McDaniel RR, Crabtree BF, Ruhe MC, Weyer SM, Tallia AF, et al. A practice change model for quality improvement in primary care practice. J Healthc Manag 2004;49:155-168.

24. Daniels R, Metzelthin S, Van Rossum E, De Witte L, Van den Heuvel W. Interventions to prevent disability in frail community-dwelling older persons: an overview. Eur J Ageing 2010;7:37-55.

25. Markle-Reid M, Browne G, Weir R, Gafni A, Roberts J, Henderson SR. The effectiveness and efficiency of home-based nursing health promotion for older people: a review of the literature. Med Care Res Rev 2006;63:531-569.

26. Beswick AD, Rees K, Dieppe P, Ayis S, Gooberman-Hill R, Horwood J, et al. Complex interventions to improve physical function and maintain independent living in elderly people: a systematic review and meta-analysis. Lancet 2008;371:725-735.

27. Daniels R, Van Rossum E, De Witte LP, Kempen GIJM, Van den Heuvel W. Interventions to prevent disability in frail community-dwelling elderly: a systematic review. BMC Health Serv Res 2008;8:278.

28. Bouman A, Van Rossum E, Nelemans P, Kempen GIJM, Knipschild P. Effects of intensive home visiting programs for older people with poor health status: a systematic review. BMC Health Serv Res 2008;8:74.

29. Rubenstein LZ, Stuck AE. Preventive home visits for older people: defining criteria for success. Age Ageing 2001;30:107-109.

30. Stuck AE, Minder CE, Peter-Wüest I, Gillmann G, Egli C, Kesselring A, et al. A randomized trial of inhome visits for disability prevention in community-dwelling older people at low and high risk for nursing home admission. Arch Intern Med 2000;160:977-986.

31. Stuck AE, Kane RL. Whom do preventive home visits help? J Am Geriatr Soc 2008;56:561-563.

32. Fletcher AE, Price GM, Ng ESW, Stirling SL, Bulpitt CJ, Breeze E, et al. Population-based multidimensional assessment of older people in UK general practice: a cluster-randomised factorial trial. Lancet 2004;364:1667-1677.

33. De Lepeleire J, Iliffe S, Mann E, Degryse J. Frailty: An emerging concept for general practice. Br J Gen Pract 2009;59:e177-e182.

34. Iliffe $\mathrm{S}$, Orrell M. Identifying unmet health needs in older people: comprehensive screening is not the answer. Br J Gen Pract 2006;56:404-406.

35. Lucchetti G, Granero AL. Use of comprehensive geriatric assessment in general practice: results from the 'Senta Pua' project in Brazil. Eur J Gen Pract 2011;17:20-27.

36. Müller CA, Klaassen-Mielke R, Penner E, Junius-Walker U, Hummers-Pradier E, Theile G. Disclosure of new health problems and intervention planning using a geriatric assessment in a primary care setting. Croat Med J 2010;51:493-500.

37. Piccoliori G, Gerolimon E, Abholz H. Geriatric assessment in general practice using a screening instrument: is it worth the effort? Results of a South Tyrol Study. Age Ageing 2008;37:647-652.

38. Pialoux T, Goyard J, Lesourd B. Screening tools for frailty in primary health care: a systematic review. Geriatr Gerontol Int 2012;12:189-197.

39. Pijpers E, Ferreira I, Stehouwer CDA, Nieuwenhuijzen Kruseman AC. The frailty dilemma. Review of the predictive accuracy of major frailty scores. Eur J Intern Med 2012;23:118-123.

40. Metzelthin SF, Daniëls R, Van Rossum E, Cox K, Habets H, De Witte LP, et al. A nurse-led interdisciplinary primary care approach to prevent disability among community-dwelling frail older people: a large-scale process evaluation. Int J Nurs Stud 2013;50:1184-1196.

41. Fried LP, Tangen CM, Walston J, Newman AB, Hirsch C, Gottdiener J, et al. Frailty in older adults: evidence for a phenotype. J Gerontol A Biol Sci Med Sci 2001;56:M146-M157. 
42. Warnier RMJ. TRAZAG. Transmuraal Zorg Assessment Geriatrie [TRAZAG. Transmural Care Assessment Geriatrics]. Maastricht: Academic Hospital Maastricht / MUMC+; 2008.

43. Warnier RMJ, Debie T, Beusmans G. Complexe zorg voor ouderen. Beoordelingsinstrument voor de praktijkverpleegkundige [Complex care for older people. Assessment instrument for the practice nurse]. Tijdschr Prakt 2007;2:50-53.

44. Boeckxstaens P, De Graaf P. Primary care and care for older persons: position paper of the European Forum for Primary Care. Qual Prim Care 2011;19:369-389.

45. Dutch College of General Practitioners. NHG-Standpunt Toekomstvisie Huisartsenzorg. Huisartsgeneeskunde voor ouderen [Statement of the NHG, Future of General Practitioner Care. General practice medicine for older people]. Utrecht: NHG, 2007.

46. Walshe K. Understanding what works-and why-in quality improvement: the need for theory-driven evaluation. Int J Qual Health Care 2007;19:57-59.

47. Rogers EM. Diffusion of preventive innovations. Addict Behav 2002;27:989-993.

48. May C, Finch T, Mair F, Ballini L, Dowrick C, Eccles M, et al. Understanding the implementation of complex interventions in health care: the normalization process model. BMC Health Serv Res 2007;7:148.

49. Oakley A, Strange V, Bonell C, Allen E, Stephenson J, RIPPLE Study Team. Process evaluation in randomised controlled trials of complex interventions. Br Med J 2006;332:413-416.

50. Denis J, Hébert Y, Langley A, Lozeau D, Trottier L. Explaining diffusion patterns for complex health care innovations. Health Care Manage Rev 2002;27:60-73.

51. Faber MJ, Burgers JS, Westert GP. A sustainable primary care system: lessons from the Netherlands. J Ambulatory Care Manage 2012;35:174-181.

52. Hansen J, Van Greuningen M, Batenburg RS. Monitor multidisciplinaire samenwerking binnen de eerste lijn. Achtergronden en resultaten van een trend- en verdiepingsstudie [Monitor multidisciplinary cooperation within primary care. Background and results of a trend and in-depth study]. Utrecht: NIVEL, 2010.

53. Clark J. Preventive home visits to elderly people. Their effectiveness cannot be judged by randomised controlled trials. Br Med J 2001;323:708.

54. Eccles M, Grimshaw J, Campbell M, Ramsay C. Research designs for studies evaluating the effectiveness of change and improvement strategies. Qual Saf Health Care 2003;12:47-52.

55. Cook TD, Campbell DT. The causal assumptions of quasi-experimental practice: the origins of quasiexperimental practice. Synthese 1986;68:141-180.

56. Altman DG. Comparability of randomised groups. J Roy Stat Soc D-Sta 1985;34:125-136.

57. Edmonds WA, Kennedy TD. An applied reference guide to research designs. Quantitative, qualitative and mixed methods. Thousand Oaks, CA: Sage; 2013.

58. Johnson RB, Onwuegbuzie AJ. Mixed methods research: a research paradigm whose time has come. Educ Res 2004;33:14-26.

59. Moffatt S, White M, Mackintosh J, Howel D. Using quantitative and qualitative data in health services research. What happens when mixed method findings conflict? BMC Health Serv Res 2006;6:28.

60. Hawranik P, Pangman V. Recruitment of community-dwelling older adults for nursing research: a challenging process. Can J Nurs Res 2002;33:171-184.

61. Ridda I, Maclntyre CR, Lindley RI, Tan TC. Difficulties in recruiting older people in clinical trials: an examination of barriers and solutions. Vaccine 2010;28:901-906.

62. Mody L, Miller DK, McGloin JM, Freeman M, Marcantonio ER, Magaziner J, et al. Recruitment and retention of older adults in aging research. J Am Geriatr Soc 2008;56:2340-2348.

63. Rockwood K, Stadnyk K, Carver D, MacPherson KM, Beanlands HE, Powell C, et al. A clinimetric evaluation of specialized geriatric care for rural dwelling, frail older people. J Am Geriatr Soc 2000;48:1080-1085. 
64. Huss A, Stuck AE, Rubenstein LZ, Egger M, Clough-Gorr KM. Multidimensional preventive home visit programs for community-dwelling older adults: a systematic review and meta-analysis of randomized controlled trials. J Gerontol A Biol Sci Med Sci 2008;63A:298-307.

65. Medical Research Council. Developing and evaluating complex interventions: new guidance. London: MRC, 2008.

66. Egger M. Commentary: when, where, and why do preventive home visits work? $\mathrm{Br}$ Med J 2001;323:724-725.

67. Schut FT, Van den Berg B. Sustainability of comprehensive universal long-term care insurance in the Netherlands. Soc Policy Admin 2010;44:411-435.

68. Dutch Ministry of Health Welfare and Sport (VWS). Reform long-term care. The Hague: Dutch Ministry of Health, Welfare and Sport; 2014.

69. Oldham J, Brearley S, Chapman H, Coulter A, Dinwoodie M, Hay P, et al. One person, one team, one system. Report of the Independent Commission on Whole Person Care, 2014. 


\section{Valorisation}

Besides the scientific value of the studies as addressed in the inividual chapters of this dissertation, the results also have societal value. This valorisation section therefore addresses how this scientific knowledge can be transferred to and utilised in practice. The societal value will be reflected on from five different perspectives: (1) relevance of the scientific findings for practice; (2) non-scientific target groups to whom the findings are relevant; (3) translation of the findings in concrete activities and products; (4) innovativeness of these activities and products; and (5) planning of the valorisation trajectory.

\section{RELEVANCE FOR PRACTICE}

The scientific findings as presented in this dissertation have great practical relevance. Our population is ageing and global projections reveal that this will continue throughout the $21^{\text {st }}$ century and even at an accelerating pace in the coming decades. ${ }^{1}$ This has major consequences for the organisation of care for older people. The Dutch healthcare system is under pressure to meet the growing demand for healthcare and well-being services of older people. It is assumed that sustainable, efficient, and consumer-directed delivery of long-term care can only be guaranteed by reforming the current Dutch system of long-term care funding. ${ }^{2}$ Inherent to this reform is the accompanying strive to maintain independent living among older people for as long as possi- 
ble. The [G]OLD home visitation programme, as introduced in this dissertation, aimed to target two antecedents of independent living, namely health-related quality of life and disability. Nevertheless, it did not show convincing effects on these outcomes measures for various reasons as discussed in the General Discussion. The current findings will however assist in the ongoing search for the implementation of effective interventions that facilitate older people's possibility to live independently in their own home for as long as possible.

\section{TARGET GROUPS}

The work presented in this dissertation adds to current knowledge on the effectiveness and implementation of preventive home visits for community-dwelling older people. Apart from the academic community, the findings are of interest to several non-scientific target groups as well.

First of all, general practices directly benefit from the findings of this dissertation. The home visitation programme or elements of it, such as the [G]OLD comprehensive geriatric assessment, may also be relevant for other general practices outside those participating in the current studies as well. Moreover, all general practitioners (GPs) nationwide are confronted with older patients who sooner or later suffer from multiple and/or complex care problems. They warrant special attention of $\mathrm{GPs}^{3}$ and therefore GPs may continue to seek for ways to structurally identify (potentially) frail older people and to organise care for their expanding older patient population. Although the effectiveness of the [G]OLD home visitation programme is limited based on studies conducted in the south of the Netherlands, the intervention protocol or parts of it may prove effective in a different context. However, the various studies in this dissertation describe not only the effectiveness of home visits by practice nurses (PNs), but also provide detailed insight into the implementation in general practices. For example, according to GPs the systematic approach of the [G]OLD home visitation programme allowed them to obtain a comprehensive and complete picture of older people's functioning and their social network. General practices who are involved in or intent to introduce preventive home visits for their (potentially) frail older patients are advised to pay attention to advantages and drawbacks related to the implementation of the [G]OLD home visitation programme to increase the likelihood of obtaining successful outcomes in older patients.

Second, the results are relevant to other professionals who are involved in the care and/or cure network surrounding a community-dwelling older person, such as home care nurses/district nurses, physiotherapists, occupational therapists, and geriatricians. These different professionals need to align care in order to efficiently collabo- 
rate in offering the best possible care for the older person. In some instances, different professionals may already collaborate in multidisciplinary meetings. The current findings can help to understand the roles of the GP and the PN in preventive home visits and draw attention to the factors that facilitate successful collaboration between professionals.

Third, health insurers may benefit from the results of this dissertation. Health insurance companies are increasingly confronted with a transition from the current fee-for-service payments to a bundled payment system (i.e., health insurers pay a single fee to a multidisciplinary care group to cover different elements of care, for example for patients with chronic diseases). ${ }^{4}$ This also applies to care for older people. The findings may inform health insurers which elements of care provision and implementation strategies may qualify for these bundled payment contracts.

Fourth, the findings will be of interest to municipalities. As of January 2015, most of the long-term care services currently covered by the Exceptional Medical Expenses Act (abbreviated as 'AWBZ') are transferred to the new national Health Insurance Act for curative health services (abbreviated as ' $Z V W$ ') and the remaining services (related to social support and participation) into the new Social Support Act (abbreviated as 'WMO'). ${ }^{2}$ Under the latter Act, responsibilities for assigning the remaining services are delegated to municipalities. Consequently, they strive to prevent long-term care and to facilitate independent living among older people. The present dissertation provides detailed information and lessons learned from a preventive home visitation programme that could be used for this purpose. Municipalities may use the current knowledge in seeking possibilities for public health, in terms of home and community care, and primary care to combine forces in more effectively organising care for all older citizens within a municipality.

Fifth, on a national level, the results are of interest to the government. Together with other studies funded by the Netherlands Organisation for Health Research and Development ('ZonMw), as part of The National Care for the Elderly Programme, the findings assist in deciding which care models are effective and/or worth to disseminate on a larger scale. Furthermore, the studies in this dissertation draw attention to the gaps in current knowledge with respect to preventive home visits. This can be used by the government and funding agencies as background information upon which to decide about the implementation and funding of similar or alternative preventive interventions for older people in the Netherlands.

Last but not least, the findings are relevant to older people as they are the main target group of the work presented in this dissertation. Our findings showed that older people greatly appreciated the home visit. Older people wish to live independently in their own home for as long as possible. ${ }^{5}$ Based on the findings of the studies in this dissertation, they may learn what to expect from preventive home visits and thereby 
form a deliberate opinion on whether this type of preventive care is in line with their needs and preferences.

\section{ACTIVITIES AND PRODUCTS}

The concrete product resulting from the current work is the [G]OLD home visitation programme, consisting of a comprehensive geriatric assessment, a tailored care and treatment plan, multidisciplinary care management, and targeted intervention and follow-up. It offers general practices a stepwise approach for detecting and addressing health and/or well-being problems and needs among potentially frail older people ( $\geq 75$ years) at an early stage. All elements of the [G]OLD home visitation programme, as well as a general description of the development process and philosophy behind the [G]OLD home visitation programme, are described on the website of the primary healthcare organisation ' $\mathrm{IIO}^{\text {' }}$.

Process-related and implementation issues derived from the studies in this dissertation were fed back to the primary healthcare organisations who were co-responsible for the development and deploy of the [G]OLD-home visitation programme. This led for example to adaptations in the selection procedure of older people eligible to receive a home visit, adaptations to the comprehensive geriatric assessment instrument, and regular group meetings with PNs to discuss their experiences with performing the home visits and their needs for supplementary information or training. Most importantly, the primary healthcare organisation ' $\mathrm{ZIO}$ ' in the south of the Netherlands initiated negotiations with health insurers to achieve structural funding based on a bundled payment system. This was done despite the absence of convincing positive effects of the [G]OLD home visitation programme on the outcome measures healthrelated quality of life and disability, since the process evaluation did show certain promising results. This, combined with the positive reactions of GPs, PNs and older people on the [G]OLD home visitation programme, was sufficient reason for ZIO to consider it a promising approach for general practices. Also, the lack of effects on the outcomes measures may largely be attributed to implementation failure or methodological shortcomings instead of intervention failure.

\section{INNOVATIVENESS}

The [G]OLD comprehensive geriatric assessment for community-dwelling older people is unique in that it is developed by an expert panel to encompass all components of interest to a GP to obtain a complete overview of the older person's health and well- 
being. It not only concentrates on physical functioning, but also on psychological and social functioning. Furthermore, innovative is the full integration of the [G]OLD home visitation programme in primary care and the key role of the GP, supported by a PN, in coordinating care and well-being activities.

In addition, innovative is that the primary healthcare organisation ' $\mathrm{ZIO}$ ' was successful in negotiating with health insurers for a bundled payment system to structurally fund proactive care based on the [G]OLD home visitation programme for older people living in the most southern part of the Netherlands.

\section{PLANNING}

The products and activities described earlier have already been realised to a large extent. The question remains whether the [G]OLD home visitation programme is effective for community-dwelling older people, as we found no convincing evidence based on our effect evaluation. Still necessary to enhance the potential effectiveness of the [G]OLD home visitation programme on the patient level is an improvement of the system of care surrounding older people as discussed in the General Discussion. Different care and well-being services need to collaborate more extensively and more efficiently in organising the best possible care in order to maintain independent living among older people. As of January 2015, this will be stimulated by the decentralisation of long-term care for ill people and older people to municipalities. This may have consequences for the role of the general practice in performing the home visits of the [G]OLD home visitation programme. By working together with local health services and social care services at neighbourhood level, new alliances between public health and primary care may arise. Monitoring is needed to find out how the [G]OLD home visitation programme can be adapted to fit to these developments without discarding its active ingredients. 


\section{REFERENCES}

1. Lutz W, Sanderson W, Scherbov S. The coming acceleration of global population ageing. Nature 2008;451:716-719.

2. Schut FT, Van den Berg B. Sustainability of comprehensive universal long-term care insurance in the Netherlands. Soc Policy Admin 2010;44:411-435.

3. Dutch College of General Practitioners. NHG-Standpunt Toekomstvisie Huisartsenzorg. Huisartsgeneeskunde voor ouderen [Statement of the NHG, Future of General Practitioner Care. General practice medicine for older people]. Utrecht: NHG, 2007.

4. De Bakker DH, Struijs JN, Baan CA, Raams J, De Wildt J, Vrijhoef HJM, et al. Early results from adoption of bundled payment for diabetes care in the Netherlands show improvement in care coordination. Health Aff (Millwood) 2012;31:426-433.

5. De Blok C, Meijboom B, Luijkx K, Schols J. Demand-based provision of housing, welfare and care services to elderly clients: from policy to daily practice trough operations management. Health Care Analysis 2009;17:86-84.

6. ZIO Zorg In Ontwikkeling. Goede zorg voor ouderen: keten complexe zorg. Maastricht: ZIO; 2014 [cited 2014 November 26th]. Available from: http://www.zio.nl/zorgprogrammas/ouderenzorg/algemeneinformatie/ 


\section{Summary}

Population ageing poses challenges for healthcare systems worldwide. Due to declining birth rates and increased longevity, there is a rise in the number of older people who live into old age. Although many people will reach a high age in relatively good health, a growing number of older people faces complex health and/or well-being problems at a certain point in their lives. As a result, they experience restrictions in the performance of meaningful daily activities which may threaten their ability to live independently. These older people are considered vulnerable or 'frail'. It has been recognised that potentially frail older people should be identified at an early stage to prevent unnecessary neediness and maintain or improve independent functioning. General practices within the primary care setting have the potential to deliver this type of proactive care in line with older people's health and well-being needs.

This dissertation reports on a comprehensive evaluation regarding the feasibility and effectiveness of the [G]OLD preventive home visitation programme for potentially frail community-dwelling older people (aged $\geq 75$ years) in general practices in the south of the Netherlands. The intervention protocol of the [G]OLD home visitation programme applies a stepwise approach, starting with the Practice Nurse (PN) planning an appointment for the home visit (step 1) and preparing the visit by shortly reviewing the older person's medical history (step 2). The PN then visits the older person at home. Here, he/she performs a comprehensive geriatric assessment of the older person's health and well-being using the so-called [G]OLD-instrument (step 3). Results of the assessment are discussed with the General Practitioner (GP) (step 4), as well as 
with the older person (step 5). In case problems or risk situations require attention, a care and treatment plan is formulated in accordance with the older person's needs and wishes (step 6). Subsequently, the PN executes the care and treatment plan, for example by referring the older person to individually appropriate professionals (e.g., physiotherapist, occupational therapist) (step 7). Finally, the PN coordinates care, monitors progress, and arranges follow-up appointments (e.g., monthly contact or annual re-assessment with the [G]OLD-instrument) (step 8).

Chapter 1 presents the rationale for conducting the work described in this dissertation. It elaborates on the challenges imposed by population ageing in terms of frailty and disability, presents the changes needed in our healthcare system to adequately care for older people, explores the appropriateness of the primary care setting for improving care for older people, summarises current evidence regarding the effectiveness of preventive home visitation programmes, and identifies gaps in the literature.

Chapter 2 describes the process of the development of the [G]OLD home visitation programme. A pilot study was conducted to evaluate the initial feasibility of preventive home visits by PNs, practical usefulness of the [G]OLD comprehensive geriatric assessment instrument, and to what extent the different steps of the [G]OLD home visitation programme were performed according to the protocol. Twenty-one general practices participated and 240 community-dwelling older people (aged $\geq 75$ years) were visited at home by the PN. Using a mixed-model design, this study revealed that the [G]OLD home visitation programme was feasible in daily practice according the GPs and PNs. Apart from some minor adjustments needed, the [G]OLD comprehensive geriatric assessment instrument was considered useful by both GPs and PNs. Finally, protocol deviations (e.g., skipping items of the [G]OLD-instrument, inaccurate or inadequate reporting of the care and treatment plan by the PN) offered insight into the necessary improvements for optimal performance of the [G]OLD home visitation programme beyond the pilot phase.

After the pilot phase, the [G]OLD home visitation programme was introduced to other general practices in the regions 'Maastricht-Heuvelland' and 'Parkstad' as well. To thoroughly investigate the implementation of the [G]OLD home visitation programme in general practices, a comprehensive process evaluation plan was written, entailing a mixed-method study among GPs, PNs, and older people from the 13 general practices (out of 24 general practices who participated in this evaluation research) who implemented the [G]OLD home visitation programme (intervention practices). This plan is presented in Chapter 3. In addition, arguments for prospectively developing a process evaluation plan and for conducting a process evaluation alongside a longitudinal trial are given.

The findings of the process evaluation are addressed in Chapter 4. Both GPs and PNs evaluated the [G]OLD home visitation programme positively, because it resulted in 
more attention for older people within their general practice, closer collaboration between the GP and PN, and a comprehensive picture of older people's functioning and social network. The older people themselves were satisfied with the home visit, as it offered them the ability to express their daily concerns, such as impairments associated with ageing. Overall, the [G]OLD home visitation programme did not have any major shortcomings in itself, but suffered from implementation failure and the uncertain commitment of general practices towards sustained redesign in care delivery from reactive, disease-oriented care towards proactive, patient-oriented care. Threats to complete delivery of the intervention were related to few new health and/or wellbeing problems being detected among older people, which caused certain elements of the intervention protocol to be non-applicable. Furthermore, PNs perceived a lack of time, which could explain why various elements of the [G]OLD home visitation programme were not delivered as planned. The time investment and corresponding available financial reimbursement by health insurers were considered disproportionate compared to the benefits in terms of detected problems.

Both the pilot study as well as the process evaluation showed that PNs often did not formulate care and treatment plans or they formulated them inappropriately (e.g., incomplete, no concrete goals). Therefore, Chapter 5 describes an additional crosssectional, mixed-model study to investigate PNs' actions and views regarding (1) the formulation of a care and treatment plan for older people and (2) the adherence to the care and treatment plan and monitoring during follow-up of older people in whom health and/or well-being problems had been detected. The findings showed that only $27.8 \%$ of the problems identified through the [G]OLD comprehensive geriatric assessment were included in a care and treatment plan by the PN. According to the PN, the main reason for not including an identified problem in the plan was because it was an existing problem and/or action had already been undertaken. Both patient-related factors (e.g., the older person's wishes) and problem-specific factors (e.g., perceived seriousness of the problem) influenced the formulation of care plans. If problems were registered in the care and treatment plan, PNs mostly adhered to it as shown by the finding that $62.9 \%$ of the registered problems were dealt with according to PNs.

Chapter 6 presents the design of the longitudinal, quasi-experimental study to evaluate effectiveness of the [G]OLD home visitation programme on older people's health-related quality of life and disability (primary outcome measures), as well as attitude towards ageing (secondary outcome measure). Twenty-four general practices participated of which 13 general practices redesigned their care delivery to older people and implemented the [G]OLD home visitation programme (intervention practices), whereas 11 general practices provided usual care to older people (control practices). Community-dwelling older people aged $\geq 75$ years were eligible to participate. In total, 4,588 eligible older people were approached for participation and 1,737 older people 
(37.9\%) consented to participate. The outcome measures were measured by means of a self-reported questionnaire at baseline (prior to the home visit) and after 6,12 , and 18-months follow-up.

The results of the longitudinal, quasi-experimental study to examine the effectiveness of the [G]OLD home visitation programme are described in Chapter 7. We found no convincing effects of the [G]OLD home visitation programme compared to usual care. Mixed regression models only showed a marginally significant effect of the [G]OLD home visitation programme in favour of the intervention group on role limitations due to physical problems at 6 months and 18 months follow-up compared to the control group. No statistically significant effects in favour of the intervention group were found on all other outcome measures and time points.

A solution proposed by GPs and PNs to reduce the time investment of the [G]OLD home visitation programme was to target the intervention to those older people who most likely benefit from it. Therefore, Chapter 8 reports on a cross-sectional study among 45 general practices who implemented the [G]OLD home visitation programme to determine which community-dwelling older people (aged $\geq 75$ years) benefit most from the [G]OLD comprehensive geriatric assessment during a home visit in terms of detected health and/or well-being problems. Thirteen general practices who participated in the quasi-experimental trial randomly selected community-dwelling older people, which resulted in the inclusion of 562 older people in this study. The remaining 32 general practices chose a different selection procedure by purposefully selecting older people who fulfilled one of the following criteria according to the GP: last visit to general practice > 6 months ago; partner or child(ren) deceased within past 12 months; cognitive or psychosocial functioning unknown to GP; $\geq 2$ chronic conditions; uses $\geq 5$ medications and/or living alone. The findings revealed that even though observed differences in detected problems between the two selection procedures were relatively small, more problems were detected in purposefully selected older people than in randomly selected older people. Among the purposeful selection criteria, most problems were detected in older people with $\geq 2$ chronic conditions and those using $\geq 5$ medications. Furthermore, more problems were detected among women, higher age groups, those living alone and those less educated. This suggests that the [G]OLD home visitation programme should preferably be targeted to purposefully selected older people.

Finally, Chapter 9 summarises and critically reflects on the main findings, elaborates on methodological considerations of the work presented in this dissertation, and discusses the implications for further research and for practice. In conclusion, the routine dissemination and implementation of the [G]OLD home visitation programme in general practices in its current form is doubtful. However, considering the ageing of our population and concomitant healthcare reforms planned for the near future, the 
need for general practices to redesign their care delivery to older people will persist. The information presented in this dissertation warrants further consideration before undertaking any comparable or adjusted home visitation programme for communitydwelling older people ( $\geq 75$ years) within general practices or other multidisciplinary care service teams. 



\section{Samenvatting}

De vergrijzing van de populatie leidt wereldwijd tot uitdagingen voor gezondheidszorgsystemen. Door afnemende geboortecijfers en een toegenomen levensverwachting vindt er een stijging plaats in het aantal ouderen die een hoge leeftijd bereiken. Hoewel veel mensen een hoge leeftijd zullen bereiken terwijl ze een relatief goede gezondheid hebben, is er een toenemend aantal ouderen dat op een bepaald moment in hun leven te maken krijgt met complexe zorg- en/of welzijnsproblemen. Als gevolg hiervan ervaren ze beperkingen in het uitvoeren van betekenisvolle dagelijkse activiteiten wat een bedreiging vormt voor hun mogelijkheid om zelfstandig te blijven wonen. Deze ouderen worden gezien als kwetsbaar of fragiel. Ouderen in een mogelijk kwetsbare positie moeten vroegtijdig worden geïdentificeerd om onnodige hulpbehoevendheid te voorkomen en zelfstandig functioneren te handhaven of bevorderen. Huisartsenpraktijken in de eerstelijnszorg hebben de mogelijkheid om deze vorm van proactieve zorg te leveren in overeenstemming met de gezondheids- en welzijnsbehoeften van ouderen.

Dit proefschrift beschrijft een uitgebreide evaluatie met betrekking tot de praktische uitvoerbaarheid en effectiviteit van het [G]OUD project ('Gezond Oud in Limburg'), bestaande uit preventieve huisbezoeken voor zelfstandig wonende ouderen (75 jaar en ouder) in een mogelijk kwetsbare positie, uitgevoerd in huisartsenpraktijken in het zuiden van Nederland. Het interventieprotocol van het [G]OUD-project maakt gebruik van een stapgewijze benadering, waarbij gestart wordt met de $\mathrm{POH}$ (PraktijkOndersteuner Huisarts) die een afspraak plant voor het huisbezoek (stap 1) en 
het huisbezoek voorbereidt door kort een blik te werpen op de medische achtergrond van de oudere (stap 2). De POH bezoekt de oudere vervolgens thuis. Daar voert hij/zij een uitgebreide geriatrische screening uit van de gezondheid en het welzijn van de oudere met behulp van het zogenaamde [G]OUD-instrument (stap 3). Resultaten van deze screening worden besproken met de huisarts (stap 4), evenals met de oudere zelf (stap 5). Wanneer er problemen of risicosituaties zijn gesignaleerd die aandacht behoeven, wordt een zorgbehandelplan opgesteld dat aansluit op de wensen en behoeften van de oudere (stap 6). Vervolgens voert de $\mathrm{POH}$ het zorgbehandelplan uit, bijvoorbeeld door de oudere door te verwijzen naar voor de oudere geschikte professionals (bijv. fysiotherapeut, ergotherapeut) (stap 7). Tot slot coördineert de $\mathrm{POH}$ de zorg, monitort vooruitgang en regelt vervolgafspraken (bijv. maandelijks contact of jaarlijks herhalen van de screening met het [G]OUD-instrument) (stap 8).

Hoofdstuk 1 presenteert de achtergrond voor het uitvoeren van de studies zoals beschreven in dit proefschrift. Het gaat uitgebreid in op de uitdagingen die gepaard gaan met de vergrijzing van de populatie in termen van kwetsbaarheid en verminderde zelfredzaamheid, presenteert de veranderingen die nodig zijn in ons gezondheidszorgsysteem om adequaat te zorgen voor ouderen, gaat in op de geschiktheid van de eerstelijnssetting voor het verbeteren van ouderenzorg, geeft een samenvatting van huidig bewijs met betrekking tot de effectiviteit van preventieve huisbezoeken en geeft aan wat ontbreekt in de literatuur op dit gebied.

Hoofdstuk 2 beschrijft van de ontwikkeling van het [G]OUD-project. Een pilot studie werd uitgevoerd voor het evalueren van de praktische uitvoerbaarheid van preventieve huisbezoeken door $\mathrm{POH}$-ers, praktische bruikbaarheid van het [G]OUDinstrument en de mate waarin de verschillende stappen van het [G]OUD-project werden uitgevoerd volgens het protocol. Eénentwintig huisartsenpraktijken namen deel en 240 zelfstandig wonende ouderen (75 jaar en ouder) werden thuis bezocht door de $\mathrm{POH}$. Gebruik makende van een 'mixed-method' onderzoeksopzet, liet deze studie zien dat het [G]OUD-project praktisch uitvoerbaar was in de dagelijkse huisartsenpraktijk volgens huisartsen en $\mathrm{POH}$-ers. Behalve enkele kleine aanpassingen die nodig waren, was het [G]OUD-instrument nuttig volgens zowel huisartsen als $\mathrm{POH}$ ers. Tot slot gaven de afwijken van het protocol (bijv. weglaten van vragen in het [G]OUD-instrument, onzorgvuldig of onvolledig invullen van het zorgbehandelplan door de $\mathrm{POH}$ ) inzicht in de noodzakelijke verbeteringen om het [G]OUD-project optimaal te laten functioneren na de pilot fase.

$\mathrm{Na}$ de pilot fase werd het [G]OUD-project ook geïntroduceerd in andere huisartsenpraktijken in de regio's Maastricht-Heuvelland en Parkstand. Om de implementatie van het [G]OUD-project in huisartsenpraktijken grondig te onderzoeken, werd een uitgebreid procesevaluatieplan geschreven bestaande uit een 'mixed-method' studie onder huisartsen, $\mathrm{POH}$-ers en ouderen van de 13 huisartsenpraktijken (van de in 
totaal 24 deelnemende huisartsenpraktijken die deelnamen aan het evaluatieonderzoek) die het [G]OUD-project geïmplementeerd hadden (interventiepraktijken). Dit plan wordt gepresenteerd in Hoofdstuk 3. Bovendien worden hier argumenten geven voor het vooraf ontwikkelen van een procesevaluatieplan en voor het uitvoeren van een procesevaluatie parallel aan een longitudinale studie naar de effecten van de interventie.

De bevindingen van de procesevaluatie komen aan bod in Hoofdstuk 4. Zowel huisartsen als $\mathrm{POH}$-ers evalueerden het [G]OUD-project positief, omdat het heeft geresulteerd in meer aandacht voor ouderen binnen de huisartsenpraktijk, nauwere samenwerkingen tussen de huisarts en $\mathrm{POH}$, en een compleet beeld van het functioneren en het sociale netwerk van ouderen. De ouderen zelf waren tevreden over het huisbezoek, want het gaf hun de gelegenheid om hun dagelijkse bezorgdheden te uiten, zoals beperkingen door het ouder worden. Samenvattend bleek het [G]OUD-project inhoudelijk geen grote tekortkomingen te hebben, maar het kreeg te maken met een gebrekkige implementatie en huisartsenpraktijken die zich niet volledig committeerden aan het definitief herorganiseren van de zorg van reactieve en ziektegeoriënteerde zorg naar proactieve en patiëntgeoriënteerde zorg. Bedreigingen voor het volledig uitvoeren van de interventie waren gerelateerd aan het feit dat er weinig nieuwe gezondheids- en/of welzijnsproblemen waren opgespoord bij ouderen, waardoor bepaalde onderdelen van het interventieprotocol niet van toepassing waren. Bovendien ervaarden $\mathrm{POH}$-ers een een gebrek aan tijd, wat kan verklaren waarom verscheidene elementen van het [G]OUD-project niet werden uitgevoerd volgens plan. De tijdsinvestering en daarmee gepaard gaande beschikbare financiële vergoedingen van zorgverzekeraars werden gezien als disproportioneel vergeleken met de voordelen in termen van opgespoorde problemen.

Zowel de pilot studie als ook de procesevaluatie toonden aan dat $\mathrm{POH}$-ers vaak geen zorgbehandelplannen formuleerden of ze formuleerden ze niet naar behoren (bijv. onvolledig, geen concrete doelen). Daarom wordt in Hoofdstuk 5 een aanvullend dwarsdoorsnede-onderzoek beschreven waarbij gebruik wordt gemaakt van een 'mixed-model' onderzoeksopzet om de gedragingen en standpunten van $\mathrm{POH}$-ers te onderzoeken met betrekking tot (1) het formuleren van een zorgbehandelplan voor ouderen en (2) het zich houden aan het zorgbehandelplan en het monitoren van ouderen waarbij zorg- en/of welzijnsproblemen werden opgespoord. De bevindingen laten zien dat slechts $27,8 \%$ van de problemen die werden opgespoord met het [G]OUD-instrument werden opgenomen in een zorgbehandelplan door de $\mathrm{POH}$. Volgens de $\mathrm{POH}$ was de belangrijkste reden voor het niet opnemen van een opgespoord probleem in het zorgbehandelplan dat het een bestaand probleem was en/of een probleem waarvoor al eerder actie was ondernomen. Zowel patiëntgerelateerde factoren (bijv. de wensen van de oudere) en probleemspecifieke factoren 
(bijv. de ervaren ernst van het probleem) hadden invloed op het opstellen van zorgbehandelplannen. Wanneer problemen wel werden opgenomen in het zorgbehandelplan dan volgden $\mathrm{POH}$-ers die meestal ook op, zoals blijkt uit het resultaat dat $62,9 \%$ van de geregistreerde problemen waren opgepakt volgens de $\mathrm{POH}$-ers.

Hoofdstuk 6 presenteert de onderzoeksopzet van een longitudinale, quasiexperimentele studie voor het evalueren van de effectiviteit van het [G]OUD-project op de gezondheidsgerelateerde kwaliteit van leven en zelfredzaamheid van ouderen (primaire uitkomstmaten), als ook de houding die ouderen hebben ten opzichte van ouder worden (secundaire uitkomstmaat). Vierentwintig huisartsenpraktijken deden mee waarvan 13 huisartsenpraktijken hun zorg voor ouderen herorganiseerden en daarvoor het [G]OUD-project implementeerden (interventiepraktijken), terwijl 11 huisartsenpraktijken de gebruikelijke zorg leverden aan ouderen (controlepraktijken). Zelfstandig wonende ouderen van 75 jaar en ouder kwamen in aanmerking voor deelname. In totaal werden 4.588 ouderen die in aanmerking kwamen voor deelname benaderd en 1.737 ouderen (37,9\%) gaven toestemming voor deelname. De uitkomstmaten werden gemeten door middel van een zelf in te vullen vragenlijst die naar ouderen werd verstuurd tijdens de start (voorafgaande aan het huisbezoek) en na 6 maanden, 12 maanden en 18 maanden.

De resultaten van de longitudinale, quasi-experimentele studie voor het evalueren van de effectiviteit van het [G]OUD-project zijn beschreven in Hoofdstuk 7. We vonden géén overtuigende effecten van het [G]OUD-project vergeleken met gebruikelijke zorg. Mixed regressiemodellen lieten alleen een klein significant effect zien van het [G]OUDproject ten gunste van de interventiegroep op rolbeperkingen door lichamelijke problemen na 6 maanden en na 18 maanden vergeleken met de controlegroep. $\mathrm{Er}$ werden geen statistisch significante effecten gevonden ten gunste van de interventiegroep voor alle andere uitkomstmaten en vervolgmetingen.

Een oplossing die werd voorgesteld door huisartsen en $\mathrm{POH}$-ers voor het verminderen van de tijdsinvestering van het [G]OUD-project was om de interventie te richten op die ouderen die er naar alle waarschijnlijkheid het meeste van zullen profiteren. Hoofdstuk 8 beschrijft daarom ook een dwarsdoorsnede-onderzoek onder 45 huisartsenpraktijken die het [G]OUD-project hebben geïmplementeerd om te bepalen welke zelfstandig wonende ouderen (75 jaar en ouder) het meeste profiteren van de [G]OUD-screening tijdens een huisbezoek in termen van het aantal opgespoorde zorgen/of welzijnsproblemen. Dertien huisartsenpraktijken die deelnemen aan de quasiexperimentele studie hadden willekeurig zelfstandig wonende ouderen geselecteerd en dit leidde tot de inclusie van 562 ouderen in deze deelstudie. De overige 32 huisartsenpraktijken kozen voor een andere selectieprocedure, namelijk het doelgericht selecteren van ouderen die voldeden aan een van de volgende criteria volgens de huisarts: laatste bezoek aan de huisartsenpraktijk was langer dan 6 maanden 
geleden; partner of kind(eren) overleden in de afgelopen 12 maanden; cognitief of psychosociaal functioneren onbekend bij de huisarts; $\geq 2$ chronische ziekten; degenen die $\geq 5$ medicamenten gebruiken; alleenstaanden. Uit de bevindingen bleek dat ondanks dat de geobserveerde verschillen in opgespoorde problemen tussen de 2 selectieprocedures relatief klein waren, er meer problemen werden opgespoord bij de doelgericht geselecteerde ouderen dan bij de willekeurig geselecteerde ouderen. Wat betreft de doelgerichte selectiecriteria werden de meeste problemen opgespoord bij ouderen met $\geq 2$ chronische ziekten en degenen die $\geq 5$ medicamenten gebruiken. Bovendien werden meer problemen opgespoord bij vrouwen, bij de hogere leeftijdsgroepen, bij alleenstaanden en bij de lager opgeleiden. Dit suggereert dat het [G]OUD-project bij voorkeur zich zou moeten richten op doelgericht geselecteerde ouderen.

Tot slot vat Hoofdstuk 9 alles samen en reflecteert kritisch op de belangrijkste bevindingen, gaat in op de methodologische overwegingen van de studies in dit proefschrift en bediscussieert de implicaties voor verder onderzoek en voor de praktijk. Samenvattend kan gesteld worden dat het op grote schaal verspreiden en implementeren bij huisartsenpraktijken van het [G]OUD-project in de huidige vorm discutabel is. Echter, gezien de vergrijzing van de populatie en daarmee gepaard gaande hervormingen in de gezondheidszorg in de nabije toekomst, zal de behoefte van huisartsenpraktijken voor het herorganiseren van hun zorg voor ouderen blijven gelden. De informatie gepresenteerd in dit proefschrift vereist zorgvuldige aandacht alvorens vergelijkbare of aangepaste projecten worden ondernomen die bestaan uit huisbezoeken voor zelfstandig wonende ouderen (75 jaar en ouder) binnen de huisartsenpraktijk of andere multidisciplinaire zorgteams. 



\section{Dankwoord}

Wat is de tijd voorbij gevlogen! Met trots kijk ik terug op de afgelopen jaren waarin ik met veel plezier aan mijn onderzoek heb gewerkt. Daarbij heb ik het geluk gehad te mogen samenwerken met mensen die mij hebben geïnspireerd, ondersteund en gemotiveerd in de uitvoering van dit onderzoek. Deze laatste woorden spendeer ik dan ook graag aan iedereen die op welke manier dan ook een bijdrage heeft geleverd aan de totstandkoming van dit proefschrift. Mijn dank daarvoor! Een aantal personen wil ik hier graag persoonlijk bedanken.

Allereerst een speciaal woord van dank aan mijn promotieteam. Bedankt voor de fijne samenwerking de afgelopen jaren waarin ik veel van jullie heb geleerd. Ik waardeer het vertrouwen en de vrijheid die jullie mij gaven bij de uitvoering van mijn onderzoek. Daarnaast hebben jullie mij altijd ondersteund om het maximale uit mezelf en het onderzoek te halen. Bert, als promotor heb jij altijd de grote lijnen bewaakt. Door kritische vragen te stellen vanuit net weer een ander standpunt werd ik steeds geprikkeld om anders naar mijn onderzoek te kijken. Ik dank je voor de kansen die je mij bood en jouw waardevolle bijdrage. Maria, als co-promotor en inmiddels ook promotor heb je altijd de verbinding tussen onderzoek en praktijk bewaakt. Jouw expertise op dit gebied was voor mij onmisbaar. Ondanks drukte was je altijd nauw betrokken en jouw gedrevenheid en enthousiasme werkten aanstekelijk. Inge, ik had me geen betere co-promotor kunnen wensen! Zowel inhoudelijk als op persoonlijk vlak heb je een 
belangrijke bijdrage geleverd aan mijn promotietraject. Ik heb veel geleerd van jouw kritische blik en doortastendheid. Bedankt voor alles!

Dit onderzoek was niet mogelijk geweest zonder de medewerking van enkele huisartsenpraktijken en vele ouderen. Mijn dank gaat dan ook uit naar alle deelnemende ouderen die telkens weer bereid waren een vragenlijst in te vullen. Daarnaast dank ik alle huisartsenpraktijken in de regio's Maastricht-Heuvelland, Oostelijk Zuid-Limburg en Midden-Limburg voor hun medewerking aan het onderzoek. In het bijzonder bedank ik de POH-ers uit de interventiegroep: Shirley, Denise, Marinka, Rob, Tiny, Petra, Audrey, Truus, Kim, Gaby, Suzanne, Brenda en Felisa. Het was prettig om met jullie samen te werken en ik dank jullie voor jullie continue inzet om het onderzoek te laten slagen.

De huisartsenorganisaties ZIO, HOZL en Meditta dank ik voor hun medewerking in de totstandkoming van het onderzoek. Vanuit ZIO, voorheen RHZ Heuvelland, heb ik in de beginfase mijn werkzaamheden verricht. Marianne, Ingeborg, Maud en Jolanda, jullie dragen [G]OUD een warm hart toe. Het was fijn om samen met jullie het project vorm te geven en uit te voeren.

Ook dank ik de co-auteurs van de artikelen in dit proefschrift. Ingeborg, Maud en Yvonne, bedankt voor het kritisch becommentariëren van mijn stukken. Math, bedankt voor de prettige samenwerking bij de analyse van de effectstudie. Het geduld en gemak waarmee jij ingewikkelde statistische analyses toch begrijpelijk kunt uitleggen, is bewonderenswaardig.

Dat bij een ouderenzorgproject heel veel papierwerk komt kijken, weten Susanne en Marion inmiddels wel! Susanne, jij hebt incidenteel bijgesprongen als er weer eens een piek was qua werving of dataverwerking. Bedankt daarvoor! Marion, als vaste onderzoeksassistente voor [G]OUD hebben we veel samengewerkt. Of het nou ging om werving, dataverzameling of data-invoer, jij hebt zo'n beetje overal aan meegewerkt. Het was fijn dat ik op jou kon vertrouwen dat alles wel in goede banen werd geleid. Bedankt voor de prettige samenwerking!

Ik bedank alle collega's van de vakgroep Huisartsgeneeskunde voor de fijne tijd die ik op de vakgroep heb gehad en de gezellige praatjes op de gang. In het bijzonder bedank ik Viola, Janaica, Martine, Petra, Sil, Esther en Tineke. Dat ik met veel plezier naar mijn werk ging, kwam mede door jullie! De vele lunchwandelingen en praatjes waarin we lief en leed deelden zal ik niet gauw vergeten. Ik ben dan ook blij dat we elkaar zo nu 
en dan nog eens zien of spreken. Viola, met jou heb ik de langste tijd een kamer gedeeld en ik had me geen fijner kamergenootje kunnen wensen. Wat hebben we een gezellige maar ook productieve tijd gehad! Doordat we zowel wat betreft ons promotietraject als ook ons privéleven nagenoeg in een gelijke fase zaten, hebben we een speciale band opgebouwd. Bedankt voor alles! Verder natuurlijk ook dank aan Ine, Kim, Judith, Ellen, Jim, Marga en Jos voor jullie hulp de afgelopen jaren bij veelal praktische zaken of ICT-problemen.

Dankbaar ben ik voor de vele gezellige en inspirerende avonden van de Journal Club Maastricht. Het was fijn om samen met jullie ook na werktijd te discussiëren over de zin en onzin van wetenschap onder het genot van een heerlijke maaltijd. Ik heb ervan genoten!

Vrienden en familie wil ik graag bedanken voor hun steun en interesse. Lieke en Anne, wat hebben we een fijne vriendschap! Dat we na onze studie ook nog eens alle drie een promotietraject succesvol hebben afgerond, is toch wel speciaal. Ik ben trots op jullie! Lieke, wat fijn dat jij als paranimf vandaag naast me staat. Mam en pap, bedankt voor jullie betrokkenheid en onvoorwaardelijke steun bij alles wat ik doe. Heel fijn dat ik voor het afronden van mijn proefschrift nog eens bij jullie op zolder op mijn oude studeerplek mocht komen zitten. Ik weet dat ik altijd bij jullie terecht kan. Oma, het zal misschien altijd vaag blijven wat voor een werk ik deed, maar wat maakt het eigenlijk ook uit. Ik ben blij om zo'n trotse oma te hebben. Jammer dat opa dit niet meer meemaakt, hij zou ook ontzettend trots zijn geweest. Deborah, jouw reactie op mijn Facebook bericht naar aanleiding van een publicatie van één van mijn artikelen zal ik nooit vergeten: "Geen idee wat het betekent, maar het zal vast goed zijn." Ik ben je heel dankbaar voor het maken van de mooie kaft voor dit proefschrift. Misschien heb je hierdoor een beetje meer zicht gekregen op wat ik eigenlijk al die jaren gedaan heb. Heel speciaal dat je ook nog eens als paranimf naast me staat! Mijn schoonouders, Ans en Edy, bedankt voor jullie interesse in mijn werk en het gezellige samenzijn.

Lieve Rick en Cas, de laatste woorden zijn voor jullie. Door jullie weet ik waar het in het leven om draait. Er is met geen pen te beschrijven hoeveel jullie voor mij betekenen. Laten we nu lekker samen genieten van al het moois dat de toekomst ons nog gaat brengen.

\section{Mandy}

Elsloo, december 2014 



\section{Curriculum Vitae}

Mandy Mathilda Nicolaas Stijnen was born on August 14th, 1986 in Geleen, the Netherlands. In 2004, she completed secondary school (higher secondary education, 'VWO') at Groenewald in Stein. Hereafter, she studied Health Sciences at Maastricht University, with a special focus on Health Education and Promotion (major) and Movement Sciences (minor). She obtained her bachelor degree 'with distinction' in 2007. Mandy continued her studies at Maastricht University and was enrolled in the Health Sciences Research Master, with a specialisation in Social Sciences. She graduated 'cum laude' in 2009. Upon graduation, she started working as a PhD-student at department of Family Medicine which resulted in this dissertation. As of May 2014, Mandy is working as a researcher at the Veiligheidsregio Limburg-Noord in Venlo. 



\section{Publication list}

\section{Publications from this dissertation}

Stijnen MMN, Jansen MWJ, Duimel-Peeters, IGP, Vrijhoef HJM. Nurse-led home visitation programme to improve health-related quality of life and reduce disability among potentially frail community-dwelling older people in general practice: a theorybased process evaluation. BMC Fam Pract 2014;15:173.

Stijnen MMN, Duimel-Peeters IGP, Vrijhoef HJM, Jansen MWJ. Process evaluation plan of a patient-centred home visitation program for potentially frail community-dwelling older people in general practice. Eur J Pers Cent Healthc 2014;2:179-189.

Stijnen MMN, Vrijhoef HJM, Duimel-Peeters IGP, Jansen MWJ. Formulation of and adherence to a care plan for potentially frail community-dwelling older people by practice nurses in the Netherlands. J Nurs Educ Pract 2014;4:34-43.

Stijnen MMN, Van Hoof MS, Wijnands-Hoekstra IYM, Guldemond-Hecker Y, DuimelPeeters IGP, Vrijhoef HJM, et al. Detected health and well-being problems following comprehensive geriatric assessment during a home visit among community-dwelling older people: who benefits most? Fam Pract 2014;31:333-340. 


\section{PUBLICATION LIST}

Stijnen MMN, Duimel-Peeters IGP, Jansen MWJ, Vrijhoef HJM. Early detection of health problems in potentially frail community-dwelling older people by general practices - project [G]OLD: design of a longitudinal, quasi-experimental study. BMC Geriatr 2013;13:7.

Stijnen MMN, Jansen MWJ, Vrijhoef HJM, Duimel-Peeters IGP. Development of a home visitation programme for the early detection of health problems in potentially frail community-dwelling older people by general practices. Eur J Ageing 2013;10:49-60.

\section{Additional publications}

Dijker AJM, Nelissen RMA, Stijnen MMN. Framing posthumous organ donation in terms of reciprocity: what are the emotional consequences? Basic App Soc Psych 2013;25:256-264.

Stijnen MMN, Dijker AJM. Reciprocity and need in posthumous organ donation: the mediating role of moral emotions. Soc Psychol Person Sci 2011;2:387-394.

Dijker AJM, Stijnen MMN. Orgaandonatie: altruïsme, coöperatie of beide? Ned Tijdschr Geneeskd 2009;153:592-594. 

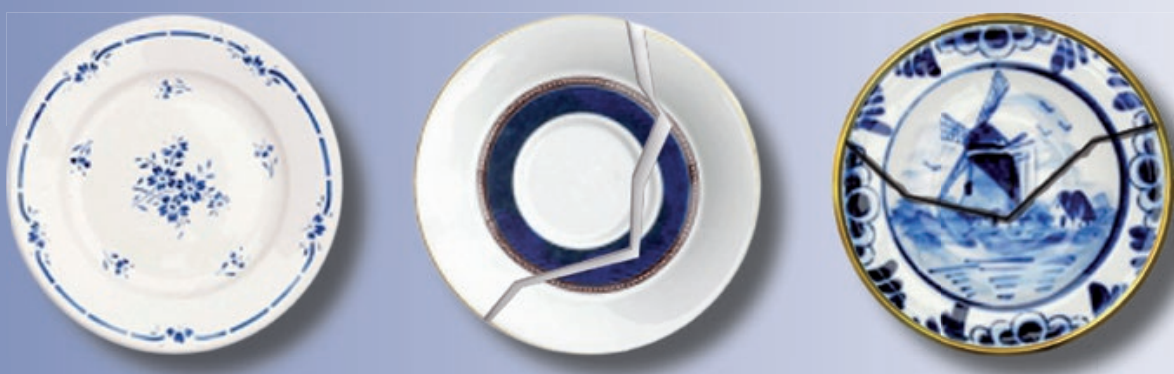